
Propositions.

1. Virus-induced gene silencing is the most powerful technology to induce mutant phenotypes and simultaneously generate mutation-free offspring. (this thesis)

2. Reverse breeding can only be methodically and efficiently applied in a commercial set-up through partial decrease of meiotic recombination frequencies.

(this thesis)

3. The scientific and political community should consciously, collectively and rationally work towards meeting the deliverables dates irremediably and currently imposed by climate change.

4. Exploiting the full potential of controlling meiotic recombination at will in the $\mathrm{EU}$ in the near future is only possible after the development and implementation of transient, non-GMO silencing methods for plant breeding.

5. Only those that are willing to accept a nomad lifestyle can truly pursue a career in academia.

6. The Deutsche-Bahn is a disgrace to German efficiency.

Propositions belonging to this thesis entitled "Control of plant meiosis using virusinduced gene silencing (VIGS)"

Vanesa Calvo Baltanás.

Wageningen, 15 $5^{\text {th }}$, February, 2019. 


\section{Control of plant meiosis using virus- induced gene silencing (VIGS)}

Vanesa Calvo-Baltanás 


\section{Thesis committee}

\section{Promotors}

Prof. Dr J.H.S.G.M de Jong

Personal chair at the Laboratory of Genetics

Wageningen University \& Research

Prof. Dr B.J Zwaan

Professor of Genetics

Wageningen University \& Research

\section{Co-promotors}

Dr T.G Wijnker

Post-doctoral researcher at the Laboratory of Genetics

Wageningen University \& Research

Prof. Dr A. Schnittger

Professor at the Department of Developmental BiologyHamburg University, Germany

\section{Other members}

Prof. Dr M.M. van Oers, Wageningen University \& Research

Prof. Dr Y. Bai, Wageningen University \& Research

Prof. Dr N. de Storme, KU Leuven, Belgium

Dr P.F. Fransz, University of Amsterdam

This research was conducted under the auspices of the Graduate School Experimental Plant Sciences (EPS). 


\title{
Control of plant meiosis using virus- induced gene silencing (VIGS)
}

\author{
Vanesa Calvo-Baltanás
}

\section{Thesis}

submitted in fulfilment of the requirements for the degree of doctor

at Wageningen University

by the authority of the Rector Magnificus,

Prof. Dr A.P.J. Mol,

in the presence of the

Thesis Committee appointed by the Academic Board

to be defended in public

on Friday 15 February 2019

at 4 p.m. in the Aula. 
Vanesa Calvo-Baltanás

Control of plant meiosis using virus-induced gene silencing (VIGS), 164 pages.

$\mathrm{PhD}$ thesis, Wageningen University, Wageningen, the Netherlands (2019) With references, with summaries in English, Dutch and Spanish.

ISBN: 978-94-6343-567-3

DOI: https://doi.org/10.18174/467275 
Para mi familia y todos aquellos que me hicieron sentir como en casa, fuera donde fuese.

To my family and to those that made me feel at home, wherever home may had been. 
...1 seem to have been only like a boy playing on the seashore, and diverting myself in now and then finding a smoother pebble or a prettier shell than ordinary,

whilst the great ocean of truth lay all undiscovered before me.

Isaac Newton. 


\section{Contents}

\section{Chapter 1}

General introduction.

\section{Chapter 2}

Virus-induced gene silencing (VIGS) of meiotic genes in plants.

\section{Chapter 3}

Efficient reverse breeding by VIGS-mediated

transient crossover reduction.

\section{Chapter 4}

VIGS-mediated increase of recombination in Arabidopsis thaliana.

\section{Chapter 5}

A protocol for the downregulation of meiotic genes using virus-induced gene silencing (VIGS) in Arabidopsis thaliana.

\section{Chapter 6}

General discussion.

$\begin{array}{ll}\text { Acknowledgements } & 153\end{array}$

$\begin{array}{ll}\text { Curriculum Vitae } & 158\end{array}$

$\begin{array}{ll}\text { Publications } & 159\end{array}$ 



\section{Chapter 1}

\section{General introduction}




\section{The relevance of understanding and manipulating meiosis for plant breeders.}

Meiosis is the process that generates four haploid spores from a diploid cell through two consecutive cell divisions (Zickler and Kleckner, 2015). Meiosis is also a primary source of genetic variation between individuals in a population. The generation of new allele combinations is possible thanks to the process of meiotic recombination. Meiotic recombination occurs through the formation of crossovers (COs) between homologous chromosomes. Crossover resolution leads to the reciprocal exchange of genetic information between homologs (i.e. non-sister chromatids), hence creating new allele combinations (Mercier et al., 2015).Therefore, controlling meiosis means ultimate control over the genetic diversity that is generated and transmitted to the progeny.

The number of COs that occur in meiosis is tightly regulated through different and independent pathways. This means that, for example, a loss of function in the negative regulators of crossover formation leads to an increase in crossover frequencies. Increasing the number of recombination events with respect to wild-type meiosis is one of the major goals of modern plant breeding. It may boost the generation of novel allele combinations by increasing the chances of placing a crossover between linked genes that rarely recombine (Wijnker et al., 2012; Choi, 2017). To ensure meiotic progression, different proteins are required to act on the onset of the two meiotic divisions. Mutants of genes acting in either the first or the second meiotic division, produce diploid, instead of haploid spores, and may lead to increased ploidy in the offspring.

Several techniques can be used to obtain phenotypes derived from non-wild-type meiosis. Mutants have been the most exploited resource for plant breeders to improve phenotypes and they are still the primary tool used to characterize gene function. Mutants show a stable phenotype and can often be maintained over generations. However, mutants in some meiotic genes lead to inviable spores or semi-sterility, so the use of recessive or dominant mutations in meiotic genes that cause infertility may not be used in commercial breeding lines (Zhang et al., 2017; Mieulet et al., 2018). Other techniques such as RNAi technology can be used to knock down a meiotic gene and lead to the generation of meiotic phenotypes (Wijnker et al., 2014; Casacuberta et al., 2015) although some species are recalcitrant to stable transformation and hence, this technique cannot be considered as a universal applicable tool/approach (Senthil-Kumar and Mysore, 2011). A transient silencing approach such as virus-induced gene silencing (VIGS) would allow the induction of a phenotype without permanently modifying the plant genome. VIGS is a method that allows delivery of dsRNAs to the plant which can then trigger the activation of the immune system response leading to the downregulation of an endogenous target sequence (Hamilton and Baulcombe, 1999; Ratcliff, MartinHernandez and Baulcombe, 2001; Burch-Smith, Miller and Dinesh-Kumar, 2006; Padmanabhan and Dinesh-Kumar, 2009). To do so, the target sequence is cloned into 
a viral vector that is then inoculated in the plant. As long as the virus is active, the gene is downregulated, but once the plant overcomes the viral infection, the induced phenotype is no longer present and the plant reverts to its wild-type phenotype (BurchSmith et al., 2004). The use of VIGS to knock down any putative gene in meiosis could be of great help to produce offspring that its transgene-free in only one generation (Dirks et al., 2009).

The development of VIGS-for-meiosis will be of great help to rapidly obtain meiotic phenotypes, but the potential and limitations of the method can be first evaluated in a model organism such as Arabidopsis thaliana to evaluate its feasibility later in crops. Arabidopsis thaliana represents the preferred model organism for the study of meiosis in plants. This is not surprising when considering the characteristics of this plant species. Arabidopsis is a small plant, with a short life cycle (about eight weeks on average), and a single plant sets thousands of seeds, facilitating the production of transformants or mutant offspring from single individuals (Somerville and Koornneef, 2002). A single flower produces several hundreds of pollen grains that can be easily collected either to phenotype and/or to generate offspring and/or to study phenotypic variation in the gametes. Arabidopsis also has one of the smallest genomes among flowering plants, which allows the rapid generation of genetic resources such as insertion mutants or the identification of polymorphisms between accessions (Somerville and Koornneef, 2002). Arabidopsis is closely related to other members of the Brassicaceae, which greatly facilitates the translation of technological advances to Brassicas, such as cruciferous vegetables, cabbages, or mustard plants. The use of Arabidopsis thaliana as a model organism in research has provided the plant research community with a large number of well characterized meiotic mutants, allowing the fast identification of orthologous genes in crops. Thus, Arabidopsis serves as a model not only to characterize meiotic mutant phenotypes but also to identify which methods are more efficient to alter meiosis (i.e, stable transformation, abiotic agents, transient silencing approaches). In this thesis I investigate the potential of VIGS to become a versatile method to control meiosis in plants, leading to the efficient application of breeding technologies. To illustrate this, I first provide an overview about meiosis, focusing on the initiation and progression of meiotic recombination and the regulation of the two meiotic divisions, as these two features condition genetic variation. Then, I explain some of the applications that had been developed using meiotic mutant phenotypes. In addition, I indicate which methods have been regularly used to induce mutant phenotypes in plants and compare them to VIGS, considering the advantages and disadvantages of all different methods in the context of plant breeding. I then describe the underlying molecular mechanism of VIGS by explaining the immune system response to the VIGS system based on the Tobacco rattle virus, (TRV), the most common viral vector used in Arabidopsis. Finally, I will briefly outline the various chapters in my thesis. 


\section{Meiosis; the unique cell process that determines genetic diversity.}

2.1 Two different pathways regulate meiotic recombination in plants.

Meiotic recombination is the main process driving genetic variation. Recombination is initiated by the formation of double-strand breaks (DSBs), executed by the SPO-11PRD1-MTOPVIB (SPORULATION-11; PUTATIVE RECOMBINATION INITIATION DEFECT 1; MEIOTIC TOPOISOMERASE VIB-LIKE) containing complex (De Muyt et al., 2007; Lambing, Franklin and Wang, 2017; Tang et al., 2017). In Arabidopsis, it is estimated that about 150-200 DSBs occur in meiosis, generating free DNA ends distributed along the chromosome arms (Vignard et al., 2007; Serrentino and Borde, 2012) (figure 1). DSBs are hazardous for the cell and their complete repair is crucial for preserving genome integrity. The DSB repair mechanism is first initiated when the DNA ends are nicked and resected producing 3' ssDNA overhangs (Puchta, 2005; Lambing, Franklin and Wang, 2017). After that, these ssDNAs invade homologous sequences, which can be either found in the sister chromatid or in the homologous chromatid (Puchta, 2005). This process is mediated by the recombinases DISRUPTED MEIOTIC CDNA 1 (DMC1) and RADIATION SENSITIVE 51 (RAD51) (Da Ines et al., 2013), which dynamics and numbers are regulated by a conserved complex formed by FIDGETIN LIKE-1 (FIGL) and FIDGETIN-LIKE-1 INTERACTING PROTEIN (FLIP) FIGL1-FLIP (Girard et al., 2015; Fernandes et al., 2018). Fernandes et al., 2018 suggested that the FIGL1-FLIP complex suppresses the repair of DSBs through interhomolog strand invasion, possibly promoting a DSBs repair mechanism using the sister chromatid as a template instead (see figure 1). DSBs repair through inter-homolog strand invasion leads to the formation of recombination intermediates that can be repaired as non-crossovers (NCO) or crossovers (CO) (Wijnker et al., 2013; Lambing, Franklin and Wang, 2017). In wild-type meiosis the number of COs is generally confined to 1-2 COs per homologous pair (Wijnker and de Jong, 2008). Crossovers are essential to ensure correct chromosome pairing and stabilize and promote balanced chromosome segregation at meiosis I (Jones and Franklin, 2006).

Two different types of COs coexist in plant meiosis (Higgins et al., 2004; Mercier et al., 2005). The Class I CO pathway accounts in Arabidopsis for about $80 \%-85 \%$ of the total number of COs (Higgins et al., 2004; Mercier et al., 2005). Type I COs are interference-sensitive, so the occurrence of one crossover impedes the presence of another crossover nearby (Higgins et al., 2004; Mercier et al., 2005; Lambing, Franklin and Wang, 2017). Hence, class I COs tend to be placed further apart in the chromosomes arms (Higgins et al., 2008; Lu et al., 2008; Falque et al., 2009; Zhang et al., 2014; Lambing, Franklin and Wang, 2017). The class I CO pathway is regulated by ZMM proteins (figure 1) (Lynn, Soucek and Börner, 2007). Among the ZMM proteins, ZINC-REGULATED TRANSPORTERS IRON-REGULATED TRANSPORTER-LIKE PROTEIN 1 (ZIP1), MUTS-HOMOLOG 4;5 (MSH4-MSH5), and MEIOTIC 
RECOMBINATION 3 (MER3) are actively involved in the stabilization of recombination intermediates while MUT-L HOMOLOGS 1;3 (MLH1- MLH3) are required for maturation of these into COs (see figure 1) (Mercier et al., 2005; Dion et al., 2007; Lynn, Soucek and Börner, 2007; Higgins et al., 2008; Phillips et al., 2013; Zhang et al., 2014; Choi, 2017). Moreover, the partners of the RTR complex (TOP3alfa-RMI1-RECQ4A) mediate that recombination intermediates are exclusively resolved as NCOs (Knoll, Schröpfer and Puchta, 2014). However, inter-homolog intermediates can also be repaired through the MMS AND UV SENSITIVE 81 (MUS81) pathway or through a process called singledependent strand annealing (SDSA). Class II COs are interference-insensitive and depend on the MUS81 pathway, accounting for roughly $20 \%-15 \%$ of the total number of COs in Arabidopsis (Berchowitz et al., 2007; Falque et al., 2009; Knoll et al., 2012). The Class II CO pathway is negatively regulated by FANCONI ANEMIA COMPLEMENTATION GROUP M-LIKE PROTEIN (FANCM), which stimulates that most of the SDSA intermediates are resolved as NCOs instead. In the absence of FANCM, these intermediates are resolved by MUS81, leading to an increase of the total class II COs (see figure 1) (Knoll et al., 2012; Girard et al., 2015).

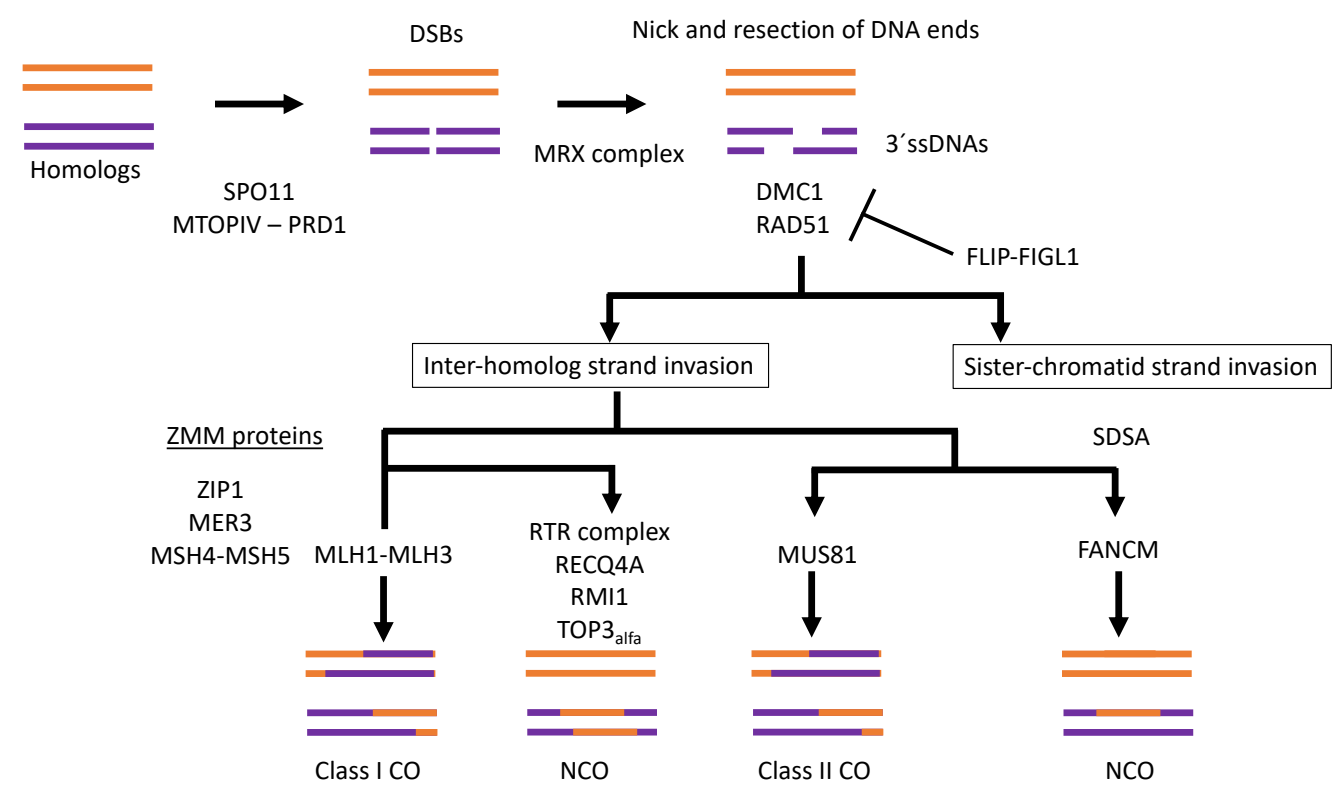

Figure 1. Crossover (CO) formation and its regulation in meiosis. DSBs are always initiated by the SPO11 containing complex. DSBs must be then repaired using a homologous template, which is done through the process of sister chromatid strand invasion or the inter-homolog strand invasion. This process is regulated by the essential recombinases DMC1 and RAD51 which dynamics are also controlled by FLIP-FIGL1, possibly limiting that DSBs are repaired through inter-homolog strand invasion. If DSBs are repaired via inter-homolog strand invasion, recombination intermediates are formed and DSBs are finally repaired as non-crossovers (NCO) or crossovers (COs). COs can be generated though two different pathways. The ZMM protein pathway regulates class I COs, a type of 
COs that are interference-dependent. At the same time, the RTR complex promotes the resolution of DSBs as NCO via the ZMM protein pathway. On the other hand, class II COs are initially derived from the formation of SDSA (synthesis-dependent strand annealing). Class II COS are interference independent and are processed by MUS81, which action is possibly counteracted by FANCM, that promotes the resolution of DSBs as NCO instead.

\subsection{Two consecutive meiotic divisions lead to the formation of haploid gametes.}

Meiotic progression requires a specialized regulation of the cell cycle.

Two consecutive cell divisions are a unique feature of meiotic cells, and hence require a dedicated and specialized cell division regulation. Meiotic progression occurs thanks to the complementing activity of CDKs (CYCLIN DEPENDENT KINASES) and cyclins and the interaction of those with essential meiotic proteins that act on the onset of meiosis I and meiosis II (See review Wijnker and Schnittger 2013). In addition, oscillating levels of CDKs and cyclins promoting cell cycle progression are simultaneously regulated by the APC/C (ANAPHASE PROMOTING COMPLEX/CYCLOSOME) (Wijnker and Schnittger, 2013). Fluctuations between high CDK activity versus low APC/C activity are necessary for the progression of meiosis I and meiosis II; For the first and the second meiotic division to happen, CDK activity has to reach a certain threshold that is only possible if concomitantly the activity of the APC/C level decreases. After the second meiotic division takes place, CDK activity decreases again and ultimately cytokinesis will lead to the formation of four independent haploid spores (Wijnker and Schnittger, 2013).

Discrete protein-protein interactions are necessary to ensure meiotic progression at different steps during the cell cycle. Furthermore, different CDKs-cyclines complexes control meiotic processes that are specific of male or female meiosis. For instance, the cyclin SDS (SOLO DANCERS) is required for homologue pairing and crossover formation while another cycline, TAM (TARDY ASYNCHRONOUS MEIOSIS) is required from meiotic progression only in male meiosis (Azumi et al., 2002; d'Erfurth et al., 2010). Also, different regulators of the APC/C activity exist in meiosis (Wijnker and Schnittger, 2013). For instance, OSD1/GIG1 (OMISSION OF SECOND MEIOTIC DIVISSION I/GIGAS CELL1), reduces APC/C activity to promote exclusively the onset of meiosis II in both, male and female meiosis (Cromer et al., 2012). The mentioned processed can only roughly shape the complexity of the regulatory mechanism of meiosis, but it shows how if very specific targets in meiosis are silenced, a large variety of phenotypes can be potentially induced. Furthermore, selected targets of female and male meiosis can be used to obtained biased effects to modify genetic inheritance in one direction.

\section{The cohesin complex is essential for chromosome integrity.}

Genetic variation is not only determined by crossover formation but also by the different combinations of parental chromosomes that will segregate to form haploid gametes. For this process to occur, the cohesin complex must ensure structural integrity during 
monopolar chromosome orientation and segregation (Watanabe and Nurse, 1999; Peters, Tedeschi and Schmitz, 2008; Zamariola et al., 2014). Specifically, the perfectly coordinated spatial and temporal removal of one of the main subunits of this complex, REC8, permits that firstly only homologs and secondly chromatids separate (Watanabe and Nurse, 1999). Before homologs segregate, REC8 is removed from the chromosome arms but protected at the centromeres to hold the sister chromatids together (Watanabe and Nurse, 1999). After that, cytoskeleton fibres attach to kinetochores on the centromeric regions, pulling each of the homologs to opposite poles (Chelysheva et al., 2005). At the onset of meiosis II, REC8 is finally removed from the centromeres and sister chromatids separate to form haploid spores. Because of the pulling forces and originated tensions between the cytoskeleton and the chromosomes in each of the meiotic divisions, REC8 is essential to secure structural chromosome integrity. REC8 is also essential to maintain cohesion during DSBs repair. A single rec8 mutant typically suffers from chromosome fragmentation and chromosome bridges, visible from diakinesis to anaphase I due to the existence of unrepaired DSBs (Chelysheva et al., 2005).

\section{Modification of meiosis for plant breeding.}

Potentially, all the previously described meiotic mechanisms can be altered to change genetic inheritance to the offspring, especially when genes directly involved in class I or class II CO pathway are targeted. In the following sections, I discuss several plant breeding methods that benefited from the use of meiotic mutant phenotypes. This first section will not only explain the applications derived from a modification on $\mathrm{CO}$ frequencies for efficient hybrid breeding, but also how plant breeders can profit from such alteration to obtain mapping populations. Furthermore, I explain polyploid production based on the reduction of the number of meiotic divisions. Finally, I describe how by combining three meiotic mutants one can induce a mitotic like division in meiosis (MiMe), which receives the name of synthetic apomeoisis.

3.1 Changes in recombination frequencies for efficient -hybrid- breeding and the generation of mapping populations.

\section{Decrease of recombination frequencies to preserve desired allelic combinations.}

Efficient production of superior or heterotic varieties is the ultimate goal of plant breeders. Heterosis is inherent to hybrid plants, as in the hybrid new allelic combinations provided by two distinct parental genomes may cause an enhanced phenotype for the trait of interest compared to the respective parents (Schnable and Springer, 2013). To produce commercial hybrids, inbred (homozygous) lines should be first generated in a process that usually takes up to several generations of self-fertilization or backcrossing (Robsa Shuro, 2017). This can be extremely lengthy, especially in species with long generation time. The process to generate inbred lines can be shortened if haploid and double haploid technology is available for the crop of interest (Forster et al., 2007; Britt 
and Kuppu, 2016; Ren et al., 2017). The possibility of regenerating haploid gametes as haploid lines allows to, after an event of genome doubling, these haploid plants set seed, giving rise to homozygous double-haploids (Ravi and Chan, 2010; Ren et al., 2017; Robsa Shuro, 2017). Yet, haploid and DH technology has not been developed for all species, such as in tomato, or an extremely low haploid and $\mathrm{DH}$ regeneration efficiency makes its implementation unfeasible in large breeding set-ups (Seguí-Simarro and Nuez, 2007; Ren et al., 2017). Not only the production of inbred lines require a large investment of resources; the performance of produced hybrids should also be tested and assessed in field trials to evaluate its potential as a commercial hybrid.

Reverse breeding is a technology developed to produce hybrids more efficiently compared to traditional plant breeding methods (Dirks et al., 2009). Instead of relying on the time-consuming generation of inbred lines, reverse breeding allows one to obtain homozygous breeding lines directly from an exceptionally performing hybrid. Then, the hybrid can be regenerated by crossing two complementing homozygous lines (Dirks et al., 2009; Wijnker et al., 2012). Wijnker et al. $(2012,2014)$ provided the proof of concept for this technology in Arabidopsis thaliana. The original design of reverse breeding was based on complete suppression of $\mathrm{COs}$ in a $\mathrm{F} 1$ hybrid using a dominant acting RNAi transgene against $D M C 1$, hence preserving the two parental genotypes (figure 1). In the total absence of COs, non-recombinant chromosomes randomly segregate at meiosis I (Couteau et al., 1999; Da Ines et al., 2013). By chance, very few of the meiocytes will have their non-recombinant chromosomes segregating in a balanced manner. This small fraction of viable gametes can be regenerated as haploids (Wijnker et al., 2012, 2014). Consequently, a DH population derived from an achiasmatic meiosis, will be formed by homozygous lines of distinct parental combinations among which, perfectly complementing pairs can be selected to reconstitute the initial heterozygote (Dirks et al., 2009; Wijnker et al., 2014).

Unfortunately, the original reverse breeding design presented two main drawbacks for its efficient application in crops. First, it requires that one of the parental lines is transformed with a dominant RNAi transgene to achieve downregulation of COs later in the hybrid. Random segregation of chromosomes then occurs due to the absence of COs, leading to mostly aneuploid gametes. Secondly, as recombination is essential for balanced chromosome segregation, the probability of finding balanced gametes in complete absence of recombination is very small. This means that the more chromosomes are segregating randomly, the lower the chance is of finding balanced gametes (Dirks et al., 2009). This probability can be calculated as (1/2)x: in Arabidopsis $(x=5)$ the proportion of viable gametes equals $(1 / 2)^{5} .100=3 \%$ while in maize $(x=10)$ this fraction as low as $(1 / 2)^{10} .100=0,10 \%$. Dirks et al., 2009 proposed that to overcome these drawbacks, one could use a method to directly downregulate gene expression in a wild-type hybrid, avoiding stable transformation methods. Furthermore, reverse breeding could be feasible in species with high chromosome numbers if low 
recombination levels are allowed and so less homologs segregate randomly in meiosis (Dirks et al., 2009; Wijnker et al., 2012). For instance, if the class I CO pathway is downregulated in Arabidopsis, a remaining 15\%-20\% COs rate will still occur, which results in approximately 1.5 - 2 COs per meiosis (Mercier et al., 2005; Higgins et al., 2008). Assuming one mandatory $C O$ per homologous pair in Arabidopsis, recombinant chromosomes will segregate in a balanced way, increasing the chances to find viable gametes from $3 \%$ - complete crossover suppression- to $(1 / 2)^{5-2}=12,5 \%$ - incomplete crossover suppression. Similarly, a decrease of recombination frequencies versus a complete crossover suppression will be expected to increase the probability of finding viable gametes in species with a high number of chromosomes.

Reverse breeding generates chromosome substitution lines (CSLs).

Reverse breeding is not only useful to efficiently produce hybrids, but it can also be used to obtain mapping populations (Wijnker et al., 2012; Wijnen et al., 2018). Reverse breeding generates a population of homozygous lines in which different combinations of non-recombinant parental chromosomes will be found in the offspring. The method of introgressing chromosomes of one parental genotype into the background of the other parental genotype result in the production of a series of chromosome substitution lines/strains (CSLs) (Nadeau et al., 2000). The substitution can affect either one chromosome (single chromosome substitution line) or multiple chromosomes. When all the possible combinations are generated, a full chromosome substitution library is obtained. CSLs form a type of mapping population that can be used to assess chromosome-chromosome interactions, for QTL mapping (at chromosome level) or to dissect genome wide epistasis (Wijnen et al., 2018). The efficient generation of CSLs in crops could greatly help the identification of specific chromosome combinations that explain the phenotypic variation of heterotic traits. Through reverse breeding, a complete chromosome substitution library (32 lines) can be only obtained in two generations from the F1 hybrid (Wijnker et al., 2012).

\section{Increase of recombination frequencies can speed up plant breeding.}

An increase of recombination frequencies is one of the most valuable tools for plant breeders, as it leads to an increment of crossover events in the population. An increase of recombination frequencies is directly translated in the generation of multiple allelic combinations that otherwise will take much longer to be found in a wild-type meiosis (Fernandes et al., 2017; Mieulet et al., 2018). The increase of recombination frequencies can be used to enhance QTL mapping resolution using populations such as recombinant inbred lines (RILs) (Balasubramanian et al., 2009; Takuno, Terauchi and Innan, 2012). RILs are traditionally obtained by first crossing two parental lines to generate F1 offspring and later progressing through several generations of inbreeding. This allows to eventually fix specific allelic combinations in an genetically "immortal" homozygous background that can then be used permanently as a mapping population (Keurentjes et 
al., 2007; Takuno, Terauchi and Innan, 2012). However, the production of RILs is lengthy - usually needs seven-eight generations (Seymour et al., 2012) and also demands considerable investment of resources, especially in crops such as maize (long generation times and large greenhouse / field space). In addition, these populations require a minimum size of about 50-250 individuals to guarantee a good mapping resolution (Collard et al., 2005). Yet, resolution is limited by the amount of recombination that occurs during the creation of RIL populations (Korte and Farlow, 2013). To increase mapping resolution one can either enlarge the population size or alternatively, in a given population size, increase recombination frequencies (Wijnker and De Jong, 2008, Fernandes et al. 2017).

Thanks to the research done in Arabidopsis thaliana, it is known that an increase of crossover frequencies can be achieved through increasing the number of both class I or class II COs (Wang et al., 2012; Fernandes et al., 2017; Serra et al., 2018). Since class II COs are interference independent, two crossovers can occur close to each other in theory in any region in the chromosome (Copenhaver, Housworth and Stahl, 2002; Mercier et al., 2005; Berchowitz et al., 2007; Zamariola et al., 2014). Hence, an increase in type II COs is a powerful approach to generate more possible allele combinations. In plants, class II COs are negatively regulated by at least three parallel pathways, controlled by FIGL1-FLIP, RECQ4 and FANCM (Crismani et al., 2012; Girard et al., 2014, 2015; Zamariola et al., 2014; Séguéla-Arnaud et al., 2015; Choi, 2017; Fernandes et al., 2017; Zhang et al., 2017; Mieulet et al., 2018). Single mutants of either one of these genes in Arabidopsis display a significant increase in recombination frequencies compared to a wild-type meiosis.

The combination of some of the mutations in FIGL1, RECQ4 and FANCM leads to an additive increase of crossover frequencies in both homozygous and hybrid backgrounds (Crismani et al., 2012; Girard et al., 2015; Séguéla-Arnaud et al., 2015; Fernandes et al., 2017). However, although these negative regulatory pathways are conserved in plants, mutants of the mentioned genes display distinct phenotypes among different plant species. The figl1 and fancm mutant phenotypes observed in crops do not entirely mimic the mutant phenotypes in Arabidopsis: mutants of FIGL1 show complete infertility in rice and pea but not in Arabidopsis, while fancm does not cause an increase of recombination frequencies in tomato and Arabidopsis hybrids but it does in rice, pea, and Brassicas (Blary et al., 2018; Mieulet et al., 2018).

The increase of recombination frequencies in meiosis holds great potential in the field of plant breeding but a better characterization of these anti-crossover pathways in both, meiotic and somatic cells is required to ensure recovery of high-recombinant offspring in different plant species. For this, different methods (targeted mutagenesis to generate complete knockouts, hypomorphic alleles, knockdowns) should be assessed 
to validate if lines that show an increase of recombination can be used in a breeding setup.

3.2 Modification of the number of meiotic divisions for polyploid production.

The importance of meiotic mutants to produce polyploids.

Polyploidy has had a major impact on the evolution of wild- and cultivar species (Sattler, Carvalho and Clarindo, 2016). In many cases, polyploids exhibit proven superior performance that can relate to enlarged organs/yield and heterosis in polyploid hybrids (Comai, 2005). Furthermore, polyploid hybrids benefit from the so called "genomic buffer", which reduces the effect of deleterious mutations and often present a higher tolerance to both abiotic and biotic stress (Sattler, Carvalho and Clarindo, 2016). Polyploids can arise either through events of whole genome duplication that affect either the meristem or the zygote (Chen, 2010; De Storme and Geelen, 2011) or through the production of unreduced gametes (D'Erfurth et al., 2008; Brownfield and Köhler, 2011; Herben, Trávníček and Chrtek, 2016; Sattler, Carvalho and Clarindo, 2016).

Unreduced gametes are obtained when either the first or the second meiotic division does not occur (meiotic restitution). If a first division restitution (FDR) takes place, anaphase $\mathrm{I}$ is skipped and sister chromatids suffer premature separation, leading to highly heterozygous chromosomes, while in a second division restitution (SDR) anaphase II is skipped and gametes containing chromosomes that are homozygous in the centromere region are formed. Because of crossover formation and chromosome segregation (in SDR) the genetic makeup of the gametes is different (Sattler, Carvalho and Clarindo, 2016). In true FDR gametes homologs fail to pair, crossover formation is impaired, and eventually non-recombinant chromatids segregate in meiosis II. SDR gametes on the other hand execute meiosis I, but because the second meiotic division does not take place, the two sister chromatids of each homolog do not segregate. Yet, since the first meiotic division occurred, these gametes will carry recombinant chromosomes.

Several meiotic mutants have been characterized for the production of diploid gametes. For instance, jason mutants show $2 \mathrm{n}$ gamete production caused by a first meiotic restitution and the formation of a parallel spindle which leads to nuclear restitution after meiosis II. Mutants of PS1 produce 2n gamete formation also due to a defect of the spindle formation in meiosis II. In both cases, diploid gametes formed in jason or ps1 backgrounds carry recombinant chromosomes (De Storme and Geelen, 2011). in tam and osd1, 2n gametes are formed through a second meiotic restitution in which the second meiotic division does not take place, leading to the production of SDR (D'Erfurth et al., 2009; d'Erfurth et al., 2010). The mutant dyad in Arabidopsis has been shown to produce true FDR gametes, and hence one of the major components of apomixis: apomeiosis - or bypass of meiosis (Ravi, Marimuthu and Siddiqi, 2008; Hand and Koltunow, 2014). In any case, fully functional $2 n$ gametes are able to carry out 
fertilization and can give rise to polyploid offspring (D'Erfurth et al., 2008, 2009; Ravi, Marimuthu and Siddiqi, 2008; d'Erfurth et al., 2010; De Storme and Geelen, 2011).

Turning meiosis into mitosis: A method for synthetic apomeiosis.

Recent studies (D'Erfurth et al., 2009; Mieulet et al., 2016) showed that synthetic apomeiosis in plants is also possible in Arabidopsis and in a crop such as rice (Oryza sativa) by combining the mutation of three genes in meiosis: absence of DSBs formation and asynaptic meiosis (Atspo11; Ospair1), early segregation of sister chromatids at meiosis I due to loss of cohesion (Atrec8; Osrec8), and formation of diploid gametes by second meiotic restitution (Atosd1 ; Ososd1) (Watanabe and Nurse, 1999; Stacey et al., 2006; d'Erfurth et al., 2010; Mieulet et al., 2016). In a spo11 rec8 mutant background, sister chromatids separate prematurely in meiosis I but due to the absence of DSBS (spo11), there is no chromosome fragmentation in rec8 (Chelysheva et al., 2005; D'Erfurth et al., 2009). Then, osd1 rescues the spo11/pair1 rec8 phenotype as no second meiotic division takes place, and so balanced segregation of sister chromatids in meiosis I leads to the formation of viable diploid gametes. The resulting genotype was named MiMe (Mitosis instead of Meiosis) and leads to a high percentage of viable FDR gametes.

\section{Techniques to modify meiosis.}

Several techniques have been described to date to induce mutant phenotypes in meiosis (Lambing, Franklin and Wang, 2017). They can influence somatic and/or meiotic processes with or without affecting gene structure and gene expression. Here, I present the main treatments used in plants and compare their advantages and disadvantages. Furthermore, I introduce virus-induced gene silencing as a potential tool to regulate gene expression in meiosis.

Different abiotic agents have been shown to modify recombination frequencies in meiosis and also induce formation of unreduced gametes. Chemicals such as DAMPAQ or puromycin have been reported to abolish crossover formation while UV-radiation increase recombination frequencies (Ries et al., 2000; Sánchez-Morán et al., 2004). Yet, these methods are undirected and affect the whole genome unrestrictedly, potentially leading to undesired mutations. The application of these chemicals or UV-radiation can cause undesired effects not only during meiosis but also by affecting somatic tissues (Ries et al., 2000; Kodym and Afza, 2003) and they do not work as a universal method to target all the possible meiotic genes. Other abiotic treatments like cold or heat shock (De Storme, Copenhaver and Geelen, 2012; Wang et al., 2017) and chemicals like colchicine or nitrous oxide (NO) (Forster et al., 2007; Younis, Hwang and Lim, 2014) have been described as efficient approaches to promote diploid gamete production, but they have not been generally established as breeding tools for stably polyploid generation (De Storme, Copenhaver and Geelen, 2012; Wang et al., 2017; Liu, De Storme and Geelen, 2018). In addition, severe developmental problems can derive from 
these treatments. Cold or heat-shock can negatively affect pollen viability, anther structure, and flower and fruit development (Prasad, J. Boote and Allen, 2006; BolañosVillegas, Jane and Jauh, 2010; Giorno et al., 2013; Harsant et al., 2013). The use of colchicine can be accompanied by potential and severe side-effects (M. Al-Khayri, Mohan Jain and V. Johnshon, 2016). Plants treated with colchicine are often weakened and produced polyploid offspring display great variability (Brownfield and Köhler, 2011; M. Al-Khayri, Mohan Jain and V. Johnshon, 2016). Colchicine affects genome stability, causes mitotic abnormalities and changes in chromosome structure (Rodriguez et al., 2001; Kundu and Ray, 2017). Hence, colchicine-derived polyploids could exhibit additional meiotic defects.

Mutants are still the primary source of genetic variation/diversity in plant breeding and they represent the preferred resource used to identify and characterize gene function. For instance, at least 3,200 mutant varieties of more than 224 species have been released worldwide (M. Al-Khayri, Mohan Jain and V. Johnshon, 2016). Mutants are usually obtained through stable transformation techniques such as T-DNA insertions that cause a change in gene structure. Production of T-DNA collections are for instance available for rice and Arabidopsis (TAIR;RiceGE). Chemical mutagenesis (TILLING and EMS populations), gene editing methods such as the CRISPR/cas9 system or the use of TALENS can also cause constitutive mutations. The benefit of using mutations in breeding lines is that it allows fixing a desirable trait in different backgrounds (M. AlKhayri, Mohan Jain and V. Johnshon, 2016). Yet, when it comes to meiotic mutants, their use in breeding lines is not that straightforward. For example, some of the mutations in meiotic genes can cause high rates of male and/or female abortion and a severe decrease in fertility (Dion et al., 2007; Lu et al., 2008; Da Ines et al., 2013; Lambing, Franklin and Wang, 2017; Zhang et al., 2017).

Gene silencing techniques, such as RNAi technology, have also been repeatedly used as a targeted method to silence genes in meiosis (Moritoh et al., 2005; Dirks et al., 2009; Brownfield and Köhler, 2011; Wijnker et al., 2012; Casacuberta et al., 2015). Another approach recently described to downregulate genes, consists of spraying leaves with dsRNAs that can penetrate leaf tissues, spread systemically and silence endogenous target sequences in the plant (Tang, 2013; Dalakouras et al., 2016). Yet, this technique has not been shown to efficiently downregulate endogenous genes for longer than few days, nor has it been shown to work for meiotic genes. Virus-induced gene silencing (VIGS) is a technique that is regularly used to downregulate plant genes. It is based on a viral system delivered into the plant that carries a fragment homologous to the host target sequence (Ratcliff, Martin-Hernandez and Baulcombe, 2001). The plant immune system initiates a response that ultimately promotes the degradation of both the virus and mRNA of the plant target gene.

VIGS may more efficiently induce mutant phenotypes in meiosis as compared to previously mentioned strategies (Bennypaul et al., 2012; Bhullar et al., 2014). The 
successful downregulation of a meiotic gene can be in many cases verified by simple pollen phenotyping (i.e. pollen abortion/diploid gamete production) (Peterson, Slovin and Chen, 2010; De Storme and Geelen, 2011). VIGS is able to cause a phenotype in only 3-4 weeks in Arabidopsis after agroinoculation (Burch-Smith, 2006), meaning a great time-saving alternative over the production of gene knockouts and knockdowns through RNAi technology or generation of mutants via T-DNA insertions or CRISPR/Cas9, which usually takes (at least) two generations. Another benefit of using VIGS over stable transformation is that one can chose a viral system in which the silencing effect is, or is not, transmitted to the progeny of the species of choice (Senthil-Kumar and Mysore, 2011). In addition, the virus inducing a specific phenotype can also be eliminated using a heat or freeze shock (Wang and Valkonen, 2008; Wang et al., 2008; Senthil-Kumar and Mysore, 2011). This makes VIGS to date the only transient silencing approach that allows assessing gene functions that would be embryo or seedling-lethal in a mutant background (T. M. Burch-Smith 2006; Becker, 2013). Finally, VIGS is an attractive alternative to rapidly induce a phenotype in plants that are recalcitrant to stable transformation (Becker and Lange, 2009; Senthil-Kumar and Mysore, 2011).

VIGS exploits the PTGS (post-transcriptional gene silencing) response of the plant to downregulate endogenous sequences through a vial system (Ratcliff, MartinHernandez and Baulcombe, 2001; Liu et al., 2002; Susi et al., 2004). Although the genomes of many viruses have been engineered to be used a VIGS vectors, here I focus on the Tobacco rattle virus or TRV for being the most widely used to silence genes in Arabidopsis (Ratcliff, Martin-Hernandez and Baulcombe, 2001; Brigneti et al., 2004; Velasquez, Chakravarthy and Martin, 2009; Sahu et al., 2012). The silencing mechanism is mediated by a modified viral vector that carries a homologous sequence to an endogenous plant target (VIGS construct). This VIGS construct is usually first cloned into Agrobacterium tumefaciens and then delivered into the plant for instance, through agroinoculation by leaf-infiltration (Burch-Smith et al., 2004, 2006; Vaghchhipawala et al., 2011) (see figure 2). Once Agrobacterium infects and transforms one or several cells of the local tissue successfully and the integrated T-DNA is transcribed and translated, the resulting virus initiates a round of autonomous replication and propagation assisted by a second viral vector, TRV1, that encodes for the replication protein complex essential for systemic spread within the host (Ratcliff, Martin-Hernandez and Baulcombe, 2001; Becker and Lange, 2009). The vector will then be transcribed into an active RNA virus. The virus, during its transcription- and replication processes will produce dsRNA molecules as intermediates (Watson et al., 2005) which are recognized as viral molecules by the immune system of the plant. This activates a response to degrade the dsRNA into 21-24 nt siRNA via DICER-like proteins like (Hamilton and Baulcombe, 1999; Susi et al., 2004; Watson et al., 2005; Becker, 2013). Once processed into 21-24nt molecules, double-stranded siRNAs are melted into singlestranded siRNAs that are loaded onto ARGONAUTE, a subunit of RISC, leading the 
complex to mRNAs that are homologous to the siRNA (Susi et al., 2004; FernandezPozo et al., 2015). The recognition triggers degradation of the mRNAs leading to a posttranscriptional silencing of the target gene and of the virus (Susi et al., 2004). In this way, both, viral RNA and host mRNA homologous to the produced 21-24 nt siRNAs are silenced (Burch-Smith et al., 2004) (see figure 2).

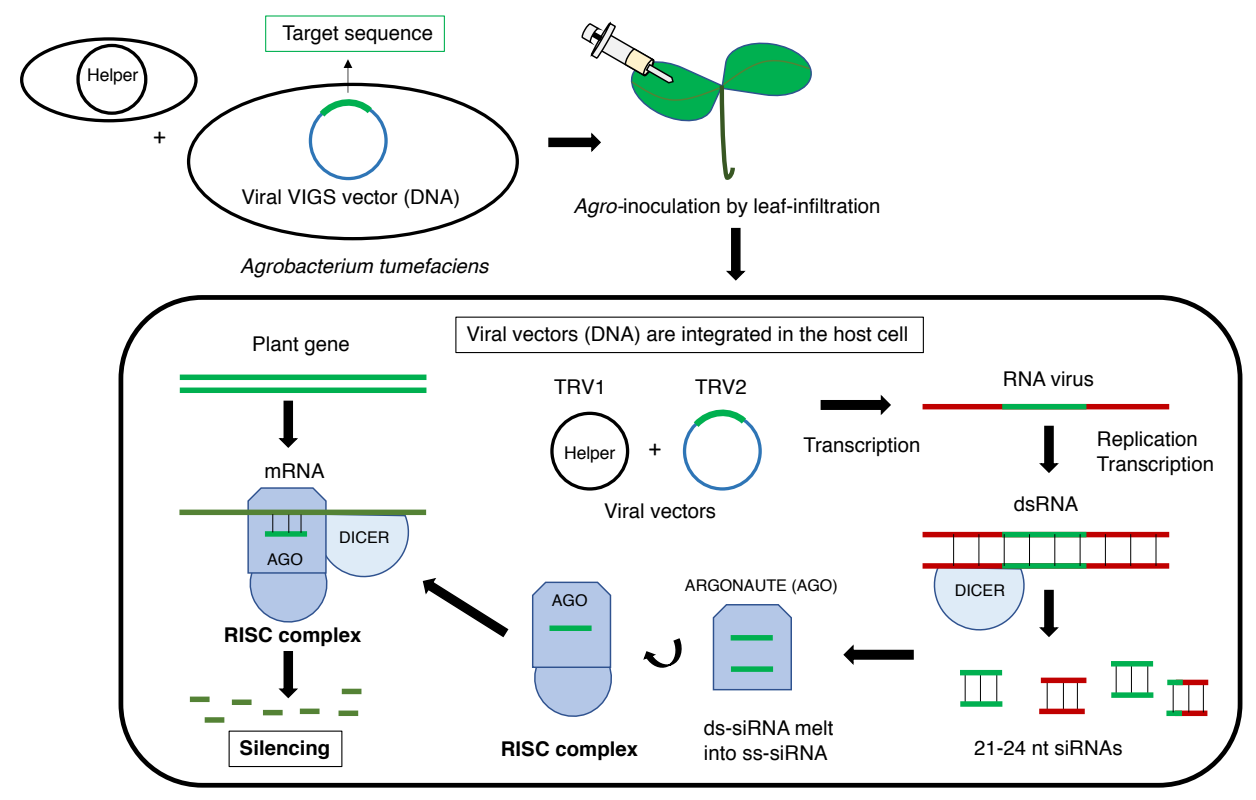

Figure 2. VIGS triggers a post-transcriptional silencing response leading to silencing of the target gene. Upon Agrobacterium inoculation and T-DNA (vector) integration, transcription and translation, dsRNAS are derived from an actively replicating RNA virus that activates the immune RISC complex. The dsRNAs are processed by DICER-like proteins into 21-24 nucleotide- double-stranded siRNAs, which are then recognized and loaded as single stranded siRNAS by ARGONAUTE, a subunit of RISC, leading the complex to mRNAS homologous to the siRNAS (Becker and Lange, 2009). Upon homologous recognition, the mRNAs are processed into small pieces, causing the silencing of the target gene.

\section{Outline of the thesis.}

In this thesis I describe how I set out to establish VIGS as a method to efficiently downregulate genes to manipulate the process of meiosis in plants. I couple VIGS to the development of new breeding technologies using Arabidopsis thaliana as a model organism (i.e., reverse breeding, production of polyploids). By doing this, I shall be able to assess the advantages and limitations of using transient silencing approaches in crops.

In Chapter 2 I first explore the potential of VIGS to test the susceptibility of different genotypes to the technique by silencing endogenous genes in early and late flowering Arabidopsis accessions. Furthermore, I used VIGS to induce five meiotic and one post- 
meiotic phenotypes in Arabidopsis thaliana. I evaluate the efficiency of the technique by pollen phenotyping, plant fertility and ploidy level in the offspring. Finally, I test the possibility of downregulating three meiotic genes simultaneously and assess the phenotypes obtained.

In Chapter 3, I show that reverse breeding can be done in Arabidopsis thaliana by the use of VIGS. By simultaneously modifying the reverse breeding approach, I show the possibility of overcoming different limitations of the original reverse breeding design. I silenced $\mathrm{MSH} 5$ to reduce recombination frequencies in a wild-type F1 Col-Ler hybrid by approximately $80 \%$. I then show that reverse breeding through partial crossover suppression leads to the efficient recovery of non-recombinant DH (CSLs) and lowrecombinant $\mathrm{DH}$ offspring that can be used to first recreate the full hybrid and then create a population of distinct near-full hybrids. Thanks to the efficiency observed in Arabidopsis thaliana, I concur that the new design of the technique allows for the development of reverse breeding for crop species with higher chromosome number than Arabidopsis.

In Chapter 4, I test whether VIGS can be used to increase recombination frequencies by first silencing FIGL1 and RECQ4 in a $z m m$ background and then simultaneously knocking down RECQ4 and FIGL1. Although the meiotic cell spreads done in the "double knockdown" plants phenocopy the figl1 recq4 mutant in Arabidopsis, and thus show the feasibility of increasing recombination frequencies using VIGS, I was unable to obtain high recombinant offspring. In Chapter 4, I further explore the possible implications of working with either mutants and knockdowns and reconsider the assumed role of these anti-crossover factors as universal tools in plant breeding to generate high-recombinant offspring.

In Chapter 5 I provide a detailed protocol of a TRV2-VIGS system for meiosis in Arabidopsis thaliana. Although several VIGS protocols have been published, here I explain the modifications to these protocols to precisely adjust the inoculation time to target meiosis. Furthermore, I indicate how to verify a meiotic phenotype through chromosome spreads or through pollen phenotyping and provide with the general guidelines to obtain offspring from VIGS-derived gametes.

In Chapter 6, I discuss the future adaptation of VIGS-for-meiosis in several crops, focusing on the main elements that require attention to guarantee silencing in meiosis. I also evaluate the possibility of using VIGS to produce polyploids in a breeding set-up. I then explain how thanks to the experiments performed in Arabidopsis thaliana, reverse breeding has finally become feasible for crop breeding and the efficient generation of mapping populations.

Finally, ongoing experiments using VIGS to target meiotic genes in tomato revealed that strictly controlled growing conditions are required to faithfully assess a meiotic phenotype. The tomato plants used, displayed large variability in pollen phenotypes and 
therefore, validation of silencing of the target through pollen phenotyping is not possible using the current experimental set-up (Unpublished results; Linus Hohenwarter, master thesis, 2018).

\section{References and notes.}

1. Azumi, Y. et al. (2002) 'Homolog interaction during meiotic prophase I in Arabidopsis requires the SOLO DANCERS gene encoding a novel cyclin-like protein', The EMBO

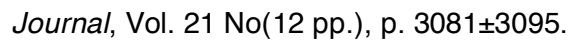

2. Balasubramanian, S. et al. (2009) 'QTL mapping in new Arabidopsis thaliana advanced intercross-recombinant inbred lines', PLOS ONE, 4(2), pp. 1-8. doi: 10.1371/journal.pone.0004318.

3. Becker, A. (2013) Virus-Induced Gene Silencing. Methods in Molecular Biology. Edited by A. Becker. Humana Press. doi: 10.1007/978-1-62703-278-0.

4. Becker, A. and Lange, M. (2009) 'VIGS - genomics goes functional', Trends in Plant Science, 15(1), pp. 1-4. doi: 10.1016/j.tplants.2009.09.002.

5. Bennypaul, H. S. et al. (2012) 'Virus-induced gene silencing (VIGS) of genes expressed in root, leaf, and meiotic tissues of wheat.', Functional and Integrative Genomics, 12(1), pp. 143-156. doi: 10.1007/s10142-011-0245-0.

6. Berchowitz, L. E. et al. (2007) 'The role of AtMUS81 in interference-insensitive crossovers in A. thaliana', PLoS Genetics, 3(8), pp. 1355-1364. doi: 10.1371/journal.pgen.0030132.

7. Bhullar, R. et al. (2014) 'Silencing of a metaphase l-specific gene results in a phenotype similar to that of the Pairing homeologous 1 ( Ph1) gene mutations', Proceedings of the National Academy of Sciences, 111(39), pp. 14187-14192. doi: 10.1073/pnas.1416241111.

8. Blary, A. et al. (2018) 'FANCM limits meiotic crossovers in Brassica crops', Frontiers in Plant Science, 9(368), pp. 1-13. doi: 10.3389/fpls.2018.00368.

9. Bolaños-Villegas, P., Jane, W.-N. and Jauh, G.-Y. (2010) 'Pollen development: A play with many actors', Plant Biology, 11. doi: 10.13140/2.1.5143.7124.

10. Brigneti, G. et al. (2004) 'Virus-induced gene silencing in Solanum species.', The Plant Journal, 39(2), pp. 264-272. doi: 10.1111/j.1365-313X.2004.02122.x.

11. Britt, A. B. and Kuppu, S. (2016) 'Cenh3: An emerging player in haploid induction technology', Frontiers in Plant Science, 7(357). doi: 10.3389/fpls.2016.00357.

12. Brownfield, L. and Köhler, C. (2011) 'Unreduced gamete formation in plants: Mechanisms and prospects', Journal of Experimental Botany, 62(5), pp. 1659-1668. doi: 10.1093/jxb/erq371.

13. Burch-Smith, T. M. et al. (2004) 'Applications and advantages of virus-induced gene silencing for gene function studies in plants.', Plant Journal, 39(5), pp. 734-746. doi: 10.1111/j.1365-313X.2004.02158.x.

14. Burch-Smith, T. M. et al. (2006) 'Efficient virus-induced gene silencing in Arabidopsis'', Plant Physiology, 142(1), pp. 21-27. doi: 10.1104/pp.106.084624.

15. Burch-Smith, T. M., Miller, J. L. and Dinesh-Kumar, S. P. (2006) 'Delivery of dsRNA into Plants by VIGS methodology', Cold Spring Harbor Protocols, 2006(1), p. pdb.prot4327pdb.prot4327. doi: 10.1101/pdb.prot4327.

16. Casacuberta, J. M. et al. (2015) 'Biotechnological uses of RNAi in plants: Risk assessment considerations', Trends in Biotechnology, 33(3), pp. 145-147. doi: 10.1016/j.tibtech.2014.12.003.

17. Chelysheva, L. et al. (2005) 'AtREC8 and AtSCC3 are essential to the monopolar orientation of the kinetochores during meiosis', Journal of Cell Science, 118(20), pp. 4621-4632. doi: 
$10.1242 / \mathrm{jcs} .02583$.

18. Chen, Z. J. (2010) 'Molecular mechanisms of polyploidy and hybrid vigor.', Trends in plant sciences, 15(2), pp. 57-71. doi: 10.1016/j.tplants.2009.12.003.Molecular.

19. Choi, K. (2017) 'Advances towards controlling meiotic recombination for plant breeding.', Molecules and cells, 40(11), pp. 814-822. doi: 10.14348/molcells.2017.0171.

20. Collard, B. C. Y. et al. (2005) 'An introduction to markers, quantitative trait loci (QTL) mapping and marker-assisted selection for crop improvement: The basic concepts', Euphytica, 142(1-2), pp. 169-196. doi: 10.1007/s10681-005-1681-5.

21. Comai, L. (2005) 'The advantages and disadvantages of being polyploid', Nature Reviews Genetics, 6(11), pp. 836-846. doi: 10.1038/nrg1711.

22. Copenhaver, G. P., Housworth, E. A. and Stahl, F. W. (2002) 'Crossover interference in Arabidopsis', Genetics, 160(4), pp. 1631-1639. doi: 10.1086/376610.

23. Couteau, F. et al. (1999) 'Random chromosome segregation without meiotic arrest in both male and female meiocytes of a dmc1 mutant of Arabidopsis.', The Plant Cell, 11(9), pp. 1623-1634. doi: 10.2307/3871042.

24. Crismani, W. et al. (2012) 'FANCM limits meiotic crossovers', Science, 336(6088), pp. 15881590. doi: $10.1126 /$ science. 1220381.

25. Cromer, L. et al. (2012) 'OSD1 promotes meiotic progression via APC/C inhibition and forms a regulatory network with TDM and CYCA1;2/TAM', PLoS Genetics, 8(7), pp. 1-14. doi: 10.1371/journal.pgen.1002865.

26. d'Erfurth, I. et al. (2010) 'The CYCLIN-A CYCA1;2/TAM is required for the meiosis I to meiosis II transition and cooperates with OSD1 for the prophase to first meiotic division transition', PLoS Genetics, 6(6), pp. 1-12. doi: 10.1371/journal.pgen.1000989.

27. D'Erfurth, I. et al. (2008) 'Mutations in AtPS1 (Arabidopsis thaliana Parallel Spindle 1) lead to the production of diploid pollen grains.', PLoS Genetics, 4(11), pp. 1-9. doi: 10.1371/journal.pgen.1000274.

28. D'Erfurth, I. et al. (2009) 'Turning meiosis into mitosis.', PLoS Biology, 7(6), p. e1000124. doi: 10.1371 /journal.pbio.1000124.

29. Dalakouras, A. et al. (2016) 'Induction of silencing in plants by high-pressure spraying of In vitro-synthesized small RNAs.', Frontiers in Plant Science, 7(1327), doi: 10.3389/fpls.2016.01327.

30. Dion, É. et al. (2007) 'An Arabidopsis MLH1 mutant exhibits reproductive defects and reveals a dual role for this gene in mitotic recombination', Plant Journal, 51(3), pp. 431-440. doi: 10.1111/j.1365-313X.2007.03145.x.

31. Dirks, R. et al. (2009) 'Reverse breeding: A novel breeding approach based on engineered meiosis', Plant Biotechnology Journal, pp. 837-845. doi: 10.1111/j.1467-7652.2009.00450.x.

32. Falque, M. et al. (2009) 'Two types of meiotic crossovers coexist in maize', The Plant Cell, 21(12), pp. 3915-3925. doi: 10.1105/tpc.109.071514.

33. Fernandes, J. B. et al. (2017) 'Unleashing meiotic crossovers in hybrid plants', Proceedings of the National Academy of Sciences, 115(10), pp. 2431-2436. doi: 10.1073/pnas.1713078114.

34. Fernandes, J. B. et al. (2018) 'FIGL1 and its novel partner FLIP form a conserved complex that regulates homologous recombination.', PLoS genetics, 14(4), p. e1007317. doi: 10.1371/journal.pgen.1007317.

35. Fernandez-Pozo, N. et al. (2015) 'The SGN VIGS tool: User-friendly software to design virus-induced gene silencing (VIGS) Constructs for functional genomics', Molecular Plant, 8(3), pp. 486-488. doi: 10.1016/j.molp.2014.11.024.

36. Forster, B. P. et al. (2007) 'The resurgence of haploids in higher plants', Trends in Plant 
Science, 12(8), pp. 368-375. doi: 10.1016/j.tplants.2007.06.007.

37. Giorno, F. et al. (2013) 'Ensuring reproduction at high temperatures: The heat stress response during anther and pollen development', Plants, 2(3), pp. 489-506. doi: 10.3390/plants2030489.

38. Girard, C. et al. (2014) 'FANCM-associated proteins MHF1 and MHF2, but not the other Fanconi anemia factors, limit meiotic crossovers.', Nucleic Acids Research, 42(14), pp. 9087-9095. doi: 10.1093/nar/gku614.

39. Girard, C. et al. (2015) 'AAA-ATPase FIDGETIN-LIKE 1 and helicase FANCM antagonize meiotic crossovers by distinct mechanisms.', PLoS Genetics, 11(7), pp. 1-22. doi: 10.1371/journal.pgen.1005369.

40. Hamilton, A. J. and Baulcombe, D. C. (1999) 'A Species of small antisense RNA in posttranscriptional gene silencing in plants', Science, 286(5441), pp. 950-952. doi: $10.1086 / 327380$.

41. Hand, M. L. and Koltunow, A. M. G. (2014) 'The genetic control of apomixis: Asexual seed formation', Genetics, 197(2), pp. 441-450. doi: 10.1534/genetics.114.163105.

42. Harsant, J. et al. (2013) 'High temperature stress and its effect on pollen development and morphological components of harvest index in the $\mathrm{C} 3$ model grass Brachypodium distachyon', Journal of Experimental Botany, 64(10), pp. 2971-2983. doi: 10.1093/jxb/ert142.

43. Herben, T., Trávníček, P. and Chrtek, J. (2016) 'Reduced and unreduced gametes combine almost freely in a multiploidy system', Perspectives in Plant Ecology, Evolution and Systematics, 18, pp. 15-22. doi: 10.1016/j.ppees.2015.12.001.

44. Higgins, J. D. et al. (2004) 'The Arabidopsis MutS homolog AtMSH4 functions at an early step in recombination: Evidence for two classes of recombination in Arabidopsis', Genes and Development, 18(20), pp. 2557-2570. doi: 10.1101/gad.317504.

45. Higgins, J. D. et al. (2008) 'AtMSH5 partners AtMSH4 in the class I meiotic crossover pathway in Arabidopsis thaliana, but is not required for synapsis', The Plant Journal, 55(1), pp. 28-39. doi: 10.1111/j.1365-313X.2008.03470.x.

46. Da Ines, O. et al. (2013) 'Meiotic recombination in Arabidopsis Is catalysed by DMC1, with RAD51 playing a supporting role', PLoS Genetics, 9(9), p. e1003787. doi: 10.1371/journal.pgen.1003787.

47. Jones, G. H. and Franklin, F. C. H. (2006) 'Meiotic Crossing-over: Obligation and Interference', Cell, 126(2), pp. 246-248. doi: 10.1016/j.cell.2006.07.010.

48. Keurentjes, J. J. B. et al. (2007) 'Development of a near-isogenic line population of Arabidopsis thaliana and comparison of mapping power with a recombinant inbred line population', Genetics, 175(2), pp. 891-905. doi: 10.1534/genetics.106.066423.

49. Knoll, A. et al. (2012) 'The Fanconi Anemia Ortholog FANCM ensures ordered homologous recombination in both somatic and meiotic cells in Arabidopsis', The Plant Cell, 24(4), pp. 1448-1464. doi: 10.1105/tpc. 112.096644.

50. Knoll, A., Schröpfer, S. and Puchta, H. (2014) 'The RTR complex as caretaker of genome stability and its unique meiotic function in plants', Frontiers in Plant Science, 5(33). doi: 10.3389/fpls.2014.00033.

51. Kodym, A. and Afza, R. (2003) 'Physical and chemical mutagenesis', in Grotewold, E. (ed.) Plant Functional Genomics. Methods in Molecular Biology. Totowa, NJ: Humana Press, pp. 189-203. doi: 10.1385/1-59259-413-1:189.

52. Korte, A. and Farlow, A. (2013) 'The advantages and limitations of trait analysis with GWAS: A review', Plant Methods, 9(29), pp. 1-9. doi: 10.1186/1746-4811-9-29.

53. Kundu, L. M. and Ray, S. (2017) 'Mitotic abnormalities and micronuclei inducing potentials of 
colchicine and leaf aqueous extracts of Clerodendrum viscosum Vent. in Allium cepa root apical meristem cells', Caryologia, 70(1), pp. 7-14.

54. Lambing, C., Franklin, F. C. H. and Wang, C.-J. R. (2017) 'Understanding and manipulating meiotic recombination in plants.', Plant Physiology, 173(3), pp. 1530-1542. doi: 10.1104/pp.16.01530.

55. Liu, B., De Storme, N. and Geelen, D. (2018) 'Cold-induced male meiotic restitution in Arabidopsis thaliana is not mediated by GA-DELLA Signaling', Frontiers in Plant Science, 9(February), pp. 1-11. doi: 10.3389/fpls.2018.00091.

56. Liu, H. et al. (2002) 'Functional replacement of the Tobacco rattle virus cysteine-rich protein by pathogenicity proteins from unrelated plant viruses.', Virology, 298(2), pp. 232-239. doi: 10.1006/viro.2002.1421.

57. Lu, X. et al. (2008) 'The Arabidopsis MutS homolog AtMSH5 is required for normal meiosis', Cell Research, 18(5), pp. 589-599. doi: 10.1038/cr.2008.44.

58. Lynn, A., Soucek, R. and Börner, G. V. (2007) 'ZMM proteins during meiosis: Crossover artists at work', Chromosome Research, 15(5), pp. 591-605. doi: 10.1007/s10577-007-11501.

59. M. Al-Khayri, J., Mohan Jain, S. and V. Johnshon, D. (2016) Advances in plant breeding strategies: breeding, biotechnology and molecular tools., Springer. Springer. doi: 10.1007/978-3-319-22521-0_7.

60. Mercier, R. et al. (2005) 'Two meiotic crossover classes cohabit in Arabidopsis: One is dependent on MER3, whereas the other one is not', Current Biology, 15(8), pp. 692-701. doi: 10.1016/j.cub.2005.02.056.

61. Mercier, R. et al. (2015) 'The molecular biology of meiosis in plants', Annual Review of Plant Biology, 66, pp. 297-327. doi: 10.1146/annurev-arplant-050213-035923.

62. Mieulet, D. et al. (2016) 'Turning rice meiosis into mitosis', Cell Research., 26(11), pp. 12421254. doi: $10.1038 / \mathrm{cr} .2016 .117$.

63. Mieulet, D. et al. (2018) 'Unleashing meiotic crossovers in crops', Nature plants ., 4(12), pp 1010-1016. doi 10.1038/s41477-018-0311-x

64. Moritoh, S. et al. (2005) 'RNAi-mediated silencing of OSGEN-L (OsGEN-like), a new member of the RAD2/XPG nuclease family, causes male sterility by defect of microspore development in rice', Plant and Cell Physiology, 46(5), pp. 699-715. doi: 10.1093/pcp/pci090.

65. De Muyt, A. et al. (2007) 'AtPRD1 is required for meiotic double strand break formation in Arabidopsis thaliana.', EMBO Journal, 26(18), pp. 4126-4137. doi: 10.1038/sj.emboj.7601815.

66. Nadeau, J. H. et al. (2000) 'Analysing complex genetic traits with chromosome substitution strains', Nature Genetics, 24(3), pp. 221-225. doi: 10.1038/73427.

67. Padmanabhan, M. and Dinesh-Kumar, S. P. (2009) 'Virus-induced gene silencing as a tool for delivery of dsRNA into plants', Cold Spring Harbor Protocols, 4(2). doi: 10.1101/pdb.prot5139.

68. Peters, J. M., Tedeschi, A. and Schmitz, J. (2008) 'The cohesin complex and its roles in chromosome biology', Genes and Development, 22(22), pp. 3089-3114. doi: 10.1101/gad.1724308.

69. Peterson, R., Slovin, J. P. and Chen, C. (2010) 'A simplified method for differential staining of aborted and non-aborted pollen grains', International Journal of Plant Biology, 1(2), pp. 66-69. doi: 10.4081/pb.2010.e13.

70. Phillips, D. et al. (2013) 'Quantitative high resolution mapping of HvMLH3 foci in barley pachytene nuclei reveals a strong distal bias and weak interference', Journal of Experimental 
Botany, 64(8), pp. 2139-2154. doi: 10.1093/jxb/ert079.

71. Prasad, P. V. V., J. Boote, K. and Allen, L. (2006) 'Adverse high temperature effects on pollen viability, seed-set, seed yield and harvest index of grain-sorghum [Sorghum bicolor

(L.) Moench] are more severe at elevated carbon dioxide due to higher tissue temperatures', Agricultural and Forest Meteorology, 139(3-4), pp. 237-251. doi:

10.1016/j.agrformet.2006.07.003.

72. Puchta, H. (2005) 'The repair of double-strand breaks in plants: Mechanisms and consequences for genome evolution', Journal of Experimental Botany, 56(409), pp. 1-14. doi: $10.1093 / \mathrm{jxb} / \mathrm{eri025}$.

73. Ratcliff, F., Martin-Hernandez, A. M. and Baulcombe, D. C. (2001) 'Tobacco rattle virus as a vector for analysis of gene function by silencing', Plant Journal, 25(2), pp. 237-245. doi: 10.1046/j.0960-7412.2000.00942.x.

74. Ravi, M. and Chan, S. W. L. (2010) 'Haploid plants produced by centromere-mediated genome elimination', Nature., 464(7288), pp. 615-618. doi: 10.1038/nature08842.

75. Ravi, M., Marimuthu, M. P. A. and Siddiqi, I. (2008) 'Gamete formation without meiosis in Arabidopsis', Nature, 451(7182), pp. 1121-1124. doi: 10.1038/nature06557.

76. Ren, J. et al. (2017) 'Novel technologies in doubled haploid line development', Plant Biotechnology Journal, 15(11), pp. 1361-1370. doi: 10.1111/pbi.12805.

77. Ries, G. et al. (2000) 'UV-damage-mediated induction of homologous recombination in Arabidopsis is dependent on photosynthetically active radiation', Proceedings of the National Academy of Sciences, 97(24), pp. 13425-13429. doi: 10.1073/pnas.230251897.

78. Robsa Shuro, A. (2017) 'Approaches in developing inbred lines in cross-pollinated crops', Biochemistry and Molecular Biology, 2(4), p. 40. doi: 10.11648/j.bmb.20170204.12.

79. Rodriguez, E. M. et al. (2001) 'Colchicine promotes a change in chromosome structure without loss of sister chromatid cohesion in prometaphase l-arrested bivalents.', Chromosoma., 110(7), pp. 478-486. doi: 10.1007/s004120100162.

80. Sahu, P. P. et al. (2012) 'Recent advances in tomato functional genomics: Utilization of VIGS', Protoplasma, 249(4), pp. 1017-1027. doi: 10.1007/s00709-012-0421-7.

81. Sánchez-Morán, E. et al. (2004) 'A puromycin-sensitive aminopeptidase is essential for meiosis in Arabidopsis thaliana.', The Plant cell, 16(11), pp. 2895-2909. doi: 10.1105/tpc.104.024992.

82. Sattler, M. C., Carvalho, C. R. and Clarindo, W. R. (2016) 'The polyploidy and its key role in plant breeding', Planta., 243(2), pp. 281-296. doi: 10.1007/s00425-015-2450-x.

83. Schnable, P. S. and Springer, N. M. (2013) 'Progress toward understanding heterosis in crop plants', Annual Review of Plant Biology, 64(1), pp. 71-88. doi: 10.1146/annurev-arplant042110-103827.

84. Séguéla-Arnaud, M. et al. (2015) 'Multiple mechanisms limit meiotic crossovers: TOP3a and two BLM homologs antagonize crossovers in parallel to FANCM', Proceedings of the National Academy of Sciences, 112(15), pp. 4713-4718. doi: 10.1073/pnas.1423107112.

85. Seguí-Simarro, J. M. and Nuez, F. (2007) 'Embryogenesis induction, callogenesis, and plant regeneration by in vitro culture of tomato isolated microspores and whole anthers', Journal of Experimental Botany, 58(5), pp. 1119-1132. doi: 10.1093/jxb/erl271.

86. Senthil-Kumar, M. and Mysore, K. S. (2011) 'New dimensions for VIGS in plant functional genomics', Trends in Plant Science. 16(12), pp. 656-665. doi: 10.1016/j.tplants.2011.08.006.

87. Serra, H. et al. (2018) 'Massive crossover elevation via combination of $H E / 10$ and recq4a recq4b during Arabidopsis meiosis.', Proceedings of the National Academy of Sciences, 115(10), pp. 2437-2442. doi: 10.1073/pnas.1713071115. 
88. Serrentino, M. E. and Borde, V. (2012) 'The spatial regulation of meiotic recombination hotspots: Are all DSB hotspots crossover hotspots?', Experimental Cell Research., 318(12), pp. 1347-1352. doi: 10.1016/j.yexcr.2012.03.025.

89. Seymour, D. K. et al. (2012) 'Rapid creation of Arabidopsis doubled haploid lines for quantitative trait locus mapping', Proceedings of the National Academy of Sciences, 109(11), pp. 4227-4232. doi: 10.1073/pnas.1117277109.

90. Somerville, C. and Koornneef, M. (2002) 'A fortunate choice: The history of Arabidopsis as a model plant', Nature Reviews Genetics, 3(11), pp. 883-889. doi: 10.1038/nrg927.

91. Stacey, N. J. et al. (2006) 'Arabidopsis SPO11-2 functions with SPO11-1 in meiotic recombination', The Plant Journal, 48(2), pp. 206-216. doi: 10.1111/j.1365313X.2006.02867.x.

92. De Storme, N., Copenhaver, G. P. and Geelen, D. (2012) 'Production of diploid male gametes in Arabidopsis by cold-induced destabilization of postmeiotic radial microtubule arrays', Plant Physiology, 160(4), pp. 1808-1826. doi: 10.1104/pp.112.208611.

93. De Storme, N. and Geelen, D. (2011) 'The Arabidopsis mutant jason produces unreduced first division restitution male gametes through a parallel/fused spindle mechanism in meiosis II', Plant Physiology, 155(3), pp. 1403-1415. doi: 10.1104/pp.110.170415.

94. Susi, P. et al. (2004) 'Characteristics of RNA silencing in plants: similarities and diferences across kingdoms', Plant Molecular Biology, 54(2), pp. 157-174.

95. Takuno, S., Terauchi, R. and Innan, H. (2012) 'The power of QTL mapping with RILs', PLoS ONE, 7(10), p. e46545. doi: 10.1371/journal.pone.0046545.

96. Tang, G. Q. (2013) 'Methods and compositions for introduction of exogenous dsrna into plant cells'. Google Patents. Available at: https://www.google.com/patents/CA2842709A1?cl=en.

97. Tang, Y. et al. (2017) 'MTOPVIB interacts with AtPRD1 and plays important roles in formation of meiotic DNA double-strand breaks in Arabidopsis', Scientific Reports, 7(1), pp. 1-13. doi: 10.1038/s41598-017-10270-9.

98. Vaghchhipawala, Z. et al. (2011) 'Agroinoculation and Agroinfiltration: Simple tools for complex gene function analyses.', in A., P. (ed.) Plant reverse genetics. Methods in Molecular Biology. Humana Press, Totowa, NJ, pp. 65-76. doi: 10.1007/978-1-60761-682-5.

99. Velasquez, A., Chakravarthy, S. and Martin, G. B. (2009) 'Virus-induced Gene Silencing (VIGS) in Nicotiana benthamiana and Tomato', Journal of Visualized Experiments, (28). doi: 10.3791/1292.

100. Vignard, J. et al. (2007) 'The interplay of RecA-related proteins and the MND1-HOP2 complex during meiosis in Arabidopsis thaliana', PLoS Genetics, 3(10), pp. 1894-1906. doi: 10.1371/journal.pgen.0030176.

101. Wang, J. et al. (2017) 'High temperature-induced production of unreduced pollen and its cytological effects in Populus', Scientific Reports. Springer US, 7(5281). doi: 10.1038/s41598-017-05661-x.

102. Wang, K. et al. (2012) 'The role of rice HEI10 in the formation of meiotic crossovers', PLoS Genetics, 8(7), pp. 1-13. doi: 10.1371/journal.pgen.1002809.

103. Wang, Q. et al. (2008) 'Combined thermotherapy and cryotherapy for efficient virus eradication: Relation of virus distribution, subcellular changes, cell survival and viral RNA degradation in shoot tips', Molecular Plant Pathology, 9(2), pp. 237-250. doi: 10.1111/j.1364-3703.2007.00456.x.

104. Wang, Q. C. and Valkonen, J. P. T. (2008) 'Elimination of two viruses which interact synergistically from sweetpotato by shoot tip culture and cryotherapy', Journal of Virological Methods, 154(1-2), pp. 135-145. doi: 10.1016/j.jviromet.2008.08.006. 
105. Watanabe, Y. and Nurse, P. (1999) 'Cohesin Rec8 is required for reductional chromosome segregation at meiosis', Nature, 400(6743), pp. 461-464. doi: 10.1038/22774.

106. Watson, J. M. et al. (2005) 'RNA silencing platforms in plants', FEBS, 579(26), pp. 59825987. doi: 10.1016/j.febslet.2005.08.014.

107. Wijnen, C. L. et al. (2018) 'A complete chromosome substitution mapping panel reveals genome-wide epistasis in Arabidopsis', bioRxiv. Available at: http://biorxiv.org/content/early/2018/10/05/436154.abstract.

108. Wijnker, E. et al. (2012) 'Reverse breeding in Arabidopsis thaliana generates homozygous parental lines from a heterozygous plant', Nature Genetics, 44(4), pp. 467-470. doi: 10.1038/ng.2203.

109. Wijnker, E. et al. (2013) 'The genomic landscape of meiotic crossovers and gene conversions in Arabidopsis thaliana', eLife, 17(2), pp. 1-22. doi: 10.7554/eLife.01426.

110. Wijnker, E. et al. (2014) 'Hybrid recreation by reverse breeding in Arabidopsis thaliana', Nature Protocols, 9(4), pp. 761-772. doi: 10.1038/nprot.2014.049.

111. Wijnker, E. and de Jong, H. (2008) 'Managing meiotic recombination in plant breeding', Trends in Plant Science, 13(12), pp. 640-646. doi: 10.1016/j.tplants.2008.09.004.

112. Wijnker, E. and Schnittger, A. (2013) 'Control of the meiotic cell division program in plants', Plant Reproduction, 26(3), pp. 143-158. doi: 10.1007/s00497-013-0223-x.

113. Younis, A., Hwang, Y.-J. and Lim, K.-B. (2014) 'Exploitation of induced 2n-gametes for plant breeding', Plant Cell Reports, 33(2), pp. 215-223. doi: 10.1007/s00299-013-1534-y.

114. Zamariola, L. et al. (2014) 'Chromosome segregation in plant meiosis', Frontiers in Plant Science, 5(279), pp. 1-19. doi: 10.3389/fpls.2014.00279.

115. Zhang, L. et al. (2014) 'Crossover formation during rice meiosis relies on interaction of OsMSH4 and OsMSH5', Genetics, 198(4), pp. 1447-1456. doi: 10.1534/genetics.114.168732.

116. Zhang, P. et al. (2017) 'The rice AAA-ATPase OsFIGNL1 Is essential for male meiosis', Frontiers in Plant Science, 8(1639), pp. 1-17. doi: 10.3389/fpls.2017.01639.

117. Zickler, D. and Kleckner, N. (2015) 'Recombination, pairing, and synapsis of homologs during meiosis', Cold Spring Harbor Perspectives in Biology, 7, pp. 1-20. doi: 10.1101/cshperspect.a016626. 
To new experiences.

\author{
"A garden is a grand teacher. \\ It teaches patience and careful watchfulness; \\ it teaches industry and thrift; above all it teaches entire trust."
}

Gertrude Jekyll 


\section{Chapter 2}

\section{Virus-induced gene silencing (VIGS) of meiotic genes in plants.}

\section{Authors.}

Vanesa Calvo-Baltanás', Nils Schönbeck², Bastiaan de Snoo ${ }^{3}$, Hans de Jong1', Arp Schnittger², Erik Wijnker'1.

\section{Author affiliations.}

1) Laboratory of Genetics, Wageningen University \& Research, P.O. Box 166700 AA Wageningen, The Netherlands.

2) Department of Developmental Biology, University of Hamburg, Institute for plant sciences and microbiology, 22609 Hamburg, Germany.

3) Rijk Zwaan R\&D Fijnaart, Eerste Kruisweg 9, 4793 RS Fijnaart, The Netherlands 


\begin{abstract}
.
Virus-induced gene silencing (VIGS) is a transient approach to modulate gene expression in plants. VIGS is a rapid alternative to induce mutant phenotypes compared to other stable transformation methods. In this study, we explored the potential of VIGS to modify meiosis in Arabidopsis thaliana. To test the versatility of the method, we targeted genes with different functions in meiosis. For instance, we could induce unreduced gamete production by silencing of $O S D 1$. Furthermore, we phenocopied the mutant pollen phenotypes described for rec8 (loss of chromatid cohesion) prd1, dmc1, sds mutants (absence of double-strand breaks and crossover formation). We also induced pollen tetrad formation by targeting QRT1 and QRT2. In addition, we constructed a VIGS vector targeting simultaneously REC8-PRD1-OSD1 to induce a $\mathrm{MiMe}$ (Mitosis instead of Meiosis) phenotype. While single knockdowns could be easily established, we encountered difficulties to simultaneously silencing three targets. This work shows the versatility of VIGS to induce various single meiotic phenotypes in Arabidopsis thaliana, and future efforts should focus on improving the approach for multiple gene silencing.
\end{abstract}

\title{
Introduction.
}

In recent years, there has been rising interest in modifying plant meiosis for breeding strategies (d'Erfurth et al., 2009; Dirks et al., 2009; Wijnker et al., 2012; Lambing, Franklin and Wang, 2017). This has in part been fuelled by the discovery of mutants in meiotic processes in model species. For instance, restitution of the first or the second meiotic divisions may lead to unreduced gamete formation and subsequently, to polyploid progeny (D'Erfurth et al., 2008; Brownfield and Köhler, 2011). Genetic engineering for increase of crossover frequencies may enable to generate mapping populations with higher mapping resolution (Crismani et al., 2012; Wang et al., 2012; Fernandes et al., 2017; Blary et al., 2018; Mieulet et al., 2018). In contrast, when recombination is completely suppressed, gametes carrying non-recombinant chromosomes may be produced at low frequencies. This type of gametes can be utilized for reverse breeding (Dirks et al., 2009; Wijnker et al., 2012). Other meiotic phenotypes that have promising potential for breeders, include the mutations that cause loss of chromatid cohesion (rec8), loss of double-strand break formation (DSBs) (spo11/prd1) and a second meiotic restitution (osd1). The combination of these mutations leads to the formation of unreduced gametes that are genetically identical to the mother cell. This phenotype receives the name of MiMe (Mitosis instead of Meiosis) and it was initially shown in Arabidopsis and later applied in rice. The introgression of these three distinct meiotic mutations in the same background enables synthetic apomeiosis, one of the main components of apomixis. This is a major advancement towards a promising technology in which clonal reproduction for hybrid maintenance of crops can become 
feasible in a breeding set-up (d'Erfurth et al., 2009; Hand and Koltunow, 2014; Mieulet et al., 2016).

Several meiotic mutants that are involved in the same pathway can display similar phenotypes. Therefore, manipulation of different meiotic genes can lead to analogous phenotypes that are useful for plant breeding. For instance, since homologous recombination is required for balanced chromosome segregation, mutants involved in crossovers formation exhibit univalents segregating at meiosis I and pollen abortion due to the formation of aneuploid gametes (Couteau et al., 1999; Azumi et al., 2002; De Muyt et al., 2007; Dion et al., 2007; Vrielynck et al., 2016). Mutants defective in various processes of meiosis, including chromosome pairing, DSBs initiation, DSB repair and crossover resolution, ultimately show partial or total lack of chiasma formation. The mutant dmc1 (DISRUPTED MEIOTIC CDNA 1) is required to repair DSBs through the formation of crossovers, so the absence of DMC1 protein leads to a failure in crossover formation (Da Ines et al. 2013). PRD1 (PUTATIVE RECOMBINATION INITIATION DEFECT 1) together with MTOPVIB (MEIOTIC TOPOISOMERASE VIB-LIKE) form an heterodimer that promote SPO11 (SPORULATION 11) dependent DSBs formation (De Muyt et al., 2007; Vrielynck et al., 2016). Another example is MLH1, (MUTLHOMOLOGUE 1) that plays a dual role in DNA repair mechanisms, not only in meiosis but also in mitosis where it is required for homologous recombination. The absence of $\mathrm{MLH} 1$ leads to a $72 \%$ reduction in homologous recombination in Arabidopsis (Dion et al., 2007). Mutants of SDS (SOLO DANCERS) fail to promote homolog interaction leading to incomplete synapsis and chiasma formation (Azumi et al., 2002). A second group of meiotic modifications involves the production of unreduced (mostly diploid - $2 n$ ) gametes (Brownfield and Köhler, 2011). Diploid gametes can be observed when a second meiotic restitution takes place, which occurs in an osd1 mutant background (d'Erfurth et al., 2009, 2010; Cromer et al., 2012). OSD1 promotes meiotic progression from meiosis I to meiosis II by the inhibition of the APC/C (Anaphase Promoting Complex/Cyclosome) (Cromer et al., 2012). The production of unreduced gametes can be easily identified by a pollen size that is larger in these mutants in comparison to the wildtype (De Storme et al., 2013).

The model plant Arabidopsis thaliana has played a crucial role in identifying and characterizing meiotic genes and phenotypes, setting up the stage for their characterization in other plants, including crops. The generation of these mutant phenotypes has been possible thanks to techniques that allow manipulation of gene structure or gene expression. Such techniques in plants are under continuous development, focusing on improving existing methods and exploring novel concepts and technologies. Stable transformation, which is the most common technology to insert alien sequences, is often time-consuming due to long plant generation time or even impossible, as some species or genotypes are recalcitrant to stable transformation (Becker and Lange, 2009; Senthil-Kumar and Mysore, 2011). In such cases, virus- 
induced gene silencing (VIGS) offers a promising alternative for efficiently conducting functional genetic analysis or to induce a desired phenotype.

VIGS exploits the immune system of the plant to transiently downregulate endogenous genes (Liu et al., 2002; Burch-Smith, 2006; Becker and Lange, 2009; Bennypaul et al., 2012). A large number of viral genomes had been modified to become VIGS vectors and used to efficiently downregulate genes in several plants species (Burch-Smith, 2006; Senthil-Kumar and Mysore, 2011; Becker, 2013). For instance, the ssRNA virus, TRV (Tobacco rattle virus) has been commonly used to silence genes in Arabidopsis (Brigneti et al., 2004; Burch-Smith, 2006; Unver and Budak, 2009). The TRV-based VIGS system consist of a modified bipartite viral genome, meaning that two vectors, TRV1 and TRV2 must be co-expressed in the plant simultaneously to guarantee the formation of an active virus (Ratcliff, Martin-Hernandez and Baulcombe, 2001; Burch-Smith et al., 2004). Once in the plant, the viral transcription and replication process generates dsRNA molecules that triggers an immune response through the activation of the RISC complex (Ruiz, Voinnet and Baulcombe, 1998; Baulcombe, 2004). dsRNAs will be first processed into siRNAs molecules and then loaded into the RISC complex. Homologous sequences to the siRNAs will be recognized as components of the viral genome, which ultimately causes the cleavage of the virus and the endogenous mRNA target at the same time (Becker and Lange, 2009; Unver and Budak, 2009). In VIGS, the target gene is downregulated within few days but the silencing is not fully penetrant (not all the cells in the host will be affected by the silencing) and the penetrance of the silencing phenotype varies (the strength of the silencing phenotype is different between treated plants). In addition, the virus is only stable for a short period of time (few weeks) after which, the induced phenotype cannot be observed anymore (Burch-Smith et al., 2004).

In this research we investigated the possibility of using VIGS to downregulate meiotic genes in different accessions of Arabidopsis thaliana. As a control, we knocked down PHYTOENE DESATURASE (PDS), which induces clear photobleaching symptoms in inoculated plants (Burch-Smith et al., 2006). In addition, we tested if a post-meiotic pollen phenotype could be induced. To do so, we targeted two QUARTET(QRT) genes: $Q R T 1$ and $Q R T 2$. The mutants qrt1 and qrt2 keep the four spores together after meiosis, allowing pollen-tetrad analysis of male meiosis in Arabidopsis (Preuss, Rhee and Davis, 1994; Rhee and Somerville, 1998; Copenhaver, Keith and Preuss, 2000; Francis, Lam and Copenhaver, 2006). We also explored the validation of meiotic phenotypes caused by VIGS through pollen phenotyping and through the recovery of polyploid offspring. In addition, we tested if simultaneous silencing of meiotic genes can be achieved using a single TRV vector. The results obtained allow to understand the potential of VIGS to downregulate several meiotic processes but also to gain insight about the possible limitations of VIGS-for-meiosis. 


\section{Results.}

1. VIGS efficiently downregulates gene expression in reproductive tissues of Arabidopsis.

As a proof of principle and to develop a positive control of VIGS-for-meiosis, we tested if TRV could induce silencing of $P D S$ in reproductive tissues in Arabidopsis plants. To do so, we inoculated respectively five seedlings of Columbia-0 (Col-0) and 26 of Landsberg erecta (Ler) with TRV2-PDS, 21 days post germination (hereafter d.p.g). We observed VIGS-induced photobleaching in the rosette leaves, main stem, siliques, flowers and flower buds at 40 days post inoculation (hereafter d.p.i). Figure 1 shows a mosaic of white sectors in green ones, indicating that the penetrance of the white phenotype in inoculated plants was not complete.
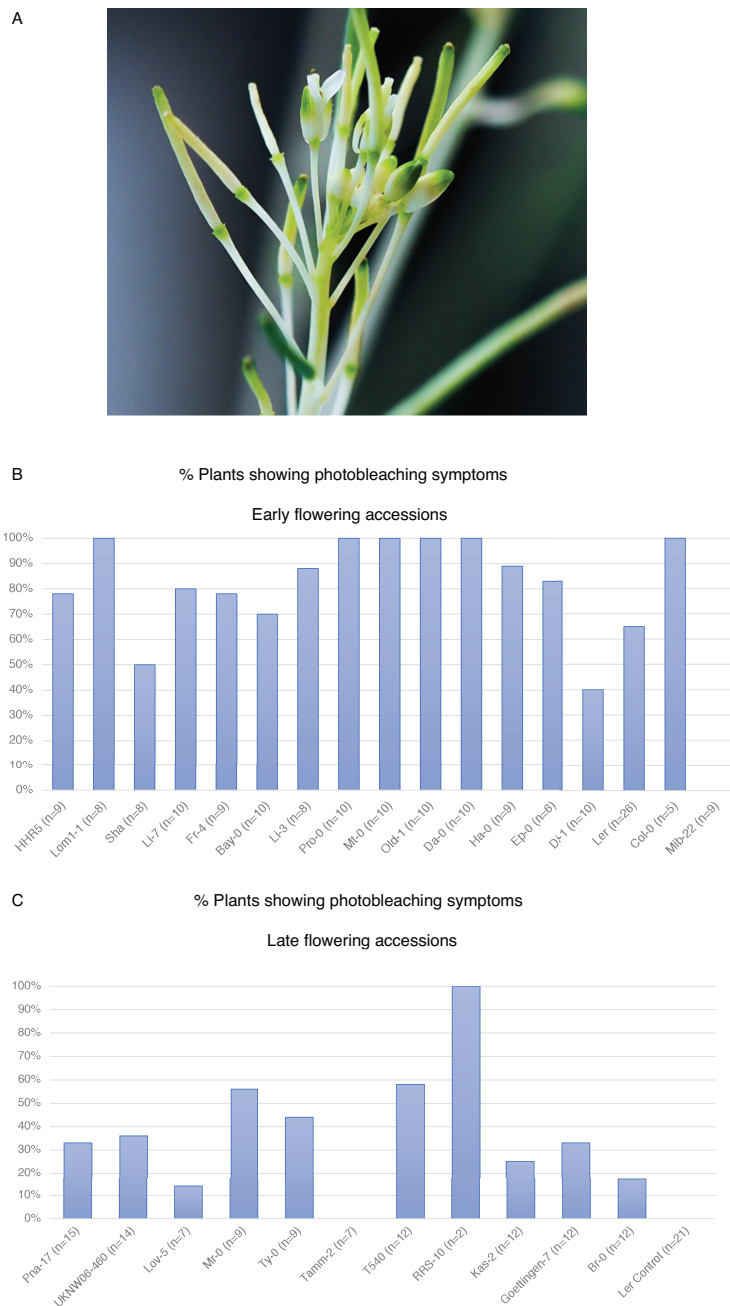
Figure 1. Silencing of $P D S$ in Arabidopsis thaliana. A) Depicts a Col-0 plant at 40 d.p.i where the silencing of $P D S$ has affected vegetative and reproductive tissues in the plant, illustrated by white siliques and flower buds. The graphs in B) and C) represent the frequency of plants (\%) of early and late flowering accession of Arabidopsis thaliana displaying photobleaching symptoms after inoculation with TRV2-PDS. The number of plants showing photobleaching symptoms were scored at 28 d.p.i in early flowering accessions and at four and five w.p.i in late flowering accessions. The number of total plants inoculated in each subset of plants $(n)$ is indicated in each case.

To investigate whether different Arabidopsis accessions respond similarly to our VIGS treatment, we inoculated Arabidopsis seedlings of 15 early flowering accessions (i.e., lines that require no vernalization for flowering) at 21 d.p.g. under standard growing conditions. Col-0 and Ler were included again as positive controls (Figure 1). The first signs of photobleaching appeared 12 d.p.i. The fraction of plants showing photobleaching was scored at 28 d.p.i. Signs of $P D S$ silencing were observed in 14 accessions, but the number of affected plants varied strongly between accessions. For example, all Col-0 plants showed photobleaching to none in the genotype Mib-22.

We also asked how other conditions different to the standard conditions used in our experimental set-ups would affect the penetrance of the silencing phenotype (see materials and methods). To test this, in a parallel experiment, we grew 11 Arabidopsis accessions which require a vernalization-cold treatment to shorten time to enter in flowering transition (Minorsky, 2003) (hereafter referred as late-flowering accessions). We then wondered whether the silencing of $P D S$ could be induced at low temperatures when plants were grown at $4^{\circ} \mathrm{C}$ after inoculation. We tested this by inoculating seedlings at 13 d.p.g with TRV2-PDS, including Col-0 plants and Ler plants used as controls. The late flowering accessions were first grown under standard conditions and transferred to a $4^{\circ} \mathrm{C}$ chamber one day after the inoculation, together with 21 Ler controls, while Col-0 remained under the standard growing conditions, so they could serve as positive controls of the infection. The first signs of $P D S$ silencing in Col-0 appeared at 10 d.p.i., while in the late flowering accessions plants that were growing in the cold, those signs were visible only at 20 d.p.i. We could not observe any signs of photobleaching in Ler plants growing in the cold. The phenotype of plants in the cold was evaluated at four and then five weeks post-inoculation; although we could visualize differences in the penetrance of the silencing phenotype among the different accessions (number of inoculated plants that showed photobleaching symptoms), the number of plants affected by the silencing of $P D S$ for each accession was the same at four and five weeks. In addition, we did not observe any PDS silencing phenotype in one of the late flowering accessions (Tamm-2) (Figure 1).

2. VIGS can efficiently silence meiotic and post-meiotic genes in Arabidopsis.

The first gene selected to be knocked down in meiosis was $D M C 1$, because a successful knockdown showed univalents segregating at meiosis I and consequently high rates of pollen abortion that can be easily assessed through pollen phenotyping (Wijnker et al., 
2012). We cloned a 116 bp DMC1 cDNA sequence into the TRV2 vector in sense orientation (see table 4). Agrobacterium tumefaciens GV3101 was transformed with TRV2-DMC1 and used for agroinoculation of Col-0 seedlings of 21 d.p.g. Pollen abortion was observed in open flowers that were sampled at 26 d.p.i. Inoculated plants were left to set seed and displayed short siliques along the main stem, which resembles the sterility observed in $d m c 1$ mutants (Figure 2).

We then tested if VIGS could be used to silence other meiotic genes for which the mutant phenotypes also show aborted pollen and reduction in the seed-set manifested by the appearance of short siliques in inoculated plants. Primers were designed for PCR amplification of MLH1, SDS, PRD1, MTOPVIB and REC8, which were then cloned in TRV2 (see table 4). The fragments were cloned in antisense orientation for $M L H 1$, antisense for SDS, sense for PRD1, sense for MTOPVIB and antisense for REC8 (table 1 ). The silencing induced by the VIGS constructs lead to semi-sterility in inoculated Col0 plants (Figure 2). By counting the number of short siliques that appeared on each of the plants, we could verify the penetrance of the silencing phenotype caused by each of the VIGS constructs used (table 1). Using a t-test, only significant differences in the number of short siliques per plant were found for TRV2-SDS_1 (P-value $=0,00051309)$ TRV2-SDS_2 (P-Value $=0,00235684)$, TRV2-PRD1 ( $\mathrm{P}$-value $=0,0023793)$ and TRV2REC8 (P-value $=5,9952 \mathrm{E}-06$ ) when comparing inoculated plants with Col-0 controls (see materials and methods).
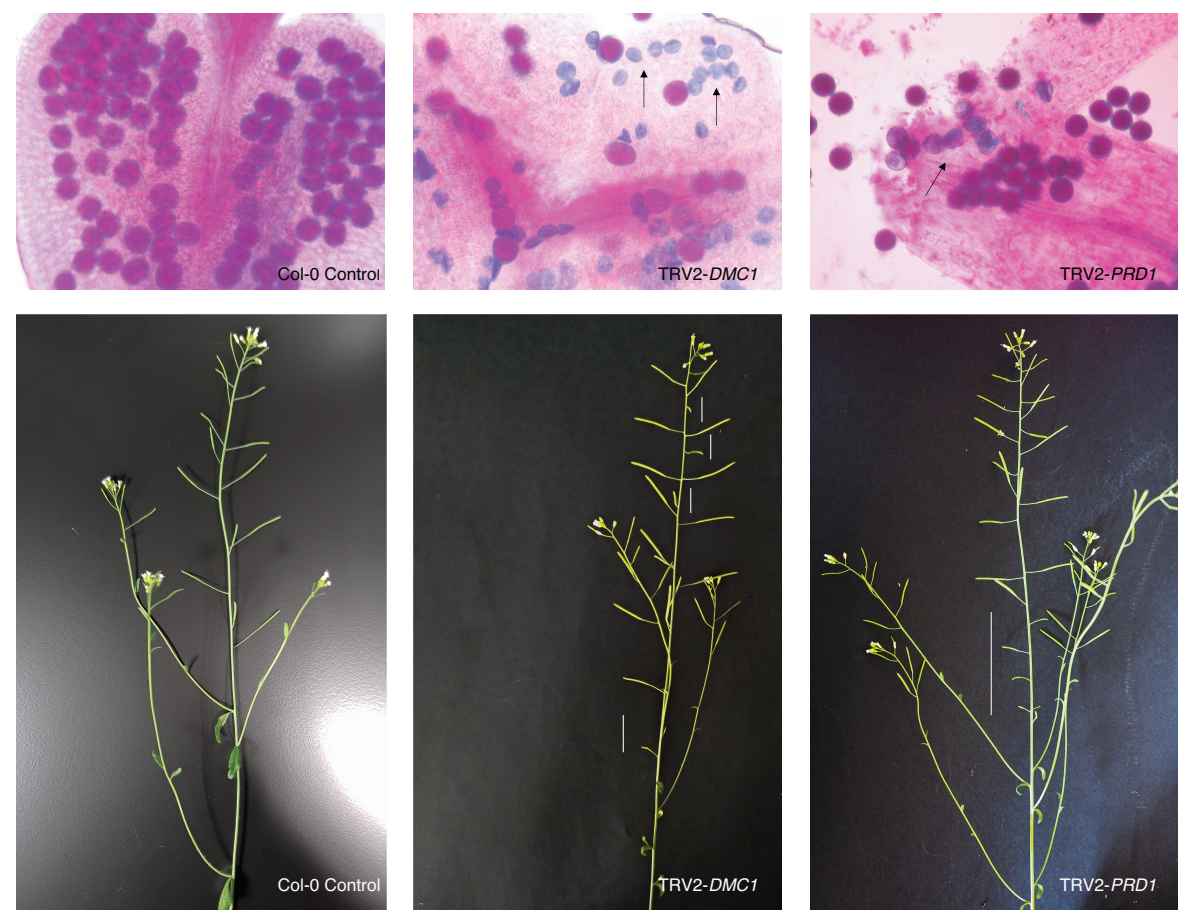
Figure 2. VIGS of $D M C 1$ and $P R D 1$ leads to semi-sterility in Arabidopsis plants. Upper row, from left to right (same magnification used): Col-0 Control plant depicts only regular pollen. Images of plants inoculated with TRV2-DMC1 and TRV-PRD1 show aborted pollen, indicated with the black arrows. Wildtype pollen observed in the pictures, likely correspond to cells not affected by the silencing. On the lower row from left to right: A control plant shows elongated siliques and no signs of sterility. Inoculated plants with TRV2-DMC1 and TRV2-PRD1 occasionally display short siliques likely caused by the silencing of $D M C 1 / P R D 1$. The regions where the short siliques appear in the plants are indicated with the white lines.

Table 1. Sterility assessed through the number of short (sterile) siliques in inoculated plants with VIGS vectors to target SDS, MLH1, PRD1, MTOPVIB, REC8. The orientation of the cloned insert, sense (s) or antisense (as) is indicated in each case. The number of inoculated plants displaying short siliques (\%) and the total number of short siliques per plant was used to infer the ability of the VIGS constructs to silence the target gene in Col-0 plants. Only the silencing of SDS, PRD1 and REC8 show significant differences $\left(^{*}\right)$ in the number of short siliques / plant compared to Col-0 wild-type controls.

\begin{tabular}{|c|c|l|}
\hline \multicolumn{3}{|c|}{ Penetrance of the silencing phenotype caused by VIGS in Col-0 } \\
\hline VIGS construct & $\begin{array}{c}\text { \% Plants displaying } \\
\text { short siliques }\end{array}$ & \multicolumn{1}{|c|}{ Number of short siliques / plant } \\
\hline Col-0 WT control & $45 \%(9 / 20)$ & $1,1,1,2,1,2,2,3,5$ \\
\hline TRV2-SDS_1 (as) & $80 \%(8 / 10)$ & $10,13,14,17,4,10,14,6$ \\
\hline TRV2-SDS_2 (as) & $56 \%(5 / 9)$ & $5,8,3,5,5$ \\
\hline TRV2-MLH1_1 (as) & $50 \%(4 / 8)$ & $2,2,2,3$ \\
\hline TRV2-MLH1_2 (as) & $83 \%(5 / 6)$ & $3,2,2,2,1$ \\
\hline TRV2-PRD1* (s) & $75 \%(9 / 12)$ & $9,5,8,5,2,3,4,4,7$ \\
\hline TRV2-MTOPVIB (s) & $58 \%(10 / 17)$ & $2,3,4,2,2,3,6,2,3,2$ \\
\hline TRV2-REC8* (as) & $81 \%(13 / 16)$ & $15,10,2,15,13,9,14,17,10,6,13,12,5$ \\
\hline
\end{tabular}

We also sought to develop a phenotypic marker that does not interfere with meiosis but would identify pollen affected by VIGS (see table 4). To this end, we designed VIGS constructs against $Q R T 1$ and QRT2. We created two VIGS vectors, TRV2-QRT1 and TRV2-QRT2, both cloned in antisense orientation (Table 4), and we evaluated if pollen tetrads appear in Col-0 and Ler inoculated plants. We then inoculated 21 d.p.g seedlings with either TRV2-QRT1 or TRV2-QRT2 and evaluated the phenotype 23 d.p.i (Table 2). We observed pollen in two flowers of each of 36 plants. We confirmed the formation of pollen tetrads in treated plants resembled the qtr mutant phenotype (Figure 3). Not all pollen in affected flowers formed pollen tetrads, thus producing mixtures of tetrads and regular and wild-type pollen (Figure 3 ). This suggests that not all the pollen grains produced are affected by the silencing. We also observed that TRV2-QRT2 could induce pollen tetrad formation in $100 \%$ of inoculated plants versus $70 \%$ of TRV2-QRT1 inoculated plants at the time point in which the phenotype was evaluated (table 2). 

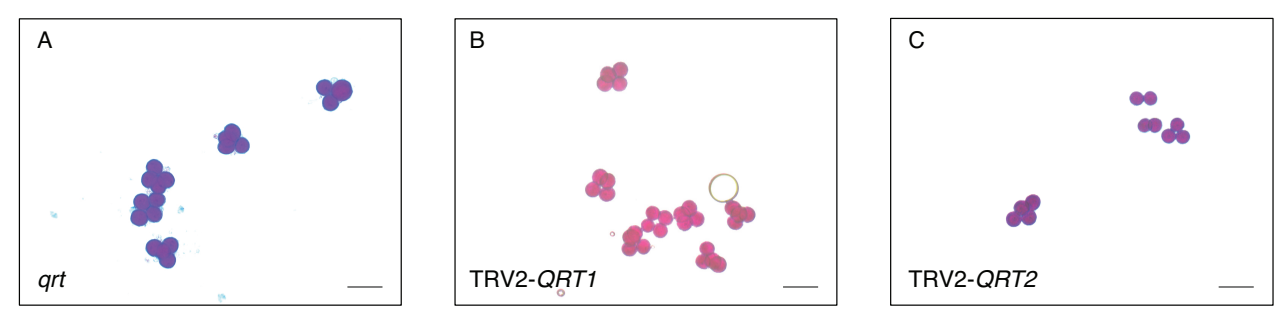

Figure 3. Pollen tetrads in a qrt mutant and $Q R T$ knockdowns, scale bar 50 um. Sample preparation was done as described in Peterson et al., 2010. A) shows pollen tetrads in homozygous qrt mutant while B) displays pollen tetrad produced by an inoculated plant with TRV2-QRT1. C) Shows one pollen tetrad among wild-type pollen due to the incomplete penetrance of TRV2-QRT2.

Table 2. Percentage of plants producing pollen tetrad after inoculation with TRV2-QRT1 and TRV2$Q R T 2$ in Col-0 and Ler accessions. The number of plants producing pollen tetrads over the total number of plants used for the sampling is given in parentheses.

\begin{tabular}{|c|c|c|}
\hline & TRV2-QRT1 & TRV2-QRT2 \\
\hline Col-0 & $55 \%(8 / 13)$ & $100 \%(12 / 12)$ \\
\hline Ler & $89 \%(5 / 6)$ & $100 \%(5 / 5)$ \\
\hline
\end{tabular}

3. VIGS to modify meiosis as a breeding tool: Production of polyploids.

After providing the proof of principle that VIGS can successfully be used to modify gene expression in meiosis of Arabidopsis, we decided to test the possible use of VIGS as a breeding tool by targeting OSD1. The osd 1 mutant phenotype is characterized by the production of unreduced (2n) gametes in both, male and female meiosis. A VIGS construct targeting OSD1 should allow one to produce tetraploid offspring when an inoculated plant is left to self-fertilize. One experiment then proves the possibility of VIGS targeting male and female meiosis, and simultaneously shows the feasibility of breeding with knockdown gametes. We constructed the TRV2-OSD1_1 and TRV2-OSD1_2VIGS constructs, using two different $250 \mathrm{bp}$ inserts cloned in antisense orientation (Table 4). We confirmed that both vectors induced the production of diploid pollen. In subsequent experiments only TRV2-OSD1_1 was used. To generate tetraploid offspring, we grew plants of the Arabidopsis accessions Est-1, Bor-4, Ler and Col-0. Plants were agroinoculated with TRV2-OSD1_1 and regularly checked after flowering to identify the moment in which larger pollen was formed (Figure 4). All preceding flowers were removed from the inflorescence, and the remaining flowers were left to self-fertilize. All siliques were later bulk-harvested for seeds. Because triploid (paternal excess) and tetraploid seeds are reportedly larger (Ravi, Marimuthu and Siddiqi, 2008) seeds were pre-selected based on seed size, and subsequently grown under standard conditions. Using flow cytometer to precisely assess ploidy level to identify diploids, triploids, and 
tetraploids, we confirmed the presence of triploids and tetraploids among the seedlings of the four accessions (see Figure 4). These results show that VIGS can be used to silence meiotic genes during female and male meiosis to generate polyploids in various Arabidopsis accessions.

A

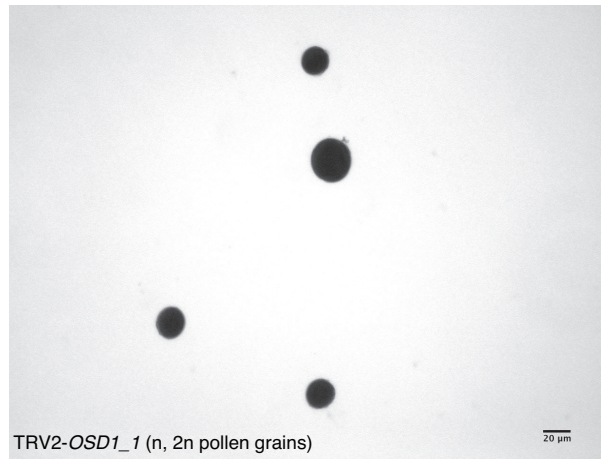

B
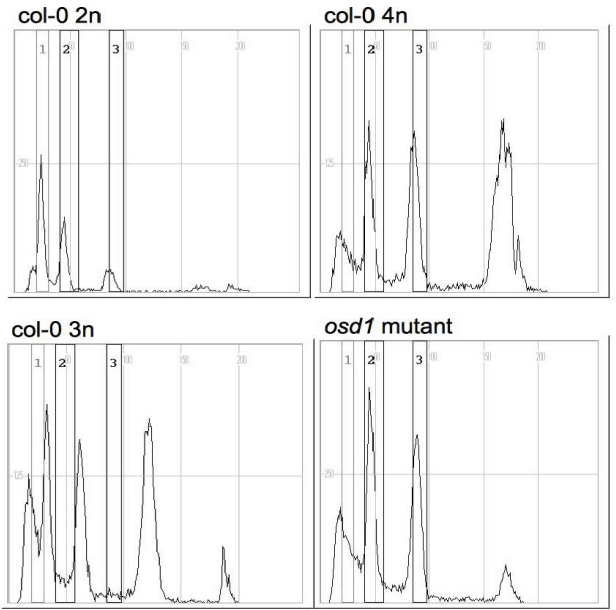

C

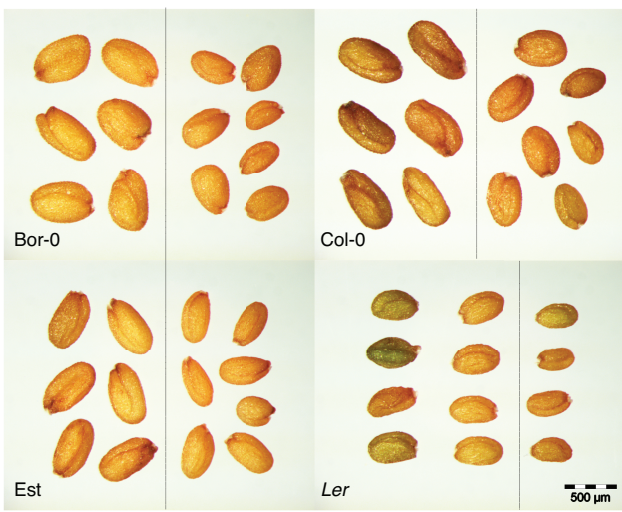


Figure 4: Generation of polyploid offspring by silencing OSD1. A) shows a pollen slide comparing diploid gametes (large) produced by a TRV2-OSD1 inoculated plant to wild-type haploid gametes (small), scale bar $20 \mathrm{um}$. The difference in size could be used as a proxy to select against wild-type gametes and verify the presence of unreduced or diploid gametes. B) Shows the histograms obtained from flow cytometry analysis of offspring derived from Col-0 TRV2-OSD1_1 inoculated plants. Diploid plants $(2 n=2 x)$, triploid plants $(2 n=3 x)$ and tetraploid plants $(2 n=4 x)$ were found in the offspring. Tetraploid plants obtained from an osd1 mutant were used as reference control. C) shows the differences in seed size found in the four accessions used in the experiment. For all of them, large seeds selected for ploidy analysis were identified and compared to diploid wild-type seeds. A line to separate big and small seeds is used in each of the pictures.

4. Characterization of REC8-PRD1-OSD1 simultaneous knockdown to induce a mitoticlike division during meiosis.

In the next experiments, we tested the method for simultaneously knocking down the three meiotic genes OSD1-PRD1-REC8, to induce a MiMe (Mitosis instead of Meiosis) phenotype, which would lead to the formation of unreduced (diploid) gametes carrying always one copy of each of the parental genomes (d'Erfurth et al., 2009). A gamete resulting from a mitotic-like division in combination with the genotype of a different female parent would give rise to a triploid, fully heterozygous offspring. To induce a MiMe phenotype, we decided to target OSD1-PRD1-REC8 simultaneously, using a TRV2 VIGS vector carrying an insert in sense orientation formed by three sequences homologous to CDS sequences of OSD1 (250 bp) PRD1 (267 bp) REC8 (200 bp). Each insert was previously tested independently in single TRV2 inoculation experiments (Figure 2 and 4, and Table 1). The experimental set-up consisted of Col-0 and F1 ColLer plants, used in two consecutive experiments. Seedlings at 21 and 28 d.p.g, respectively, were agroinoculated with TRV2-REC8-PRD1-OSD1, for which we intended to phenotype pollen in open flowers, three weeks p.i. However, such evaluation is complex, as both single, double or triple knockdowns can coexist in a single flower. Consequently, we observed in both, Col-0 and F1 plants that about 27 d.p.i (first batch) and 25 d.p.i (second batch), open flowers exhibited different phenotypes: production of 2n gametes, likely due to the silencing of OSD1, of OSD1-PRD1 or REC8-PRD1-OSD1. We also observed aborted pollen, that could be the result of silencing of either REC8, $P R D 1$ both of them simultaneously or REC8-OSD1 silencing (Figure 5B and 5D), already suggesting that the silencing of the three genes does not always happen simultaneously in the same flower and in the same meiocyte.

As affected flowers do not exhibit a clear pollen phenotype, the occurrence of triple knockdowns in at least some gametes was tested by analysing offspring. To this end we backcrossed plants with larger, putative $2 \mathrm{n}$ gametes to a male sterile Ler mutant (hereafter $m s$ ). Control crosses were done by crossing Col-0 to ms Ler. To confirm that a mitotic-like division took place in knocked down plants through the offspring, we first evaluated the offspring genotypes, of which we expected to find triploids. A total of 261 plants were genotyped for 40 markers using KASPAR probes, including five diploid F1 
controls plants (Figure 5). Marker dosage analysis was done to assess if a triploid background could be identified. In a total of six inoculated plants, we identified a marker dosage corresponding to triploid LLC - two copies of Ler and one copy of Col-0. We also identified one marker in one BC1 wild-type control that scored as LLC. Moreover, a total of four plants were identified as full diploid heterozygous and three as full homozygous diploid Ler, likely corresponding to contamination in crosses with wild-type Col-0 and Ler. To further confirm ploidy level through KASPAR marker dosage assessment, we decided to identify traits characteristic to triploids through the analysis of the phenotype on those plants. Despite some markers were scored as triploids, only one of the plants showed low pollen viability, but no other features related to triploid plants were observed, leading to the conclusion that triploids could not be recovered from these crosses.
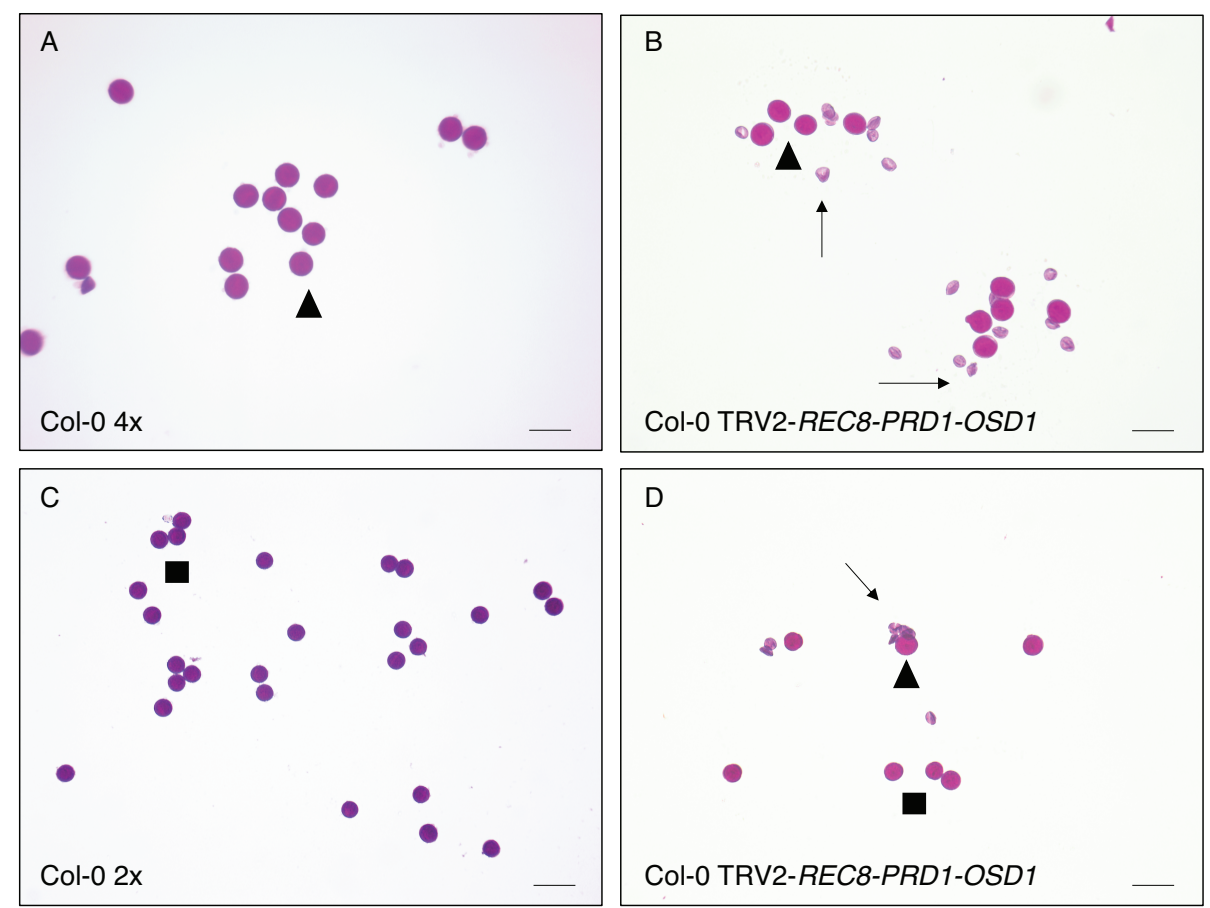

Figure 5. Pollen phenotype of $2 n=2 x$ and $2 n=4 x$ Col-0 control and TRV2-OSD1-PRD1-REC8 inoculated plants. Black arrowheads indicate enlarged pollen likely corresponding to diploid gametes. Black squares are placed next to haploid pollen, while the black arrows point at aborted pollen. A) shows large $2 n$ pollen grains from a tetraploid plant compared to $C$ ), which shows haploid pollen from a $2 \mathrm{n}$ Col0 control plant. B) and D) display the different pollen phenotypes observed in inoculated plants with TRV2-OSD1-PRD1-REC8, which is a miscellaneous of enlarged pollen, that could correspond to diploid cells, haploid-like pollen and aborted pollen. 


\section{Discussion.}

In the first part of our study, we analysed if different accessions of Arabidopsis thaliana are appropriate candidates for using VIGS in a transient silencing strategy. In a silencing assay of PDS the majority of them (26 of 28) showed symptoms after treatment, irrespective their flowering time. The results illustrate that silencing of the target gene is possible in several distinct genetic background, allowing in theory large scale experiments using different backgrounds which would be otherwise extremely lengthy through stable-transformation approaches (Burch-Smith 2004). However, the low penetrance of the silencing phenotype, (with less plants exhibited photobleaching symptoms in the late flowering accessions) is a matter of concern. The fact that plants inoculated with TRV2-PDS did not show high penetrance of the silencing phenotype (late flowering accessions) or no silencing phenotype of PDS (Ler) seems to indicate that the virus has difficulties to spread systemically when a cold treatment is applied, likely due to the inhibition of siRNAs produced (Szittya et al., 2003) and that it is unlikely that meiotic tissues will be ultimately affected.

Low proportions of plants affected by the silencing can be problematic when the intention is to knock down meiotic genes, as cold-treatment needed to induce flowering transition seems to conflict silencing. In such cases one might first vernalize the plants, before the agroinoculation with the desired VIGS construct. However, it has been reported that silencing efficiency in older plants is much lower than when seedlings at two-three leaf stage are inoculated (Burch-Smith et al., 2004; Burch-Smith, 2006; Becker, 2013; Bilichak and Kovalchuk, 2017). Another approach would be to inoculate plants in an early flowering flc or frigida background (Shindo et al., 2005). Additionally, older plants can be inoculated using the geminivirus Cabbage Leaf Curl Virus CbLCVbased VIGS system. VIGS using CbLCV allows to inoculate Arabidopsis plants about six weeks post germination using particle bombardment instead of agroinoculation (Turnage et al., 2002). In any case, the protocol should be adapted to late flowering accessions (i.e., developmental stage of the inoculation, test of different methods to deliver the virus) if meiotic genes have to be silenced.

Although VIGS has been used to target transgenes and endogenous genes in Arabidopsis before (Burch-Smith et al., 2004; Burch-Smith, 2006), our study has demonstrated an efficient silencing of meiotic and post-meiotic genes and could be applied to conduct fast genetic analyses of meiosis. We were able to accurately silence seven targets to induce the mutant phenotype. The phenotypes were easily evaluated through pollen phenotyping (aborted, unreduced pollen, and pollen tetrad formation), or through the presence of short siliques in case a semi-sterile phenotype was induced. Furthermore, the presence of short siliques indicates that female gametes are also affected by the silencing. This was later confirmed by the generation of tetraploid 
offspring in four Arabidopsis accessions, demonstrating that the method could be exploited to rapidly generate polyploids (Brownfield and Köhler, 2011).

Limitations of VIGS for simultaneous silencing of meiotic genes.

For the evaluation of simultaneous gene silencing in meiosis, we combined PRD1REC8-OSD1 target sequences in a single VIGS construct. This led to various phenotypes which seemed to correspond to a knockdown of each of the targets independently (i.e., pollen abortion, enlarged pollen) but not simultaneously. The fact that diploid gametes were visible among aborted pollen in some flowers could indicate that a knockdown of $O S D 1$ may have happened although no true polyploid were found in the offspring. We also hypothesized that enlarged pollen grains were derived from unbalanced chromosome segregation during meiosis, likely due to the silencing of only $P R D 1$. The observation of this complex phenotype suggests that simultaneous knockdowns of two or more genes using VIGS is not as straightforward as single ones, which was already suggested before (Becker, 2013). To account for these effects, we have considered different factors. First, the insert length seems to determine its stability within the viral genome (Lacomme, Hrubikova and Hein, 2003; Senthil-Kumar and Mysore, 2011). An optimum insert-length for efficient silencing in plants has been generally described to be about 200-350 bp (see review Senthil-Kumar and Mysore 2011), although this length seems to accept variations for VIGS systems used in different species (Becker, 2013; Senthil-Kumar and Mysore, 2014). In our experimental design we included different inserts ranging from 116 to 296 bp for inducing a phenotype of single knockdowns, and showed that fragments shorter than 200 bp can be effective in Arabidopsis. In contrast, very long inserts may be unstable or may limit the potential of the virus to spread from cell to cell (Becker and Lange, 2009; Pyott and Molnar, 2015). In this case, the virus may be able to remove such inserts through recombination, which ultimately leads to poor silencing efficiency (Burch-Smith et al., 2004; Senthil-Kumar and Mysore, 2011, 2014). As an example, Burch-Smith et al. 2004 reported that TRV2 insert length of $1.5 \mathrm{~kb}$ was still effective for VIGS in Nicotiana, while bigger inserts were not tolerated by the vector, possibly because an upper size limit of the insert was reached. The PRD1-REC8-OSD1 sequences used to create the insert summed up to $717 \mathrm{bp}$. In addition, our current VIGS vector contains a transposable element enlarging the size of the TRV2 vector with respect to the original virus (Liu et al., 2002) by $1 \mathrm{~kb}$, which makes the total length equal to about $11 \mathrm{~kb}$. It follows that inserts longer than approximately $250 \mathrm{bp}$ may not be tolerated by the virus, so the original vector described in Liu et al., 2002 should be retrieved and used in future assays if large inserts are to be tested.

A second factor that conditions insert stability, and hence effective silencing, is the orientation of the insert, as antisense orientation is generally accepted to be more efficient than sense orientation (Pflieger et al., 2008, 2014; Senthil-Kumar and Mysore, 2011; Bennypaul et al., 2012; Becker, 2013; Manmathan et al., 2013). In our set-up, a 
total of three single inserts were cloned in sense and nine in antisense. A total of 9 VIGS constructs induced silencing except for the ones targeting $M L H 1$ are cloned in antisense and MTOPVIB that is cloned in sense orientation (Table 4). The two single inserts REC8, $P R D 1$ were cloned in sense whereas OSD1 was cloned in antisense, leading in the three cases of silencing to the target gene and a strong phenotype (see Table 1). In the case of the triple VIGS construct TRV2-REC8-PRD1-OSD1, all the sequences were cloned in sense and two phenotypes were visible after inoculation: diploid gametes production and aborted pollen. Because we have observed silencing when inserts had been cloned in either sense or antisense, further investigation should follow. For instance, the penetrance of the silencing induced by a vector carrying the same insert sequence cloned in sense and antisense should be thoroughly assessed in each case to determine which one is more efficient. Thus, an adaptation of the VIGS vector paying special attention to insert characteristics (length and orientation) must be done in order to thoroughly induce and verify a complex meiotic phenotype.

To overcome the limitations of simultaneous endogenous silencing using VIGS, shorter lengths of individual inserts in sense and antisense orientation should be first tested alone and then in insert combination with other targets. Here, we have shown that $D M C 1$ was successfully knocked down using an insert length of $116 \mathrm{bp}$. Although other inserts should be further tested using lengths of about $100 \mathrm{bp}$, it already proves that efficient silencing in meiosis is possible. $100 \mathrm{bp}$-inserts will be an optimal length to be used in combination with other targets to induce double and triple simultaneous knockdowns, reaching a maximum length of stable inserts for TRV of $300 \mathrm{bp}$. Even shorter lengths can be considered: an insert length of 23 nt could already induce silencing if there is $100 \%$ homology to the target sequence (Burch-Smith et al., 2004). Also, cloning of short 40-60 base directed repeats has been reported to induce stronger phenotypes than vectors carrying long cDNA sequences (Lacomme, Hrubikova and Hein, 2003). 


\section{Materials and methods.}

1. Plant material and growing conditions.

The Arabidopsis thaliana accessions used in this study were selected from the collection of 350 natural accessions of Arabidopsis thaliana (see Table 1) (Li et al., 2010; Horton et al., 2012).

Table 3. List of "early flowering accessions" and "late flowering accessions" selected to be inoculated with TRV2-PDS.

\begin{tabular}{|c|c|}
\hline \multicolumn{2}{|c|}{ Late flowering accessions } \\
\hline Pna-17 & T540 \\
\hline UKNW06-460 & RRS-10 \\
\hline Lov-5 & Kas-2 \\
\hline Mr-0 & Goettingen-7 \\
\hline Ty-0 & Br-0 \\
\hline Tamm-2 & \\
\hline
\end{tabular}

\begin{tabular}{|c|c|}
\hline \multicolumn{2}{|c|}{ Early flowering accessions } \\
\hline HR5 & Da-0 \\
\hline Lom1-1 & Ha-0 \\
\hline Sha & Ep-0 \\
\hline Li-7 & Di-1 \\
\hline Fr-4 & Ler \\
\hline Bay-0 & Col-0 \\
\hline Li-3 & Mib-22 \\
\hline Pro-0 & Bor \\
\hline Mr-0 & Est \\
\hline Old-1 & \\
\hline
\end{tabular}

Seeds were sterilized using the hydrochloric fumigation procedure as described in Wijnker et al., 2014. After a stratification period of 1-5 days, seedlings were transferred to soil. Arabidopsis thaliana early flowering accessions used for all the experimental set- 
ups were grown in growth chambers, $21 \% 18^{\circ} \mathrm{C}, 16 \mathrm{H} / 8 \mathrm{H}$ light cycle and $60 \%-50 \% \mathrm{RH}$. Late flowering accessions were germinated in growth chambers, $21^{\circ} \mathrm{C} / 18^{\circ} \mathrm{C}, 16 \mathrm{H} / 8 \mathrm{H}$ light cycle and $60 \%-50 \% \mathrm{RH}$. Cold treatment was done in a cold room kept around $4^{\circ} \mathrm{C}$, $12 \mathrm{H} / 12 \mathrm{H}$ light cycle.

2. Vector construction and agrobacterium transformation.

Foreign inserts were designed from the CDS sequences of DMC1, OSD1, MLH1, PRD1, MTOPVIB, REC8, QRT1 and QRT2 using TAIR https://www.arabidopsis.og. TRV2-PDS and TRV2 empty vector as described in Burch-Smith et al., 2006 and can be found under stock number (CD3-1047) 4515440211, TRV2 under the stock number (CD3-1040) Tair accession 4515440204, TRV1 under the stock number (CD3-1039) Tair accession 5019327237.

Primers were designed to amplify CDS regions between 116 and 296 base pairs (see Table 4). Restriction sites were added to the primer sequences to allow cloning of the inserts into the TRV2 empty vector (see Table 4). Cloning of the individual inserts started with RNA extraction from Col-0 flower buds using Qiagen RNeasy Minikit (cat. $\left.n^{\circ} .74104\right)$. cDNA was synthesized from the extracted RNA using RevertAid First Strand CDNA Synthesis Kit (ThermoFisherScientific ${ }^{\mathrm{TM}}$, cat. $\mathrm{n}^{\circ} \mathrm{K} 1621$ ). The insert containing the three consecutives sequences for OSD1-PRD1-REC8 was presynthesized by Invitrogen (ThermoFisherScientific ${ }^{\top M}$ ) and delivered in a $\mathrm{PMK}-\mathrm{RQ}$ vector (kanamycine resistant) including the restriction sites Xhol and Smal. Amplified PCR products and $\mathrm{pMK}-\mathrm{RQ}::$ OSD1-PRD1-REC8 were digested using the FastDigest enzymes (ThermoFisherScientific TM) shown in Table 4. Ligation of the insert to the TRV2 backbone vector was done using a classical ligation reaction with T4 DNA ligase (ThermoFisherScientific ${ }^{\top M}$, cat. $n^{\circ}$. EL0014). The TRV2 plasmid was first cloned into E.coli using a classical heat shock as described in (Froger and Hall, 2007) and kanamycin as antibiotic of selection. The plasmid was extracted using Presto ${ }^{\mathrm{TM}}$ Mini Plasmid Kit (Genaid cat.n ${ }^{\circ}$. PDH100). We verified that the insert was successfully cloned into the TRV2 vector by sequencing using TRV2_SEQ primer (see Table 4). Upon sequencing verification, we transformed Agrobacterium tumefaciens GV3101 (pMP90) using gentamicin and kanamycin as antibiotic of selection. Positive colonies were confirmed by colony PCR and bacteria glycerol stocks were stored in a final $40 \%$ pure glycerol concentration. 
Table 4. Primer list used to amplify the cDNA regions of SDS, PRD1, MTOPVIB, MLH1, OSD1, QRT1, QRT2, DMC1 and REC8 in Arabidopsis thaliana, indicated in the $1^{\text {st }}$ column, together with the orientation of the insert: sense (s) or antisense (as). TRV2-PDS construct has been described in BurchSmith et al, 2006 For OSD1, SDS and MLH1, two constructs targeting different gene regions were designed. Forward primers (2nd column) and reverse primers (3rd column) are shown including the sequences of added restriction sites plus CA nucleotides at the beginning of the primer sequence. The primer used to sequence the VIGS vectors is TRV2-SEQ. Restriction sites for each primer pair (forward/reverse) are indicated in the $4^{\text {th }}$ column. The total size of the amplified fragment is indicated in the $5^{\text {th }}$ column. TRV2-SEQ primer was used to verify that the inserts were correctly cloned into the TRV2 vector.

\begin{tabular}{|c|c|c|c|c|}
\hline $\begin{array}{l}\text { insert } \\
\text { (gene) }\end{array}$ & fw_primer & rv_primer & $\begin{array}{l}\text { restriction } \\
\text { sites }\end{array}$ & $\begin{array}{l}\text { insert length } \\
\text { (bp) }\end{array}$ \\
\hline $\begin{array}{l}\text { OSD1_1 } \\
\text { (as) }\end{array}$ & CATCTAGACCCCAAGAAAGCTCTCTTCTC & CAGAATTCCCCAATATCTGGATTCACCGG & Xbal/EcoRl & 250 \\
\hline $\begin{array}{c}\text { OSD1_2 } \\
\text { (as) }\end{array}$ & CATCTAGAGGAGCTGGGACTGGAGGAGAC & CAGAATTCTGAGTAGCTTCTTCTCGGGTG & $X b a l / E c o R l$ & 250 \\
\hline $\begin{array}{l}\text { SDS_1 } \\
\text { (as) }\end{array}$ & CAGGATCCCGGAGGAGCTGATAACGAAG & CATCTAGAGCCAGAGTCGAAGAAGATGG & $\begin{array}{c}\text { BamHl / } \\
\text { Xbal }\end{array}$ & 265 \\
\hline $\begin{array}{l}\text { SDS_2 } \\
\text { (as) }\end{array}$ & CAGGATCCCCATCTTCTTCGACTCTGGC & CATCTAGATGGAGCAGTATGCCTTAGCA & $\begin{array}{c}\text { BamHI/ } \\
\text { Xbal }\end{array}$ & 267 \\
\hline $\begin{array}{l}\text { PRD1 } \\
\text { (s) }\end{array}$ & CATCTAGAGGTTTCCTTCAGTCATCCGA & CACTCGAGCAGTCTCCCCAAAAGATGGA & Xbal / Xhol & 267 \\
\hline $\begin{array}{l}\text { MTOPVIB } \\
\text { (s) }\end{array}$ & CATCTAGAGCAGCTATGTCCCTGACCTT & СACTCGAGGCTTCTTGGTCTTTCTGGGG & Xbal / Xhol & 202 \\
\hline $\begin{array}{c}\text { MLH1_1 } \\
\text { (as) }\end{array}$ & CACTCGAGTTGGCTCTGAAAGAGGAGGA & CAGAATTCTCAACATCATTTCCCAAGCA & Xhol / EcoRl & 239 \\
\hline $\begin{array}{c}\text { MLH1_2 } \\
\text { (as) }\end{array}$ & CACTCGAGAACCCAAAGGAAACTGCTGA & CAGAATTCTCCTCCTCTTTCAGAGCCAA & Xhol / EcoRl & 296 \\
\hline $\begin{array}{l}\text { QRT1 } \\
\text { (as) }\end{array}$ & CACCCGGGGAAACACCCTGAGAGGCAAA & CACTCGAGTGTCCGGTTGAAAAAGGATT & Smal / Xhol & 188 \\
\hline $\begin{array}{l}\text { QRT2 } \\
\text { (as) }\end{array}$ & CACCCGGGGCGGTACAAGTGAGCAATGT & CACTCGAGAACATTTCCTCGGGTGTCCA & Smal / Xhol & 198 \\
\hline $\begin{array}{l}\text { DMC1 } \\
\text { (s) }\end{array}$ & CAGGTACCTGCTGCAAAATGTCTGAGG & CACCCGGGCTAGTTTTTGCTGGCGGTCT & Kpnl / Smal & 116 \\
\hline $\begin{array}{c}\text { REC8 } \\
\text { (as) } \\
\text { TRV2_SEQ }\end{array}$ & GATGGACATTGTTACTCAAGGAAG & CACTCGAGTTACCTTGATGACTCCGCGA & Smal / Xhol & 200 \\
\hline
\end{tabular}




\section{Inoculation protocol.}

Agrobacterium incubation and inoculation procedure was done based on a modified protocol from Nimchuck et al., 2000. A single colony of each TRV2-insert and TRVRNA1 (hereafter TRV1) was inoculated in $2 \mathrm{ml} \mathrm{YEP} \mathrm{medium} \mathrm{using} 70 \mu \mathrm{g} / \mathrm{ml}$ gentamicin and $70 \mu \mathrm{g} / \mathrm{ml}$ kanamycin. The pre-cultures were incubated at $28^{\circ} \mathrm{C}$, overnight, shaking at $170 \mathrm{rpm}$. A $3 \mathrm{ml}$ YEP medium using $70 \mu \mathrm{g} / \mathrm{ml}$ gentamicin and $70 \mu \mathrm{g} / \mathrm{ml}$ kanamycin were inoculated with $150 \mathrm{ul}$ of the precultures and incubated for $13 \mathrm{H}$ maximum, $28^{\circ} \mathrm{C}$ shaking at $170 \mathrm{rpm}$. After $13 \mathrm{H}$, cultures were centrifuged at $5000 \mathrm{rpm}, \mathrm{RT}$. The supernatant was discarded and the pellet resuspended in $3 \mathrm{ml}$ incubation medium containing $10,5 \mathrm{~g} \mathrm{~K} 2 \mathrm{HPO}, 4,5 \mathrm{~g} \mathrm{KH} 2 \mathrm{PO} 4,1 \mathrm{~g}\left(\mathrm{NH}_{4}\right)_{2} \mathrm{SO}_{4}, 0,5 \mathrm{~g}$ Sodium citrate, $1 \mathrm{mM}$ $\mathrm{MgSO}_{4}, 1 \mathrm{~g}$ Glucose, $1 \mathrm{~g}$ Fructose, $4 \mathrm{ml}$ Glycerol, 1,95g MES and adjust the $\mathrm{pH}$ to 5,6 using $\mathrm{HCL}$ and sterilize by filtration using a $22 \mu \mathrm{m}$ filter pore, plus $50 \mathrm{ug} / \mathrm{ml}$ of acetosyringone per $1 \mathrm{~L}$ of ddH2O. The cultures were incubated for $5-7 \mathrm{H}, 23^{\circ} \mathrm{C}$ and shaking at $170 \mathrm{rpm}$. After the incubation time, they were centrifuged, $5000 \mathrm{rpm}, \mathrm{RT}$ and resuspended in infiltration medium containing 1,95g MES and 2,04g MS per $1 \mathrm{~L}$ of ddH2O. $\mathrm{OD}_{600}$ for each culture was adjusted to 0,4 and each TRV2 was mixed together with TRV1 in a 1:1 ratio to be leaf-infiltrated using a $20 \mathrm{ml}$ needless syringe.

4. Statistical analysis.

A t-test (alpha $=0.05)$ was done comparing each sub-set of plants inoculated with TRV2 to the subset of Col-0 WT subset of plants, based on the number of short siliques/plant (Table 1). Plants that did not show any short silique were not included in the analysis. Only significant differences in the number of short/siliques per plant were found for the comparisons between Col-0 wild-type to with TRV2-SDS_1, TRV2-SDS_2, TRV2-PRD1 and TRV2-REC8.

Table 5. Results from the statistical the t-test analysis (Alpha=0.05). Comparison between the number of short siliques / plant in Col-0 WT sub-set and VIGS sub-sets to target SDS, MLH1, PRD1, MTOPVIB and $R E C 8$. The obtained $p$-values indicate that there are significant differences $\left({ }^{*}\right)$ between Col- 0 wildtype plants and inoculated plants with TRV2-SDS_1, TRV2-SDS_2, TRV2-PRD1 and TRV2-REC8.

\begin{tabular}{|c|c|}
\hline $\mathbf{N}^{\circ}$ Short siliques/plant in Col-0 and VIGS plants & T-test (P-value) \\
\hline Col-0 WT- TRV2-SDS1 ${ }^{*}$ & 0,00051309 \\
\hline Col-0 WT- TRV2-SDS-2 ${ }^{*}$ & 0,00235684 \\
\hline Col-0 WT - TRV2-MLH1_1 & 0,6315715 \\
\hline Col-0 WT- TRV2-MLH1_2 & 1 \\
\hline Col-0 WT - TRV2 - PRD1 ${ }^{*}$ & 0,0023793 \\
\hline Col-0 WT - TRV2-MTOPVIB & 0,15195705 \\
\hline Col- WT - TRV2-REC8 ${ }^{*}$ & $5,9952 \mathrm{E}-06$ \\
\hline
\end{tabular}




\section{Phenotypic evaluation and offspring generation.}

To stain pollen grains, we use the Alexander staining solution as described in Peterson et al., 2010.

The knockdown of $D M C 1$ and $P R D 1$ was validated by checking flowers systematically three weeks post-inoculation. The qrt phenotype was verified by sampling open flowers at a single time point.

The generation of polyploid offspring upon silencing of OSD1 was done using plants of the four different accessions, in which the silencing was obvious, through selffertilization. Using a binocular, we could easily identify bigger seeds (Ravi, Marimuthu and Siddiqi, 2008) which were used to predict the ploidy level. Offspring from REC8PRD1-OSD1 presumably knocked down flowers was generated by carefully removing one anther of all the open flowers in treated plants to verify that large pollen was produced. Only the flowers producing large pollen, and not only aborted pollen, were used to pollinate a single inflorescence of $m s$ Ler. The crosses were sampled individually and all the plants deriving from the same cross were always grown and genotyped in separate clusters. The genotyping data set can be retrieved from http://doi.org/10.4121/uuid:99b33f26-8d74-42ac-bf51-7a5c1e5b490c.

\section{References.}

1. Azumi, Y. et al. (2002) 'Homolog interaction during meiotic prophase I in Arabidopsis requires the SOLO DANCERS gene encoding a novel cyclin-like protein', The EMBO Journal. Oxford, UK: Oxford University Press, 21(12), pp. 3081-3095. doi: 10.1093/emboj/cdf285.

2. Baulcombe, D. (2004) 'RNA silencing in plants', Nature., 431, p. 356. Available at: http://dx.doi.org/10.1038/nature02874.

3. Becker, A. (2013) Virus-Induced Gene Silencing. Methods in Molecular Biology. Edited by A. Becker. Humana Press. doi: 10.1007/978-1-62703-278-0.

4. Becker, A. and Lange, M. (2009) 'VIGS - genomics goes functional', Trends in Plant Science, 15(1), pp. 1-4. doi: 10.1016/j.tplants.2009.09.002.

5. Bennypaul, H. S. et al. (2012) 'Virus-induced gene silencing (VIGS) of genes expressed in root, leaf, and meiotic tissues of wheat.', Functional and Integrative Genomics, 12(1), pp. 143-156. doi: 10.1007/s10142-011-0245-0.

6. Bilichak, A. and Kovalchuk, I. (2017) 'Increasing a stable transformation efficiency of Arabidopsis by manipulating the endogenous gene expression using virus-induced gene silencing.', in Kovalchuk, I. (ed.) Plant epigenetics. Methods in Molecular Biology. Boston, MA: Humana Press, pp. 225-236. doi: 10.1007/978-3-319-55520-1.

7. Blary, A. et al. (2018) 'FANCM liimits meiotic crossovers in Brassica crops', Frontiers in Plant Science, 9(368), pp. 1-13. doi: 10.3389/fpls.2018.00368.

8. Brigneti, G. et al. (2004) 'Virus-induced gene silencing in Solanum species.', The Plant Journal, 39(2), pp. 264-272. doi: 10.1111/j.1365-313X.2004.02122.x.

9. Brownfield, L. and Köhler, C. (2011) 'Unreduced gamete formation in plants: Mechanisms and prospects', Journal of Experimental Botany, 62(5), pp. 1659-1668. doi: 10.1093/jxb/erq371. 
10. Burch-Smith, T. M. et al. (2004) 'Applications and advantages of virus-induced gene silencing for gene function studies in plants.', Plant Journal, 39(5), pp. 734-746. doi: 10.1111/j.1365-313X.2004.02158.x.

11. Burch-Smith, T. M. et al. (2006) 'Efficient virus-linduced gene silencing in Arabidopsis', Plant Physiology, 142(1), pp. 21-27. doi: 10.1104/pp.106.084624.

12. Copenhaver, G. P., Keith, K. C. and Preuss, D. (2000) 'Tetrad analysis in higher plants. A budding technology.', Plant Physiology, 124(1), pp. 7-16. doi: 10.1104/pp.124.1.7.

13. Couteau, F. et al. (1999) 'Random chromosome segregation without meiotic arrest in both male and female meiocytes of a dmc1 mutant of Arabidopsis.', The Plant Cell, 11(9), p. 1623-1634 doi: 10.2307/3871042.

14. Crismani, W. et al. (2012) 'FANCM limits meiotic crossovers', Science, 336(6088), pp. 15881590. doi: $10.1126 /$ science. 1220381.

15. Cromer, L. et al. (2012) 'OSD1 promotes meiotic progression via APC/C inhibition and forms a regulatory network with TDM and CYCA1;2/TAM', PLoS Genetics, 8(7), pp. 1-14. doi: 10.1371/journal.pgen.1002865.

16. d'Erfurth, I. et al. (2010) 'The CYCLIN-A CYCA1;2/TAM is required for the meiosis I to meiosis II transition and cooperates with OSD1 for the prophase to first meiotic division transition', PLoS Genetics, 6(6), pp. 1-12. doi: 10.1371/journal.pgen.1000989.

17. D'Erfurth, et al. (2009) 'Turning meiosis into mitosis.', PLoS Biology, 7(6), p. e1000124. doi: 10.1371/journal.pbio.1000124.

18. D'Erfurth, I. et al. (2008) 'Mutations in AtPS1 (Arabidopsis thaliana Parallel Spindle 1) lead to the production of diploid pollen grains.', PLoS Genetics, 4(11), pp. 1-9. doi: 10.1371/journal.pgen.1000274.

19. Dion, É. et al. (2007) 'An Arabidopsis MLH1 mutant exhibits reproductive defects and reveals a dual role for this gene in mitotic recombination', Plant Journal, 51(3), pp. 431-440. doi: 10.1111/j.1365-313X.2007.03145.x.

20. Dirks, R. et al. (2009) 'Reverse breeding: A novel breeding approach based on engineered meiosis', Plant Biotechnology Journal,7(9) pp. 837-845. doi: 10.1111/j.14677652.2009.00450.x.

21. Fernandes, J. B. et al. (2017) 'Unleashing meiotic crossovers in hybrid plants', Proceedings of the National Academy of Sciences, 115(10), pp. 2431-2436. doi: 10.1073/pnas.1713078114.

22. Francis, K. E., Lam, S. Y. and Copenhaver, G. P. (2006) 'Separation of Arabidopsis pollen tetrads Is regulated by QUARTET1, a Pectin Methylesterase Gene', Plant Physiology, 142(3), pp. 1004-1013. doi: 10.1104/pp.106.085274.

23. Froger, A. and Hall, J. E. (2007) 'Transformation of Plasmid DNA into E. coli using the heat shock method', Journal of Visualized Experiments, (6). doi: 10.3791/253.

24. Giorno, F. et al. (2013) 'Ensuring reproduction at high temperatures: The heat stress response during anther and pollen development', Plants, 2(3), pp. 489-506. doi: 10.3390/plants2030489.

25. Hand, M. L. and Koltunow, A. M. G. (2014) 'The genetic control of apomixis: Asexual seed formation', Genetics, 197(2), pp. 441-450. doi: 10.1534/genetics.114.163105.

26. Horton, M. W. et al. (2012) 'Genome-wide patterns of genetic variation in worldwide Arabidopsis thaliana accessions from the RegMap panel', Nature Genetics, 44(2), pp. 212216. doi: 10.1038/ng.1042.

27. Lacomme, C., Hrubikova, K. and Hein, I. (2003) 'Enhancement of virus-induced gene silencing through viral-based production of inverted-repeats', The Plant Journal, 34(4), pp. 543-553. doi: 10.1046/j.1365-313X.2003.01733.x. 
28. Lambing, C., Franklin, F. C. H. and Wang, C.-J. R. (2017) 'Understanding and manipulating meiotic recombination in plants.', Plant Physiology, 173(3), pp. 1530-1542. doi: 10.1104/pp.16.01530.

29. Li, Y. et al. (2010) 'Association mapping of local climate-sensitive quantitative trait loci in Arabidopsis thaliana', Proceedings of the National Academy of Sciences, 107(49), pp. 21199-21204. doi: 10.1073/pnas.1007431107.

30. Liu, Y. et al. (2002) 'Tobacco Rar1, EDS1 and NPR1/NIM1 like genes are required for Nmediated resistance to Tobacco mosaic virus', Plant Journal, 30(4), pp. 415-429. doi: 10.1046/j.1365-313X.2002.01297.x.

31. Manmathan, H. et al. (2013) 'Virus-induced gene silencing of Arabidopsis thaliana gene homologues in wheat identifies genes conferring improved drought tolerance', Journal of Experimental Botany, 64(5), pp. 1381-1392. doi: 10.1093/jxb/ert003.

32. Mieulet, D. et al. (2016) 'Turning rice meiosis into mitosis', Cell Research. 26(11), pp. 12421254. doi: $10.1038 / \mathrm{cr} .2016 .117$.

33. Mieulet, D. et al. (2018) 'Unleashing meiotic crossovers in crops', Nature plants ., 4(12), pp 1010-1016. doi 10.1038/s41477-018-0311-x

34. Minorsky, P. V. (2003) 'The Hot and the Classic.', Plant Physiology, 133(2), pp. 441-442. doi: 10.1104/pp.900032.A.

35. De Muyt, A. et al. (2007) 'AtPRD1 is required for meiotic double strand break formation in Arabidopsis thaliana.', EMBO Journal, 26(18), pp. 4126-4137. doi: 10.1038/sj.emboj.7601815.

36. Nimchuk, Z. et al. (2000) 'Eukaryotic fatty acylation drives plasma membrane targeting and enhances function of several type III effector proteins from Pseudomonas syringae', Cell, 101(4), pp. 353-363. doi: 10.1016/S0092-8674(00)80846-6.

37. Peterson, R., Slovin, J. P. and Chen, C. (2010) 'A simplified method for differential staining of aborted and non-aborted pollen grains', International Journal of Plant Biology, 1(2), pp. 66-69. doi: 10.4081/pb.2010.e13.

38. Pflieger, S. et al. (2008) 'Efficient virus-induced gene silencing in Arabidopsis using a "onestep" TYMV-derived vector', Plant Journal, 56(4), pp. 678-690. doi: 10.1111/j.1365313X.2008.03620.x.

39. Pflieger, S. et al. (2014) 'The "one-step" Bean pod mottle virus (BPMV)-derived vector is a functional genomics tool for efficient overexpression of heterologous protein, virus-induced gene silencing and genetic mapping of BPMV R-gene in common bean (Phaseolus vulgaris L.)', BMC Plant Biology, 14(232), pp. 1471-2229. doi: 10.1186/s12870-014-0232-4.

40. Preuss, D., Rhee, S. Y. and Davis, R. W. (1994) 'Tetrad analysis possible in Arabidopsis with mutation of the QUARTET (QRT) genes.', Science, 264(5164), pp. 1458-1460. doi: 10.1126/science.8197459.

41. Pyott, D. E. and Molnar, A. (2015) 'Going mobile: Non-cell-autonomous small RNAs shape the genetic landscape of plants', Plant Biotechnology Journal, 13(3), pp. 306-318. doi: 10.1111/pbi.12353.

42. Ratcliff, F., Martin-Hernandez, A. M. and Baulcombe, D. C. (2001) 'Tobacco rattle virus as a vector for analysis of gene function by silencing', Plant Journal, 25(2), pp. 237-245. doi: 10.1046/j.0960-7412.2000.00942.x.

43. Ravi, M., Marimuthu, M. P. A. and Siddiqi, I. (2008) 'Gamete formation without meiosis in Arabidopsis', Nature, 451(7182), pp. 1121-1124. doi: 10.1038/nature06557.

44. Rhee, S. Y. and Somerville, C. R. (1998) 'Tetrad pollen formation in quartet mutants of Arabidopsis thaliana is associated with persistence of pectic polysaccharides of the pollen mother cell wall', The Plant Journal, 15(1), pp. 79-88. doi: 10.1046/j.1365- 
313X.1998.00183.x.

45. Ruiz, M. T., Voinnet, O. and Baulcombe, D. C. (1998) 'Initiation and maintenance of virusinduced gene silencing.', The Plant Cell, 10(6), pp. 937-946. PMC144041

46. Senthil-Kumar, M. and Mysore, K. S. (2011) 'New dimensions for VIGS in plant functional genomics', Trends in Plant Science. Elsevier Ltd, 16(12), pp. 656-665. doi: 10.1016/j.tplants.2011.08.006.

47. Senthil-Kumar, M. and Mysore, K. S. (2014) 'Tobacco rattle virus-based virus-induced gene silencing in Nicotiana benthamiana', Nature Protocols, 9(7), pp. 1549-1562. doi: 10.1038/nprot.2014.092.

48. Shindo, C. et al. (2005) 'Role of FRIGIDA and FLOWERING LOCUS C in determining variation in flowering time of Arabidopsis', Plant Physiology, 138(June), pp. 1163-1173. doi: 10.1104/pp.105.061309.1.

49. De Storme, N. et al. (2013) 'Volume-based pollen size analysis: An advanced method to assess somatic and gametophytic ploidy in flowering plants', Plant Reproduction, 26(2), pp. 65-81. doi: 10.1007/s00497-012-0209-0.

50. Szittya, G. et al. (2003) 'Low temperature inhibits RNA silencing-mediated defence by the control of siRNA generation', EMBO Journal, 22(3), pp. 633-640. doi: 10.1093/emboj/cdg74.

51. Turnage, M. A. et al. (2002) 'Geminivirus-based vectors for gene silencing in Arabidopsis', The Plant Journal, 30(1), pp. 107-114. doi: 10.1046/j.1365-313X.2002.01261.x.

52. Unver, T. and Budak, H. (2009) 'Virus-induced gene silencing, A post transcriptional gene silencing method.', International Journal of Plant Genomics, pp. 1-8. doi: $10.1155 / 2009 / 198680$.

53. Vrielynck, N. et al. (2016) 'A DNA topoisomerase VI - like complex initiates meiotic recombination', Science, 351(6276), pp. 939-944.

54. Wang, K. et al. (2012) 'The role of rice HEI10 in the formation of meiotic crossovers', PLoS Genetics, 8(7), pp. 1-13. doi: 10.1371/journal.pgen.1002809.

55. Wijnker, E. et al. (2012) 'Reverse breeding in Arabidopsis thaliana generates homozygous parental lines from a heterozygous plant', Nature Genetics, 44(4), pp. 467-470. doi: 10.1038/ng.2203.

56. Wijnker, E. et al. (2014) 'Hybrid recreation by reverse breeding in Arabidopsis thaliana', Nature Protocols, 9(4), pp. 761-772. doi: 10.1038/nprot.2014.049. 
To the start of the fight.

\author{
"This chapter is about war: \\ The one fought by viruses in order to ensure their replication, \\ and by the host cell in its defense of its statuos quo \\ and the restriction of infection by an intruding agent. \\ The outcome is not always certain, and depends on the balance of actions \\ and counteractions between host defense systems \\ and the virus scape mechanisms." \\ Gustavo Fermin and Paula Tennant.
}




\section{Chapter 3}

\section{Efficient reverse breeding by VIGS-mediated transient crossover reduction.}

\section{Authors.}

Vanesa Calvo-Baltanas ${ }^{1}$, Cris L. Wijnen ${ }^{1}$, Nina Lukhovitskaya2§, C. Bastiaan de Snoo³, Linus Hohenwarter ${ }^{4}$, Hans de Jong ${ }^{1}$, Arp Schnittger ${ }^{4}$ and Erik Wijnker ${ }^{1 *}$.

\section{Author affiliations.}

1 Laboratory of Genetics, Wageningen University \& Research, P.O. Box 166700 AA Wageningen, The Netherlands.

2) Institut de Biologie Moléculaire des Plantes, Centre National de la Recherche Scientifique, UPR 2357, Université de Strasbourg, 67084 Strasbourg, France.

3) Rijk Zwaan R\&D Fijnaart, Eerste Kruisweg 9, 4793 RS Fijnaart, the Netherlands.

4) Department of Developmental Biology, University of Hamburg, Institute for plant sciences and microbiology, 22609 Hamburg, Germany.

$\S$ Current address: Division of Virology, Department of Pathology, University of Cambridge, Tennis Court Rd, Cambridge, CB2 1QP, UK. 


\begin{abstract}
.
F1 heterozygotes are traditionally generated by crossing homozygous parental lines. The opposite is achieved through reverse breeding, in which parental lines are generated from a heterozygote. Reverse breeding can be used to develop new F1 hybrid varieties without having prior access to homozygous breeding lines. For successful reverse breeding, the heterozygotes' homologous chromosomes must be divided over two haploid complements, which is achieved by suppression of meiotic crossover (CO) recombination. We here show two innovations that facilitate efficient reverse breeding. Firstly, we demonstrate that downregulation of $\mathrm{CO}$ rates can be accomplished using virus-induced gene silencing (VIGS). We obtain transgene-free parental lines for a heterozygote in just two generations. Secondly, we show that incomplete $\mathrm{CO}$ suppression opens up several alternative strategies for the preservation of hybrid phenotypes through reverse breeding.
\end{abstract}

\title{
Main text.
}

Heterozygous F1 hybrids are among the highest producing crop varieties (Schnable and Springer, 2013) and result from intercrossing homozygous parental lines. Existing hybrids are usually further improved through the introgression of new alleles into their parental lines. In an alternative approach, large numbers of new and potentially better heterozygous genotypes could be generated in outcrossing populations, for example by intercrossing different commercially available heterotic hybrids and selecting the best performing heterozygotes in their offspring. However, this potential is rarely, if ever exploited, because unique heterozygotes selected from outcrossing populations cannot be maintained: when they set seed, their unique allele combinations are lost through meiotic recombination. This restriction can be overcome by reverse breeding, in which new parental lines for any heterozygote can be post-hoc generated from the selected heterozygote itself(Dirks et al., 2009; Wijnker et al., 2012) (Fig. 1). By obtaining its parental lines, a heterozygote can be recreated as F1 hybrid. 


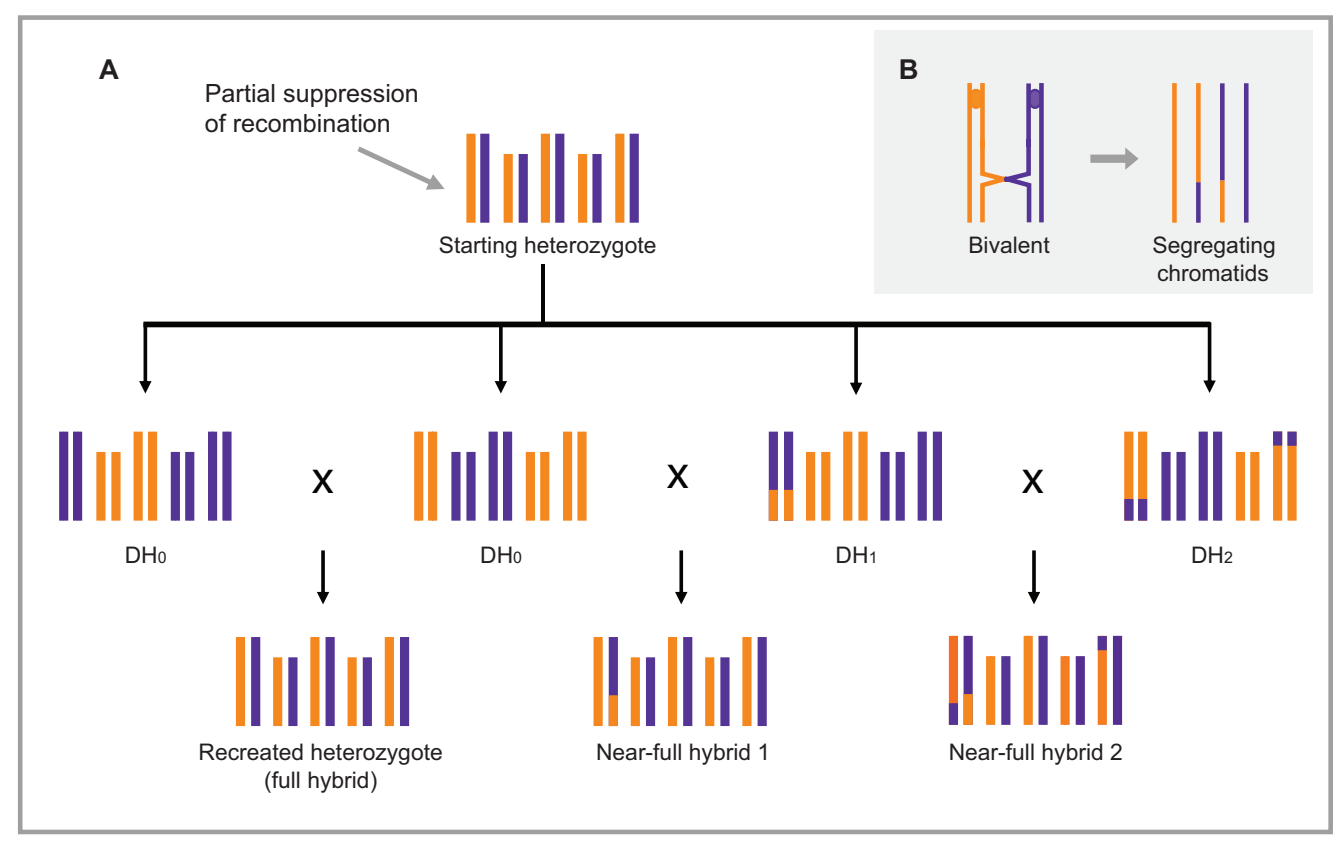

Figure 1. Reverse breeding (heterozygote reconstruction) and near-full hybrid generation through partial crossover (CO) suppression. Panel A: a starting Arabidopsis heterozygote (top), for which parental lines are to be made. Five chromosome pairs are shown, with homologs in orange and purple. Meiotic COs are partially suppressed in this heterozygote, resulting in $\mathrm{DH}$ offspring as shown in the middle row having 0,1 or $2 \mathrm{COs}\left(\mathrm{DH}_{0}, \mathrm{DH}_{1}\right.$ and $\mathrm{DH}_{2}$ respectively). Intercrossing complementing $\mathrm{DH}_{0}$ reconstitutes the heterozygote as a full hybrid (left), which is similar to the approach by Wijnker et al., 2012(Wijnker et al., 2012). Intercrossing $\mathrm{DH}_{0}$ with $\mathrm{DH}_{1}$ (middle) or $\mathrm{DH}_{1}$ with $\mathrm{DH}_{2}$ (right) generates near-full hybrids, which have small homozygous genomic regions. Note for example that in the cross of $\mathrm{DH}_{1}$ with $\mathrm{DH}_{2}$ chromosome 1 is largely heterozygous, since the parental lines complement one another in the distal chromosome region. Panel B: Recombinant but also non-recombinant chromatids can segregate in the presence of $\mathrm{CO}$. Detail of a bivalent pair with one meiotic $\mathrm{CO}$ is shown (left). Only two of the four chromatids are recombinant (right).

A proof of concept study (Wijnker et al., 2012) showed the feasibility of reverse breeding in an Arabidopsis thaliana hybrid. This was achieved by the complete knockdown of meiotic $\mathrm{CO}$ formation in a F1 hybrid using a dominantly acting RNAi transgene targeting the essential meiotic recombinase DISRUPTED MEIOTIC CDNA 1 (DMC1). Without COs, non-recombinant chromosomes segregate to gametes. These gametes were regenerated as haploid plants, and self-fertilized to give rise to homozygous diploid lines (doubled haploids; DH) from which complementing parental lines were selected and crossed to reconstitute the starting heterozygote (Fig. 1A). In short, reverse breeding requires the consecutive suppression of recombination and the conversion of resulting gametes to $\mathrm{DH}$ offspring. 
Since the translation of this technique to crops may be challenging, we here set out to overcome two major drawbacks of the original approach. Firstly, the use of a transgene to suppress $\mathrm{CO}$ formation in a heterozygote is impractical. Stable transformation of a selected heterozygote can be complex and a transgene that dominantly compromises fertility renders half of the offspring (the genotypes carrying the construct) useless for further breeding. We asked if virus-induced gene silencing (VIGS) could be used to transiently suppress meiotic $\mathrm{CO}$ formation in a hybrid(Ratcliff, Martin-Hernandez and Baulcombe, 2001; Burch-Smith et al., 2004, 2006) and whether gametes resulting from VIGS-modified meiosis can be used to generate offspring of desired genotypic composition.

Secondly, CO formation is indispensable for chromosome segregation in plants. Without COs, homologs segregate randomly (as univalents) at anaphase I. This causes aneuploidy in gametes and semi-sterility. Viable haploid gametes can still be formed in the absence of $\mathrm{CO}$, when the homologues of each chromosome pair by random chance segregate to opposite poles(Wijnker et al., 2012). The probability of regular disjunction is a function of the chromosome number of the plant(Dirks et al.,

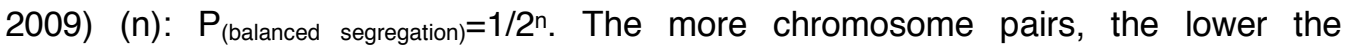
probability of viable gamete formation, and the lower the chance of obtaining parental lines. In Arabidopsis about $1 / 2^{5}=3 \%$ of meiotic events generates viable spores in the absence of COs.

The suppression of $\mathrm{CO}$ formation enriches for the segregation of non-recombinant chromosomes to gametes, but complete $\mathrm{CO}$ suppression is not essential. Gametes carrying exclusively non-recombinant chromosomes will occasionally be formed in wild-type meiosis (see Fig. 1B) although they are usually rare, especially when the chromosome number is high (see Supplementary tables 1-6). A reduction of $\mathrm{CO}$, rather than complete $\mathrm{CO}$ suppression, might present a favorable intermediate approach to enrich for viable gametes carrying only or mostly, non-recombinant chromosomes(Dirks et al., 2009). The presence of parallel pathways that lead to CO formation in plants allows theoretically for fine-tuning $\mathrm{CO}$ rates(Lambing, Franklin and Wang, 2017). Mutants of MUTS HOMOLOGUE5 (MSH5) show about $87 \%$ reduction in COs in Arabidopsis(Higgins et al., 2008) and we therefore targeted MSH5 using a VIGS construct to reduce $\mathrm{CO}$ formation.

The efficiency of VIGS to downregulate $M S H 5$ was assessed by inoculating plants at the five-leaf stage with a VIGS vector (TRV2-AtMSH5) and evaluating meiotic progression. MSH5 knocked-down plants exhibited high levels of aborted pollen about three weeks after inoculation and siliques that failed to elongate (Supplementary fig. 1), consistent with a msh5 mutant phenotype(Lu et al., 2008). Chromosome spreads of late meiotic cell complements confirmed the mis-segregation of chromosomes during meiosis (Supplementary fig. 1). Reduced fertility was typically observed for three to 
four days on flowers, after which the plants reverted to a wild-type phenotype, exhibiting viable pollen and long siliques.

To evaluate the feasibility of breeding with gametes resulting from VIGS-mediated reduction of recombination, we inoculated an $\mathrm{F} 1$ (Landsberg erecta $\mathrm{x}$ Columbia) with TRV2-AtMSH5. Once the flowers showed a high fraction of aborted pollen, they were crossed to GFP-tailswap, a haploid inducer line for Arabidopsis(Ravi and Chan, 2010). Haploid offspring were obtained and self-fertilized to give rise to $111 \mathrm{DH}$ offspring that were genotyped for 42 markers evenly spaced over the genome (Supplementary fig. 2).

Among the 111 offspring we identified $24 \mathrm{DHs}$ (20 different genotypes) that carry only non-recombinant chromosomes (Supplementary file 1). These lines, which are also known as chromosome substitution lines (CSLs), are henceforth referred to as $\mathrm{DH}_{0}$. The population is significantly enriched for $\mathrm{DH}$ lines originating from nonrecombinant chromosomes in comparison to a previously published wild-type population (Fig. 2) (Kolmogorov-Smirnov test; $a=0.01$ ). Among these $20 \mathrm{DH}_{0}$ genotypes we identified six complementing parental pairs that, when crossed, recreate the starting hybrid (Supplementary file 1). All DH offspring developed normally and were fully fertile. This shows that VIGS can transiently modify meiotic recombination in wild-type hybrids. This approach strongly increases reverse breeding efficiency and speed as compared to an earlier approach in which CO suppression resulted from using a dominant RNAi transgene(Wijnker et al., 2012, 2014). Firstly, using VIGS, all recovered offspring are transgene-free and fertile, while in the previous set-up half of the offspring were transgenic and sterile implying a two-fold increase of efficiency. Moreover, without stable transformation a heterozygous genotype can be recreated as $\mathrm{F} 1$ in three generations where previously six generations were required. 

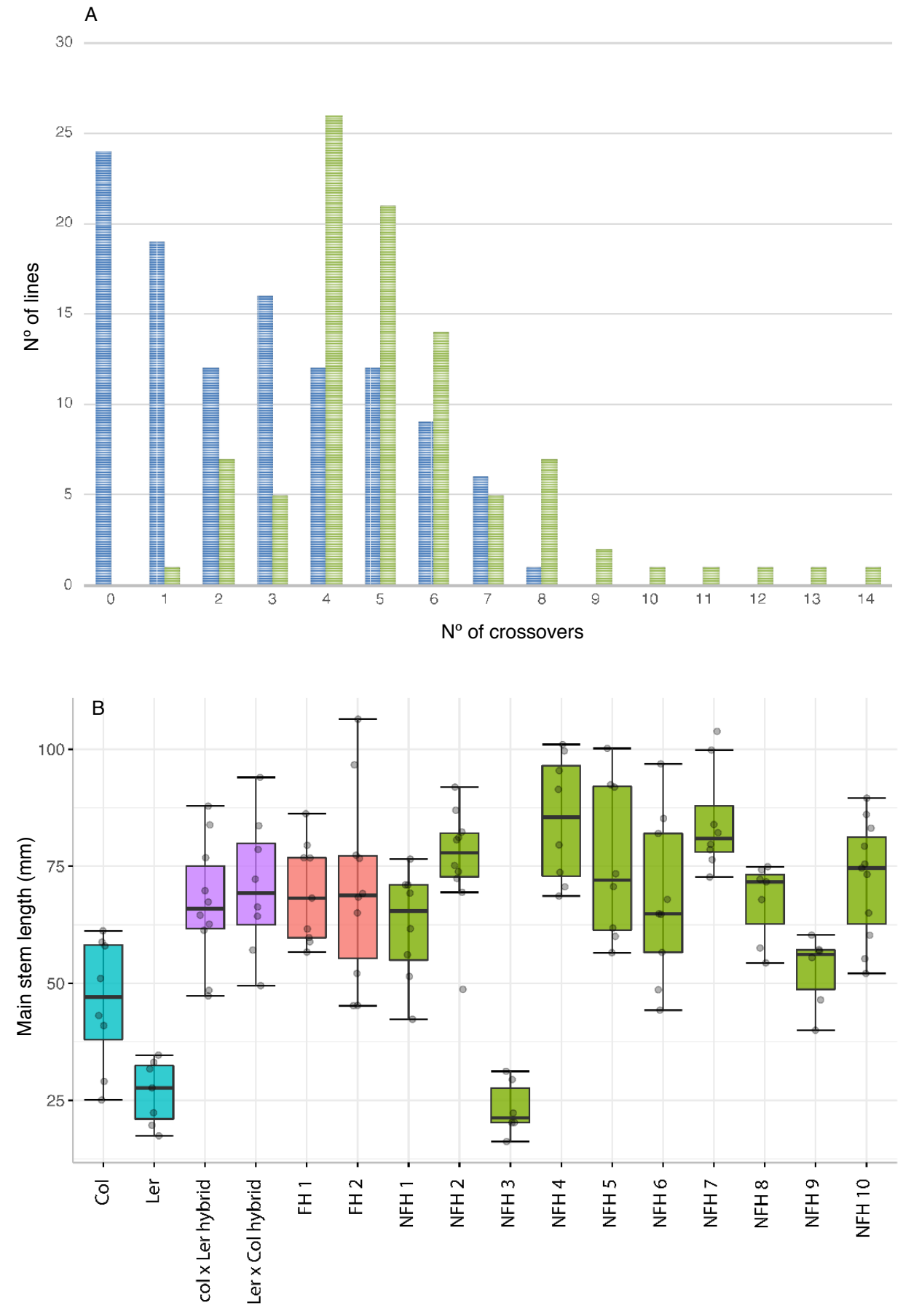
Figure 2. Crossover (CO) distributions in reverse breeding- and wild-type $\mathrm{DH}$ offspring (top panel) and comparison of hybrid phenotypes (lower panel). Top panel (A) shows the observed CO number for wild-type DH offspring (in green) and for reverse breeding DH offspring (in blue). Note that reverse breeding are enriched for $\mathrm{DH}$ offspring having 0 and $1 \mathrm{CO}$. Lower panel (B) shows box-and-whisker plots for main stem length at the moment of flowering of parental lines, full hybrids ( $\mathrm{FH}$ ) and near-full hybrids (NFH). Parental lines Col-0 and Ler (shown in blue), Col-0 x Ler reciprocal hybrids (violet), full hybrids (coral) and near-full hybrids (green). Genotypes of near full hybrids are presented in the supplementary file.

For the exact recreation of a heterozygous genotype, complementing $\mathrm{DH}_{0}$ are required, but in practice the recreation of the hybrid phenotype will be the ultimate goal. The use of a $\mathrm{DH}_{1}$ (i.e. a $\mathrm{DH}$ with one recombinant chromosome) in a cross to recreate a hybrid, leads to a decrease of heterozygosity (hereafter $\mathrm{DOH}$ ) in the reconstituted hybrid distal to the CO position (Fig. 1). We hypothesized that only in case $\mathrm{DOH}$ negatively affects the hybrid phenotype, it is of concern for reverse breeding. In our offspring we identified $19 \mathrm{DH}_{1}$ and $12 \mathrm{DH}_{2}$ with one and two COs per genome respectively, with the remainder of $58 \mathrm{DH}$ having three to eight COs, which is in the range of wild-type meiosis and likely result from incomplete penetrance of VIGS (Supplementary file 1). Depending on $\mathrm{CO}$ positions in the $\mathrm{DH}_{1}$ and $\mathrm{DH}_{2}$ offspring, we noted the possibility of identifying four additional parental pairs in which a near-full hybrid would show a DOH less than $2.5 \%$ of the total genome length. Seven parental pairs would give rise to near-full hybrids in which DOH is less than $5 \%$. Only near-full hybrids with one $\mathrm{CO}$ show less than $2.5 \% \mathrm{DOH}$. In one parental pair $\left(\mathrm{DH}_{1}\right.$ line $44 \mathrm{x}$ $\mathrm{DH}_{2}$ line 41) $\mathrm{COs}$ on the same chromosome arm partly compensate, similar to the $\mathrm{DH}_{1}$ $\mathrm{x} \mathrm{DH}_{2}$ cross illustrated in Fig. 1, generating a near-full hybrid with a $\mathrm{DOH}$ of $4.2 \%$.

The phenotypic impact of $\mathrm{DOH}$ can be explored experimentally. We therefore intercrossed $\mathrm{DH}$ lines to create near-full hybrids with increasing levels of $\mathrm{DOH}$ ranging from $1.28 \%-32.1 \%$ (Supplementary fig. 3). These were grown together with the starting heterozygote and full hybrids (recreated heterozygotes) and compared standard growth parameters: flowering time, main stem length, rosette diameter and dry weight at flowering time. No significant differences were found between the starting hybrid and the full hybrids (one-way ANOVA; FT p-value $=0.3015 ;$ MSL $p$-value = 0.9347; RD p-value $=0.8655$; DW p-value $=0.2697$; Fig. 2B; Supplementary fig. 3 ). Also, no significant differences between the full hybrid and the near-full hybrids were found, with the exception of one: a near-full hybrid that has a similar short stem length as one of its parental lines (Fig. 2), which is likely caused by homozygosity of the main effect erecta locus that is homozygous in this specific hybrid(Stinchcombe et al., 2009).

These results mainly illustrate that $\mathrm{DOH}$ not necessarily negatively impacts hybrid performance. It is possible to estimate the expected $\mathrm{DOH}$ in near-full hybrids resulting from a single $\mathrm{CO}$. Arabidopsis has five linkage groups (chromosomes). One CO recombines one linkage group $\left(1 / 5^{\text {th }}\right)$ and this $C O$ exchanges anything between zero and half of the linkage group, which averages at $1 / 4^{\text {th }}$ of the linkage group (typically 
half a chromosome arm). Expected $\mathrm{DOH}$ caused by a single $\mathrm{CO}$ thus equals on average $\left(1 / 4^{*} 1 / 5=\right) 5 \%$ of the total linkage map length. Of the ten near-full hybrids (with one $\mathrm{CO}$ ) that we can produce, five have a $\mathrm{DOH}$ less than $5 \%$ in Mbp, exactly as predicted as the Arabidopsis genetic map correlates well with the physical chromosome length. The more chromosomes a species has, the lower the relative $\mathrm{DOH}$ resulting from one $\mathrm{CO}$. In a species with ten chromosome pairs (e.g. maize) one $\mathrm{CO}$ causes a $\mathrm{DOH}$ of $2.5 \%$. This decreases even further when, as in many species, $\mathrm{COs}$ locate relatively distal on chromosomes. Under such a scenario, not only $\mathrm{DH}_{0}$, but also $\mathrm{DH}_{1}$ and $\mathrm{DH}_{2}$ may prove worthy parental lines, provided that resulting near-full hybrids are phenotyped to assess their performance.

Our experiments show that $\mathrm{CO}$ formation during meiosis can be adjusted to favorable levels, by targeting $M S H 5$ rather than $D M C 1$ as was previously done. The lower the $\mathrm{CO}$ number, the more $\mathrm{DH}_{0}$ and $\mathrm{DH}_{1}$ occur in the offspring (Fig. 2, Supplementary tables 1-6), but also the higher the level of gamete abortion. Depending on ones' interest in obtaining $\mathrm{DH}_{0}\left(\right.$ and $\left.\mathrm{DH}_{1}\right)$, the optimal $\mathrm{CO}$ rate can be calculated to balance one against the other. Especially in species with higher chromosome numbers, such considerations matter. In a species with ten chromosome pairs, in which a typical bivalent has two $\mathrm{COs}$, complete $\mathrm{CO}$ suppression generates $100 \% \mathrm{DH}_{0}$ offspring but results in just $0.10 \%$ of spore viability. Reducing COs by $75 \%$ from 20 to five COs per meiosis- would increase spore viability 32 fold to $3 \%$, since then only five rather than ten univalent pairs segregate. Of those offspring, $5.6 \%$ are $\mathrm{DH}_{0}$ (supplementary table 3 ). This is low in comparison to complete $\mathrm{CO}$ suppression, but it is a substantial 60.000 fold increase in comparison to wild-type meiosis. Likewise, chances for obtaining $\mathrm{DH}_{1}$ in its offspring are $18.8 \%$, equal to about 10.000 fold increase. Supplementary tables 1-6 give expected $\mathrm{DH}_{0}$ and $\mathrm{DH}_{1}$ numbers at different levels of $\mathrm{CO}$ suppression and different chromosome numbers. Such calculations will help to determine the best possible approach for other species than Arabidopsis.

Apart from generating parental lines for heterozygotes, reverse breeding provides a way to generate populations of $\mathrm{DH}_{0}$, also known as chromosome substitution lines(Wijnker et al., 2012). Due to the low number of segregating loci (chromosomes), such populations are near unparalleled tools to identify QTL and map complex epistatic interactions (Wijnen et al., 2018) . Since in mixed $\mathrm{DH}_{0} / \mathrm{DH}_{1}$ populations the number of segregating loci remains near minimal, the detection power of QTLs and epistasis is unlikely to decrease much. Efficient reverse breeding strategies are therefore a way towards detection and mapping complex interactions. VIGS vectors are available for a multitude of crops(Senthil-Kumar and Mysore, 2011) and exploring these for the modification of meiosis may advance breeding strategies in other species. The recent identification of meiotic mutants may present further attractive targets for VIGS-mediated breeding strategies. 
Acknowledgements: This research was supported by the Netherlands the Organization for Scientific Research (NWO) through number STW-14389 (E.W.) and the European Community $(E C)$ though the Marie-Curie Initial Training Network "COMREC", project 606956 funded under FP7-PEOPLE (V.C.-B.). We thank Cilia Lelivelt (Rijk Zwaan, Fijnaart, Netherlands) for her support in processing genotyping samples and Bas Zwaan (Wageningen University, Netherlands) for moments of reflection during our research. We thank Laurens Deurhof (Wageningen University, Netherlands) and Shinichiro Komaki (NAIST, Japan) for their support and help during the experimental set-up.

Author contributions: E.W. conceptualized the research; E.W., A.S. and H.D.J. were involved in supervision and funding acquisition. V.C.B. and E.W. planned research, performed crosses. N.L. helped with setting up VIGS experiments and construct design; Cloning and VIGS experiments were done by V.C.B.; L.H. performed cytogenetic analyses with help of E.W.; C.B.d.S. performed genotyping; V.C.B., E.W. and C.L.W. designed, performed and analyzed the phenotyping experiment. V.C.B. and E.W. processed and interpreted experimental data, designed the figures and drafted the manuscript with the help of A.S. and H.d.J. All authors discussed the results and commented on the manuscript.

Competing interests: Rijk Zwaan B.V. holds a patent for reverse breeding. C.B.d.S. is a current employee of Rijk Zwaan and E.W. is a former employee. H.d.J. previously received research funding from Rijk Zwaan.

\section{Online Methods.}

Plant material and growth. Arabidopsis thaliana plants used in crosses and for VIGS inoculation were grown in potting soil in growth chambers (Percival), $21 \% 18^{\circ} \mathrm{C}$, $16 \mathrm{H} / 8 \mathrm{H}$ light cycle and $60 \%-50 \%$ relative humidity. Haploid offspring were grown under similar conditions in a greenhouse. For phenotyping, seeds of $\mathrm{DH}$ offspring, reconstituted full hybrids and near-full hybrids were vernalized by sowing on wet filter paper and placed them for several days in the dark at $4^{\circ} \mathrm{C}$ for four days to ensure uniform germination. Plants were grown on $4 \times 4 \mathrm{~cm}$ Rockwool blocks and watered with a flooding system with a Hyponex nutrient solution three times per week in a randomized block design with five blocks and two replicates per genotype in each block. Climate chamber conditions were set to $16 \mathrm{~h} / 8 \mathrm{~h}$ and $20 / 18^{\circ} \mathrm{C}$ day/night cycle, light was set to $125 \mu \mathrm{molm}-2 \mathrm{~s}-1$ and there was $70 \%$ relative humidity.

Plasmid construction and Agrobacterium inoculation. Two MSH5 cDNA regions were amplified using primers to which BamH (forward) and Xbal (reverse) restriction sites were added. The MSH5_F1/R1 and MSH5_F2/R2 primer pairs give fragments of $242 \mathrm{bp}$ and $254 \mathrm{bp}$ respectively, and were used to generate the TRV2-AtMSH5 and TRV2-AtMSH5_2 constructs. Both PCR products were introduced individually into the vector TRV2 (pYL156)(Liu et al., 2002) following a classical digestion-ligation reaction. 
After sequence verification, the TRV2-AtMSH5 and TRV2-AtMSH5_2 vectors were transformed into Agrobacterium tumefaciens GV3101 (pMP90) strain. The incubation and inoculation protocol was executed as described in Nimchuk et al., 2000(Nimchuk et al., 2000). Plant agroinoculation was done by leaf-infiltration(Vaghchhipawala et al., 2011) of TRV2-AtMSH5 in combination with TRV1 (pYL192)(Liu et al., 2002) or TRV2AtPDS in combination with PTRV1 in a 1:1 ratio. TRV2-AtMSH5 and TRV2-AtMSH5_2 induced similar pollen phenotypes in inoculated plants, after which only TRV2-AtMSH5 was used for further experiments.

Primers used:

MSH5_F1 5'- CAGGATCCAAGCCATCGATCATTTACGC -3'

MSH5_R1 5'- CATCTAGAACTTGGACTTCACTGCCCAC -3'

MSH5_F2 5' - CAGGATCCAAGCCATCGATCATTTACGC-3'

MSH5_R2 5' - CATCTAGAACTTGGACTTCACTGCCCAC -3'

Selection of TRV-AtMSH5 agroinoculated plants for pollen phenotyping. A total of 109 plants were inoculated with TRV2-AtMSH5 in three consecutive experiments $(52+42+15)$. Three non-inoculated plants were grown as negative controls in every batch as well as three to four plants in each batch that were inoculated with TRV2AtPDS to silence PDS as positive control (Burch-Smith et al., 2006). To evaluate a successful knock-down of $\mathrm{MSH}$, we assessed pollen viability in flowers that opened three weeks post-inoculation and the two consecutive weeks. One anther was removed from each flower and placed on a slide with a drop of a modified Alexander stain(Peterson, Slovin and Chen, 2010) to observe pollen viability. Pollen on control plants remained viable throughout the test periods. The number of affected flowers was not consistent. Within an inflorescence, flowers with high levels of pollen abortion usually appear consecutively, and a semi-sterile phenotype was present for about six consecutive days after the first sterile flowers appeared.

$\mathrm{DH}$ production. To produce doubled haploids, F1 hybrid plants of Ler x Col-0 plants were inoculated with TRV2-AtMSH5 as described above. Once flowers appeared, pollen of flowers displaying high levels of dead pollen were crossed to the inducer line GFP-tailswap (Ravi and Chan, 2010). Of the three consecutively grown batches 27, 19 and 15 plants were used. Other plants did not show a semi-sterile phenotype. From these plants we used 132, 77 and 60 flowers for pollination of GFPtailswap. Haploid selection was done as described in Wijnker et al., 2014 (Wijnker et al., 2014). Among the 369 offspring we identified 113 haploid offspring. For 111 of these we obtained $\mathrm{DH}$ seeds.

Phenotypical analysis of (near-)full hybrids. At the moment of flowering, flowering time (FT) was recorded and main stem length (MSL), rosette diameter (RD) and dry weight (DW) were measured for each plant. Phenotypic data was corrected for spatial trends and block effects with the SpATS R package, and the resulting spatial corrected 
raw data was used for further analyses. To establish whether the intercrosses of the $\mathrm{DHO}$ resulted in different full hybrids, these were compared with the parental wild-type F1 using one-way ANOVA.

To assess the performance of the NFH in comparison with the $\mathrm{FH}$, a Dunnett test was conducted in which line $\mathrm{FH} 2$ was used as a control line.

Cytology. F1 hybrid flower buds were sampled 18 days post-inoculation. The inflorescences were incubated in Carnoy: a 3:1 mix of glacial acetic acid (HAc) and $99,8 \% \mathrm{EtOH}$ and kept overnight at $4{ }^{\circ} \mathrm{C}$. inflorescences were then washed twice with $70 \% \mathrm{EtOH}$ (in water) and stored at $4^{\circ} \mathrm{C}$. Meiotic chromosome spreads were made as previously described in Ross et al. (1996)(Ross, Fransz and Jones, 1996), stained with DAPI and analyzed using a Zeiss microscope equipped with epifluorescence optics.

Calculations of expected frequencies of $\mathrm{DH}_{0}$ and $\mathrm{DH}_{1}$. To calculate the expected number of $\mathrm{DH}_{0}$ and $\mathrm{DH}_{1}$ in Supplementary tables 1-6, the expected number of nonrecombinant and recombinant chromatids was first determined for one chromosome (i.e. the case in which the haploid chromosome number equals 1 ). If $a$ is the number of COs per bivalent, then the chance of recovering a non-recombinant chromatid in a spore, and hence the chance of recovering a $\mathrm{DH}_{0}$, equals $\mathrm{P}_{(\mathrm{DHO})}=(1 / 2)$. The chance of finding a chromatid with one $\mathrm{CO}$ (and hence recovering a $\mathrm{DH}_{1}$ ) equals $\mathrm{P}_{(\mathrm{DH} 1)}=\mathrm{a}(1 / 2) \mathrm{a}$. For higher haploid chromosome numbers $(n)$, the expected number of nonrecombinant chromatids equal $P_{(\mathrm{DH})}=(1 / 2)$ an and $P_{(\mathrm{DH} 1)}=a n(1 / 2)$ an For $\mathrm{CO}$ numbers 1 , $P_{(D H O)}=1-a / 2$ and $P_{(D H 1)}=a / 2$. For higher haploid chromosome numbers $P_{(D H O)}=(1-a / 2)^{n}$ and $P_{(D H 1)}=n a / 2(1-\alpha / 2)^{(n-1)}$.

\section{References and notes.}

1. Burch-Smith, T. M. et al. (2004) 'Applications and advantages of virus-induced gene silencing for gene function studies in plants.', Plant Journal, 39(5), pp. 734-746. doi: 10.1111/j.1365-313X.2004.02158.x.

2. Burch-Smith, T. M. et al. (2006) 'Efficient virus-linduced gene silencing in Arabidopsis', Plant Physiology, 142(1), pp. 21-27. doi: 10.1104/pp.106.084624.

3. Dirks, R. et al. (2009) 'Reverse breeding: A novel breeding approach based on engineered meiosis', Plant Biotechnology Journal, 7 (9) pp. 837-845. doi: 10.1111/j.14677652.2009.00450.x.

4. Higgins, J. D. et al. (2008) 'AtMSH5 partners AtMSH4 in the class I meiotic crossover pathway in Arabidopsis thaliana, but is not required for synapsis', The Plant Journal, 55(1), pp. 28-39. doi: 10.1111/j.1365-313X.2008.03470.x.

5. Lambing, C., Franklin, F. C. H. and Wang, C.-J. R. (2017) 'Understanding and manipulating meiotic recombination in plants.', Plant Physiology, 173(3), pp. 1530-1542. doi: 10.1104/pp.16.01530.

6. Liu, Y. et al. (2002) 'Tobacco Rar1, EDS1 and NPR1/NIM1 like genes are required for Nmediated resistance to tobacco mosaic virus', Plant Journal, 30(4), pp. 415-429. doi: 10.1046/j.1365-313X.2002.01297.x.

7. Lu, X. et al. (2008) 'The Arabidopsis MutS homolog AtMSH5 is required for normal meiosis', Cell Research, 18(5), pp. 589-599. doi: 10.1038/cr.2008.44. 
8. Nimchuk, Z. et al. (2000) 'Eukaryotic fatty acylation drives plasma membrane targeting and enhances function of several type III effector proteins from Pseudomonas syringae', Cell, 101(4), pp. 353-363. doi: 10.1016/S0092-8674(00)80846-6.

9. Peterson, R., Slovin, J. P. and Chen, C. (2010) 'A simplified method for differential staining of aborted and non-aborted pollen grains', International Journal of Plant Biology, 1(2), pp. 66-69. doi: 10.4081/pb.2010.e13.

10. Ratcliff, F., Martin-Hernandez, A. M. and Baulcombe, D. C. (2001) 'Tobacco rattle virus as a vector for analysis of gene function by silencing', The Plant Journal, 25(2), pp. 237-245. doi: 10.1046/j.0960-7412.2000.00942.x.

11. Ravi, M. and Chan, S. W. L. (2010) 'Haploid plants produced by centromere-mediated genome elimination', Nature., 464(7288), pp. 615-618. doi: 10.1038/nature08842.

12. Ross, K. J., Fransz, P. and Jones, G. H. (1996) 'A light microscopic atlas of meiosis in Arabidopsis thaliana.', Chromosome Research, 4(7), pp. 507-516. doi: 10.1007/BF02261778.

13. Schnable, P. S. and Springer, N. M. (2013) 'Progress toward understanding heterosis in crop plants', Annual Review of Plant Biology, 64(1), pp. 71-88. doi: 10.1146/annurev-arplant042110-103827.

14. Senthil-Kumar, M. and Mysore, K. S. (2011) 'New dimensions for VIGS in plant functional genomics', Trends in Plant Science. Elsevier Ltd, 16(12), pp. 656-665. doi: 10.1016/j.tplants.2011.08.006.

15. Stinchcombe, J. R. et al. (2009) 'Polymorphic genes of major effect: Consequences for variation, selection and evolution in Arabidopsis thaliana', Genetics, 182(3), pp. 911-922. doi: 10.1534/genetics.108.097030.

16. Vaghchhipawala, Z. et al. (2011) 'Agroinoculation and Agroinfiltration: Simple tools for complex gene function analyses.', in A., P. (ed.) Plant reverse genetics. Methods in Molecular Biology. Humana Press, Totowa, NJ, pp. 65-76. doi: 10.1007/978-1-60761-682-5.

17. Wijnen, C. L. et al. (2018) 'A complete chromosome substitution mapping panel reveals genome-wide epistasis in Arabidopsis', bioRxiv. Available at: http://biorxiv.org/content/early/2018/10/05/436154.abstract.

18. Wijnker, E. et al. (2012) 'Reverse breeding in Arabidopsis thaliana generates homozygous parental lines from a heterozygous plant', Nature Genetics, 44(4), pp. 467-470. doi: 10.1038/ng.2203.

19. Wijnker, E. et al. (2014) 'Hybrid recreation by reverse breeding in Arabidopsis thaliana', Nature Protocols, 9(4), pp. 761-772. doi: 10.1038/nprot.2014.049. 


\section{Supplementary Figures and Tables.}
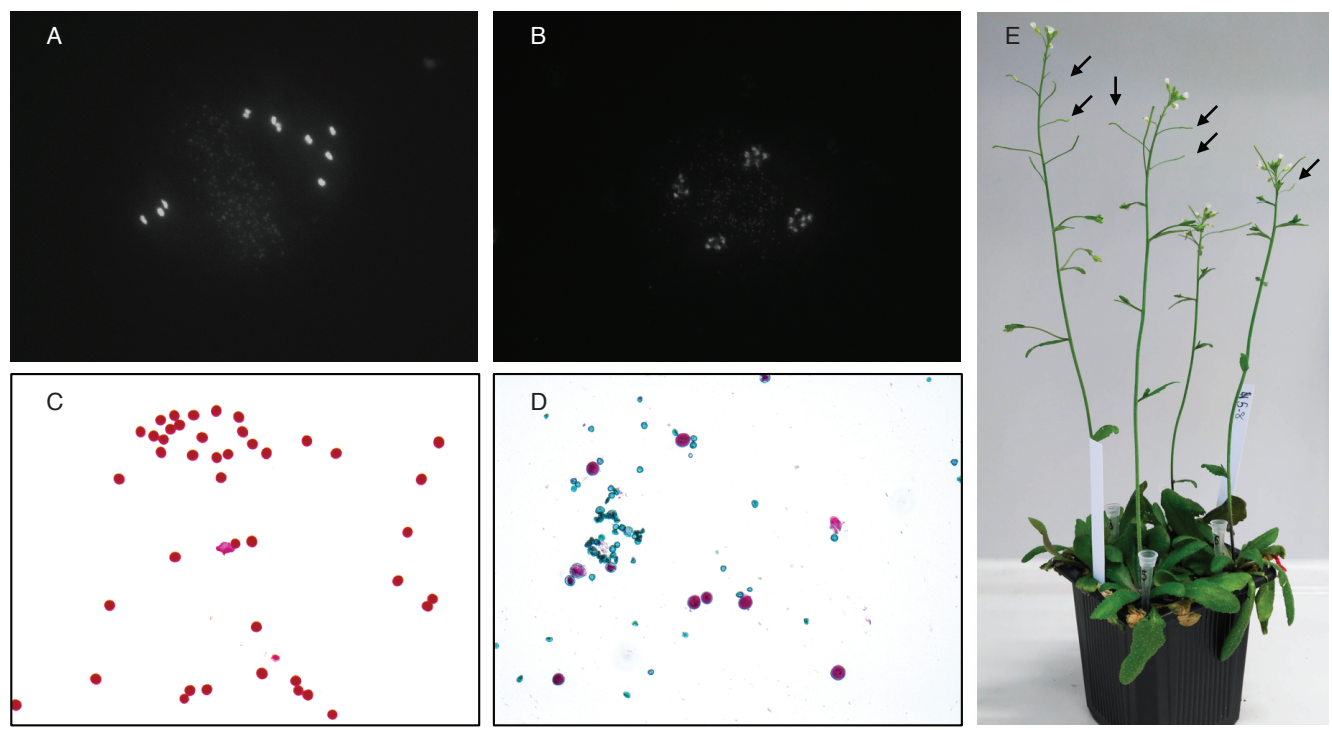

Supplementary figure 1. Inoculation with TRV2-AtMSH5 induces a phenocopy of a msh5 mutant phenotype in Arabidopsis thaliana hybrids during meiosis, in pollen phenotype and plant fertility. A and $B$ depict the result of random chromosome segregation at metaphase II (A) and tetrad stage (B) (same magnification). Pollen of a non-inoculated control plant is shown in (C). The result of a MSH5 knock-down leads to high pollen abortion in (D) (same magnification as C). MSH5 knocked-down plants display short siliques indicated with black arrows $(E)$ 


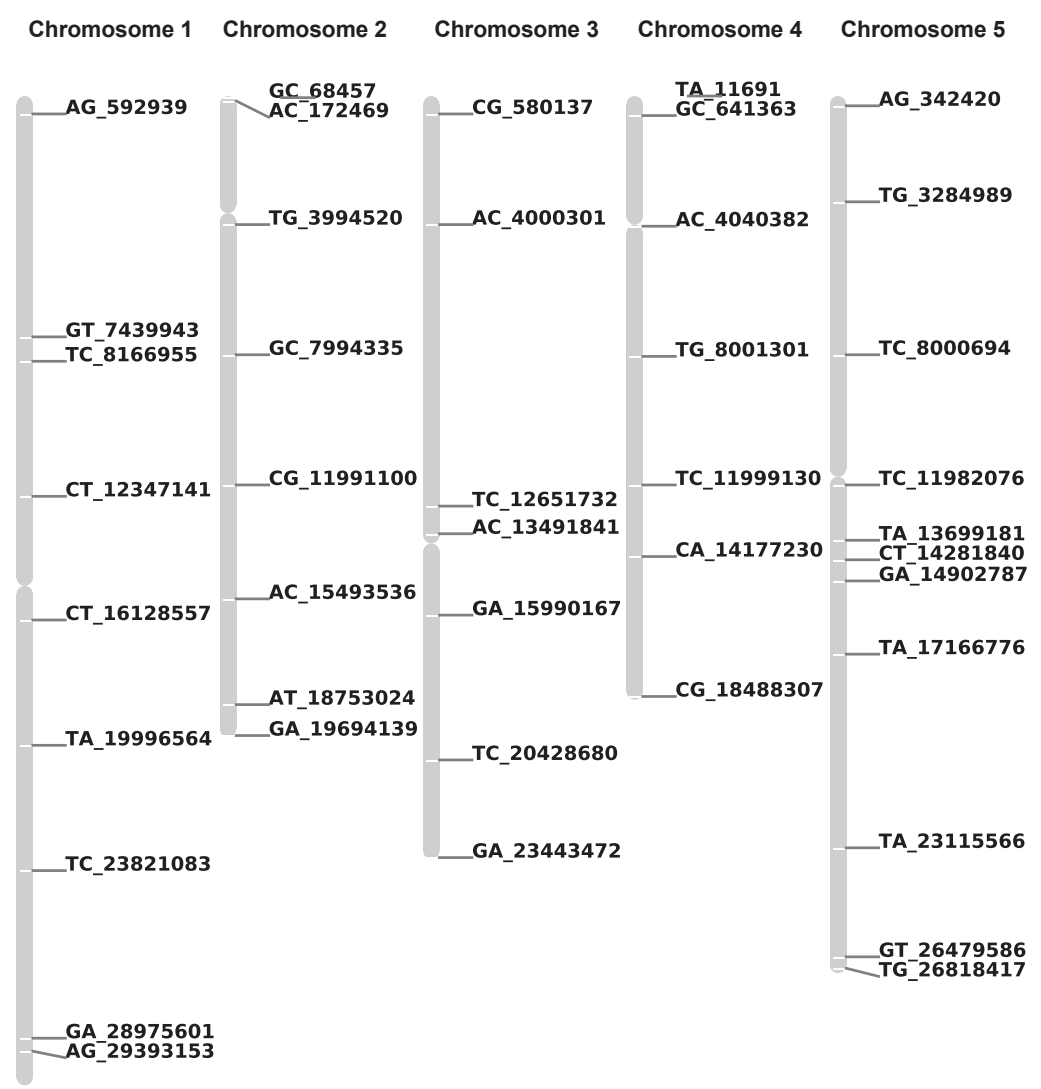

Supplementary figure 2. Physical positions of markers used to genotype reverse breeding offspring. The names of used markers indicate the Col-0 allele, the Ler allele and the bp position in the Col-0 reference genome. 

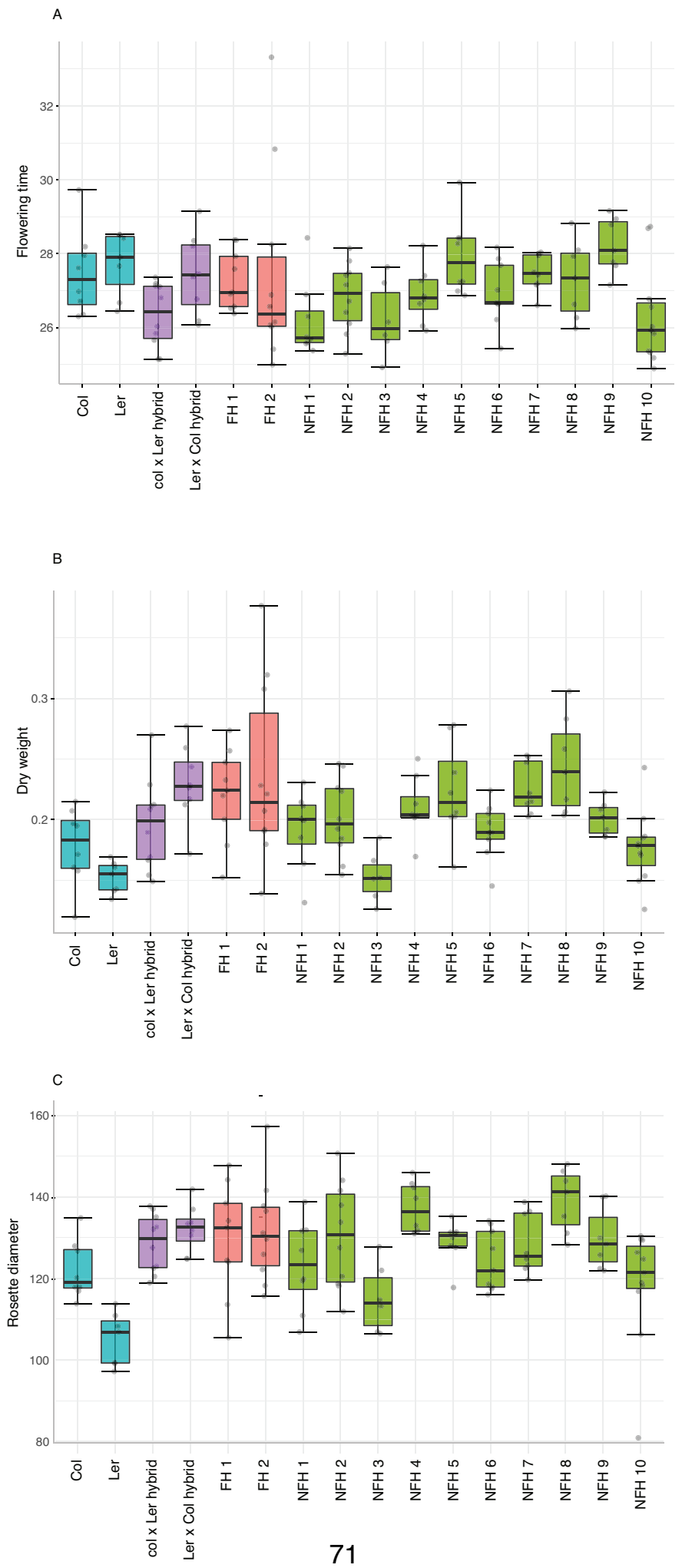
Supplementary figure 3. Box-plots illustrating the phenotypes of parental lines, full hybrids and partial hybrids for dry weight $(A)$, flowering time $(B)$ and rosette diameter $(C)$. From left to right boxplots are shown for the parental lines Col-0 and Ler (blue), Col-0 x Ler reciprocal hybrids (violet), full hybrids (FH, coral) and near-full hybrids ( $\mathrm{NFH}$, green). $\mathrm{FH}$ and $\mathrm{NFH}$ genotypes are shown in the supplementary file. The supplementary file can be retrieved from https://doi.org/10.1101/459016.

Supplementary tables 1-6. Expected number of $\mathrm{DH}_{0}$ and $\mathrm{DH}_{1}$ in $\mathrm{DH}$ populations with low crossover incidence. Tables indicate for different haploid chromosome numbers (1 ${ }^{\text {st }}$ row) the estimated numbers of crossovers per genome (CO/genome), the expected percentage of viable gametes and the expected percentage of $\mathrm{DH}_{0}$ and $\mathrm{DH}_{1}$ among $\mathrm{DH}$ offspring. The expected numbers of $\mathrm{DH}_{0}$ and $\mathrm{DH}_{1}$ are shown for the presence of $2 \mathrm{CO}, 1 \mathrm{CO}, 0.5 \mathrm{CO}, 0.2,0.1$ and $0 \mathrm{CO}$ ser bivalent pair in tables 1 through 6 respectively. In many plants, like in Arabidopsis, the typical number of COs per bivalent pair is between 2 and 1 (i.e. tables 1 and 2). In the case of complete $\mathrm{CO}$ suppression, no $\mathrm{DH}_{1}$ are recovered since no COs occur. For all calculations an exact (i.e. the indicated) number of COs per chromosome pair was assumed.

\begin{tabular}{|l|c|c|c|c|}
\hline \multicolumn{5}{|c|}{ Table 1: Cos per chromosome pair } \\
\hline $\mathbf{n}$ & $\mathbf{C O}$ / genome & Viable gametes (\%) & $\mathbf{D H}_{\mathbf{0}}(\%)$ & $\mathbf{D H}_{\mathbf{1}}(\%)$ \\
\hline $\mathbf{1}$ & 2 & 100 & 25,0 & 50,0 \\
\hline $\mathbf{2}$ & 4 & 100 & 6,25 & 25,0 \\
\hline $\mathbf{3}$ & 6 & 100 & 1,56 & 9,38 \\
\hline $\mathbf{4}$ & 8 & 100 & 0,39 & 3,13 \\
\hline $\mathbf{5}$ & 10 & 100 & 0,098 & 0,98 \\
\hline $\mathbf{6}$ & 12 & 100 & 0,024 & 0,29 \\
\hline $\mathbf{7}$ & 14 & 100 & 0,0061 & 0,085 \\
\hline $\mathbf{8}$ & 16 & 100 & 0,0015 & 0,024 \\
\hline $\mathbf{9}$ & 18 & 100 & 0,00038 & 0,0069 \\
\hline $\mathbf{1 0}$ & 20 & 100 & 0,000095 & 0,0019 \\
\hline $\mathbf{1 1}$ & 22 & 100 & 0,000024 & 0,00052 \\
\hline $\mathbf{1 2}$ & 24 & 100 & 0,0000060 & 0,00014 \\
\hline
\end{tabular}


Chapter 3 Efficient reverse breeding by VIGS-mediated transient crossover reduction

\begin{tabular}{|l|c|c|c|c|}
\hline \multicolumn{5}{|l|}{ Table 2: CO per chromosome pair } \\
\hline $\mathbf{n}$ & $\mathbf{C O}$ / genome & Viable gametes (\%) & $\mathbf{D H}_{\mathbf{0}}(\%)$ & $\mathbf{D H}_{\mathbf{1}}(\%)$ \\
\hline $\mathbf{1}$ & 1 & 100 & 50,0 & 50,0 \\
\hline $\mathbf{2}$ & 2 & 100 & 25,0 & 50,0 \\
\hline $\mathbf{3}$ & 3 & 100 & 12,5 & 37,5 \\
\hline $\mathbf{4}$ & 4 & 100 & 6,25 & 25,0 \\
\hline $\mathbf{5}$ & 5 & 100 & 3,13 & 15,6 \\
\hline $\mathbf{6}$ & 6 & 100 & 1,56 & 9,38 \\
\hline $\mathbf{7}$ & 7 & 100 & 0,78 & 5,47 \\
\hline $\mathbf{8}$ & 8 & 100 & 0,39 & 3,13 \\
\hline $\mathbf{9}$ & 9 & 100 & 0,20 & 1,76 \\
\hline $\mathbf{1 0}$ & 10 & 100 & 0,10 & 0,98 \\
\hline $\mathbf{1 1}$ & 11 & 100 & 0,049 & 0,54 \\
\hline $\mathbf{1 2}$ & 12 & 100 & 0,024 & 0,29 \\
\hline
\end{tabular}

\begin{tabular}{|l|c|c|c|c|}
\hline \multicolumn{5}{|c|}{ Table 3: Cos per chromosome pair } \\
\hline $\mathbf{n}$ & $\mathbf{C O}$ / genome & Viable gametes (\%) & $\mathbf{D H}_{\mathbf{0}}$ (\%) & $\mathbf{D H}_{\mathbf{1}}$ (\%) \\
\hline $\mathbf{1}$ & 0,5 & 75,0 & 75,0 & 25,0 \\
\hline $\mathbf{2}$ & 1 & 50,0 & 56,3 & 37,5 \\
\hline $\mathbf{3}$ & 1,5 & 37,5 & 42,2 & 42,2 \\
\hline $\mathbf{4}$ & 2 & 25,0 & 31,6 & 42,2 \\
\hline $\mathbf{5}$ & 2,5 & 18,8 & 23,7 & 39,6 \\
\hline $\mathbf{6}$ & 3 & 12,5 & 17,8 & 35,6 \\
\hline $\mathbf{7}$ & 3,5 & 9,38 & 13,3 & 31,1 \\
\hline $\mathbf{8}$ & 4 & 6,25 & 10,0 & 26,7 \\
\hline $\mathbf{9}$ & 4,5 & 4,69 & 7,51 & 22,5 \\
\hline $\mathbf{1 0}$ & 5 & 3,13 & 5,63 & 18,8 \\
\hline $\mathbf{1 1}$ & 5,5 & 2,34 & 4,22 & 15,5 \\
\hline $\mathbf{1 2}$ & 6 & 1,56 & 3,17 & 12,7 \\
\hline
\end{tabular}




\begin{tabular}{|l|c|c|c|c|}
\hline \multicolumn{5}{|l|}{ Table 4: $\mathbf{0}$ per chromosome pair } \\
\hline $\mathbf{n}$ & $\mathbf{C O}$ / genome & Viable gametes (\%) & $\mathbf{D H}_{\mathbf{0}}(\%)$ & $\mathbf{D H}_{\mathbf{1}}(\%)$ \\
\hline $\mathbf{1}$ & 0,2 & 60,0 & 90,0 & 10,0 \\
\hline $\mathbf{2}$ & 0,4 & 35,0 & 81,0 & 18,0 \\
\hline $\mathbf{3}$ & 0,6 & 20,0 & 72,9 & 24,3 \\
\hline $\mathbf{4}$ & 0,8 & 11,3 & 65,6 & 29,1 \\
\hline $\mathbf{5}$ & 1 & 6,25 & 59,0 & 32,8 \\
\hline $\mathbf{6}$ & 1,2 & 3,75 & 53,1 & 35,4 \\
\hline $\mathbf{7}$ & 1,4 & 2,19 & 47,8 & 37,2 \\
\hline $\mathbf{8}$ & 1,6 & 1,25 & 43,0 & 38,2 \\
\hline $\mathbf{9}$ & 1,8 & 0,70 & 38,7 & 38,7 \\
\hline $\mathbf{1 0}$ & 2 & 0,39 & 34,9 & 38,7 \\
\hline $\mathbf{1 1}$ & 2,2 & 0,23 & 31,4 & 38,3 \\
\hline $\mathbf{1 2}$ & 2,4 & 0,14 & 28,2 & 37,6 \\
\hline
\end{tabular}

\begin{tabular}{|l|c|c|c|c|}
\hline \multicolumn{5}{|c|}{ Table 5: CO per chromosome pair } \\
\hline $\mathbf{n}$ & $\mathbf{C O}$ / genome & Viable gametes (\%) & $\mathbf{D H}_{\mathbf{0}}(\%)$ & $\mathbf{D H}_{\mathbf{1}}(\%)$ \\
\hline $\mathbf{1}$ & 0,1 & 55,0 & 95,0 & 5,00 \\
\hline $\mathbf{2}$ & 0,2 & 30,0 & 90,3 & 9,50 \\
\hline $\mathbf{3}$ & 0,3 & 16,3 & 85,7 & 13,5 \\
\hline $\mathbf{4}$ & 0,4 & 8,75 & 81,5 & 17,1 \\
\hline $\mathbf{5}$ & 0,5 & 4,69 & 77,4 & 20,4 \\
\hline $\mathbf{6}$ & 0,6 & 2,50 & 73,5 & 23,2 \\
\hline $\mathbf{7}$ & 0,7 & 1,33 & 69,8 & 25,7 \\
\hline $\mathbf{8}$ & 0,8 & 0,70 & 66,3 & 27,9 \\
\hline $\mathbf{9}$ & 0,9 & 0,37 & 63,0 & 29,9 \\
\hline $\mathbf{1 0}$ & $\mathbf{1}$ & 0,20 & 59,9 & 31,5 \\
\hline $\mathbf{1 1}$ & 1,1 & 0,11 & 56,9 & 32,9 \\
\hline $\mathbf{1 2}$ & 1,2 & 0,06 & 54,0 & 34,1 \\
\hline
\end{tabular}




\begin{tabular}{|l|c|c|c|c|}
\hline \multicolumn{5}{|c|}{ Table 6: CO per chromosome pair } \\
\hline $\mathbf{n}$ & $\mathbf{C O}$ / genome & Viable gametes (\%) & $\mathrm{DH}_{\mathbf{0}}(\%)$ & $\mathrm{DH}_{\mathbf{1}}(\%)$ \\
\hline $\mathbf{1}$ & 0 & 50,0 & 100,0 & 0,00 \\
\hline $\mathbf{2}$ & 0 & 25,0 & 100,0 & 0,00 \\
\hline $\mathbf{3}$ & 0 & 12,5 & 100,0 & 0,00 \\
\hline $\mathbf{4}$ & 0 & 6,25 & 100,0 & 0,00 \\
\hline $\mathbf{5}$ & 0 & 3,13 & 100,0 & 0,00 \\
\hline $\mathbf{6}$ & 0 & 1,56 & 100,0 & 0,00 \\
\hline $\mathbf{7}$ & 0 & 0,78 & 100,0 & 0,00 \\
\hline $\mathbf{8}$ & 0 & 0,39 & 100,0 & 0,00 \\
\hline $\mathbf{9}$ & 0 & 0,20 & 100,0 & 0,00 \\
\hline $\mathbf{1 0}$ & 0 & 0,10 & 100,0 & 0,00 \\
\hline $\mathbf{1 1}$ & 0 & 0,049 & 100,0 & 0,00 \\
\hline $\mathbf{1 2}$ & 0 & 0,024 & 100,0 & 0,00 \\
\hline
\end{tabular}


To confidence and creativity.

"It always seems impossible until it's done".

Nelson Mandela. 


\section{Chapter 4}

\section{VIGS-mediated increase of recombination in Arabidopsis thaliana.}

Authors.

Vanesa Calvo-Baltanás ${ }^{1}$, Max Van der Heide², C. Bastiaan de Snoo³, Arp Schnittger², Hans de Jong ${ }^{1}$, Erik Wijnker ${ }^{1}$.

\section{Authors affiliations.}

1) Laboratory of Genetics, Wageningen University \& Research, P.O. Box 166700 AA Wageningen, The Netherlands.

2) Department of Developmental Biology, University of Hamburg, Institute for plant sciences and microbiology, 22609 Hamburg, Germany.

3) Rijk Zwaan R\&D Fijnaart, Eerste Kruisweg 9, 4793 RS Fijnaart, The Netherlands. 


\section{Abstract.}

The ability to engineering meiotic recombination is considered essential for plant breeding to, for instance, speed up the production of desirable allele combinations of tightly linked genes or to increase the resolution of mapping populations. Recent studies in plant meiosis have revealed that RECQ4 and FIGL1 limit the number of crossovers through two independent anti-crossover pathways. However, these genes are also important for DNA repair and, likely as a consequence of that, for pollen viability. Here, we explore the use of virus-induced gene silencing (VIGS) to increase crossover frequencies in the model organism Arabidopsis thaliana by targeting RECQ4 and FIGL1. We show that silencing of these factors presumably leads to increased meiotic crossover recombination, inferred from the phenocopy of the figl1 recq4 mutant phenotype observed in meiotic chromosome spreads. We used F1 hybrids inoculated with a VIGS constructs to generate a backcross population, but the lack of high recombinant offspring suggests that the VIGS knockdown of RECQ4 and/or FIGL1 causes additional defects, precluding the formation of hyper-recombinant offspring. These results are in line with studies in other plants species that have reported male sterility or reduced fertility of recq4 and figl1 mutants. Null-mutants and/or complete knockdowns of these genes should be generated and thoroughly investigated to understand the function of RECQ4 and FIGL1, not only in meiosis but also in somatic cells.

\section{Introduction.}

Plant breeding relies on repeated cycles of interbreeding of individuals followed by choosing the combination of favorable traits in the offspring. In every generation new allele combinations are generated through meiotic recombination. Some of these allele combinations may be selected if they are favorable for breeding purposes. Also, thanks to recombination, mapping populations can be created and used to identify the loci that encode favorable traits, which can be then introgressed into existing breeding lines. In this introgression process, breeders benefit from selecting crossover events close to the locus of interest, to reduce the concomitant introgression of other (potentially nonfavorable) alleles. This process can be facilitated if higher recombination rates exist in the population. The likelihood of recovering crossover events close to the focal locus is limited by the crossover rate in the chromosome region of interest. Increasing crossovers in such a chromosome segment enables faster recovering of favorable recombination events, and so would dramatically reduce the number of plants screened for this purpose. An increase in meiotic crossover formation would also be favorable in the generation of mapping populations themselves. The more recombination events occur and the more loci independently segregate, the higher is the mapping resolution (Korte and Farlow, 2013).

Meiotic recombination is a tightly regulated process in which usually one or two crossovers (COs) per chromosome in plants are formed (Wijnker and de Jong, 2008). 
In plants, two different crossovers (hereafter COs) pathways co-exist (Mercier et al., 2005; Falque et al., 2009; Lambing, Franklin and Wang, 2017). The class I crossover pathway accounts for about $80 \%-85 \%$ of the total number of COs. Class I COs are processed by the ZMM (ZIP, MSH, MER) pathway and are interference dependent, meaning that they are more distantly situated from one another than expected by random placement (Copenhaver, Housworth and Stahl, 2002; Mercier et al., 2005; Lynn, Soucek and Börner, 2007; Higgins et al., 2008; Lu et al., 2008; Falque et al., 2009). The dramatic decrease of COs in $\mathrm{zmm}$ mutants leads to the formation of aneuploid gametes, causing semi-sterility in the mutant background. Class II COs involve the endonuclease MUS81 and account for the remaining 20\%-15\% of all COs (Mercier et al., 2005; Berchowitz et al., 2007). Contrary to type I COs, type II COs are interferenceindependent and are randomly placed along the chromosome axis (Berchowitz et al., 2007; Lambing, Franklin and Wang, 2017). The frequency of class II COs is negatively regulated by three independent pathways: the protein complex formed by AAA-ATPase FIDGETIN-LIKE 1 and FIDGETINE-LIKE 1 INTERACTING PROTEIN (FIGL1-FLIP), FANCONI ANEMIA COMPLEMENTATION GROUP M-LIKE PROTEIN (FANCM) and RECQ Helicase IV (RECQ4). These genes were identified as CO repressors through a suppressor screen of zmm mutants. Mutations in FIGL1, RECQ4 or FANCM were found to restore COs frequencies to almost wild-type levels and rescue the semi-sterility of zmm mutants (Crismani et al., 2012; Girard et al., 2015; Séguéla-Arnaud et al., 2015; Fernandes et al., 2017).

FIGL1 was first characterized in Arabidopsis and together with FLIP, they form a complex that regulates strand invasion during homologous recombination $(\mathrm{HR})$, in both somatic and meiotic cells (Fernandes et al., 2018). Although the exact mechanism is to be elucidated, it was proposed that FIGL1 regulates HR by controlling RADIATION SENSITIVE 51 (RAD51) and DISRUPTED MEIOTIC cDNA 1 (DMC1) dynamics and/or numbers (Girard et al., 2015; Fernandes et al., 2018). In Arabidopsis Y2H experiments revealed the interaction between FIGL1 and RAD51 via the orthologue to the FIDGETIN 1 - RAD51 Binding Domain (FRBD) of FIGL1 and the interaction between FIGL1 and DMC1 (Fernandes et al., 2018). Interestingly, none of the described figl1 mutant alleles in Arabidopsis is mutated in the FRBD domain (Figure 1) (Girard et al., 2015).

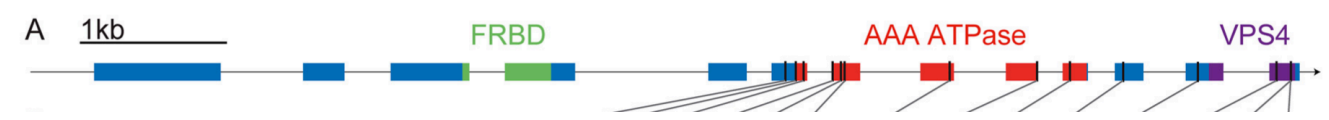

Figure 1 Point mutation positions in AtFIGL, each of them represented by a black line. Note that none of the point mutations are found in or upstream the FRBD domain required for the interaction between FIGL1 and RAD51 and likely for the interaction between FIGL1-DMC1. (Modified from Giraud et al., 2015). 
The RECQ4 function in Arabidopsis is represented by two genes: RECQ4A and $R E C Q 4 B$. Although they share high sequence similarity, they appear to have some functional differences (Schröpfer et al., 2014; Séguéla-Arnaud et al., 2017). The RECQ4A protein limits crossover formation by controlling homologous recombination (HR) during meiosis, it is also involved in HR in somatic cells (Mannuss et al., 2010) and has further functions in DNA repair and replication (Hartung, Suer and Puchta, 2007; Knoll and Puchta, 2011; Schröpfer et al., 2014; Séguéla-Arnaud et al., 2017). It contains two domains essential for these different processes: the helicase domain and the $\mathrm{N}$ terminal region (Schröpfer et al., 2014). Schröpfer et al., 2014, showed that the Nterminal region together with its helicase domain are required in Arabidopsis not only for HR but also for DNA repair, and indicated that RECQ4A may also suppress HR in both a helicase and an $\mathrm{N}$-terminus-dependent manner (Schröpfer et al., 2014). On the other hand, although RECQ4B, similar to RECQ4A, also limits class II meiotic crossovers (Séguéla-Arnaud et al., 2015, 2017), it is apparently not essential for DNA repair (Hartung, Suer and Puchta, 2007).

The double mutants fancm recq4 and figl1 recq4 lead to about 10-fold increase of recombination frequencies in a Col-0 background compared to wild-type Col-0. In the Col-0/Ler background, the highest recorded increase in recombination was reported for the double mutant figl1 recq4 with a 7.8-fold increase genome wide as compared to the wild-type (Fernandes et al., 2017). This double mutant displays high rates of pollen abortion in both the Col- 0 accession ( $>40 \%$ pollen abortion) and the Ler-Col hybrid ( $<40 \%$ pollen abortion). Yet, despite the decrease in pollen viability, seed set was not significantly reduced in figl1 recq4 in a Ler-Col hybrid background. In contrast, seed set was significantly reduced in figl1 recq4 Col-0 (Fernandes et al., 2017). This decrease in fertility may be partially explained by the abnormalities observed in meiosis in figl1 recq4 mutants. Chromosomes in diakinesis seem to be more "tightly linked" than in wild-type diakinesis, but align normally on the metaphase plate and show balanced segregation during anaphase I (Fernandes et al., 2017). Metaphase bivalents have an atypical form during metaphase I in which "rod" or "ring" bivalents are formed (homologs connected by one or two COs respectively). Instead, the tight connections between homologs are also maintained in metaphase I. However, in figl1 recq4 mutants, chromosome fragments and chromosome bridges are visible in about $50 \%$ of metaphase II cells (Fernandes et al., 2017).

The fig1 mutants in rice and pea are both known as male sterile and consequently an increase of recombination frequencies was not assessed in these mutants (Zhang et al., 2017; Mieulet et al., 2018). On the other hand, recq4 mutants in pea, rice, and tomato displays an increase of recombination frequencies (Mieulet et al., 2018) and suggests a conserved function of this gene in limiting crossover formation. Therefore, for plant breeding, a controlled increase of recombination frequencies by mutating or downregulating the expression of RECQ4 could be applied as an universal tool in the actual 
generation of mapping populations or in the generation of novel allelic combinations (Mieulet et al., 2018). Techniques to rapidly modify gene expression and generate transgene-free offspring would be the most efficient and the preferred approach to generate and obtain highly recombinant offspring. Crucially, it has now been shown that virus-induced gene silencing (VIGS) is a transient gene silencing approach that can be used to efficiently downregulate the expression of meiotic genes in Arabidopsis hybrids (Chapter 2 and 3, this thesis). Importantly, VIGS systems are available for various crops (Senthil-Kumar and Mysore, 2011; Becker, 2013) so that the use of VIGS could be applied to directly increase recombination frequencies and to gain insight in the function of genes that limit crossover formation in crops, in addition to Arabidopsis.

We hypothesized that an increase of recombination frequencies can be achieved by targeting FIGL1 and RECQ4 using a TRV2-based VIGS system. We also tested whether a simultaneous knock-down of these two genes during meiosis was possible with TRV2 to increase COs frequencies. Because VIGS induced phenotypes are not fully penetrant, silenced and non-silenced gametes usually appear in the same flower (Chapter 2, this thesis). We therefore decided to include the sequence of a third target gene in the VIGSconstruct that could serve as phenotypic marker for pollen to select only silenced pollen grains. In Chapter 2, we already showed that a single knockdown of QRT1 and QRT2 using VIGS led to the production of pollen tetrads, but this was never tested in combination with other target genes. To this end, we created a triple construct TRV2RECQ4-FIGL1-QRT2 (hereafter TRV2-RFQ) to simultaneously target the three mentioned genes. Furthermore, we also tested whether high-recombinant offspring could be obtained from plants inoculated with TRV2-RFQ. The results obtained in our experiments suggest that RECQ4 and FIGL1 in Arabidopsis may be pleiotropic genes, essential not only to limit crossover formation but also to ensure gamete viability.

\section{Results.}

1. VIGS against $F I G L 1$ and RECQ4 can partially rescue a $z m m$ mutant phenotype.

To assess the possibility of transiently increasing crossover recombination using a TRV2 VIGS vector, we first tested the potential of VIGS to induce an increase of crossovers by silencing RECQ4 (RECQ4A and RECQ4B) and FIGL1. We hypothesized that we could verify this by inoculating mutants of the ZMM gene MUT S HOMOLOG4 (MSH4). Silencing of RECQ4 or FIGL1 in a msh4 mutant background should then rescue the semi-sterile phenotype displayed by msh4 (Girard et al., 2015; Séguéla-Arnaud et al., 2015). To this end, we first designed our VIGS constructs using the online tool SGN VIGS (Fernandez-Pozo et al., 2015). This allowed us to identify the best possible FIGL1 and RECQ4 target sequences and to exclude possible off-targets (see materials and methods). We first selected two regions of RECQ4A as reference sequences and then used TAIR BLAST to validate that the selected sequences shared homology with 
RECQ4B. The two sequences selected to target RECQ4A shared $86 \%$ and $90 \%$ sequence similarity with $R E C Q 4 B$ respectively, and hence were likely to downregulate both genes simultaneously. Two different cDNA sequences of 194 and $166 \mathrm{bp}$ that are homologous to RECQ4 were then inserted into TRV2, obtaining TRV2-RECQ4_1 and TRV-RECQ4_2. To create TRV2-FIGL1, we used a FIGL1 cDNA sequence of $169 \mathrm{bp}$. All the TRV2 constructs were then cloned into the Agrobacterium tumefaciens strain GV3101 and used for subsequent agroinoculation.

We agroinoculated homozygous msh4 mutants (Col-0 background) at the 4-5 leaf stage with TRV2-FIGL1, TRV2-RECQ4_1 and TRV2-RECQ4_2, and monitored the development of the plants. A msh4 mutant displays mainly short-siliques as a consequence of low gamete viability of both the female and the male gametophytes. We observed that in inoculated plants, some siliques elongated in contrast to the short siliques formed in msh4 (see Figure 2). This indicates that we could partially restore fertility of msh4 by silencing FIGL1 or RECQ4, and, hence, had likely increased crossover numbers during female and possibly also during male meiosis in msh4 mutants.

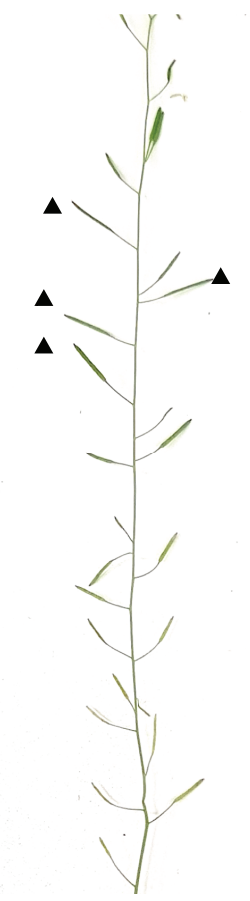

msh4 TRV2-FIGL1

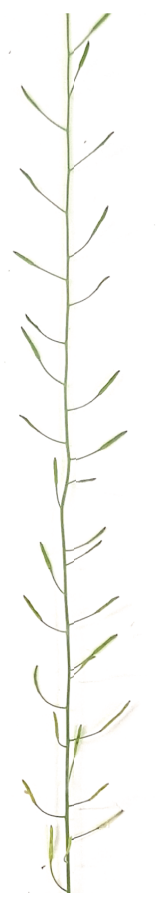

Control msh4

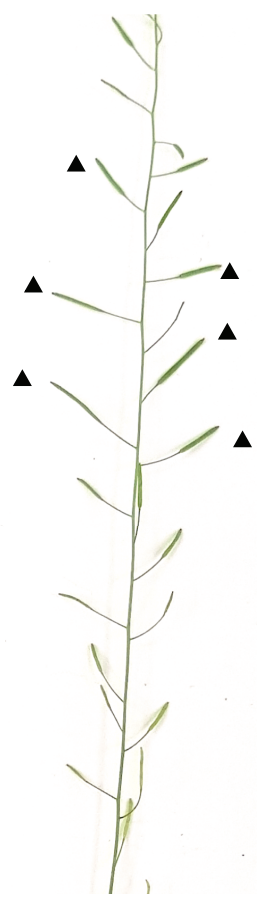

msh4 TRV2-RECQ4_2

Figure 2: Col-0 msh4 mutants show a rescue of their semi-sterile phenotype when FIGL1 and RECQ4 are silenced by VIGS. From left to right a comparison of the phenotypes is shown between a TRV2FIGL1 knockdown plant, a control msh4 and a msh4 TRV2-RECQ4 knockdown. Black triangles indicate which siliques elongated after treatment. 
2. Generation of a VIGS construct to target FIGL1-RECQ4 coupled to a pollen phenotypic marker: QRT2.

Since the double mutant figl1 recq4 shows the highest increase in recombination frequencies in a hybrid background compared to all the single mutants so far described (Fernandes et al., 2017), we decided to use one VIGS vector to simultaneously target RECQ4 and FIGL1 in an F1 Columbia x Landsberg erecta hybrid and, as positive control, in Col-0 plants too. A successful silencing of RECQ4-FIGL1 can be initially validated if pollen abortion occurs in inoculated plants, as described for figl1 recq4 (Fernandes et al., 2017). Furthermore, we also decided to concomitantly knock-down a post-meiotic gene, QUARTET 2 (QRT2) together with FIGL1 and RECQ4, to induce pollen tetrads that could serve as a macroscopic marker to select for viable VIGSderived pollen grains. By targeting QRT2 together with FIGL1 and RECQ4, we expected to see pollen tetrads and pollen abortion in flowers affected by the silencing. We then generated a triple VIGS construct harboring three cloned CDNA sequences homologous to RECQ4, FIGL1 and QRT2, obtaining an insert of $622 \mathrm{bp}$ in length and then generating TRV2-RECQ4-FIGL1-QRT2 (hereafter TRV2-RFQ).

3. Simultaneous knockdown of FIGL1 and RECQ4 in Col-0 can phenocopy the mutant phenotype observed in meiotic chromosome spreads of figl1 recq4.

We used the VIGS vector TRV2-RFQ to inoculate Col-0 plants at 4-5 leaf stage and evaluated if they resembled the figl1 recq4 phenotype. To assess meiotic progression in treated plants, we performed chromosome spread analyses on $27 \mathrm{Col}-0$ inflorescences sampled at 18 days post-inoculation (d.p.i). Flower buds of Col-0 wildtype plants were also sampled and used for comparison. In each inflorescence harvested, only one flower bud was expected to have anthers with pollen mother cells at meiosis, so the total sample size comprised 27 flower buds. Out of these 27 flower buds, we found one in which silenced cells by TRV2-RFQ resembled the meiotic mutant phenotype described by Fernandes et al., 2017. We evaluated the meiocytes in the silenced flower bud that contained a total of 197 pollen mother cells and a comparable number of 114 cells for the wild-type control. The first meiotic stage at which we observed differences between silenced and wild-type cells was late diakinesis. Wild-type cells in diakinesis display ring or rod-shaped bivalents in which the homologs are connected to each other mostly with clearly separated chiasmata producing open structures between the arms in the chromosome regions that have no COs. These rod or ring bivalents result from one or two chiasmatic connections, respectively. In TRV2RFQ cells in diakinesis, $100 \%$ of cells $(n=47)$ showed bivalents in which homologs looked tightly linked to each other (Figure 3). In meiotic chromosome spreads of $100 \%$ of the silenced cells $(n=5)$ in metaphase I, tightly condensed bivalents appear, likely corresponding to an increased number of chiasmata, as described by Fernandes et al., 2017. In wild-type cells in metaphase II, no chromosome fragments were found, while in 
$28 \%$ of the silenced cells $(n=5)$ in metaphase II we observed chromosome fragments. The occurrence of these phenotypes (Fernandes et al., 2017) show that high levels of recombination exist in inoculated plants with TRV2-RFQ. We did not observe further defects in other meiotic stages and cells seem to proceed normally to tetrad-stage.
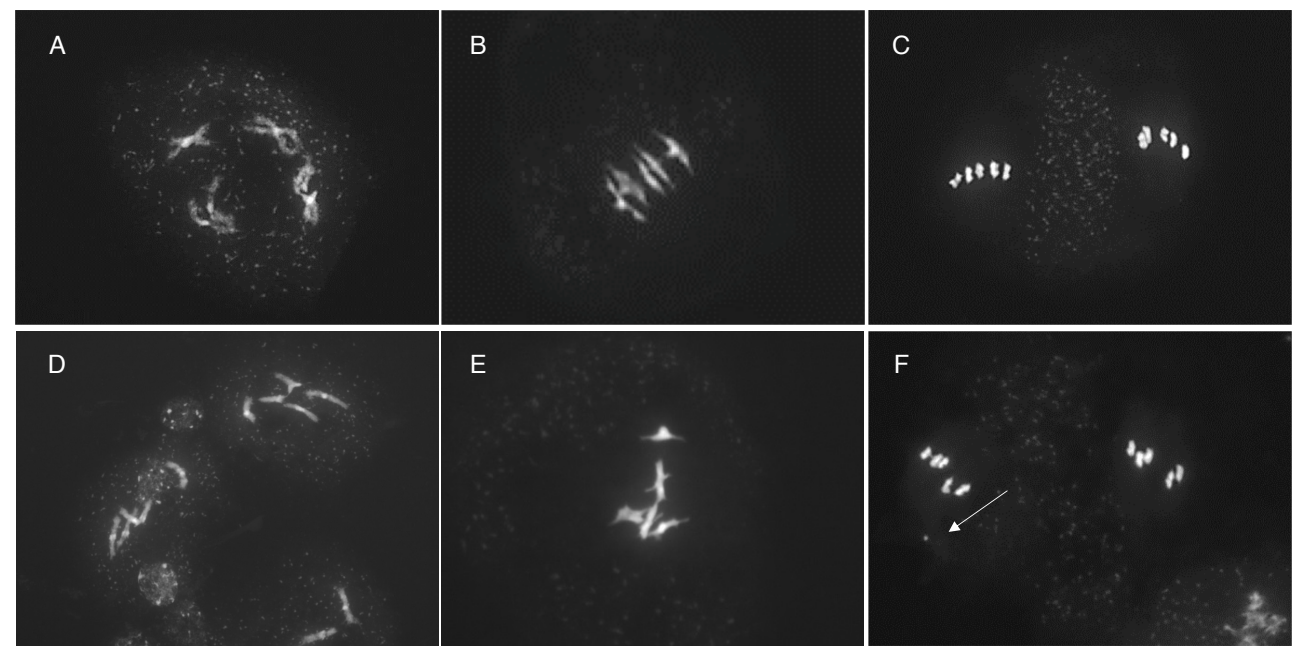

Figure 3. Meiotic chromosome spreads of Col-0 wild-type plants and plants inoculated with the TRV2RFQ VIGS construct. A-C shows wild-type meiocytes at diakinesis (A), metaphase I (B) and metaphase II (C). Lower row depicts meiotic cells spreads in plants inoculated with TRV2-RFQ. Chromosomes in late diakinesis (D) appear as tightly connected structures, which can still be recognized still during metaphase I (E). In Metaphase II (F), although balanced chromosome segregation occurred, a small chromosome fragment is visible (white arrow).

4. FIGL1-RECQ4 knockdown plants display semi-sterility.

We also evaluated the pollen phenotype of $\mathrm{F} 1$ and Col-0 plants inoculated with TRV2RFQ, to see whether, 1) the VIGS inoculated plants showed a pollen abortion phenotype as reported for the double figl1 recq4 mutant (Fernandes et al., 2017) and/or 2) if pollen tetrads were formed. Furthermore, it is interesting to determine a possible correlation between aborted and pollen tetrads, and also if we could observe differences in the penetrance between targets (i.e. only pollen tetrads and no aborted pollen or vice versa). Staining pollen from F1 and Col-0 flowers allowed us to discern between aborted and viable pollen (Figure 4). About three weeks post-inoculation (w.p.i) we observed a pollen abortion phenotype in TRV2-RFQ inoculated plants (Figure 4). However, we did not observe any pollen tetrad - viable or aborted - pollen. The absence of pollen tetrads meant that we could not visually select for pollen affected by silencing of FIGL1 and RECQ4 either. Because previous experiments showed that VIGS penetrance is rarely complete, we were not able to determine which ones may carry high-recombinant chromosomes and which ones are wild-type pollen grains. 
To examine the consequences of pollen abortion on seed production, we monitored silique development in Col-0 treated plants. In contrast to the controls, Col-0 inoculated plants with TRV2-RFQ exhibited short siliques along the main stem that may possibly have been caused by low pollen viability (Fernandes et al., 2017) (See Figure 4). In the figl1 recq4 double mutant, a significant reduction in the seed-set (Anova; $p<0,001$ ) was also observed in Col-0, but not in a Ler-Col hybrid background (Fernandes et al., 2017). Here, the formation of short siliques on knockdown plants suggests that inoculation with TRV2-RFQ has an effect on pollen viability and plant fertility.
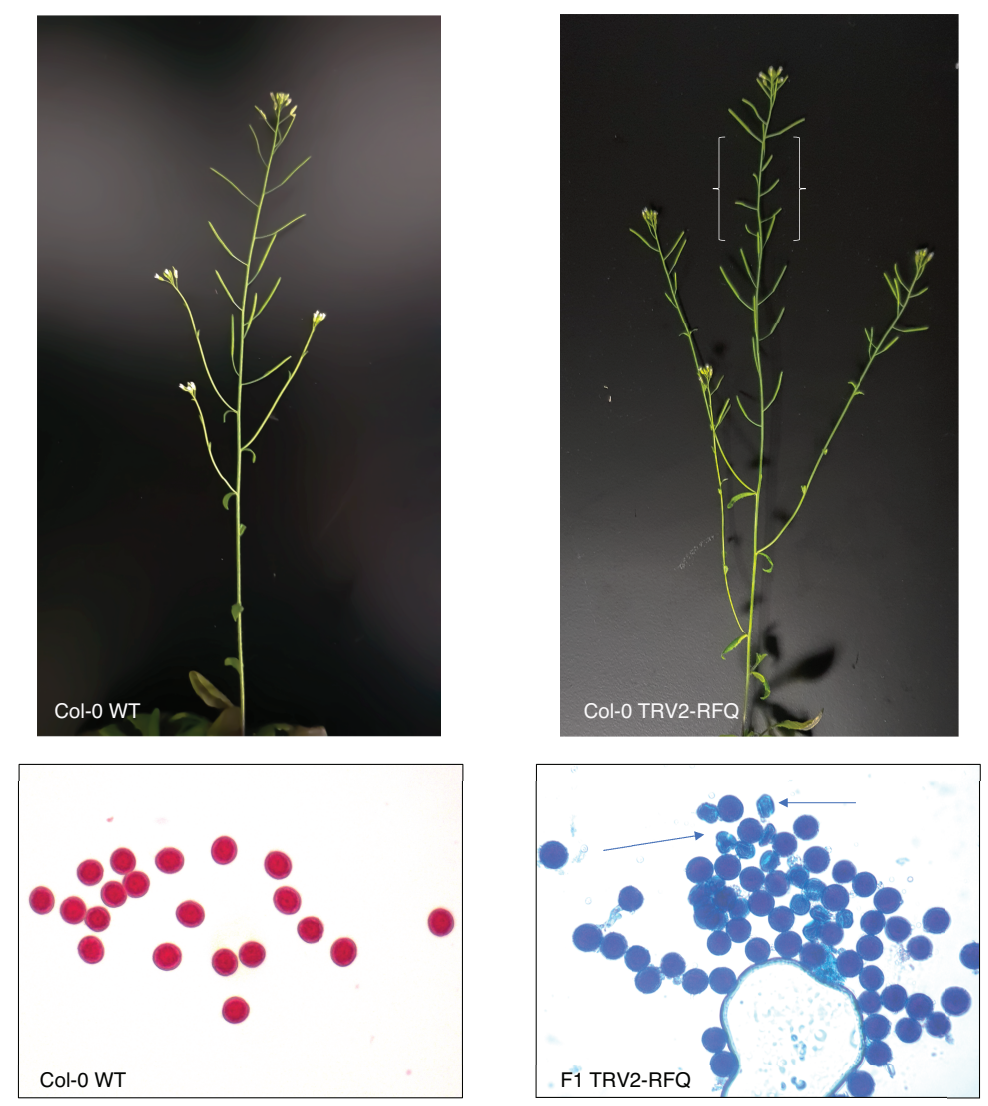

Figure 4: Semi-sterility displayed by TRV2-RFQ inoculated plants. Upper row; silique development in Col-0 control (left) compared to Col-0 inoculated with TRV2-RFQ (right). While the siliques in the Col-0 control elongate normally, the inoculated plant displays several short siliques in a row along the main stem corresponding to a semi-sterile phenotype likely due to poor pollen viability, that reverts to a wild-type phenotype later, overcoming the silencing effect (see the plant section in between white accolades). Lower row; stained pollen of Arabidopsis plants under the same magnification. On the left, viable pollen in Col-0 wild-type, and on the right, aborted pollen (arrows) in an F1 hybrid inoculated with TRV2-FRQ. 
5. High-recombinant offspring could not be recovered but lower interference is observed in the VIGS derived population compared to the wild-type control population.

To determine whether plants inoculated with TRV2-RFQ could produce high recombinant offspring, we selected 35 flowers exhibiting strong pollen abortion (taken from 22 inoculated $\mathrm{F} 1$ hybrid plants) to pollinate male sterile ( $m s)$ Ler mutant plants for producing a $\mathrm{BC} 1$ population. The recovered progeny was genotyped for a total of 48 SNP markers using KASPAR probes spaced over the five chromosomes of Arabidopsis thaliana (see Figure 5). A total of $82 \mathrm{BC} 1$ plants were genotyped together with 109 control wild-type offspring, and the distributions of observed COs per line in the BC1 are shown in Figure 5. We tested whether there were significant differences between both distributions using a Kolmogorov-Smirnov test, but none were found ( $p$-value $>0,05$ ).

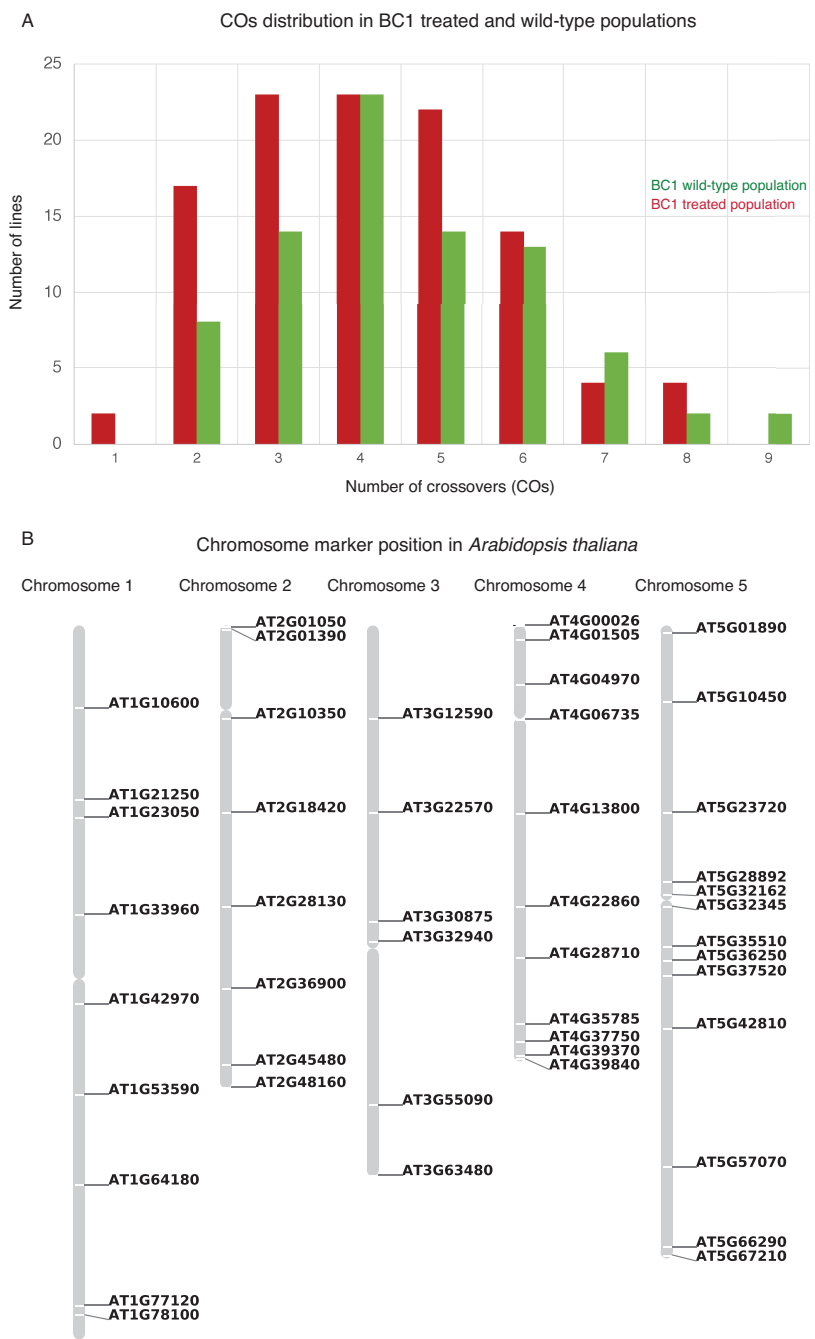


Figure 5: A) Graph showing the number crossovers in BC1 WT lines (green) and BC1 offspring derived from VIGS treated plants (red). Using ( $n$ ) of $n B C 1$ treated $=109$ and $n B C 1$ wild-type $=83$, no increase in crossover number was found for this data set. The differences between both distributions was not significant (Statistical test Kolmogorov-Smirnov alpha $=0.05 ; p=0.2$ ). B) Graphical representation of the 48 markers position used for the genotyping of the $\mathrm{BC} 1$ offspring in the five chromosomes of Arabidopsis thaliana. Two markers are placed in AT1G10600 but it is represented in the figure as a single marker.

\section{Discussion.}

We hypothesized that recombination frequencies could be transiently increased by downregulating expression of FIGL1 and RECQ4 using VIGS. Furthermore, we also aimed to test if simultaneous silencing of FIGL1, RECQ4, and QRT2 as a phenotypic marker to identify pollen resulting from silenced cells, could be achieved using a single combined VIGS construct. We observed a phenocopy of the described figl1 recq4 meiotic phenotype and we also observed pollen abortion in TRV2-RFQ inoculated plants. However, we did not observe pollen tetrads nor recovered high recombinant offspring. Because the formation of pollen tetrads was previously validated in a TRV2QRT2 knockdown (Chapter 2, this thesis) and offspring and high-recombinant gametes could be obtained from figl1 recq4 Arabidopsis mutants (Chapters 2 and 3), we will first discuss possible reasons why we did not observe these two phenotypes in knockeddown plants. Next, we will compare the phenotypes obtained in TRV2-RFQ inoculated plants with figl1 and recq4 mutants in other plant species. Finally, we will explain the characteristics of the described figl1 recq4 mutants in Arabidopsis and the possible differences of these with FIGL and/or RECQ4 knockdowns.

\section{Pollen tetrads were not present in inoculated plants with TRV2-RFQ.}

Since no pollen tetrads were visible in the triple knockdown, we could not use the silencing of QRT2 as a phenotypic marker to select putative silenced pollen grains in plants treated with TRV-RFQ. The QRT2 target sequence in TRV-RFQ was identical to the sequence used to successfully induces pollen tetrads in Chapter 2, so there must be other reasons for the absence of a quartet phenotype in VIGS-treated plants in this experiment. We postulate that orientation of the QRT2 sequence in the insert may not be optimal to induce silencing of the target gene. To show that using VIGS we can induce a quartet phenotype, we created a VIGS construct in which the QRT2 sequence was cloned in anti-sense orientation (Chapter 2). However, in TRV-RFQ the sequence was cloned into the vector in sense orientation, which may have compromised the silencing efficiency. Also, the insert cloned into TRV2-RFQ was about 2-3 times larger (622 bp) than the previously tested single VIGS constructs that were between $\approx 100-300 \mathrm{bp}$ in length (Chapter 2 and 3). Thus, either insert length, orientation, or both could have decreased or annulled the power of the viral vector to ultimately silence QRT2. As described in Chapter 2, a simultaneous knockdown of REC8-PRD1-OSD1 was not successful, confirming the results obtained here. It suggests that simultaneous knockdown of two or more genes using a single combined VIGS construct is not 
straightforward. Further experiments testing different insert lengths in sense and antisense orientation should be tested to confirm if these two factors limit the stability of the insert in the vector. In addition, TRV2-QRT2 constructs cloned in sense and antisense orientation combined with other experimentally tested VIGS vectors (Chapter 2 and 3 ) should be done to demonstrate its use as a phenotypic pollen marker.

Offspring showing increased recombination frequencies is not recovered in inoculated plants with TRV2-RFQ.

Microscopic observation of DAPI stained meiocytes in Col-O inoculated with TRV2-RFQ confirmed that we could phenocopy the figl1 recq4 mutant phenotype as observed by Fernandes et al., 2017. This essentially shows that recombination frequencies can be increased through VIGS. However, subsequent backcrosses with pollen from silenced flowers of inoculated plants with TRV2-RFQ revealed that we were unable to obtain high-recombinant offspring, while recovery of offspring was reported for figl1 recq4 in Arabidopsis (Fernandes et al., 2017). However, the absence of high recombinant offspring in figl1 recq4 mutant/knockdown backgrounds is not unique to our set-up. For instance, figl1 mutants in rice and pea are completely and exclusively male sterile and hence unable to produce offspring. OsFIGL1 was reported to be essential for pollen viability and anther development (Zhang et al., 2017). In addition, a stop-codon mutation in RECQ4 in pea leads to a severe decrease in pollen viability, and in Psrecq4 -/- mutant hybrids a four-fold reduction in the seed-set (Mieulet et al., 2018). Therefore, the reasons why the absence of figl1 and recq4 leads to infertility or decreased fertility need to be addressed to understand their role in gamete viability.

The null recq4 and/or figl1 mutants may not be viable in Arabidopsis.

The complete male sterility observed in rice and pea figl1 mutants and the reduced fertility of recq4 in pea, raise the intriguing question as to why in Arabidopsis the mutant phenotype is not more extreme with respect to the mutant phenotypes described in crops. Although there is reduction of pollen viability and seed-set in figl1 F1 hybrid and Col-0 but not in figl1 Col-0 in Arabidopsis, both backgrounds were able to set seed and produce viable offspring. As shown in Figure 1, Arabidopsis figl1 mutants commonly have mutations in the second half of the gene (Girard et al., 2015). Interestingly all the figl1 mutant alleles in Arabidopsis were altered only in the AAA-ATPase domain, the VPS4 domain, or in the introns immediately upstream or downstream of these domains. Yet, no mutant allele was found for the FRBD domain that is required for the interaction of FIGL1 with RAD51, and possibly with DMC1 (Figure 1). The figl1 recq4 hybrid used in Fernandes et al., 2017 carried two different figl1 mutant alleles, only affected in its AAA-ATPase domain. A possible explanation can be that a truncated protein with an active FRBD domain can still regulate RAD51/DMC1 dynamics/numbers. In other words, the Arabidopsis mutants may not be proper null-mutants as they exhibit residual activity. In our experimental set-up, contrary to the Arabidopsis figl1 and figl1 recq4 mutants 
described in the literature, we might (likely) have caused a complete absence of FIGL1, which may have led to complete pollen abortion of silenced cells and hence, absence of high recombinant offspring.

Additional problems affecting pollen viability may arise if (also) RECQ4 was silenced in inoculated plants with TRV2-RFQ. RECQ4 suppresses HR in somatic and meiotic cells, but is also involved in the resolution of recombination intermediates and plays an important role during DNA replication and DNA repair (Hartung, Suer and Puchta, 2007; Schröpfer et al., 2014; Séguéla-Arnaud et al., 2017). Here, as in the case of figl1 mutants, mutants of RECQ4 producing high recombinant viable gametes, may not be null-mutants. The recq4 mutant used in Fernandes et al 2017, was previously characterized by Hartung et al., 2007 and was shown to have a T-DNA insertion only affecting the transcription of its helicase domain. However, a transcript from exon 16 to exon 25 in the recq4A mutant is still produced, which corresponds to the RECQ4-Nterminal region. This region in RECQ4 is essential for its role in HR and DNA repair and it may be crucial during the subsequent mitotic divisions of the pollen after meiosis. Therefore, a complete knockdown of RECQ4 is also expected to cause problems during DNA repair, DNA replication, and suppression of HR in somatic cells. These complex pleiotropic effects may very well result in complete pollen abortion.

RECQ4 and FIGL1 may be pleiotropic genes and their function in both somatic and meiotic HR and possibly, pollen development should be further studied in null and/or complete knockdown backgrounds. This could be done using RNAi or CRISPr/cas9 in Arabidopsis and subsequently characterize and compare the phenotype obtained to the recq4 and figl1 mutants described to date. The power of VIGS to silence negative regulators of class II COs can be still assessed. For instance, a modification of the VIGS construct design to use shorter insert sequences in both, sense and antisense orientation should also be tested to discern if differences in insert length and orientation leads to higher penetrance of the silencing phenotype. This may lead also to the possibility to simultaneously knock down two or more genes in meiosis, coupled with a pollen phenotypic marker like the knockdown of $Q R T$ genes. Finally, to better evaluate the use of VIGS to eventually generate high-recombinant offspring, one could test new VIGS constructs for instance in a Fluorescent Tester Line background (FTLs) (Fernandes et al., 2017). 


\section{Materials and methods.}

\section{Plant material.}

F1 hybrids, Col-0 and $m s$ Ler were grown under standard conditions in growth chambers with $16 \mathrm{H} / 8 \mathrm{H}$ light cycle, $21^{\circ} / 18^{\circ} \mathrm{C}$ and $60 \%-50 \% \mathrm{RH}$. BC1 treated and wild-type offspring were grown under semi-controlled greenhouse conditions.

2. Insert design and identification of potential off-targets using SGN VIGS.

Each possible insert in a VIGS vector will lead to the production of molecules of 21-24 nucleotides that trigger the silencing of targets with shared homology (see Table 1). Because the sequences are so short, they can share homology not only with the target gene but also with other genes, causing off-target silencing. To identify possible offtargets when the selected RECQ4, FIGL1 and QRT2 sequences were used in a VIGS vector, we used the online SGN-VIGS (Fernandez-Pozo et al., 2015). SGN VIGS predicts how many molecules of 21-24 nucleotides can be produced for one sequence. We established the length of the molecules for the prediction analysis to 21 base-pairs (hereafter called $21 \mathrm{n}$-mere), as it is the minimum length required to induce silencing. This prediction was done for all the possible $21 \mathrm{n}$-meres produced by each sequence, allowing zero and one mismatches with the target sequence (see Table 1). From this analysis we learned that only one $21 \mathrm{n}$-mere molecule produced by the insert sequence cloned in the TRV2-RFQ construct could potentially target the locus AT2G38770.1.

Table 1. Prediction of the number of $21 \mathrm{n}$-meres produced by each insert sequence. The total number of $21 \mathrm{n}$-meres that could target each gene allowing 0 mis-matches and 1-mis-match is indicated for TRV2-RECQ4_1, TRV2-RECQ4_1, TRV2-FIGL1 and TRV2-RFQ.

Number of mis-matches allowed (0/1) for prediction of 21-nmeresTargets: RECQ4A/B FIGL1 and QRT2

\begin{tabular}{|c|c|c|c|c|c|c|c|c|}
\hline $\begin{array}{c}N^{\circ} \text { of } n \text {-meres } \\
\text { predicted for } \\
\text { each } \\
\text { construct }\end{array}$ & $\begin{array}{c}0 \\
R E C Q 4 A\end{array}$ & $\begin{array}{c}0 \\
R E C Q 4 B\end{array}$ & $\begin{array}{c}1 \\
R E C Q 4 A\end{array}$ & $\begin{array}{c}1 \\
R E C Q 4 B\end{array}$ & $\begin{array}{c}0 \\
\text { FIGL1 }\end{array}$ & $\begin{array}{c}1 \\
\text { FIGL1 }\end{array}$ & $\begin{array}{c}0 \\
\text { QRT2 }\end{array}$ & $\begin{array}{c}1 \\
\text { QRT2 }\end{array}$ \\
\hline $\begin{array}{l}\text { TRV2- } \\
\text { RECQ4_1 }\end{array}$ & 174 & 10 & 174 & 35 & 0 & 0 & 0 & 0 \\
\hline $\begin{array}{c}\text { TRV2- } \\
\text { RECQ4_1 }\end{array}$ & 145 & 2 & 145 & 42 & 0 & 0 & 0 & 0 \\
\hline TRV2-FIGL1 & 0 & 0 & 0 & 0 & 148 & 148 & 0 & 0 \\
\hline TRV2-RFQ & 220 & 12 & 222 & 35 & 149 & 151 & 180 & 181 \\
\hline
\end{tabular}




\title{
3. Plasmid construction.
}

RNA from Col-0 flower buds was extracted by using the QUIAGEN RNAeasy mini Kit ${ }^{\circledR}$ (cat. $n^{\circ}$. 74104). cDNA was synthesized by using the RevertAid First Strand cDNA synthesis $\mathrm{Kit}^{\circledR}$ (cat. $\left.n^{\circ} \mathrm{K} 1621\right)$ from ThermoFisher Scientific. A FIGL1 cDNA region of 169 bp was PCR-amplified by using a FIGL1_F primer 5'CATCTAGAACCACCGGAGTTTCTGAATG -3 ' and a FIGL1_R primer 5'CAGAATTCTAGAAGACGAAGCCCAAGGA -3' and clone in antisense orientation into TRV2 ( $p Y L 156$ ) to generate the TRV2-FIGL1 vector. The restriction sites Xbal and $E C O R I$ were added to the forward and the reverse primer respectively and inserted into the PCR product. TRV2-RECQ4_1 were created by amplifying a cDNA region of $194 \mathrm{bp}$ using RECQ4_F1 primer 5'- CACTCGAGGGATGGAATGGGCTGAACAA-3' and the RECQ4_R1 primer 5'-CAGGTACCTCCCCATTGGCTCACACAAT-3'. For TRV2RECQ4_2 cDNA region of 166 bp was amplified by using the RECQ4_F2 primer 5'CACTCGAGCGCTCTGATCTGTGGAGGAA-3' and a RECQ4_R2 primer 5'CAGGTACCATGCTCGGAATTCAGCTCCT - 3 '. In both cases, the restriction sties Xhol and $K p n l$ were respectively added to the forward and reverse primer sequences and inserted in the PCR product leading to both inserts cloned in antisense orientation. Final TRV2 vectors were verified by sequencing and independently cloned into Agrobacterium tumefaciens GV3101 (pMP90) strain.

Construction of the TRV2-RFQ was done using a commercialized synthesized fragment containing sequences of (169 bp) RECQ4, (240 bp) FIGL1 and (197 bp) QRT2 and delivered in a commercial vector (Thermofisher scientific). The CDS used to clone the insert containing the triple target RECQ4 (yellow)-FIGL1 (red)-QRT2 (black) is shown below. Sequences highlighted in pink indicate the restriction sites inserted in the fragment.

\begin{abstract}
CATCTAGATGGTATTCTGAAGCAGAAATTTCCCAACATTCCCGTGTTAGCTTTAACAGCTACAGCA ACAGCCAGTGTGAAAGAAGATGTGGTACAAGCCCTTGGCCTTGTTAACTGTGTCGTGTTCCGGC AAAGTTTTAATCGTCCAAATCTATGGTATTCTGTTGTTCCCAAGACAAAAAAGTGTCTGGAAGACA TCGACAAATTTATTAAGGAAAACCATTTTGATGAGTGTGGAATTATATATTGCACCATGGACCACC GGAGTTTCTGAATGTGAAGGAGGAAGGAGAAACGGAGACGCCGTGTTGGAGAAAGGAAGTGGA TGAGAACTTGAAGCGTCTCCAATCGCTGCTCTTCGGCGCCGACAAATTCCTGGAGAAATCTGATT TCTCTTCTGCTCAGATCCTTGGGCTTCGTCTTCTACAGGATCCGCGGTACAAGTGAGCAATGTGT TGTACAAAAACATACAAGGGACGAGCTCAAGACCCATAGCTGTGAAATTTGTATGCAGCAAGA ACATTCCATGTCGAGGAATATCAATGCAAAACGTTAAACTGGTCGATCAAACTCAACAAGATGT ATCCAAAGCTTCTTGCTCCAATGTGAAGTTGGACACCCGAGGAAATGTCAGGTACC
\end{abstract}

4. Agrobacterium-incubation and inoculation protocol.

Consult Chapter 5 of this thesis for a detailed description of this protocol. 
5. Selection of flower material and $\mathrm{BC} 1$ generation.

Systematic checks of every flower that opened starting three w.p.i was done for $22 \mathrm{~F} 1$ hybrid plants for five consecutive days. To evaluate the pollen phenotype, we selected one anther per flower and stained it in a phenol-free Alexander solution as described in Peterson et al., 2010 (Peterson, Slovin and Chen, 2010). Flowers producing aborted pollen were used to pollinate $m s$ Ler plants. Uninfected $\mathrm{F} 1$ hybrids were used as control plants and in a control cross with $m s$ Ler to produce wild-type $\mathrm{BC} 1$ offspring. The genotyping data-set obtained for the wild-type and treated $\mathrm{BC} 1$ population can be retrieved using the following link: http://doi.org/10.4121/uuid:33c2cd57-c041-4b2cb15b-736b2451e7e1

\section{Cytology}

Flower buds of $\mathrm{F} 1$ hybrids were fixed in a mixed of 3:1 EtOH-Acetic acid respectively at 18 d.p.i. One day after fixation, the flower buds were washed twice with $70 \% \mathrm{EtOH}$ and store at $-4{ }^{\circ} \mathrm{C}$. Meiotic chromosome spreads were made following protocols previously described (Ross, Fransz and Jones, 1996; Armstrong, 2013) stained with DAPI and then analysed with a Zeiss microscope equipped with appropriate epifluorescence optics. Pollen staining was done using the staining solution protocol described in Peterson et al.,2010. 


\section{References.}

1. Armstrong, S. (2013) 'Spreading and fluorescence in situ hybridization of male and female meiocyte chromosomes from Arabidopsis thaliana for cytogenetical analysis.', in Pawlowski, W. P., Grelon, M., and Armstrong, S. (eds) Plant Meiosis: Methods and Protocols. Methods in Molecular biology. New York: Springer Science + Business Media, pp. 3-11. doi: 10.1007/978-1-62703-333-6_1.

2. Becker, A. (2013) Virus-Induced Gene Silencing. Methods in Molecular Biology. Edited by A. Becker. Humana Press. doi: 10.1007/978-1-62703-278-0.

3. Berchowitz, L. E. et al. (2007) 'The role of AtMUS81 in interference-insensitive crossovers in A. thaliana', PLoS Genetics, 3(8), pp. 1355-1364. doi: 10.1371/journal.pgen.0030132.

4. Copenhaver, G. P., Housworth, E. A. and Stahl, F. W. (2002) 'Crossover interference in Arabidopsis', Genetics, 160(4), pp. 1631-1639. doi: 10.1086/376610.

5. Crismani, W. et al. (2012) 'FANCM limits meiotic crossovers', Science, 336(6088), pp. 15881590. doi: $10.1126 /$ science. 1220381.

6. Falque, M. et al. (2009) 'Two types of meiotic crossovers coexist in maize', The Plant Cell, 21(12), pp. 3915-3925. doi: 10.1105/tpc.109.071514.

7. Fernandes, J. B. et al. (2017) 'Unleashing meiotic crossovers in hybrid plants', Proceedings of the National Academy of Sciences, 115(10), pp. 2431-2436. doi: 10.1073/pnas.1713078114.

8. Fernandes, J. B. et al. (2018) 'FIGL1 and its novel partner FLIP form a conserved complex that regulates homologous recombination.', PLoS genetics, 14(4), p. e1007317. doi: 10.1371/journal.pgen.1007317.

9. Fernandez-Pozo, N. et al. (2015) 'The SGN VIGS tool: User-friendly software to design virus-induced gene silencing (VIGS) Constructs for functional genomics', Molecular Plant, 8(3), pp. 486-488. doi: 10.1016/j.molp.2014.11.024.

10. Girard, C. et al. (2015) 'AAA-ATPase FIDGETIN-LIKE 1 and helicase FANCM antagonize meiotic crossovers by distinct mechanisms.', PLoS Genetics, 11(7), pp. 1-22. doi: 10.1371/journal.pgen.1005369.

11. Hartung, F., Suer, S. and Puchta, H. (2007) 'Two closely related RecQ helicases have antagonistic roles in homologous recombination and DNA repair in Arabidopsis thaliana', Proceedings of the National Academy of Sciences, 104(47), pp. 18836-18841. doi: 10.1073/pnas.0705998104.

12. Higgins, J. D. et al. (2008) 'AtMSH5 partners AtMSH4 in the class I meiotic crossover pathway in Arabidopsis thaliana, but is not required for synapsis', The Plant Journal, 55(1), pp. 28-39. doi: 10.1111/j.1365-313X.2008.03470.x.

13. Knoll, A. and Puchta, H. (2011) 'The role of DNA helicases and their interaction partners in genome stability and meiotic recombination in plants', Journal of Experimental Botany, 62(5), pp. 1565-1579. doi: 10.1093/jxb/erq357.

14. Korte, A. and Farlow, A. (2013) 'The advantages and limitations of trait analysis with GWAS: A review', Plant Methods, 9(29), pp. 1-9. doi: 10.1186/1746-4811-9-29.

15. Lambing, C., Franklin, F. C. H. and Wang, C.-J. R. (2017) 'Understanding and manipulating meiotic recombination in plants.', Plant Physiology, 173(3), pp. 1530-1542. doi: 10.1104/pp.16.01530.

16. Lu, X. et al. (2008) 'The Arabidopsis MutS homolog AtMSH5 is required for normal meiosis', Cell Research, 18(5), pp. 589-599. doi: 10.1038/cr.2008.44.

17. Lynn, A., Soucek, R. and Börner, G. V. (2007) 'ZMM proteins during meiosis: Crossover artists at work', Chromosome Research, 15(5), pp. 591-605. doi: 10.1007/s10577-007-1150- 
1.

18. Mannuss, A. et al. (2010) 'RAD5A, RECQ4A, and MUS81 have specific functions in homologous recombination and define different pathways of DNA Repair in Arabidopsis thaliana', The Plant Cell, 22(10), pp. 3318-3330. doi: 10.1105/tpc.110.078568.

19. Mercier, R. et al. (2005) 'Two meiotic crossover classes cohabit in Arabidopsis: One is dependent on MER3, whereas the other one is not', Current Biology, 15(8), pp. 692-701. doi: 10.1016/j.cub.2005.02.056.

20. Mieulet, D. et al. (2018) 'Unleashing meiotic crossovers in crops', Nature plants ., 4(12), pp 1010-1016. doi 10.1038/s41477-018-0311-x

21. Peterson, R., Slovin, J. P. and Chen, C. (2010) 'A simplified method for differential staining of aborted and non-aborted pollen grains', International Journal of Plant Biology, 1(2), pp. 66-69. doi: 10.4081/pb.2010.e13.

22. Ross, K. J., Fransz, P. and Jones, G. H. (1996) 'A light microscopic atlas of meiosis in Arabidopsis thaliana.', Chromosome Research, 4(7), pp. 507-516. doi: 10.1007/BF02261778.

23. Schröpfer, S. et al. (2014) 'Defining the roles of the N-terminal region and the helicase activity of RECQ4A in DNA repair and homologous recombination in Arabidopsis', Nucleic Acids Research, 42(3), pp. 1684-1697. doi: 10.1093/nar/gkt1004.

24. Séguéla-Arnaud, M. et al. (2015) 'Multiple mechanisms limit meiotic crossovers: TOP3a and two BLM homologs antagonize crossovers in parallel to FANCM', Proceedings of the National Academy of Sciences, 112(15), pp. 4713-4718. doi: 10.1073/pnas.1423107112.

25. Séguéla-Arnaud, M. et al. (2017) 'RMI1 and TOP3a limit meiotic CO formation through their C-terminal domains', Nucleic Acids Research, 45(4), pp. 1860-1871. doi: 10.1093/nar/gkw1210.

26. Senthil-Kumar, M. and Mysore, K. S. (2011) 'New dimensions for VIGS in plant functional genomics', Trends in Plant Science. Elsevier Ltd, 16(12), pp. 656-665. doi: 10.1016/j.tplants.2011.08.006.

27. Serra, H. et al. (2018) 'Massive crossover elevation via combination of $\mathrm{HEI} 10$ and recq4a recq $4 b$ during Arabidopsis meiosis.', Proceedings of the National Academy of Sciences, 115(10), pp. 2437-2442. doi: 10.1073/pnas.1713071115.

28. Wijnker, E. and de Jong, H. (2008) 'Managing meiotic recombination in plant breeding', Trends in Plant Science, 13(12), pp. 640-646. doi: 10.1016/j.tplants.2008.09.004.

29. Zhang, P. et al. (2017) 'The rice AAA-ATPase OsFIGNL1 Is essential for male meiosis', Frontiers in Plant Science, 8(1639), pp. 1-17. doi: 10.3389/fpls.2017.01639. 
To the will of pursuing the unknown.

\author{
"You let your feet run wild \\ time has come as we all oh, go down \\ yeah but for the fall oh, my \\ do you dare to look him right in the eyes?"
}

Way down we go, Kaleo.

"Las ideas no duran mucho.

Hay que hacer algo con ellas".

Ramon y Cajal. 


\section{Chapter 5}

\section{A protocol for the downregulation of meiotic}

genes using virus-induced gene silencing (VIGS) in Arabidopsis thaliana.

\section{Authors.}

Vanesa Calvo-Baltanás¹, Arp Schnittger², Hans de Jong¹, Erik Wijnker¹. Author affiliations.

1) Laboratory of Genetics, Wageningen University \& Research, P.O. Box 16 6700 AA Wageningen, The Netherlands.

2) Department of Developmental Biology, University of Hamburg, Institute for plant sciences and microbiology, 22609 Hamburg, Germany. 


\section{Abstract.}

Virus-Induced Gene Silencing (VIGS) is a transient silencing technique that exploits the immune system response to a viral infection to silence endogenous genes in several plant species, including the model organism Arabidopsis thaliana. VIGS can be used to downregulate the expression of meiotic genes in Arabidopsis thaliana, for example to change meiotic inheritance. We here present a protocol that describes all necessary steps to successfully target meiotic genes of choice in $A$. thaliana using the TRV-based VIGS system (Tobacco rattle virus). Because the induced phenotype by TRV virus in Arabidopsis is present for a limited period, we briefly detail how to time the moment of viral inoculation with plant development to target a gene in meiosis. We also explain how to verify meiotic phenotypes in inoculated plants through cytological approaches in pollen mother cells and pollen development. Lastly, we provide a working guideline to recover offspring from with VIGS treated plants and to timely sample flower buds for meiotic chromosome spreads.

\section{Introduction.}

Meiosis is the motor that fuels the generation of new allele combinations. There is therefore a great interest in the possible breeding applications that may arise from the modification of meiosis to change genetic inheritance (Wijnker and de Jong, 2008; Lambing, Franklin and Wang, 2017). The interest in inducing phenotypes that alter meiotic crossover formation concerns various different phenotypes. For instance, the induction of $2 n$ gametes using abiotic treatments like cold or heat shock (De Storme, Copenhaver and Geelen, 2012; Wang et al., 2017) or the use of chemicals like colchicine or nitrous oxide (Forster et al., 2007; Younis, Hwang and Lim, 2014) have been described as efficient approaches to promote diploid gamete production, and the generation of polyploid offspring. Increasing recombination frequencies may increase the chance of recovering crossover recombination events between closely linked genes and can promote the generation of mapping populations with high mapping resolution (Fernandes et al., 2017; Serra et al., 2018). Lastly, the reduction of crossover frequencies brings forth the possibility of reverse breeding, that in Arabidopsis proved highly valuable in the generation of mapping populations based on chromosome substitution lines (Wijnen et al., 2018). The modification of this processes at will can therefore serve to develop and improve breeding applications.

Virus-induced gene silencing (VIGS) is a transient silencing approach used in a large variety of crops to downregulate endogenous genes (Senthil-Kumar and Mysore, 2011). VIGS has been first used to downregulate genes in meiosis in wheat (Bennypaul et al., 2012; Bhullar et al., 2014). Later, we showed that VIGS could be used to downregulate recombination frequencies in meiosis in Arabidopsis thaliana for reverse breeding. In addition, it was shown that silenced gametes can give rise to viable offspring, and therewith could be used for breeding applications (Chapter 3). 

induced gene silencing (VIGS) in Arabidopsis thaliana

Furthermore, tetraploid offspring was obtained after inducing a second meiotic restitution in Arabidopsis thaliana (Chapter 2). A major benefit of VIGS-for-meiosis is that it can rapidly and efficiently silence a target gene in the host plant. In Arabidopsis, inoculated plants with a VIGS vector display a phenotype at 10 days post-inoculation (Burch-Smith, 2006). A second benefit of VIGS is that renders all Arabidopsis offspring transgene-free. This overcomes the problem of plants harboring dominantly acting transgenes or recessive mutations to modulate meiosis. On the other hand, VIGS is not-fully penetrant and is also transient, which means that in Arabidopsis, induced meiotic phenotypes (e.g. pollen abortion derived from the absence of crossover formation, enlarged pollen grains corresponding to diploid gamete production) will only be partially and temporarily present.

To ensure that a meiotic phenotype is induced during flower development (and meiosis) it is necessary to understand how the silencing mechanism works and how it is timely regulated. VIGS exploits the posttranscriptional gene silencing (PTGS) response to downregulate a host gene, which is triggered by the presence of viral dsRNAs in the plant. (Ratcliff, Martin-Hernandez and Baulcombe, 2001; Susi et al., 2004; Burch-Smith, 2006). One of the preferred VIGS systems used in Arabidopsis relies on the use of the modified the Tobacco Rattle Virus (TRV). The TRV VIGS system is a bipartite virus consisting of two components: TRV RNA2 and TRV RNA1 (hereafter TRV2 and TRV1). TRV2 encodes for non-structural- and coat proteins and it has been engineered to allow cloning of an insert homologous to the target gene. TRV1 is essential for viral replication and movement (Liu et al., 2002; Burch-Smith, 2006; Senthil-Kumar and Mysore, 2014). TRV1 and TRV2 are cloned into separate Agrobacterium tumefaciens strains and administered to the plant by co-inoculation (Burch-Smith et al., 2004). Once both bacteria transform leaf cells and the vectors are delivered to the plant, TRV2 and TRV1 generate an active RNA virus that will produce dsRNAs intermediates during its autonomous replication (Burch-Smith et al., 2004; Burch-Smith, Miller and Dinesh-Kumar, 2006; Padmanabhan and Dinesh-Kumar, 2009). The dsRNAs activate The RISC complex, after which it is cleaved by DICERlike proteins into 21-24-nucleotide siRNA, molecules, that are, in turn, loaded into ARGONAUTE as single-stranded siRNA (Xie et al., 2004; Becker and Lange, 2009; Deng et al., 2013). The siRNAs then serve as guide molecule for the RISC complex to find complementary, also single-stranded RNA molecules, which are then recognized as putative viral transcripts, triggering their degradation (Hamilton and Baulcombe, 1999; Susi et al., 2004). The insert (cDNA plant sequence) cloned into TRV2, will lead to the degradation of endogenous mRNA of the plant host, hence silencing the target gene in the plant. Yet, mRNAs will only be silenced if viral siRNAs are present and transported in the plant, which is the case when the virus actively replicates and accumulates within its host (Becker and Lange, 2009). Because RNA virus like TRV usually have a limited life-span in the host (Ratcliff, Martin-Hernandez and Baulcombe, 

induced gene silencing (VIGS) in Arabidopsis thaliana

2001; Bernacki et al., 2010), its activity must be precisely timed with the moment in which meiosis takes place.

This chapter explains how to use VIGS to modify meiotic genes in Arabidopsis thaliana. We first describe the possible applications of the method to continue with the possible limitations and, by taking this into account, ensuring silencing in meiosis. The experimental set-up will be explained thereafter. In the experimental set-up we first introduce the steps for generating a VIGS construct. The next two steps in the experimental set-up consist of the experimental design and protocol execution: plant inoculation with TRV, phenotypic validation and finally, offspring generation.

\section{Applications of the method.}

A wide variety of meiotic genes can be targeted in Arabidopsis using VIGS (see Chapters 2-4). Meiotic phenotypes were obtained for the downregulation of meiotic recombination meiosis after silencing $\mathrm{MSH} 5$ (Chapter 3) and the upregulation of recombination by targeting FIGL/RECQ4 (Chapter 4). Furthermore, the knockdown of genes involved in sister chromatid cohesion (REC8) and DSBs and CO formation (PRD1, SDS) induced a pollen abortion phenotype. The knockdown of $Q R T 1$ and $Q R T 2$ leads to the formation of pollen tetrads while the silencing of OSD1 induced $2 \mathrm{n}$ pollen production (Chapter 2). The described approach so far has not clearly shown the possibility of knocking-down two endogenous genes in meiosis simultaneously. Therefore, we suggest to use this protocol for targeting genes that lead to distinct pollen phenotypes as compared to wildtype. The presence of a distinctly recognizable pollen phenotype will facilitate assessing as to whether VIGS silencing is successful during the experiments.

\subsection{Plant functional gene analysis.}

VIGS has been previously used to simultaneously knock down redundantly acting genes that share a conserved sequence (Burch-Smith et al 2004). This alleviates the need for the laborious generation of double, triple or quadruple mutants. Albeit not specifically tested in meiosis, it is likely that the same strategy could be applied to meiotic genes. VIGS also allows to conduct fast reverse genetic screens (Burch-Smith, 2006; Senthil-Kumar, Lee and Mysore, 2013). Genes for which no knockouts are available, but for which a meiotic mutant phenotype is expected, may be good targets for VIGS. After phenotypic validation, other methods like CRISPR/cas9 or RNAi may be employed to enable the study of a more stable phenotype. Furthermore, VIGS could be used to study phenotypes in meiosis that otherwise would cause embryolethality in a mutant or knockout background. 

induced gene silencing (VIGS) in Arabidopsis thaliana

\subsection{VIGS for reverse breeding.}

The downregulation of meiotic recombination may lead to interesting applications in Arabidopsis, like reverse breeding, which produces homozygous lines from a heterozygous plant that can be thereafter used to reconstitute the initial hybrid or as a mapping population (Wijnker et al., 2012; Wijnen et al., 2018). The downregulation of recombination increases the frequency of non-recombinant chromosomes segregating to spores. When spores from a heterozygous plant carrying non-recombinant chromosomes are regenerated as haploid plants by crossing to a haloid inducer line (Ravi and Chan, 2010), the resulting offspring will carry combinations of nonrecombinant chromosomes of the parental hybrid. Haploid Arabidopsis plants can set seed, and as such give rise to doubled-haploid offspring $(\mathrm{DH})$. If the starting hybrid was an $\mathrm{F} 1$, this procedure gives rise to chromosome substitution lines that are powerful mapping tools for, among others, QTL detection and the study of epistasis (Wijnen et al., 2018). In addition, the initial heterozygous can be reconstituted as an F1 hybrid when two complementing homozygous lines are crossed (Chapter 3).

\subsection{VIGS to generate polyploids.}

VIGS has also been applied in Arabidopsis to produce polyploids (Chapter 2). This has been achieved by targeting OSD1, a protein required on the onset of meiosis I to meiosis II. The silencing of OSD1 leads to a second meiotic restitution (SDR) and production of both, female and male diploid gametes. If plants are left to set seed, polyploid offspring can be recovered from VIGS inoculated plants (Chapter 2). The main benefit of using VIGS to induce a meiotic restitution is that it will produce eutetraploid (balanced) offspring. This a main advantage compared to other methods like colchicine, which leads to large rates of aneuploid offspring. Furthermore, colchicine, is a hazardous chemical that can cause changes in chromosome structure at meiosis (Rodriguez et al., 2001) and several abnormalities in mitosis, such as anaphase bridges or micronuclei formation in higher rates than in wild-type mitosis (Kundu and Ray, 2017).

\subsection{VIGS and tetrad analysis.}

QRT genes encode for pectinestarases which are involved in the separation of the haploid spores after meiosis (Preuss, Rhee and Davis, 1994; Rhee and Somerville, 1998; Francis, Lam and Copenhaver, 2006). Mutants of these genes produce pollen tetrads as a result of unresolved haploid pollen grains (Preuss, Rhee and Davis, 1994; Rhee and Somerville, 1998; Copenhaver, Keith and Preuss, 2000). Furthermore, VIGS constructs for Arabidopsis also induced qrt phenotypes in Col-O and Ler inoculated plants (Chapter 2). These could allow tetrad analysis in genetic backgrounds that are not qrt mutants (Berchowitz et al., 2007; Wijnker et al., 2013). Conversely, it would also be possible to apply VIGS to lines in a qrt mutant background, like the Fluorescent 
A protocol for the downregulation of meiotic genes using virusinduced gene silencing (VIGS) in Arabidopsis thaliana

Tester Lines (FTLs) (Francis et al., 2007). These are lines in which recombination frequencies can be monitored using lines with fluorescent reporter proteins that are expressed from a pollen specific promoter. If genes are expected to affect recombination frequencies, these changes may be observed directly in pollen through altered segregation ratios between fluorescent markers (Fernandes et al., 2017; Séguéla-Arnaud et al., 2017).

\section{How to ensure silencing in meiosis: plant age, growing conditions and genotype limits VIGS efficiency.}

VIGS, as any other method has inherent limitations that can greatly affect its potential to induce silencing in meiosis. The time in which the VIGS vector remains stable and the rate of viral accumulation within the host depends on various factors (Burch-Smith et al., 2004; Fu et al., 2006; Becker, 2013; Bilichak and Kovalchuk, 2017). In the following section we will focus only on growing conditions, plant age and genotype as main factors that in our experience can greatly affect the penetrance of the silencing phenotype. Furthermore, we provide general rules for VIGS that should be later implemented in the experimental-setup.

\subsection{Plant age and developmental stage for agroinoculation.}

Plant age determines VIGS efficiency, that decreases if older plants are inoculated. The virus has the potential to spread systemically in the host plant, virtually affecting all the tissues (Burch-Smith et al., 2004; Bennypaul et al., 2012; Becker, 2013) but as plants grow older, they become less susceptible to the silencing (Burch-Smith et al., 2006; Bilichak and Kovalchuk, 2017). This is possibly related to changes in the architecture of the leaf, increasing plasmodesmata complexity, and differences in plasmodesmata aperture that interfere with the systematic spread of the virus and viral siRNAs (Kalantidis et al., 2008; Becker, 2013; Heinlein, 2015; Pyott and Molnar, 2015). For instance, Burch-Smith et al., 2006 reported that silencing efficiency is higher if seedlings at 2 to 3 leaf-stage are inoculated compared to seedlings that are at 4 to 5 leaf-stage. Therefore, for successful viral accumulation and transmission of siRNAs in the host plant, Agrobacterium-inoculation should preferentially happen in early plant development stages (Burch-Smith et al., 2006; Bilichak and Kovalchuk, 2017). However, not only the timely inoculation of a plant is key to success. The duration and penetrance of VIGS-induced phenotypes is correlated with the stability of the virus and the generated siRNAs to accumulate within the host plant. When the virus spreads and replicates, dsRNAs and siRNAs ultimately accumulate and move within a short and long-range in the plant (Becker and Lange, 2009). In our experience, this may relate to a peak in silencing efficiency that last for $\approx 3-5$ days, happening $\approx 18$ days postinoculation (Chapters 2-4) (figure 1). If a meiotic gene is to be targeted, this peak in silencing efficiency must coincide with a moment in which in the host plant meiosis is 
ongoing. For this reason, the time between agroinoculation and the onset of flower development (bolting) should be $\approx 18$ days, (figure 1). Moreover, due to the fact VIGS efficiency is higher when young plants are agroinoculated (seedlings at 4-5 leaf stage as upper stage limit) (Burch-Smith et al., 2006; Bilichak and Kovalchuk, 2017), accessions that take a long time to flower (or even require vernalization) cannot be targeted using the current VIGS approach. Therefore, the current technique is best suited for early flowering accessions with flowering times similar to Columbia or Landsberg erecta (Chapter 2).

\subsection{Growing conditions.}

Growing conditions can be decomposed in three main parameters: temperature, light intensity and relative humidity $(\mathrm{RH})$. Optimum values for these three parameters promote healthy plant growth and high VIGS efficiency. Growing conditions that are set at $21^{\circ} \mathrm{C}$ (Burch-Smith, 2006) or $21 / 18^{\circ} \mathrm{C}$ (Chapter 3), $16 \mathrm{H} / 8 \mathrm{H}$ light cycle and $60 \%$ $\mathrm{RH}$ have been reported to induce efficient silencing of $P D S$ and of meiotic genes. On the other hand, higher temperatures of about $24^{\circ} \mathrm{C}-26{ }^{\circ} \mathrm{C}$ decrease the efficiency of VIGS (Bilichak and Kovalchuk, 2017). Furthermore, different accessions growing in the same environment may develop at different rates. Therefore, it is important to thoroughly monitor the three main developmental stages for VIGS: 4-5 leaf stage, bolting time and flowering time under the chosen growing conditions. In addition, the susceptibility to VIGS under chosen growing conditions may vary among accessions (Chapter 2). To test this, it is recommended to silence PHYTOENE DESATURASE (PDS). PDS silencing causes obvious photobleaching symptoms (see figure 3 ) and it is regularly used as positive phenotypic control in VIGS assays (See Protocol in BurchSmith et al., 2006). Hence, penetrance of the PDS silencing phenotype is a good proxy to address susceptibility to the silencing under the chosen growing conditions (BurchSmith et al 2006). For Col-0 and Ler plants growing under described conditions, the silencing efficiency of $P D S$ is $100 \%$ and $65 \%$ of the total inoculated plants respectively (Chapter 2 and 3; Burch-smith et al. 2006). Thus, a test with TRV2-PDS is always advised to estimate how many plants will be used later in the experimental set-up, based on the penetrance of the silencing phenotype under the local growing conditions (Figure 2).

\subsection{Genotype susceptibility.}

Different genotypes display variable susceptibility to VIGS (Chapter 2 and 3, BurchSmith et al., 2004; Senthil-Kumar and Mysore, 2011), and this may prohibit the use of VIGS to specific genotypes. This was shown for a random sample of 17 early flowering accessions in which PDS was downregulated. Out of these 17 accessions, in one Mib-22- silencing of $P D S$ did not lead to a silencing phenotype (Chapter 2). Therefore, a first test to VIGS susceptibility is best done by inoculating the target accession with TRV2-PDS (Burch-Smith et al., 2006). The percentage of the plants showing 
photobleaching can be used as an estimation of VIGS efficiency for that accession (Burch-smith et al. 2006; Chapter 2). Additionally, silencing of PDS in the accession of choice can be compared with inoculated Col-0 plants that can be used as positive control. Monitoring the number of plants showing a PDS phenotype is recommended between 10-20 days (Burch-Smith et al., 2006; Chapter 2). If the accession does not show photobleaching, unlike positive control lines, it is likely that the accession is insensitive to the VIGS, using a TRV-based system.

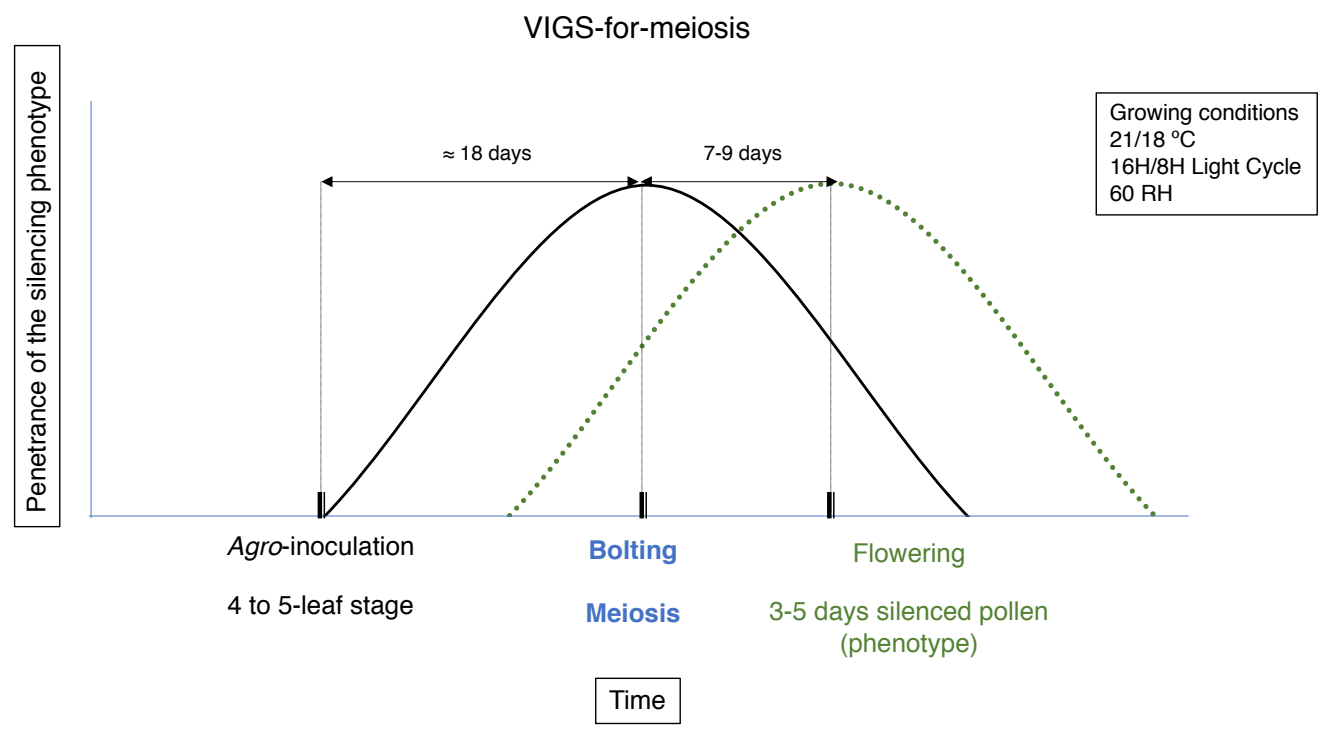

Figure 1. Correlation between viral accumulation and penetrance of the silencing phenotype over time after agroinoculation with a TRV VIGS vector in Arabidopsis plants. At first the virus replicates exponentially, spreading in the plant to reach a maximum level. After few weeks, the total count of virus starts to decline (Burch-Smith et al., 2004; Bernacki et al., 2010). This phenomenon is graphically represented by the black curve. To ensure efficient silencing, seedlings at 4 to 5 leaf-stage (as oldest stage) should be inoculated (Burch-Smith et al., 2006). The highest penetrance of the silencing phenotype ( $y$-axis) will be then correlated to the highest presence of virus and/or derived siRNAs in the host plant. To efficiently downregulate a meiotic gene, the silencing must affect the meiocytes by the time the plant is bolting. We observed this peak happens about 18 days d.p.i, although differences between accessions used should be experimentally assessed (Chapter 2). If the period from meiosis to mature pollen production is about 7-9 days in Arabidopsis (Liu, De Storme and Geelen, 2018), the manifestation of the silencing phenotype in the pollen will be then shifted for 7-9 days, represented by the green doted curve.

\section{Experimental set-up: "VIGS-for-meiosis".}

The experimental set-up can only be planned and executed once the main developmental stages (4-5 leaf stage, bolting and flowering time) and susceptibility of the chosen accession to VIGS (test to silence PDS) are known under the local growing 
conditions (see figure 1). The experimental set-up is divided in three separate procedures (figure 2). The first and second procedures are mainly preparational. The first one comprises the transformation of Agrobacterium strains with a TRV VIGS construct. Rather than providing a step-by-step protocol for these procedures, we will provide general guidelines and refer to relevant protocols that have been published elsewhere. Secondly, we will give an estimation of the amount of plants that should be used in the experiment. Finally, a detailed procedure will be described concerning the actual execution of the VIGS protocol to target meiotic genes (procedure 3).

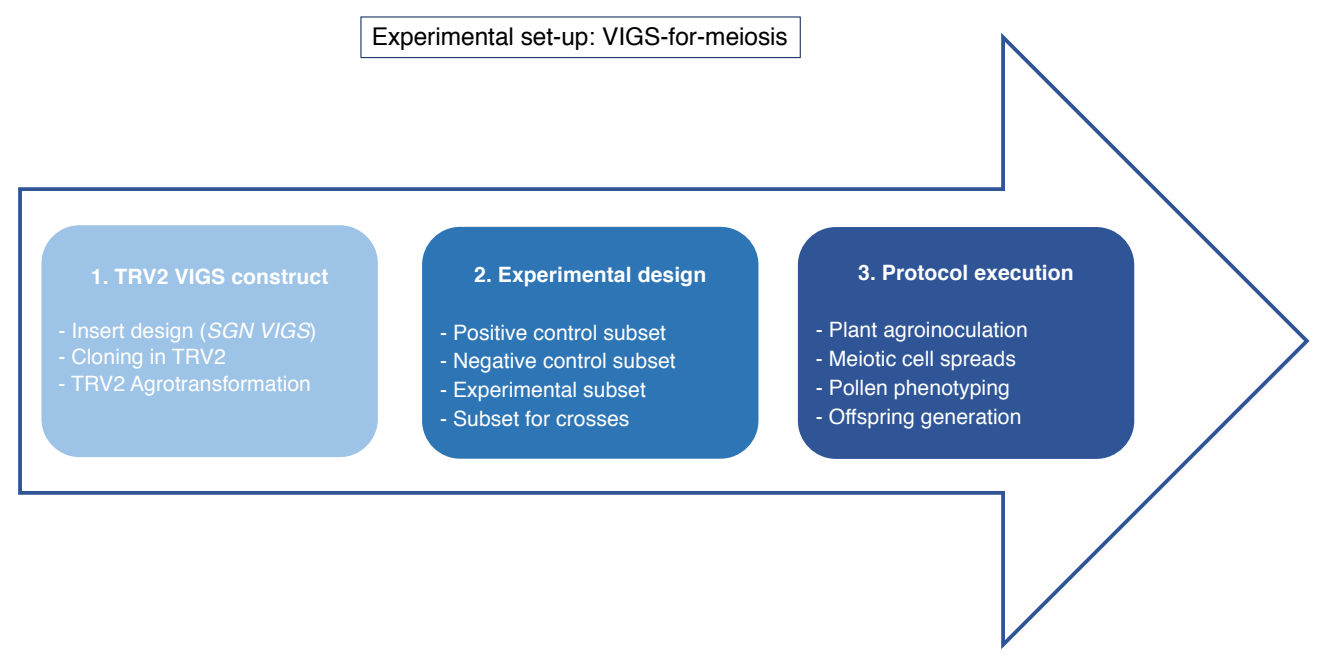

Figure 2. Schematic representation of the experimental set-up of VIGS for meiosis. Step 1 is the generation of the VIGS construct and consequent transformation of Agrobacterium. Step 2 describes the experimental design based on the number of plants required for the VIGS experiment that is then executed in step 3.

\subsection{Generation of a TRV2 VIGS construct.}

Several protocols have explained the importance of promoting insert stability in a VIGS vector to ensure efficient silencing over time (Burch-Smith et al., 2004; Wu, Lingling and Goggin, 2011; Ding et al., 2018). In the following sections we provide the general guidelines for insert design focused on a TRV2-based VIGS system for meiosis in Arabidopsis, as other species and VIGS systems present different specifications. For the vector construction, we briefly explain the procedure and refer, whenever necessary, to protocols in place for the execution of the most relevant steps during the cloning procedure. 


\section{A. Insert design.}

Adequate insert sequence design is the primary step to guarantee efficient silencing. Several studies have described the importance of maintaining a specific insert length, composition and orientation to promote insert stability in the viral vector (Burch-Smith, Miller and Dinesh-Kumar, 2006; Liu and Page, 2008; Senthil-Kumar and Mysore, 2011, 2014; Becker, 2013). Here, we encourage to use the online tool SGN VIGS (http://vigs.solgenomics.net) to identify possible off-targets when designing an insert. SGN VIGS helps to design an insert with the appropriate length and nucleotide composition characteristics to ensure stability in the TRV VIGS system for Arabidopsis.

Identification of off-targets.

Inserts of target sequences in TRV2 lead to the generation of different viral siRNAs of 21-24 nucleotides that, in case of shared sequence with other genes, may potentially trigger unintended silencing of off-targets (Senthil-Kumar and Mysore, 2011; Fernandez-Pozo et al., 2015). The insert should be designed based on exon sequences which are preferentially present in all the splicing variants of the transcribed mRNA (Ruiz, Voinnet and Baulcombe, 1998) but it should not contain sequence homology to other targets. To minimize the risk of off-targets, the online software SGN VIGS can predict whether the 21-24 nucleotide-siRNAs generated from an insert are likely to silence unwanted targets (Fernandez-Pozo et al., 2015). Using this tool one can identify unique genic regions to minimize the chance that a VIGS construct silences genes other than the target sequence.

Insert orientation and length.

Several studies recommended that all inserts are preferably cloned in antisense orientation into the TRV2 vector to increase the penetrance of the silencing phenotype (Pflieger et al., 2008; Senthil-Kumar and Mysore, 2011; Becker, 2013). Yet, inserts in sense orientation can also induce silencing in meiosis (Chapter 2 and 3). Furthermore, insert length, is generally accepted in the range between 200-350 bp long. Bigger inserts may limit viral movement, and if this, occurs, is likely that the virus will remove the insert from its genome (Burch-Smith et al., 2004, 2006; Senthil-Kumar and Mysore, 2011). It has also been observed that inserts of $\approx 100-300$ bp can promote effective silencing in meiosis (Chapter 2-4)

B. Cloning and Agrobacterium transformation with TRV2 vector.

The cloning of a VIGS vector requires that first, primer sequences are designed for the insert sequence to later obtain a PCR fragment. The PCR fragment will be then cloned into TRV2 following a restriction-ligation reaction, generating the final VIGS construct 

induced gene silencing (VIGS) in Arabidopsis thaliana

that will be used to transformed Agrobacterium tumefaciens. The steps and references to the appropriate protocols to achieve this are listed below.

Vectors:

- TRV VIGS system has been described in (Liu et al., 2002). TRV2 empty vector (pYL156) can be retrieved from stock number CD3-1040, Tair accession 4515440204 and TRV1 (pYL192) can be retrieved from stock number, CD3-1039, Tair accession 4515440203

- TRV2-PDS has been described in Burch-Smith et al 2004 (Burch-Smith, 2006)

- TRV2-QRT1 and TRV2-QRT2 have been described in Chapter 2.

\section{Primers:}

The insert that will be cloned into TRV2 is the PCR product amplified using primers designed on the insert sequence chosen for the target gene (See A, insert design). Because the cloning method is followed by a digestion-ligation procedure, two restriction sites that are present in the TRV2 backbone vector should be incorporated in the insert. To include the restriction sites in the insert, they must be first added to the sequences of both primer pairs that will be used for subsequent PCR amplification (see example below). This sequence should be added to 5 'end which will then allow the insertion of the restriction site in the PCR fragment. We recommend that to each primer of the pair, two different restriction sites are used. This will later on increase ligation efficiency of the insert to the TRV2 empty vector.

Fw - 5' - CA - Restriction site - Primer sequence - 3'

Rv -5' - CA-Restriction site - Primer sequence - 3'

\section{Cloning Procedure:}

\section{RNA extraction}

RNA extraction should be done from flower buds of Arabidopsis thaliana. This will ensure that the RNA extracted that will serve thereafter as a template for CDNA synthesis corresponds to genes that are expressed in meiosis. We recommend that RNA is extracted using RNAeasy Plant mini kit (QIAGEN).

2. cDNA and PCR amplification

We recommend that CDNA synthesis is done using Transcription First Strand cDNA Synthesis Kit (Roche). Following cDNA synthesis, primers designed based on the insert sequence should be used for PCR amplification.

3. Insert Digestion and ligation to TRV2 backbone vector The insert is first cloned into the pTRV2 vector (pYL156) following a classical digestion-ligation protocol described in https://www.addgene.org/protocols/subcloning/ and in https://international.neb.com/Protocols/0001/01/01/dna-ligation-with-t4-dnaligase-m0202.For digestion and ligation, we recommend to used FastDigest enzymes (Thermo Scientific) and T4 ligase (Thermo Scientific). 

induced gene silencing (VIGS) in Arabidopsis thaliana

4. E.coli transformation with TRV2-insert

TRV2-insert and TRV1 should be cloned into E.coli following a classical transformation protocol by heat shock (Froger and Hall, 2007). Upon plasmid extraction, the TRV2insert should be verified by sequencing using the primer Fw_TRV2

\section{GATGGACATTGTTACTCAAGGAAG}

\section{Agrobacterium transformation with TRV2-insert}

After sequencing verification, Agrobacterium tumefaciens (GV3101) transformation with TRV2 can be done following a heat-shock protocol (http://www.dnacloning.com/agrobacterium). An extra verification step by colony PCR is required before preparing glycerol stocks.

\subsection{Experimental design.}

The experimental design describes the number of plants that will be used in the experiment. This should be planned in advance based on the ultimate goal of the experiment. For instance, silencing validation through meiotic chromosome spreads or pollen phenotype will require a different design than if the goal is to produce progeny using silenced gametes (figure 2 and 3 ). The experimental design always includes positive controls, negative controls and experimental subset plants. These three groups are the absolute minimum to keep a strict control and validation of the phenotype. Alternatively, a subset of plants to generate offspring can be also included.

1. Positive controls:

- Silencing of $P D S$ guarantees that agroinoculation was optimal and that silencing of the meiotic target could be expected. The number of positive controls must be calculated based on the efficiency of the PDS silencing test (see section 3: Growing conditions, plant age and genotype limits VIGS efficiency). If $100 \%$ of the inoculated plants with TRV2-PDS had previously displayed photobleaching symptoms, then the number of positive controls should be at least three. However, if the penetrance of the PDS silencing phenotype for the accession used is lower, this number should increase accordingly to guarantee that at least three plants show photobleaching symptoms.

- Silencing of $Q R T$ genes induces pollen tetrads. $Q R T$ genes can be knocked down in a separate subset of plants to verify that the time between inoculation and bolting was optimum to induce silencing in the gametes. To validate this, we recommend to grow a subset control plants and inoculate them with TRV2-QRT. Although a subset of plants inoculated with TRV2-QRT is not strictly necessary, we recommend that it is included in each VIGS experiment, especially if a new VIGS construct will be used. For this, we establish an absolute 

induced gene silencing (VIGS) in Arabidopsis thaliana

minimum of 3 plants, increasing accordingly if the susceptibility of the accession is low.

2. Negative controls: This is a subset of uninfected plants that are grown together with inoculated TRV2 plants and used for comparison. They must always be grown alongside the positive controls and the experimental subset. The number of negative controls should never be lower than three.

3. Experimental subset: These plants will be inoculated with TRV2 to silence a meiotic gene. This subset will be used to validate the meiotic phenotype using cytological approaches for meiocytes and/or pollen and will be used to generate offspring if needed.

- Plants used for meiocyte cytology: The number of agroinoculated plants used to collect inflorescences for spread slides of pollen mother cells should be at least 40 . This number is calculated considering that the phenotype is not fully penetrant (not all the flower buds will be affected) and that only one flower bud out of each collected inflorescence, is expected to have anthers with pollen mother cells at a certain meiotic stage. We further recommend to include 10 extra plants and leave them to flower to monitor when a pollen phenotype appear in those. If the time in which flower buds were harvested occurred 7-9 days before that, it should be possible to observe a phenotype in meiotic chromosome spreads.

- Plant subset for pollen phenotyping: It has been observed that at least $50 \%$ of Col-0 and Ler plants in which meiosis has been targeted show a phenotype (Chapter 2 and 3). Accordingly, and based previous experiments (Chapter 2-4) the number of plants recommended are not lower than 40 plants per target, in which at least 20 are expected to be silenced for about 3-5 days (Chapters 3 and 4). This number is sufficient to validate an induced meiotic phenotype and generate offspring. In Col-0 and F1 Ler-col hybrids this occurs about 25 d.p.i (Chapter 2-4)

4. Plants used in crosses (non-inoculated). If the generation of offspring is the aim of the experiment at least three mother plants should be included in the experimental set-up. Flowering time of the mother plants should coincide with the observation of the silencing in the pollen in the subset of treated plants. Alternatively, the pollen can be cold-stored to use it later in crosses (Bou Daher, Chebli and Geitmann, 2009). Secondly, when plants are to be crossed after treatment with VIGS, it is important that the crossing partners have flowers at the same time in which the silencing phenotype is present in inoculated plants. We have for example noticed that GFP-tailswap, the haploid inducer line for Arabidopsis (Ravi and Chan, 2010), flowers relatively late in 

induced gene silencing (VIGS) in Arabidopsis thaliana

comparison to the Col-0 wild-type from which it was derived. Therefore, such lines must be sown earlier to enable cross-pollination.

\section{3. Protocol execution.}

The following section details the actual procedure for plant agroinoculation with a VIGS construct. For a successful protocol execution, planification of the dates for agroincubation and agroinoculation coordinated to plant growth is crucial (figure 1 and figure 3). Because agroinoculation should occur around 18 days before bolting, bolting time after germination should be known in advance (figure 1), and it should correspond to seedlings in the $4-5$ leaf at latter stage.

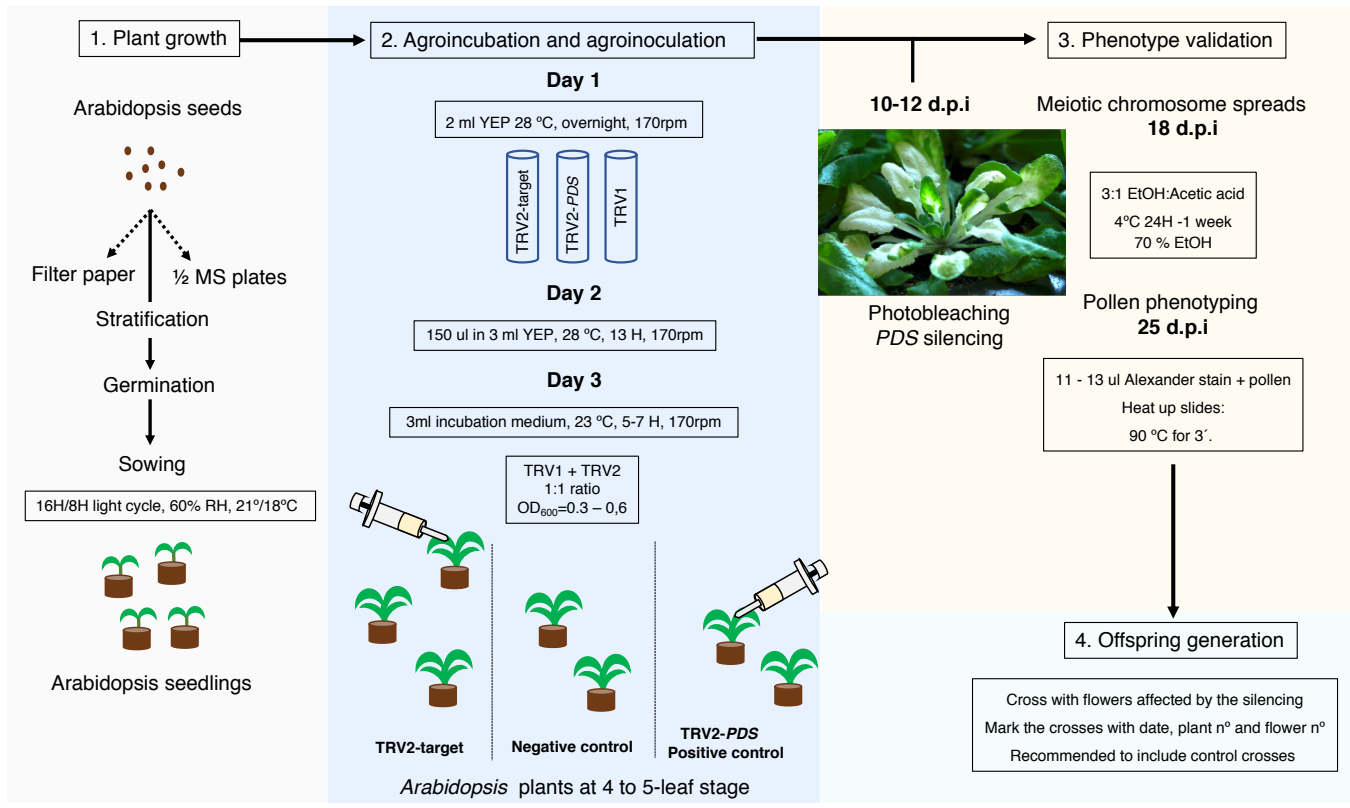

Figure 3: Schematic representation of the main steps of the procedure of VIGS-for-meiosis. The 1st step corresponds to plant growth which includes, stratification, germination and sowing of the Arabidopsis seeds. The $2^{\text {nd }}$ step explains the protocol of agroincubation and agroinfiltration with the TRV2 and TRV1 vectors spaced over three consecutive days. The $3^{\text {rd }}$ step explains the necessary methods and important time-points to evaluate the phenotype in meiocytes and mature pollen in open flowers. The phenotype caused by the silencing of $P D S$ is expected to be seen at $\approx 10$ d.p.i (BurchSmith et al., 2006). The $4^{\text {th }}$ step briefly indicates how to generate offspring following a systematic approach using flowers producing pollen grains showing a silencing phenotype. 
1. Plant growth.

1. Book a growth chamber.

2. Sterilize the seeds. The selected number of seeds (including positive and negative controls) can be sterilized following the hydrochloric fumigation protocol described in Wijnker et al., 2014 or other preferred method of seed sterilization.

3. Stratify the seeds. Seeds should remain at $4^{\circ} \mathrm{C}$ for up to a maximum of 7 days to make sure that all the plants will germinate at once. In this step, seeds can already be placed in $1 / 2$ MS plates (see recipes) or on imbibed filter paper.

4. Let the seeds germinate. Transfer $1 / 2$ MS plates or petri dishes with filter paper containing the seeds to a growth chamber. If seeds were stratified on $1 / 2 \mathrm{MS}$ plates, place these vertically in a growth chamber, to prevent roots from tangle into the agar. Seedlings can grow for up to 7 days on plates before transferring to pots. If seeds were stratified on filter paper, it is recommended to transfer them to pots one day after germination to avoid rooting in the filter paper.

5. Transfer the seedlings to pots. To avoid plant stress during growth, plants are best grown in individual pots.

6. Label each individual plant with an identification number. To assess pollen phenotypes, unique labels and numbers allow identification of each plant.

7. Before the agroincubation and agroinfiltration:

a. Book a shaker that allows to incubate at $28^{\circ} \mathrm{C}$ and $23^{\circ} \mathrm{C}$ for the selected dates in which the agroincubation steps -prior to the agroinfiltration- will take place (see figure 3 ).

b. Prepare YEP liquid medium and incubation medium (see recipes).

c. Inoculate bacteria to LB plates with appropriate antibiotic.

1. Prepare LB agar plates (see recipes). LB plates should contain 70 $\mu \mathrm{g} / \mathrm{ml}$ gentamicin and $70 \mu \mathrm{g} / \mathrm{ml}$ kanamycin as antibiotic selection.

2. Grow Agrobacterium tumefaciens transformed with TRV2 and TRV1 in LB agar plates. The bacteria should be grown from the glycerol stocks previously prepared (see VIGS vector cloning procedure) at $28^{\circ} \mathrm{C}$ for 2 days.

2. Agroincubation and agroinfiltration (3-day protocol) (modified from Nimchuck et al 2000 and Burch-smith et al. 2006).

8. Day 1: agroincubation overnight in $2 \mathrm{ml}$ YEP culture.

a. Label separate crystal tubes with the names of each TRV2 vector to be used. For each TRV2 vector culture that will be prepared, one TRV1 vector culture should be grown to later mix these in a proportion of 1:1 (see figure 3). 
b. Prepare bacterial cultures. Select a single colony (TRV2insert, TRV2-PDS, -TRV2-QRT2, TRV1) or inoculate the bacteria directly from glycerol stocks in separate crystal tubes containing $2 \mathrm{ml}$ of YEP medium $+50 \mu \mathrm{g} / \mathrm{ml}$ gentamicin and $50 \mu \mathrm{g} / \mathrm{ml}$ kanamycin.

c. Incubate overnight at $28^{\circ} \mathrm{C}$ and shaking at $170 \mathrm{rpm}$. Make sure the tubes are tilted during shaking to allow air flow in the culture.

9. Day 2: agroincubation overnight in $3 \mathrm{ml} \mathrm{YEP}$ culture.

a. Take out the bacteria cultures from the incubator. Bacteria can be stored at $4^{\circ} \mathrm{C}$ until the next incubation step.

b. Prepare bacteria cultures. Take $150 \mu \mathrm{l}$ of each culture and inoculate in separate crystal tubes containing $3 \mathrm{ml}$ of YEP medium $+50 \mu \mathrm{g} / \mathrm{ml}$ gentamicin and $50 \mu \mathrm{g} / \mathrm{ml} \mathrm{kanamycin}$.

c. Incubate for no longer than $13 \mathrm{H}$-to avoid bacteria overgrowth- at $28^{\circ} \mathrm{C}$ and shaking at $170 \mathrm{rpm}$. Make sure that tubes are tilted to allow air flow in the culture.

10. Day 3. agroincubation in $3 \mathrm{ml}$ incubation medium and agroinfiltration.

a. Take out the bacteria cultures from the incubator.

b. Transfer the cultures into a $15 \mathrm{ml}$ plastic tube.

c. Pellet the bacteria at $5000 \mathrm{rpm}$ for 8 minutes at RT. After centrifugation, a bacterial pellet should be clearly visible.

d. Discard the supernatant and resuspend the bacteria using a pipette after adding $3 \mathrm{ml}$ incubation medium (see recipes).

e. Incubate at $23^{\circ} \mathrm{C}$ for 5-7 hours, shaking at $170 \mathrm{rpm}$.

f. Pellet the bacteria at $5000 \mathrm{rpm}$ for 8 minutes at RT.

g. Prepare inoculation medium (see recipes). This can be done during centrifugation. The inoculation medium should always be prepared fresh.

h. Discard the supernatant and use a pipette to resuspend the bacteria in $3 \mathrm{ml}$ inoculation medium.

i. Measure the bacteria concentration $(O D=600)$ using a spectrophotometer.

j. Adjust the final bacteria concentration of each culture to $\mathrm{OD}_{600}=0.3-0.6$ by diluting with inoculation medium if necessary. All the cultures should have the same final concentration.

k. Mix each TRV2 culture with a TRV1 culture in a 1:1 ratio. 

induced gene silencing (VIGS) in Arabidopsis thaliana

I. Agroinfiltrate the leaves of each plant from the underside using a $20 \mathrm{ml}$ needleless syringe. Infiltration of every leaf in the plant can boost the silencing by increasing the inoculum in the plant.

\section{Phenotype validation}

3.a. Chromosome spreads of meiocytes (agroinoculated and control plants).

11. Fix flower buds $\approx 18$ d.p.i with Carnoy (see recipes) and store at $4^{\circ} \mathrm{C}$. Flower buds may need to be cut out of the rosette.

12. Change the fixative to $70 \% \mathrm{EtOH}$ after $24 \mathrm{~h}$. and store at $4^{\circ} \mathrm{C}$

13. Meiotic chromosome spreads can be done following previous protocols described in (Ross, Fransz and Jones, 1996; Armstrong, 2013)

3.b. Pollen phenotyping in agroinoculated and control plants.

14. Evaluate the pollen phenotype to verify silencing in meiosis. Evaluate open flowers from the moment in which the first flower opens in each plant (see the step 26.a and 26.b for specification in "offspring generation").

15. Monitor the phenotype caused by the silencing over time. Identify each plant showing a pollen phenotype and check in those plants open flowers for about 3-5 days from the moment in which the first silenced flower appeared.

16. Label a microscope glass slide. The slides should be identified with date, plant $\mathrm{n}^{\circ}$ and flower $\mathrm{n}^{\circ}$.

17. Add a drop of 11-13 $\mu$ l Alexander staining solution to each slide (see recipes).

18. Sample the pollen. Detach an anther of the selected flower and shake it over the slide repeatedly to ensure that the pollen is released into the staining solution. The whole anther can also be stained and kept in the slide.

19. Cover with $18 \times 18$ cover-slip (only if $11-13$ ul of solution are used). Bigger sized cover-slips will squeeze the pollen grains and smaller sizes may not cover the sample.

20. Heat up the slides for a better color differentiation between aborted and viable pollen. Slides should be heated on a heating plate up to $90^{\circ} \mathrm{C}$ for 3 minutes.

21. Visualize the pollen with a microscope using bright field.

22. Slides can be stored at $4^{\circ} \mathrm{C}$ for 1 month to guarantee their integrity.

4. Offspring generation using flowers producing pollen showing a silencing phenotype

23. Select open-flowers.

a. Self-fertilized flowers must be kept in the plant and labeled with a colored-thread or other desired identification method. Flowers producing wild-type like pollen can be removed from the plant. 
b. Flowers used for cross-pollination can be removed from the inoculated plant.

24. Cross with selected flowers. To maximize the number of offspring obtained, only 1 anther should be used to pollinate 1 mother flower. All the silenced flowers should be used for the cross.

25. Make control crosses. The negative controls can be used as pollen donor plants as long as they are the same genotype as the inoculated plants used for crosses. This control step is recommended if offspring will be genotyped or sequenced.

26. Label the crosses. To retrieve the information of the flower used for the cross and compare it to the pollen slide made, the crosses should be labeled with the date, plant $\mathrm{n}^{\circ}$ and flower number.

\section{Troubleshooting, materials and recipes.}

\subsection{Troubleshooting.}

\begin{tabular}{|c|c|c|c|}
\hline Step & Problem & Possible reason & Solution \\
\hline $\begin{array}{l}9 \& \\
10\end{array}$ & $\begin{array}{l}\text { No or slow bacteria growth after } \\
\text { the } 1^{\text {st }} \text { or } 2^{\text {nd }} \text { incubation process }\end{array}$ & $\begin{array}{l}\text {-High glycerol concentration } \\
\text {-Using old stocks/plates } \\
\text {-To high antibiotic concentration }\end{array}$ & $\begin{array}{l}\text { - Make sure the final glycerol } \\
\text { concentration is }<40 \% \\
\text { - Prepare fresh glycerol } \\
\text { stocks } / \text { plates } \\
\text { - Reduce the antibiotic } \\
\text { concentration to } 50 \mathrm{\mu g} / \mathrm{ml}\end{array}$ \\
\hline 10 & Overgrown/dead bacteria & $\begin{array}{l}\text {-Too long incubation time } \\
\text {-Contamination in the cultures }\end{array}$ & $\begin{array}{l}\text {-Repeat incubation step } 9 \\
\text {-Prepare new incubation } \\
\text { /inoculation medium }\end{array}$ \\
\hline $\begin{array}{l}13 \& \\
14\end{array}$ & $\begin{array}{l}\text { Absence of meiotic and/or } \\
\text { pollen phenotype }\end{array}$ & $\begin{array}{l}\text { - Not enough inoculum } \\
\text { - Wrong inoculation time } \\
\text { - VIGS construct is not stable/efficient } \\
\text { - Not enough plants used for sampling }\end{array}$ & $\begin{array}{l}\text {-Make sure all the leaves are } \\
\text { always infiltrated } \\
\text {-Test inoculation with TRV2- } \\
\text { AtQRT at three different } \\
\text { timepoints. Then, the right } \\
\text { moment of inoculation can be } \\
\text { inferred from the appearance } \\
\text { of pollen tetrads. } \\
\text { - Colony PCR to make sure } \\
\text { that the TRV2 vector cloned in } \\
\text { Agrobacterium contains the } \\
\text { complete insert. } \\
\text {-Clone new insert (follow insert } \\
\text { design guidelines explained in } \\
\text { this protocol). } \\
\text { - If the penetrance of the } \\
\text { silencing phenotype is low, } \\
\text { sample size (flower buds/open } \\
\text { flowers) should be enlarged. }\end{array}$ \\
\hline 24 & $\begin{array}{l}\text { Crosses do not lead to viable } \\
\text { offspring }\end{array}$ & $\begin{array}{l}\text { - Flowers exhibiting high pollen abortion rates } \\
\text { were used in the crosses }\end{array}$ & $\begin{array}{l}\text { - Select only one anther and } \\
\text { use it to pollinate only flower at } \\
\text { the time, this will increase the } \\
\text { amount of viable pollen that } \\
\text { can fertilize the ovule. } \\
\text { - Select viable pollen under the } \\
\text { microscope to pollinate with. }\end{array}$ \\
\hline
\end{tabular}




\subsection{Materials.}

\section{Reagents}

1. Agrobacterium tumefaciens incubation and inoculation.

Incubation mediums (YEP, LB and antibiotics)

Yeast Extract (Duchefa Biochemie, cat.no Y1333.1000)

Peptone (Duchefa Biochemie, cat.no. P1328.1000)

Sodium Chloride (NaCL, Duchefa Biochemie, cat.no. S0520.500)

Kanamycin sulfphate (Amresco, cat.no. 0408-EU-10G)

Gentamicin sulphate (Duchefa Biochemie, cat.no. G0124.0001)

ddH2O.

Infiltration medium and inoculation medium

Dipotassium phosphate $\left(\mathrm{K}_{2} \mathrm{HPO}_{4}\right.$; cat.no 7758-11-4)

Potassium dihydrogen phosphate $\left(\mathrm{KH}_{2} \mathrm{PO}_{4}\right.$; Merck, cat.no. 7778-77-0)

Ammonium sulfate $\left(\left(\mathrm{NH}_{4}\right)_{2} \mathrm{SO}_{4}\right.$

Trisodium Citrate Dyhidrate (Na citrate, Duchefa Biochemie, cat.no. S0521.5000)

Trisodium Citrate Dyhidrate (Na citrate, Duchefa Biochemie, cat.no. S0521.5000)

Magensium sulphate hepatydrate $\left(\mathrm{MgSO}_{4}\right.$; AppliChem, cat.no. 10034-99-8)

D-Glucose monohydrate (Duchefa Biochemie, cat.no. G0802.5000)

D-Fructose (Duchefa Biochemie, cat.no. 57-48-7)

Glycerol, 99\% (Sigma, cat.no. G5516-500ML)

Morpholineethanesulfonic acid (MES, Sigma-Aldrich, cat.no 4432-31-9)

$\mathrm{HCL}$

Acetosyringone (Sigma-Aldrich, cat.no. D134406-5G)

Murashige and Skoog (MS) medium basal, powder (Duchefa Biochemie, cat.no.

M0221.0050)

Potassium hydroxide (KOH, Merck 1310-58-3)

Sucrose crystalized (Duchefa Biochemie, cat.no. S0809.5000).

2. Alexander staining

Ethanol absolute (EtOH, VWR chemicals, cat.no. 20821.330

Malachite green (Sigma, cat.no. M9015-25G)

Acid fuchsin (Sigma, cat.no F8129-25G)

Orange G (Sigma, cat.no. 0-1625)

Acetic acid (Sigma, cat.no. F8129-25G)

Glycerol (Duchefa Biochemie, cat.no. G1345.5000)

3. Plant germination

Agar powder food grade (AppliChem, cat.no A0917,1000) 
Murashige and Skoog (MS) + vitamins, powder (Duchefa Biochemie, cat.no.

M0221.0050)

$\mathrm{KOH}$

\section{Equipment}

Soil suitable to grow Arabidopsis thaliana

Pots.

Filter paper

Petri dishes

Glass tubes

Incubator-shaker in which temperature can be adjusted between $23^{\circ} \mathrm{C}$ and $28^{\mathrm{a}} \mathrm{C}$

Sterilized pipettes tips

Pipettes

Table centrifuge

Pipettes (Eppendorf)

Falcon tubes $14 \mathrm{ml}$ (Starstedt)

Schott bottles $250 \mathrm{ml}$

Disposable syringes sterile (Luer connection, $20 \mathrm{ml}$ tub. NEOLAB E1500)

Gloves (Klüver and Schulz)

Glass beaker, $30 \mathrm{ml}$

Autoclave

Microscope slides (Starfrost, cat. $n^{\circ}$ V511371077FKA)

Glass coverslips 18X18 (Th Geyer, cat.n ${ }^{\circ}$ WC/07695023/00000)

S1Climate chamber (CLF climatics Grobanks)

S1 Laboratory suitable for Agro-transformation

Flow cabinet

Fume hood

Stainless steel fine-point tweezers (Dumont, cat. $n^{\circ} 2-1033$, NEOLAB)

Dissecting microscope

Binocular

Air Pump

Labels

Filtropur BT50 500ml 22um (83.1823.101 Starstedt)

Square Petri dishes (Greiner, cat. $n^{\circ} 688161$ )

Round Petri dishes

Heating plate 

induced gene silencing (VIGS) in Arabidopsis thaliana

\subsection{Recipes.}

LB medium (solid and liquid medium)

Per $1 \mathrm{~L}$ of $\mathrm{ddH}_{2} \mathrm{O}: 15 \mathrm{~g}$ of peptone, $5 \mathrm{~g}$ of yeast extract, $10 \mathrm{~g}$ of $\mathrm{NaCl}$ and adjust the $\mathrm{pH}$ to 7,2 . To prepare solid medium, add $15 \mathrm{~g}$ of agar. Autoclave for 20 minutes at $120 \mathrm{C}^{\circ}$. YEP (solid and liquid medium)

Per $1 \mathrm{~L}$ of $\mathrm{ddH}_{2} \mathrm{O}: 10 \mathrm{~g}$ of peptone, $10 \mathrm{~g}$ of yeast extract, $5 \mathrm{~g}$ of NaCl and adjust the $\mathrm{pH}$ to 7,2 . To prepare solid YEP medium, at $15 \mathrm{~g}$ of agar. The medium must be always autoclaved.

$1 / 2$ MS Medium (solid)

Per $1 \mathrm{~L}$ of $\mathrm{ddH}_{2} \mathrm{O}$ : In $900 \mathrm{ml}$ of $\mathrm{ddH}_{2} \mathrm{O} 2,2 \mathrm{~g}$ of MS + vitamins, $10 \mathrm{~g}$ of sucrose. Adjust $\mathrm{pH}$ to 5.7-5.8 with $1 \mathrm{M} \mathrm{KOH}$. Add $8 \mathrm{~g}$ of agar and adjust to a final volume of $1 \mathrm{~L}$. Agrobacterium tumefaciens GV3101::TRV-RNA1 and TRV-RNA2 glycerol stocks Inoculate a PCR-verified colony in $4 \mathrm{ml}$ of LB medium with $50 \mu \mathrm{g} / \mathrm{ml}$ gentamicin and 50 $\mu \mathrm{g} / \mathrm{ml}$ kanamycin concentration and incubate at $28^{\circ} \mathrm{C}$ for a maximum 13 hours.

Glycerol stocks must be prepared in a $40 \%$ final glycerol concentration using $99 \%$ sterilized pure glycerol.

Phenol-free Alexander staining (Peterson, Slovin and Chen, 2010)

Mix $10 \mathrm{ml}$ of $95 \% \mathrm{EtOH}, 1 \mathrm{ml}$ of malachite green (1\% solution in $95 \% \mathrm{EtOH}$ ), $50 \mathrm{ml}$ of ddH2O, $25 \mathrm{ml}$ of glycerol $99 \%, 5 \mathrm{ml}$ of acid fuchsin (1\% solution in ddH2O), $0,5 \mathrm{ml}$ of orange $\mathrm{G}(1 \%$ solution in $\mathrm{ddH} 2 \mathrm{O})$ and $4 \mathrm{ml}$ of acetic acid. After homogenization, add $\mathrm{ddH} 2 \mathrm{O}$ to a total volume of $100 \mathrm{ml}$ and store at RT in the dark.

Incubation medium (Adapted from Nimchuk et al. 2000)

Per 1 liter of $\mathrm{ddH}_{2} \mathrm{O}: 10,5 \mathrm{~g} \mathrm{~K}_{2} \mathrm{HPO}, 4,5 \mathrm{~g} \mathrm{KH}{ }_{2} \mathrm{PO} 4,1 \mathrm{~g}\left(\mathrm{NH}_{4}\right)_{2} \mathrm{SO}_{4}, 0,5 \mathrm{~g}$ Sodium citrate, $1 \mathrm{mM} \mathrm{MgSO}_{4}, 1 \mathrm{~g}$ Glucose, $1 \mathrm{~g}$ Fructose, $4 \mathrm{ml}$ Glycerol, $1,95 \mathrm{~g} \mathrm{MES}$ and adjust the $\mathrm{pH}$ to 5,6 using $\mathrm{HCL}$ and sterilize by filtration using a $22 \mu \mathrm{m}$ filter pore. Add $50 \mathrm{ug} / \mathrm{ml}$ of acetosyringone and store in the dark at $4^{\circ} \mathrm{C}$.

Inoculation medium (Adapted from Nimchuk et al. 2000)

Per 1 liter of $\mathrm{ddH}_{2} \mathrm{O}: 1,95 \mathrm{~g}$ MES and 2,04g MS.

Fixation solution for flower buds

In a $50 \mathrm{ml}$ falcon tube, mix in 3:1 proportion 99\% EtOH:Acetic acid. Wash and replace after $24 \mathrm{H}$ with $70 \% \mathrm{EtOH}$. 


\section{References.}

1. Armstrong, S. (2013) 'Spreading and fluorescence in situ hybridization of male and female meiocyte chromosomes from Arabidopsis thaliana for cytogenetical analysis.', in Pawlowski, W. P., Grelon, M., and Armstrong, S. (eds) Plant Meiosis: Methods and Protocols. Methods in Molecular biology. New York: Springer Science + Business Media, pp. 3-11. doi: 10.1007/978-1-62703-333-6_1.

2. Becker, A. (2013) Virus-Induced Gene Silencing. Methods in Molecular Biology. Edited by A. Becker. Humana Press. doi: 10.1007/978-1-62703-278-0.

3. Becker, A. and Lange, M. (2009) 'VIGS - genomics goes functional', Trends in Plant Science, 15(1), pp. 1-4. doi: 10.1016/j.tplants.2009.09.002.

4. Bennypaul, H. S. et al. (2012) 'Virus-induced gene silencing (VIGS) of genes expressed in root, leaf, and meiotic tissues of wheat.', Functional and Integrative Genomics, 12(1), pp. 143-156. doi: 10.1007/s10142-011-0245-0.

5. Berchowitz, L. E. et al. (2007) 'The role of AtMUS81 in interference-insensitive crossovers in A. thaliana', PLoS Genetics, 3(8), pp. 1355-1364. doi: 10.1371/journal.pgen.0030132.

6. Bernacki, S. et al. (2010) 'Virus-induced gene silencing as a reverse genetics tool to study gene function.', in Hennig, L. and Köhler, C. (eds) Plant Developmental Biology. Methods and Protocols. Zurich: Humana Press, pp. 27-45. doi: 10.1007/978-1-60761-765-5_3.

7. Bhullar, R. et al. (2014) 'Silencing of a metaphase I-specific gene results in a phenotype similar to that of the Pairing homeologous 1 ( Ph1) gene mutations', Proceedings of the National Academy of Sciences, 111(39), pp. 14187-14192. doi: 10.1073/pnas.1416241111.

8. Bilichak, A. and Kovalchuk, I. (2017) 'Increasing a stable transformation efficiency of Arabidopsis by manipulating the endogenous gene expression using Virus-Induced Gene Silencing.', in Kovalchuk, I. (ed.) Plant epigenetics. Methods in Molecular Biology. Boston, MA: Humana Press, pp. 225-236. doi: 10.1007/978-3-319-55520-1.

9. Bou Daher, F., Chebli, Y. and Geitmann, A. (2009) 'Optimization of conditions for germination of cold-stored Arabidopsis thaliana pollen.', Plant cell reports, 28(3), pp. 34757. doi: 10.1007/s00299-008-0647-1.

10. Burch-Smith, T. M. et al. (2004) 'Applications and advantages of virus-induced gene silencing for gene function studies in plants.', Plant Journal, 39(5), pp. 734-746. doi: 10.1111/j.1365-313X.2004.02158.x.

11. Burch-Smith, T. M. et al. (2006) 'Efficient virus-linduced gene silencing in Arabidopsis', Plant Physiology, 142(1), pp. 21-27. doi: 10.1104/pp.106.084624.

12. Burch-Smith, T. M., Miller, J. L. and Dinesh-Kumar, S. P. (2006) 'Delivery of dsRNA into plants by VIGS Methodology', Cold Spring Harbor Protocols, 2006(1), p. pdb.prot4327pdb.prot4327. doi: 10.1101/pdb.prot4327.

13. Copenhaver, G. P., Keith, K. C. and Preuss, D. (2000) 'Tetrad analysis in higher plants. A budding technology.', Plant physiology, 124(1), pp. 7-16. doi: 10.1104/pp.124.1.7.

14. Deng, X. et al. (2013) 'Modification of Tobacco rattle virus RNA1 to serve as a VIGS vector reveals that the $29 \mathrm{~K}$ movement protein is an RNA silencing suppressor of the virus.', Molecular Plant-Microbe Interactions: MPMI, 26(5), pp. 503-14. doi: 10.1094/MPMI-12-120280-R.

15. Ding, X. S. et al. (2018) 'An improved Brome mosaic virus silencing vector: Greater insert stability and more extensive VIGS', Plant Physiology, 176(1), pp. 496-510. doi: 10.1104/pp.17.00905.

16. Fernandes, J. B. et al. (2017) 'Unleashing meiotic crossovers in hybrid plants', Proceedings of the National Academy of Sciences, 115(10), pp. 2431-2436. doi: 

induced gene silencing (VIGS) in Arabidopsis thaliana

\subsection{3/pnas.1713078114.}

17. Fernandez-Pozo, N. et al. (2015) 'The SGN VIGS tool: User-friendly software to design virus-induced gene silencing (VIGS) constructs for functional genomics', Molecular Plant, 8(3), pp. 486-488. doi: 10.1016/j.molp.2014.11.024.

18. Forster, B. P. et al. (2007) 'The resurgence of haploids in higher plants', Trends in Plant Science, 12(8), pp. 368-375. doi: 10.1016/j.tplants.2007.06.007.

19. Francis, K. E. et al. (2007) 'Pollen tetrad-based visual assay for meiotic recombination in Arabidopsis', Proceedings of the National Academy of Sciences, 104(10), pp. 3913-3918. doi: 10.1073/pnas.0608936104.

20. Francis, K. E., Lam, S. Y. and Copenhaver, G. P. (2006) 'Separation of Arabidopsis pollen tetrads is regulated by QUARTET1, a pectin methylesterase gene', Plant Physiology, 142(3), pp. 1004-1013. doi: 10.1104/pp.106.085274.

21. Froger, A. and Hall, J. E. (2007) 'Transformation of plasmid DNA into E. coli using the heat shock method', Journal of Visualized Experiments, (6). doi: 10.3791/253.

22. Fu, D.-Q. et al. (2006) 'Enhancement of virus-induced gene silencing in tomato by low temperature and low humidity.', Molecules and cells, 21(1), pp. 153-160. Available at: http://www.ncbi.nlm.nih.gov/pubmed/16511359.

23. Hamilton, A. J. and Baulcombe, D. C. (1999) 'A Species of small antisense RNA in posttranscriptional gene silencing in plants', Science, 286(5441), pp. 950-952. doi: $10.1086 / 327380$.

24. Heinlein, M. (2015) Plasmodesmata, Methods and Protocols. Edited by M. Heinlein. Hatfield, UK: Humana Press. doi: 10.1007/978-1-4939-1523-1.

25. Kalantidis, K. et al. (2008) 'RNA silencing movement in plants.', Biology of the Cell. 100(1), pp. 13-26. doi: 10.1042/BC20070079.

26. Kundu, L. M. and Ray, S. (2017) 'Mitotic abnormalities and micronuclei inducing potentials of colchicine and leaf aqueous extracts of Clerodendrum viscosum Vent. in Allium cepa root apical meristem cells', Caryologia, 70(1), pp. 7-14. doi: 10.1080/00087114.2016.1254452.

27. Lambing, C., Franklin, F. C. H. and Wang, C.-J. R. (2017) 'Understanding and manipulating meiotic recombination in plants.', Plant Physiology, 173(3), pp. 1530-1542. doi: 10.1104/pp.16.01530.

28. Liu, B., De Storme, N. and Geelen, D. (2018) 'Cold-induced male meiotic restitution in Arabidopsis thaliana is not mediated by GA-DELLA signaling', Frontiers in Plant Science, 9(2), pp. 1-11. doi: 10.3389/fpls.2018.00091.

29. Liu, E. and Page, J. E. (2008) 'Optimized cDNA libraries for virus-induced gene silencing (VIGS) using Tobacco rattle virus', Plant Methods, 4(5). doi: 10.1186/1746-4811-4-5.

30. Liu, Y. et al. (2002) 'Tobacco Rar1, EDS1 and NPR1/NIM1 like genes are required for Nmediated resistance to Tobacco mosaic virus', Plant Journal, 30(4), pp. 415-429. doi: 10.1046/j.1365-313X.2002.01297.x.

31. Nimchuk, Z. et al. (2000) 'Eukaryotic fatty acylation drives plasma membrane targeting and enhances function of several type III effector proteins from Pseudomonas syringae', Cell, 101(4), pp. 353-363. doi: 10.1016/S0092-8674(00)80846-6.

32. Padmanabhan, M. and Dinesh-Kumar, S. P. (2009) 'Virus-induced gene silencing as a tool for delivery of dsRNA into plants', Cold Spring Harbor Protocols, 4(2). doi: 10.1101/pdb.prot5139.

33. Peterson, R., Slovin, J. P. and Chen, C. (2010) 'A simplified method for differential staining of aborted and non-aborted pollen grains', International Journal of Plant Biology, 1(2), pp. 66-69. doi: 10.4081/pb.2010.e13. 

induced gene silencing (VIGS) in Arabidopsis thaliana

34. Pflieger, S. et al. (2008) 'Efficient virus-induced gene silencing in Arabidopsis using a "onestep" TYMV-derived vector', Plant Journal, 56(4), pp. 678-690. doi: 10.1111/j.1365313X.2008.03620.x.

35. Preuss, D., Rhee, S. Y. and Davis, R. W. (1994) 'Tetrad analysis possible in Arabidopsis with mutation of the QUARTET (QRT) genes.', Science, 264(5164), pp. 1458-1460. doi: 10.1126/science.8197459.

36. Pyott, D. E. and Molnar, A. (2015) 'Going mobile: Non-cell-autonomous small RNAs shape the genetic landscape of plants', Plant Biotechnology Journal, 13(3), pp. 306-318. doi: 10.1111/pbi.12353.

37. Ratcliff, F., Martin-Hernandez, A. M. and Baulcombe, D. C. (2001) 'Tobacco rattle virus as a vector for analysis of gene function by silencing', Plant Journal, 25(2), pp. 237-245. doi: 10.1046/j.0960-7412.2000.00942.x.

38. Ravi, M. and Chan, S. W. L. (2010) 'Haploid plants produced by centromere-mediated genome elimination', Nature. 464(7288), pp. 615-618. doi: 10.1038/nature08842.

39. Rhee, S. Y. and Somerville, C. R. (1998) 'Tetrad pollen formation in quartet mutants of Arabidopsis thaliana is associated with persistence of pectic polysaccharides of the pollen mother cell wall', Plant Journal, 15(1), pp. 79-88. doi: 10.1046/j.1365-313X.1998.00183.x.

40. Rodriguez, E. M. et al. (2001) 'Colchicine promotes a change in chromosome structure without loss of sister chromatid cohesion in prometaphase I-arrested bivalents.', Chromosoma., 110(7), pp. 478-486.

41. Ross, K. J., Fransz, P. and Jones, G. H. (1996) 'A light microscopic atlas of meiosis in Arabidopsis thaliana.', Chromosome Research, 4(7), pp. 507-516. doi: 10.1007/BF02261778.

42. Ruiz, M. T., Voinnet, O. and Baulcombe, D. C. (1998) 'Initiation and maintenance of virusinduced gene silencing.', The Plant Cell, 10(6), pp. 937-946.

43. Séguéla-Arnaud, M. et al. (2017) 'RMI1 and TOP3a limit meiotic CO formation through their C-terminal domains', Nucleic Acids Research, 45(4), pp. 1860-1871. doi: 10.1093/nar/gkw1210.

44. Senthil-Kumar, M., Lee, H.-K. and Mysore, K. S. (2013) 'VIGS-mediated forward genetics screening for identification of genes involved in nonhost resistance', Journal of Visualized Experiments, 78, pp. 1-10. doi: 10.3791/51033.

45. Senthil-Kumar, M. and Mysore, K. S. (2011) 'New dimensions for VIGS in plant functional genomics', Trends in Plant Science. Elsevier Ltd, 16(12), pp. 656-665. doi: 10.1016/j.tplants.2011.08.006.

46. Senthil-Kumar, M. and Mysore, K. S. (2014) 'Tobacco rattle virus-based virus-induced gene silencing in Nicotiana benthamiana', Nature Protocols, 9(7), pp. 1549-1562. doi: 10.1038/nprot.2014.092.

47. Serra, H. et al. (2018) 'Massive crossover elevation via combination of HEl10 and recq4a recq $4 \mathrm{~b}$ during Arabidopsis meiosis.', Proceedings of the National Academy of Sciences, 115(10), pp. 2437-2442. doi: 10.1073/pnas.1713071115.

48. De Storme, N., Copenhaver, G. P. and Geelen, D. (2012) 'Production of diploid male gametes in Arabidopsis by cold-induced destabilization of postmeiotic radial microtubule arrays', Plant Physiology, 160(4), pp. 1808-1826. doi: 10.1104/pp.112.208611.

49. Susi, P. et al. (2004) 'Characteristics of RNA silencing in plants: similarities and diferences across kingdoms', Plant Molecular Biology, 54(2), pp. 157-174.

50. Wang, J. et al. (2017) 'High temperature-induced production of unreduced pollen and its cytological effects in Populus', Scientific Reports. Springer US, 7(5281). doi: 

induced gene silencing (VIGS) in Arabidopsis thaliana

10.1038/s41598-017-05661-x.

51. Wijnen, C. L. et al. (2018) 'A complete chromosome substitution mapping panel reveals genome-wide epistasis in Arabidopsis', bioRxiv. Available at: http://biorxiv.org/content/early/2018/10/05/436154.abstract.

52. Wijnker, E. et al. (2012) 'Reverse breeding in Arabidopsis thaliana generates homozygous parental lines from a heterozygous plant', Nature Genetics, 44(4), pp. 467-470. doi: 10.1038/ng.2203.

53. Wijnker, E. et al. (2013) 'The genomic landscape of meiotic crossovers and gene conversions in Arabidopsis thaliana', eLife, 17(2), pp. 1-22. doi: 10.7554/eLife.01426.

54. Wijnker, E. and de Jong, H. (2008) 'Managing meiotic recombination in plant breeding', Trends in Plant Science, 13(12), pp. 640-646. doi: 10.1016/j.tplants.2008.09.004.

55. Wu, C., Lingling, J. and Goggin, F. (2011) 'The reliability of virus-induced gene silencing experiments using Tobacco rattle virus in tomato is influenced by the size of the vector control', Molecular Plant Pathology, 12(3), pp. 299-305. doi: 10.1111/j.13643703.2010.00669.x.

56. Xie, Z. et al. (2004) 'Genetic and functional diversification of small RNA pathways in plants', PLoS Biology, 2(5), pp. 642-652. doi: 10.1371/journal.pbio.0020104.

57. Younis, A., Hwang, Y.-J. and Lim, K.-B. (2014) 'Exploitation of induced 2n-gametes for plant breeding', Plant Cell Reports, 33(2), pp. 215-223. doi: 10.1007/s00299-013-1534-y. 
To the marvelous and paradigmatic nature of viruses.

"An inefficient virus kills its host.

A clever virus stays with it."

James Lovelock 


\section{Chapter 6 \\ General discussion.}




\section{An introduction to the Importance and potential for modifying plant meiosis.}

The past years have seen a clear increase in the interest to modify meiosis to accelerate plant breeding (Wijnker and de Jong, 2008; Lambing, Franklin and Wang, 2017). This in part resulted from the discovery of various meiotic mutant phenotypes that potentially had significant breeding applications, such as increasing recombination frequencies (Girard et al., 2015; Séguéla-Arnaud et al., 2015, 2017; Fernandes et al., 2017) or the induction of $2 \mathrm{n}$ gamete formation (Chen, 2010; Sattler, Carvalho and Clarindo, 2016). In addition to the use of such mutants, several papers described possible breeding applications including reverse breeding or synthetic apomeiosis (D'Erfurth et al., 2009; Dirks et al., 2009; Wijnker et al., 2012; Mieulet et al., 2016). Furthermore, it was shown that it was possible to phenocopy mutant phenotypes by using chemicals or abiotic stress (Kato, 2002; De Storme, Copenhaver and Geelen, 2012; Lambing, Franklin and Wang, 2017). Yet, the available methods to induce newly discovered meiotic phenotypes rely mainly on stable transformants or mutants. As many of the economically relevant crops have a long generation time, mutants are not readily available. In addition, crop species are often recalcitrant to transformation, whereas translation of meiotic mutant phenotypes from one species to the other is not straightforward. It also may not always be beneficial to introduce mutant alleles in breeding populations, as it can lead to partial or complete sterility (Couteau et al., 1999; Dion et al., 2007; Higgins et al., 2008; Da Ines et al., 2013; Zhang et al., 2017).

A short overview of the work described in my thesis.

In this thesis I have explored the potential of virus-induced gene silencing (VIGS) to induce a wide range of meiotic phenotypes in Arabidopsis thaliana. Chapter 2 presented the versatility of VIGS for altering four different cell processes: sister-chromatid cohesion, crossover formation, the second meiotic division, and haploid spore separation after meiosis. In subsequent chapters more detailed questions were addressed, asking whether VIGS can help to improve the efficiency of reverse breeding (Dirks et al., 2009; Wijnker et al., 2012). I showed that, by targeting class I crossovers (hereafter COs) in meiosis, it is possible to develop new reverse breeding strategies based on partial crossover suppression (Chapter 3). I also explored the possibility to use VIGS to increase recombination frequencies (Chapter 4), and to promote the further use of the technique, I detailed the precise method for targeting meiotic genes in Arabidopsis in a comprehensive protocol (Chapter 5). Based on these experiments, I can conclude that VIGS is a versatile method to modify meiosis in Arabidopsis. Furthermore, the availability of several VIGS systems holds a strong promise for the application of VIGS-strategies for meiosis in numerous plants species, for instance, cereal crops including maize, barley, rice, oat, and wheat (Holzberg et al., 2002; Pacak et al., 2010; Bennypaul et al., 2012; Wang et al., 2016), vegetable crops such as Solanum species, potato, soybean, common bean (Liu, Schiff and Dinesh-Kumar, 2002; Faivre-Rampant et al., 2004; Díaz-Camino et al., 2011; Juvale et al., 2012). Viral vectors 
are also available for other economically important plants such as cotton, cassava, and rosids (Fofana et al., 2004; Becker, 2013; Pang et al., 2013). All these species represent putative candidates to use VIGS for meiosis, but the translation from Arabidopsis to other species certainly requires additional research.

What needs to be addressed regarding the application of VIGS-for-meiosis.

The main goal of my thesis was to explore whether VIGS could be used to target meiosis with the underlying thought of later applying these methods to other species. My experiments in Arabidopsis provide a good insight into the possible applications but also the limitations of using VIGS to modify genetic control of meiotic processes. To some extent, these limitations may be inherent to a VIGS system in Arabidopsis, and interestingly, some of the applications that are not possible in this model species may be more easily feasible in others. In the following I will first present a summary of strengths and weaknesses of using VIGS for meiosis. I will use this to discuss how these challenges can be overcome, not limiting myself to Arabidopsis but considering other species and VIGS-systems as well. With the knowledge drawn from this thesis, it is possible to describe the ideal setup of how VIGS-for-meiosis would be in other species. The most direct applications of VIGS-for-meiosis lies in the knockdown of single genes, and resulting applications, as shown in my thesis for the induction of $2 \mathrm{n}$ gamete formation (Chapter 2) and the knockdown of crossover formation to develop new reverse breeding strategies (Chapter 3). For this reason, I will explain the application of polyploidization and reverse breeding in crops in higher detail in the second part of my discussion.

\section{How to translate the use of VIGS to efficiently modify meiosis in plants}

SWOT analysis: VIGS for meiosis in plants.

By setting up a protocol to induce meiotic phenotypes using VIGS in Arabidopsis, and by testing the induction of a multitude of phenotypes using VIGS, it is possible to present an overview of the strengths and weaknesses of "VIGS-for-meiosis". From this, it is possible to look forward to what the technique may be used for, but also what may prohibit the application of VIGS for various reasons. These points are summarized in an analysis of the strengths, weaknesses, opportunities and threats of using VIGS-formeiosis (SWOT; Figure 1).

The strongest points of VIGS-for-meiosis lie certainly in its transient nature and in its speed of experimental use, which enable one to target many genes, as was successfully done for DMC1, SDS, PRD1, REC8, OSD1, MSH5, QRT1, QRT2, RECQ4/FIGL1 genes (Chapters 2 to 4). VIGS achieves a dominant knockdown of a specific gene without associated transgenes or mutations segregating among offspring. Arguably, the best example was described in Chapter 3, where VIGS was used for reverse breeding. Several weaknesses that have previously been described for the application of VIGS do also apply to VIGS-for-meiosis including, genotype-dependency 
for the susceptibility to VIGS constructs, its transient nature, and incomplete penetrance. The latter two have as a consequence that in Arabidopsis the phenotype will be present for only a (short) period of time and not all the inoculated plants will show a silencing phenotype (Chapters 2 to 4). Moreover, there are also important limitations that are more specific to VIGS in meiosis. An example of such limitations in the current toolbox is the apparent inability of knocking down two genes simultaneously. This is an important limitation to induce meiotic phenotypes that do not exhibit strong pollen phenotypes and hence require a phenotypic marker to identify silenced gametes. Opportunities for the use of VIGS in meiosis arise mainly in its possible applications in crops, especially to obtain meiotic phenotypes and to conduct reverse genetic analysis in species that are recalcitrant to stable transformation. The possibility of successfully establishing VIGSfor-meiosis in large number of species could significantly increase the efficiency of developing breeding techniques using meiotic phenotypes, especially in the USA, where VIGS-derived lines would be allowed for commercial breeding. The main threats to the application of the method in a breeding set-up relate to the regulations applied in the European Union as the method is considered GMO, and therefore not suitable for commercial breeding. Furthermore, variable growing conditions greatly affect the penetrance of the silenced phenotype which may pose a significant limitation when it comes to the application of VIGS in the field. This may also be complicated when there is risk of cross-contamination to other plants.

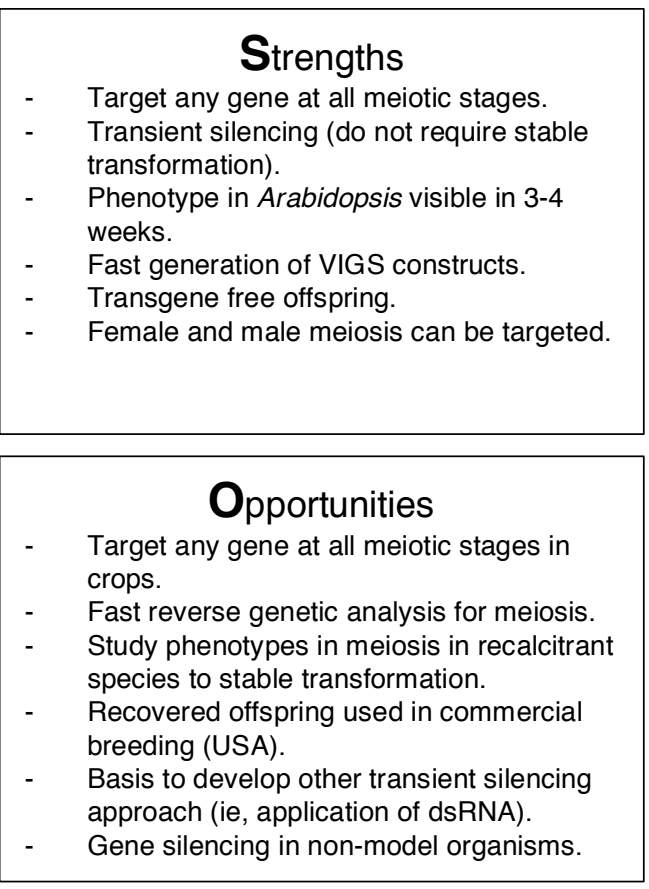

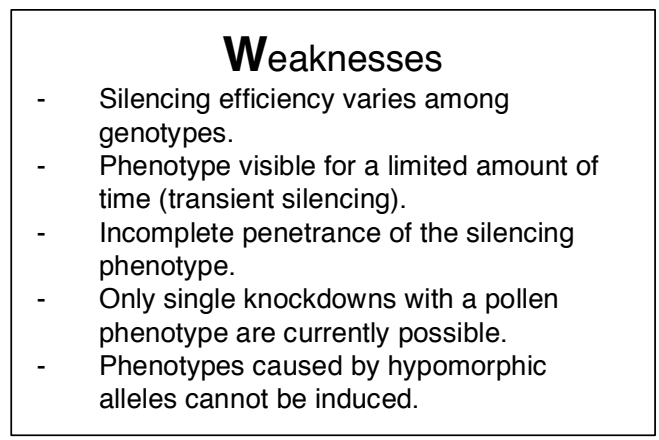

\section{Threats}

- $\quad$ Efficient VIGS systems may not available for the species of choice.

- Characterization of gene function may be easier using mutants or knockouts.

- $\quad$ GMO in EU and some other parts in the world.

- Viral presence may persist over generations in some species (Not suitable for commercial breeding).

- $\quad$ Application in crops grown in the field.

Figure 1. SWOT analysis of VIGS-for-meiosis in Arabidopsis and crops. 
The SWOT analysis presented in Figure 1 identifies three main issues that deserve special attention. Firstly, it is important to use a phenotypic marker as a robust positive control for validating VIGS silencing in reproductive tissues. Secondly, it is important that the silencing activity remains present from the moment of inoculation to the time of flower development, in order to make sure that efficient silencing will happen in the meiocytes. Thirdly, simultaneous silencing of two or more endogenous genes in meiosis is key to take advantage of the full potential of VIGS. For instance, this will allow the induction of the double knockdown of a marker such as QRT genes, to obtain gametes that are silenced for the meiotic target and display a qrt phenotype. In this way, one could visually confirm a knockdown and at the same time discriminate silenced gametes for both targets by selecting pollen tetrads. Hereafter I will address these three points in more detail.

\subsection{Establishment of robust phenotypic markers.}

Silencing of genes to be used as a macroscopic phenotypic marker for positive VIGS.

The knock-down of targets to be used as phenotypic markers not only serve to verify the silencing without further compromising plant health; rather they are also very informative and convenient to directly select flower buds or flowers that are reliably affected by the silencing, reducing labor-intensive phenotyping of inoculated plants. In this thesis I used the silencing of PDS as positive control to monitor silencing throughout plant development. Silencing of PDS causes photobleaching symptoms that are easy to identify and allow for a rough assessment of silencing progression over time (Becker and Lange, 2009). However, PDS silencing compromises plant health, and results in retarded plant growth which also may affect gamete viability. Therefore, $P D S$ cannot be used as a marker for VIGS in meiosis. It would be possible to target other genes instead that are expressed in, or near reproductive tissues, that do not compromise plant health but do allow monitoring the activity of VIGS silencing in reproductive tissues. In Chapter 2 I showed the possibility of observing silencing in flowers by inducing the formation of pollen tetrads using VIGS to target $Q R T 1$ and $Q R T 2$. The downside of the use of quartet phenotypes is that one needs a microscope to assess the progression of VIGS silencing in reproductive tissues. It would therefore be interesting to also pursue other phenotypic markers. For instance, a change in petal or sepal number, color, or morphology could clearly and visually identify the flower affected by the silencing. One example is the PERIANTHIA gene (PAN) in Arabidopsis thaliana that control the number of petals. Arabidopsis mutants in PAN usually display five petals instead of the wildtype four (Chuang et al., 1999). Another example would be targeting a flower-specific b-ring carotene hydroxylase gene, CrTR-b2 in tomato, that leads to a change in petal color from yellow to white (Galpaz et al., 2006). More examples of numerous flower pigments in several plant species can be found in the literature (Tanaka, Sasaki and Ohmiya, 2008). 


\section{GFP marker coupled to viral signal.}

An intracellular phenotypic marker that does not involve the knockdown of an endogenous gene could also be used to assess the spread pattern of the virus in the host. Actual viral movement in the plant could be evaluated using a modified VIGS vector in which a GFP-tag has been fused to the coat protein of the virus (Tian et al., 2014). This will deliver a visual and direct confirmation of the ability of the virus to potentially penetrate the meiocytes by scanning developing flower buds with a UV lamp to track the presence of GFP signal. However, the viral signal can be amplified, as viral siRNAs can be transported and reach other cells that are far from the replication spots of the virus (Susi et al., 2004; Ueki and Citovsky, 2005; Kalantidis et al., 2008; Becker and Lange, 2009; Pyott and Molnar, 2015). This means that silencing can also take place in the absence of the virus in a tissue, and therefore the direct monitoring of viral spread does not necessary correspond to real induced silencing. VIGS can very efficiently silence the expression of GFP in a transgenic plant (Peele et al., 2001; Burch-Smith et al., 2006; Quadrana et al., 2011; Pflieger et al., 2014), and this is likely the most efficient way to thoroughly assess real-time silencing in the host: the phenotype can be monitored faithfully from the moment of inoculation simply by validating the absence of GFP signal in the tissue.

\subsection{VIGS system and inoculation method}

The chances of inducing meiotic phenotypes depend on the virus or the viral siRNAs reaching the meiocytes (Kalantidis et al., 2008; Becker and Lange, 2009). The relative short life-span of RNA virus such as TRV of only a few weeks (Ruiz, Voinnet and Baulcombe, 1998; Burch-Smith et al., 2004; Bernacki et al., 2010), is a real limitation to target meiocytes. This is especially true if flowering occurs after several months of growth (e.g. such as in maize and barley) because the viral infection will be long overcome by the time the plant flowers. However, agroinoculating plants at a later moment may result in poor silencing efficiency (Burch-Smith et al., 2006; Padmanabhan and Dinesh-Kumar, 2009; Agüero et al., 2014). Agroinoculation via leaf-infiltration in older tissue is practically challenging, because a lower amount of total bacteria inoculum can be delivered to the plant. Furthermore, plasmodesmata apertures and complexity of the leaf architecture are likely to interfere with the potential of the virus or the viral signal to spread from cell to cell, resulting in a lower accumulation of the virus in the tissue (Burch-Smith et al., 2004; Kalantidis et al., 2008; Heinlein, 2015; Pyott and Molnar, 2015). For this reason, for a successful application of TRV for meiosis in Arabidopsis, the moment of inoculation had to be precisely timed such that it was early enough to promote efficient virus accumulation in the plant, but late enough to make sure that silencing reached its highest peak when the meiocytes are present (Chapter 5). For Arabidopsis and other species, there are other ways to ensure efficient silencing in meiosis. In the following section I will explain that there are VIGS strategies using viruses 
that are stable for longer time. Thereafter, I will address techniques that allow for inoculation during later life stages.

VIGS systems based on DNA virus may be more stable over time.

Differences in the time that the virus remains stable in the host have been found among the different viral systems used for VIGS, especially when comparing RNA viruses, such as TRV or BSMV (Barley stripe mosaic virus) to DNA viruses (i.e. Geminiviruses) (Turnage et al., 2002; Burch-Smith et al., 2004; Bernacki et al., 2010; Senthil-Kumar and Mysore, 2011). RNA viruses are sometimes active for only a few weeks (Holzberg et al., 2002; Burch-Smith et al., 2004), whereas silencing induced by some geminiviruses can persist throughout the whole life of the plant, even when the plants are inoculated as young seedlings, as was shown for cotton and wheat (Tuttle et al., 2008; Bernacki et al., 2010; Senthil-Kumar and Mysore, 2011; Bennypaul et al., 2012). Therefore, transition towards a DNA-virus based VIGS system to target genes in meiosis will likely boost the penetrance of silencing and, in addition, they will be more suitable to target meiosis in a species with long-generation times: the longer a virus remains active, the higher the chance that meiosis will be targeted.

Alternatives to Agrobacterium-mediated inoculation can trigger silencing in older tissues.

Alternatives to Agrobacterium-mediated inoculation may be more attractive to target meiosis. The majority of current protocols use agroinoculation as the favorite method to deliver viral vectors (Vaghchhipawala et al., 2011). However, agroinoculation via leafinfiltration is mainly efficient when seedlings are treated (Burch-Smith et al., 2006; Vaghchhipawala et al., 2011; Bilichak and Kovalchuk, 2017). Thus, the use of different methods of VIGS inoculation may allow successful virus delivery and infection shortly before the plant starts flowering. These strategies have all in common that they are suitable for species that are recalcitrant to Agrobacterium transformation. For example, (Bennypaul et al., 2012) illustrated that viral siRNAS derived from the RNA-tripartite viral system BSMV could induce successful silencing of DMC1 in wheat. The use of BSMV should certainly be revisited to test whether it is possible to breed with gametes resulting from VIGS-modified meiosis. A particular promising approach is the use of crude extracts containing viral particles of a plant infected with a VIGS vector to inoculate a second target plant. For instance, Wang et al., 2016 used a vascular puncture method to inoculate maize kernels with the crude extract from VIGS treated Nicotiana benthamiana plants (Wang et al., 2016). Strikingly, at least $80 \%$ of the inoculated plants displayed silencing symptoms in seven out of the 18 treated inbred lines. A third possible method is the bombardment of plant tissues with the components of a DNA virus, which would certainly allow that plants are treated in later developmental stages (Peele et al., 2001; Turnage et al., 2002; Fofana et al., 2004; Tao and Zhou, 2004; Bernacki et al., 2010). In addition to methods such as bombardment, it has also been described that consecutive booster inoculations with viral particles can sustain the silencing phenotype 
over time, and this may ensure continued silencing up to meiosis (Senthil-Kumar and Mysore, 2014). Lastly, an interesting approach was developed for Arabidopsis and common bean (Phaseolus vulgaris $L$ ) by the use of a "one-step" VIGS vector based on the Turnip yellow mosaic virus (TYMV) and the Bean pod mottle virus (BPMV), respectively (Pflieger et al., 2008, 2014). Leaf abrasion with the intact DNA plasmid harboring a short cDNA host sequence cloned in antisense induces silencing of the target gene in meristematic tissues and flowers (Pflieger et al., 2008). Thus, it is very possible that using DNA viral vectors for VIGS and/or alternatives to Agrobacteriummediated inoculation may provide better approaches to target meiosis in any species, regardless the time between inoculation and bolting. In addition, higher penetrance of the silencing phenotype, conditioned by a higher stability of the virus, may be expected.

1.3 Pushing the potential of VIGS forward: Simultaneous silencing of endogenous genes in meiosis.

As only part of the meiotic mutant phenotypes necessarily displays an aberrant pollen phenotype, silencing of some meiotic targets is not expected to induce an obvious phenotype in pollen. Therefore, having the possibility of knocking down the expression two genes (e.g. QRT and a meiotic gene) simultaneously is one of the most desired tools to further develop VIGS-for-meiosis. In Chapter 2 and 4, I aimed at silencing simultaneously PRD1-REC8-OSD1 and FIGL1-RECQ4-QRT2, to induce more complex meiotic phenotypes in Arabidopsis. These constructs were to induce mitotic-like division - mimicking synthetic apomeiosis; MiMe (D’Erfurth et al., 2009) or to achieve a strong increase in recombination frequencies (Fernandes et al., 2017). However, it was not possible to confirm the simultaneous knockdown of genes in these experiments. In the following paragraphs I will discuss how changes in insert size, insert orientation, and type of viral vector used (DNA-based vs RNA-based) may help to enable a double knockdown for a large number of species.

Insert characteristics.

Insert stability within the viral vector ultimately determines the potential to induce silencing (Senthil-Kumar and Mysore, 2011; Wu, Lingling and Goggin, 2011; Becker, 2013; Ding et al., 2018). Although the mechanisms that precisely cause a reduction of stability of the vector have not been fully elucidated, it seems that longer inserts are more readily removed from the virus through recombination (Bruun-Rasmussen et al., 2007; Liu and Page, 2008; Becker, 2013). Therefore, it is expected that several target sequences fused in the same insert may reduce insert stability in the vector. To overcome this problem, modification of the insert characteristics and/or the viral system can be explored (Peele et al., 2001; Ding et al., 2018). Cloning of shorter target sequences or short inverted repeats can increase silencing of the target gene and at the same time reduce total insert length to promote insert stability (Peele et al., 2001; Lacomme, Hrubikova and Hein, 2003; Pflieger et al., 2008; Senthil-Kumar and Mysore, 
2011; Wu, Lingling and Goggin, 2011; Ding et al., 2018). For instance, Peele et al., 2001 and Wang et al., 2016 showed successful silencing of endogenous genes using target sequences of less than 100bp and 100bp-300bp, respectively. In Chapter 2, I have also shown that a fragment of 116 bp could silence DMC1 in Arabidopsis. Lacomme et al., 2003 showed that silencing of PDS in Nicotiana benthamiana using TMV carrying shortrepeated sequences of 40-60 bp induced a more severe phenotype than CDNA sequences cloned in both sense and antisense. In conclusion, short sequences cloned as inverted repeats will likely promote that the target gene is silenced for a longer time and at the same time, increase the penetrance of the silencing phenotype in meiosis (Peele et al., 2001; Lacomme, Hrubikova and Hein, 2003).

\section{DNA-based viral vectors may be preferred for simultaneous silencing}

In order to achieve a simultaneous knockdown of two targets, a simple strategy consists in inoculating the plant with two different viral vectors, one vector for each target gene. However, in some cases this leads to a lower penetrance of the silencing phenotype (Pang et al., 2013). Possibly, lower silencing efficiency resulted from infections with two distinct viral vectors may have negatively altered viral population dynamics reciprocally (Susi et al., 2015). Consequently, the better strategy likely lies in investing on the optimization to use a single VIGS vector rather than co-silencing multiple targets with two different vectors (Burch-Smith et al., 2006; Pang et al., 2013; Senthil-Kumar and Mysore, 2014). Interestingly, DNA-based vectors seem better able to silence two or more endogenous genes, which is probably related to the higher stability of this virus previously discussed (Bernacki et al., 2010). This was for instance shown for Nicotiana benthamiana, using TGMV: a DNA bipartatite geminivirus-derived VIGS vector (Peele et al., 2001). The novelty in this case was that the two components of the virus-derived vector were engineered to allow cloning of foreign inserts. Also in Arabidopsis a double knockdown was achieved through direct bombardment with a geminivirus-based vector CbLCV (Turnage et al., 2002) in which both viral components of the bipartite DNA VIGS system (i.e. CbLCVA and CbLCV B) (Turnage et al., 2002) allowed insertion of target sequences. Tao et al., 2004 described simultaneous silencing in tomato using a DNA modified virus begomovirus/DNAbeta system to target endogenous genes and transgenes (Tao and Zhou, 2004). To our knowledge, TRV has only been successful to silence transgenic GFP and PDS simultaneously in Arabidopsis and tomato (BurchSmith et al., 2006; Quadrana et al., 2011), and only Pang et al., 2013 showed silencing of endogenous genes in vegetative tissues of Gossypium barbadense (cotton) using a single TRV vector (Pang et al., 2013). Yet, the modification of TRV described by Deng et al., 2013 allows systemic silencing of target genes by cloning inserts in both components of TRV-based VIGS system - TRV1 and TRV2 (Deng et al., 2013). This could also be explored to further develop the technique in the wide range of TRV host species, including Arabidopsis. 


\section{Applications for crop breeding based on meiosis modifications.}

In this thesis I have shown that transient silencing of genes in meiosis using VIGS is a reliable and rapid method to change genetic inheritance (Chapter 2; production of tetraploid offspring using SDR gametes) and to develop breeding applications (Chapter 3; developing efficient reverse breeding). It is interesting to explore what roles these applications could play in modern plant breeding practices. In the following sections I will first discuss how to exploit the use of VIGS for polyploid production. Secondly, I will describe how the translation of reverse breeding to crops can be addressed. While VIGS can certainly be a part of this, I will discuss also alternatives that may be more convenient to modify meiosis for reverse breeding approaches. Reverse breeding can be used for efficient hybrid breeding or for the generation of mapping populations. Depending on the species or aim of the breeding program, the choice of silencing technique may vary.

\subsection{To date VIGS is the most targeted, side-effect-free, approach to produce polyploids}

Production of polyploids is a valuable resource in plant breeding. Polyploid production can be used to overcome inter-specific barriers (Tonosaki et al., 2018) or to enhance plant performance (i.e. larger floral organs, fruits, yield) (Sattler, Carvalho and Clarindo, 2016). Polyploid production in plant breeding is often achieved through the generation of diploid gametes (2n). Although production of $2 n$ gametes may be formed spontaneously in several crop varieties, the rate can vary greatly (Dewitte et al., 2009; Younis, Hwang and Lim, 2014). The application of heat or cold shock (Mason et al., 2011; De Storme, Copenhaver and Geelen, 2012; Wang et al., 2017), as well as the use of chemicals such as nitrous oxide, oryzaline, or colchicine have been used to obtain $2 n$ gametes. However, generally these treatments have downsides: the exact mode of action in most cases remains unknown, plus they may cause additional undesired effects in meiosis and mitosis (Rodriguez et al., 2001; Kato, 2002; Harsant et al., 2013; Younis, Hwang and Lim, 2014; Kundu and Ray, 2017). In addition, one cannot strictly control whether the gametes produced result from omission of the first or the second meiotic division, which may leave uncertainty on the precise genetic make-up of the gametes and offspring (Younis, Hwang and Lim, 2014).

Using VIGS one can generate polyploids and at the same time control genetic inheritance. This was shown in Chapter 2: transgene-free tetraploid offspring were recovered from TRV2 inoculated plants targeting the OSD1, which silencing induces a second meiotic restitution. This proved that VIGS can efficiently modify both male and female meiosis simultaneously. In addition, the offspring plants were true tetraploid, lacking the diploid-polyploid chimera's that may occur after colchicine treatment. Prusicki, thesis 2018, exploited the efficiency of the TRV-OSD1 VIGS construct used in Chapter 2 to generate Arabidopsis tetraploids of fluorescent reporter lines. The tetraploid progeny was consequently used to study meiotic progression using a life 
imaging technique (Prusicki et al., 2018). Thus, VIGS can be used in plant breeding to preferentially induce a desired type of unreduced gamete (FDR or SDR). This feature may be of great use for instance in lily breeding (Lim et al., 2004; Van Tuyl et al., 2005). In lily, occurrence of diploid gametes has been described for various interspecific hybrids. Yet, whether either the unreduced gametes produced are FDR or SDR was dependent on the hybrid background (Lim et al., 2001, 2004). The controlled production of SDR may be interesting to enhance genetic variation and to introgress desired allele combinations in hybrid polyploid backgrounds (Lim et al., 2004). On the other hand, in FDR gametes, which result from suppressing the first meiotic division, the segregation of alleles will likely be lower. To induce FDR, one could target male meiosis through orthologs of the Arabidopsis genes PARALLEL SPINDLE 1 (PS1) or JASON (D'Erfurth et al., 2008; De Storme and Geelen, 2011).

2.2 Downregulation of recombination frequencies is the key to producing new hybrids and mapping populations in high chromosome number species.

In Chapters 2 and 3, I have shown that the transient regulation of meiotic genes is efficient for generating offspring derived from both female and male silenced gametes. This showed that meiotic inheritance can be manipulated at will, making it potentially applicable for plant breeders. Chapter 3 describes one of the most elaborated examples to date of how the targeted modification of meiosis can lead to a breeding application in a plant. It was argued that partial, rather than complete suppression of crossover recombination may be the most efficient approach for developing reverse breeding-like techniques in crops. The downregulation of crossover recombination also leads to a visual pollen phenotype that is easy to verify through high rates of pollen abortion. Because haploid and $\mathrm{DH}$ technologies have been described for some of the most economically relevant crops (see Table 2), many elements are in place to pursue reverse breeding in an actual breeding set-up. In the next example I will discuss how reverse breeding through partial crossover suppression may become feasible in a crop. Reverse breeding not only generates parental lines for heterozygous plants, but is also a method for the fast generation of chromosome substitution lines in Arabidopsis and in crops.

As an example, I will discuss the feasibility of applying reverse breeding technology in maize. I already described that stable and efficient VIGS strategies can be applied in this crop, so the knockdown of meiotic genes appears feasible (Wang et al., 2016; Mei and Whitham, 2018). Even though class I crossover mutants have not been described for maize, it is more than likely that reverse breeding can be achieved through partial crossover suppression. Falque et al., 2009 estimated that in maize, similar to Arabidopsis thaliana an interfering-dependent and independent pathways regulate crossover formation, and that the non-interfering pathway accounts (on average) for about $15 \%$ of the total number of COs (Higgins et al., 2008; Falque et al., 2009). In addition, recent analysis have identified the homologous sequences to the Arabidopsis 
MUS81 and MSH4 in the maize genome (Sidhu, Warzecha and Pawlowski, 2017). Therefore, it would be very useful to investigate as to whether class I COs mutant phenotypes can be induced using VIGS.

The average number of COs in maize $(x=10)$ varies considerably between cells, but also between varieties, with estimated average numbers of 20.5 - 38 COs per meiosis (Anderson et al., 2003; Li, Li and Yan, 2015). An 85\% decrease of COs rates would imply that $\mathrm{CO}$ numbers could be reduced to about three - six COs per meiosis. The difference in residual crossover incidence of three versus six also affects the chances of obtaining viable gametes that are expected $(1 / 2)^{(10-3)}=(1 / 2)^{7}=0,00781$ and $(1 / 2)^{(10-6)}=$ $(1 / 2)^{4}=0,0625$ (considering only one COs per chromosome pair). These numbers are summarized in Table 1. Using the equations given in Chapter 3, the respective chances of finding $\mathrm{DH}_{0} / \mathrm{DH}_{1}$ range between $20 \%$ to $35 \%$ when the residual number of COs is three, and $3 \%$ to $12 \%$ when the residual number of COs is six. The residual crossover number results in an almost seven-fold increase in probability for obtaining $\mathrm{DH}_{0}(20 \%$ versus $3 \%$ ). Frequencies in obtaining $\mathrm{DH}_{1}$ differ about three-fold (35\% versus $12 \%$ ). If all $\mathrm{DH}_{0}$ or $\mathrm{DH}_{1}$ are considered to be useful offspring, the chance that random viable gametes give rise to useful $\mathrm{DH}_{0}$ and $\mathrm{DH}_{1}$ lines for reverse equals $55 \%$ and $15 \%$ for plants with three or six residual COs respectively.

Table 1: Chances of recovering $\mathrm{DH}_{0}$ and $\mathrm{DH}_{1}$ in a maize plant $(x=10)$. The chances of $\mathrm{DH}$ recovery depend on the reduction of $\mathrm{COs}$ and hence, residual $\mathrm{n}^{\circ}$ of $\mathrm{CO}$ in meiosis. In maize, $85 \%$ reduction of COs can leave in average 3 to 6 residual COs (Anderson et al., 2003; Li, Li and Yan, 2015). Based on the chances of finding non-recombinant and low recombinant gametes and the haploid and DH regeneration rate in maize it will lead to a similar number of final $\mathrm{DH}_{0}$ recovered. However, the number of $\mathrm{DH}_{1}$ recovered from meiosis with six residual COs is much higher. Note nevertheless, that $55 \%$ of the total number of viable gametes produced in a meiosis with three residual COs will be $\mathrm{DH}_{0}$ and $\mathrm{DH}_{1}$ offspring. This number on the other hand decreases (15\%) when only 3 residual C0s remain.

\begin{tabular}{|c|c|c|c|c|c|c|c|c|c|c|c|}
\hline 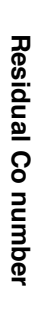 & 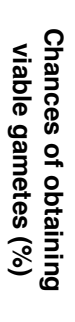 & 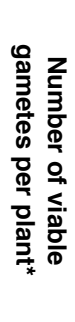 & 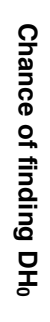 & 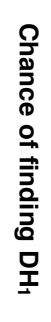 & 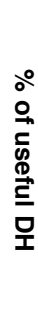 & 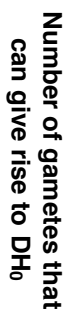 & 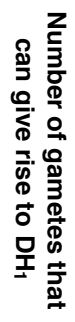 & 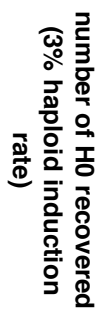 & 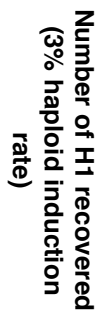 & 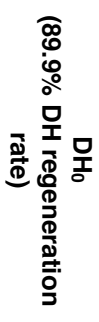 & 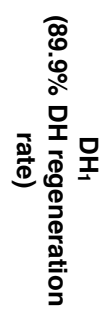 \\
\hline$\omega$ & $\begin{array}{l}\stackrel{0}{i} \\
\stackrel{\infty}{\longrightarrow} \\
\stackrel{0}{0}\end{array}$ & $\begin{array}{l}\vec{ज} \\
\text { N }\end{array}$ & $\begin{array}{l}\text { 응 } \\
\text { O }\end{array}$ & $\begin{array}{l}W \\
\text { No } \\
0 \\
0\end{array}$ & $\begin{array}{l}\text { ज़ } \\
\text { O }\end{array}$ & $\underset{\sim}{\vec{N}}$ & 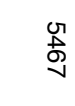 & $\stackrel{\leftrightarrow}{\not}$ & $\vec{\nexists}$ & $\stackrel{\infty}{\oplus}$ & $\vec{f}$ \\
\hline$\sigma$ & $\begin{array}{l}\text { D } \\
\text { N } \\
\text { o }\end{array}$ & $\begin{array}{l}\vec{N} \\
\text { Oे } \\
8\end{array}$ & $\begin{array}{l}\omega \\
\text { ○ }\end{array}$ & त्. & जo & $\begin{array}{l}\text { Wै } \\
\text { गै }\end{array}$ & $\begin{array}{l}\overline{0} \\
8\end{array}$ & $\vec{\omega}$ & 获 & 호 & $\stackrel{\Delta}{\phi}$ \\
\hline
\end{tabular}


A single tassel produces on average about 2-18million pollen grains (Schwartz and Osterman, 1976; O'Keeffe, 2009). If a minimum average of 2 million pollen grains is considered, F1 hybrids with three and six COs per meiosis, thus produce 15620 and 125000 viable gametes, respectively. Of these, 3124 and 3750 could be potentially be recovered as $\mathrm{DH}_{0}$ and 5467 / 15132 as $\mathrm{DH}_{1}$. So, since quite a number of viable gametes will be produced by such plants, it is possible to produce haploid offspring. There is a haploid inducer line available for maize that can be used as female in crosses, having a haploid induction rate of about 3\% (Kermicle, 1969). This would generate about 94 / 113 $\mathrm{DH}_{0}$ lines and 164 / $454 \mathrm{DH}_{1}$ per hybrid for three / six COs per genome. DH production rates through spontaneous doubling in maize depends on the genotype, ranging from $8.9 \%$ to $89.8 \%$ (Wu et al., 2016). In the case of low DH regeneration efficiency, a nitrousoxide (NO) treatment to induce chromosome doubling could be considered (Kato, 2002). Thus, taking a maximum of $\mathrm{DH}$ regeneration efficiency $(89.8 \%)$ into account, a final number of $84 \mathrm{DH}_{0}$ and $147 \mathrm{DH}_{1}$ would be obtained in the presence of three residual COs, and this number equals $101 \mathrm{DH}_{0}$ and $404 \mathrm{DH}_{1}$ if six residual COs are allowed.

Thus, while reducing the number of COs to three or six at first sight seems a trivial difference, it is clear from the above analysis that it is not. The main difference between both approaches is that in the case of a reduction of $\mathrm{CO}$ formation to three per meiosis, $55 \%$ of offspring will be putative breeding lines, whereas in the case of a reduction to six COs per meiosis, only $15 \%$ is of use. The latter technique for that reason will be more expensive because $\mathrm{DH}$ offspring with more than one $\mathrm{CO}$ should be discarded. Dirks et al., 2009 showed that for a species with ten chromosomes, one needs on average a total of $266 \mathrm{DH}$ for expecting a perfectly complementing pair (Dirks et al., 2009). This means that reverse breeding could be achieved by efficiently silencing with a minimum of three maize plants. This number will decrease if the efficiency of reverse breeding is calculated based on a number of starting gametes larger than 2 million. The same calculations can be applied to the species listed in Table 2.

Table 2: Haploid and DH technology in 6 different plant species. The different species are indicated in the first column while in the second column is shown the haploid number for the species. The third column lists the technologies described for haploid induction (i.e., Haploid-inducer lines, in vitro androgenesis, in vitro gynogenesis, mutations) and the double-haploid (DH) efficiency. Efficient DH production occurs through spontaneous doubling or applying nitrous oxide (NO). The rates of spontaneous doubling for all the crop-species is reviewed in Ren et al., 2017. 


\begin{tabular}{|c|c|c|c|}
\hline Species & $\mathrm{n}=\mathrm{x}$ & Haploid and DH technology & Reference \\
\hline Arabidopsis & $x=5$ & $\begin{array}{l}\text { Haploid production } \\
\text { GFP-tailswap: androgenic haploid } \\
\text { induction (40\% efficiency) } \\
\quad \text { Gynogenic haploid } \\
\text { induction (5\% efficiency) } \\
\text { DH efficiency } \\
\text { Spontaneous doubling (up to } 100 \%)\end{array}$ & $\begin{array}{l}\text { (Ravi and Chan, 2010; Ravi } \\
\text { et al., 2014) } \\
\text { (Ravi et al., 2014) }\end{array}$ \\
\hline Barley & $x=7$ & $\begin{array}{l}\text { Haploid production } \\
\text { Anther/microspore in vitro culture. } \\
\text { Several Inter-specific crosses } \\
\text { Hap initiator gene (40\% efficiency) } \\
\text { Putative Haploid inducer line } \\
\text { (centromere-mediated genome } \\
\text { elimination) } \\
\text { DH efficiency } \\
70 \%-90 \% \text { (Spontaneous doubling) }\end{array}$ & $\begin{array}{l}\text { (Szarejko, 2003) } \\
\text { (Szarejko, 2003; Sanei et } \\
\text { al., 2011) } \\
\text { (Hagberg and Hagberg, } \\
\text { 1980) } \\
\text { (Karimi-Ashtiyani et al., } \\
\text { 2015) } \\
\text { (Ren et al., 2017) }\end{array}$ \\
\hline Wheat & $3 x=21$ & $\begin{array}{l}\text { Haploid production } \\
\text { In vitro anther culture (plantlets } \\
\text { recovered/100 anthers; average of 5.3, } \\
\text { genotype dependent) } \\
\text { Several inter-specific combinations } \\
\text { DH efficiency } \\
25 \%-75 \% \text { (Spontaneous doubling) }\end{array}$ & $\begin{array}{l}\text { (Lantos et al., 2013) } \\
\text { (Ren et al., 2017) } \\
\text { (Ren et al., 2017) }\end{array}$ \\
\hline Maize & $x=10$ & $\begin{array}{l}\text { Haploid production } \\
\text { Ig W23 - androgenic haploid induction } \\
(3 \%) \\
\text { CENH3-tailswap - gynogenic haploid } \\
\text { induction (up to } 3.6 \%) \\
\text { first division restitution } 1 \text { (fdr1) } \\
\text { MTL gynogenic haploid induction }(6,7 \\
\% \text { efficiency) } \\
\text { Gynogenic haploid induction. Inducer } \\
\text { lines available WS14, RWS, UH400, } \\
\text { BHI306, CAU5 (8\%-10\%) } \\
\text { DH efficiency } \\
9.8 \%-98 \% \text { (Spontaneous doubling) } \\
\text { NO (44\%) }\end{array}$ & $\begin{array}{l}\text { (Kermicle, 1969) } \\
\text { (Kelliher et al., 2016) } \\
\text { (Sugihara et al., 2013) } \\
\text { (Kelliher et al., 2017) } \\
\text { (Ren et al., 2017) } \\
\text { (Wu et al., 2016) } \\
\text { (Kato, 2002) }\end{array}$ \\
\hline Rice & $x=12$ & $\begin{array}{l}\text { Haploid production } \\
\text { In vitro androgenesis } \\
\text { DH efficiency } \\
50 \%-60 \% \text { (Spontaneous doubling) }\end{array}$ & $\begin{array}{l}\text { (Mishra and Rao, 2016) } \\
\text { (Ren et al., 2017) }\end{array}$ \\
\hline
\end{tabular}


2.3 Future pathways for efficient modification of meiosis and possible applications.

Thanks to my results with the model organism Arabidopsis, I anticipate that reverse breeding can now be applied efficiently in crops. Yet, the key for efficient reverse breeding relies on partial suppression of crossover frequencies, instead of complete suppression. The use of VIGS to downregulate COs can always be considered for any crop. VIGS is current the only option to downregulate crossover frequencies in unknown hybrids to generate (near-) homozygous complementing lines. So many different VIGS systems have been described to date that there is no reason not to think of VIGS as a general tool to induce meiotic phenotypes in crops. However, efficient reverse breeding for the generation of mapping populations can also be achieved using stable transformation methods. It is possible that in some species, a VIGS system is not in place and therefore, a stable transformation approach such as RNAi for reverse breeding may be also considered. RNAi technology may be also preferred over VIGS, if a complete CSL panel is the main goal of reverse breeding, as all the possible chromosome combinations - considering there are no incompatibilities - will be eventually obtained. Furthermore, if efficient haploid and double haploid technology is not available, a backcross approach can also be used to obtain CSLs. To avoid lengthy transformation steps with RNAi transgenes of each new genotype that want to be used for subsequent generation of CSLs, an efficient and interesting approach could be a combination of RNAi technology with grafting. A silenced RNAi stock can be maintained over time and used to transmit posttranscriptional silencing to a target plant. Zhang et al 2014 showed that stable hpRNAi::DMC1 transformed Nicotiana tabacum could transmit silencing to wild-type plants through grafting (Zhang et al., 2014). The silencing was successful in male meiosis of the first flower buds, but the data obtained suggested that female gametes remained unaffected. Although the penetrance of the silencing phenotype should be studied in other plant species, and effective grafting technology should exist for the species of choice, these results indicates that the method could be further developed for its application to rapidly generate mapping populations in crop species.

The work described in this thesis shows that a technique is now in place to modify meiosis at will in plants. The large availability of VIGS systems for a vast number of organisms enables the future application of VIGS to modify meiosis also in crops. This, coupled to the increasing knowledge about plant meiosis and the mechanism underlying meiotic recombination indicates that effective control of plant meiosis for breeding purposes is at hand. 


\section{References and notes.}

1. Agüero, J. et al. (2014) 'Effectiveness of gene silencing induced by viral vectors based on Citrus leaf blotch virus is different in Nicotiana benthamiana and citrus plants', Virology. Elsevier, 460-461(July), pp. 154-164. doi: 10.1016/j.virol.2014.04.017.

2. Anderson, L. K. et al. (2003) 'High-resolution crossover maps for each bivalent of Zea mays using recombination nodules.', Genetics, 165(2), pp. 849-865. doi: 10.1073/pnas.95.18.10785.

3. Becker, A. (2013) Virus-Induced Gene Silencing. Methods in Molecular Biology. Edited by A. Becker. Humana Press. doi: 10.1007/978-1-62703-278-0.

4. Becker, A. and Lange, M. (2009) 'VIGS - genomics goes functional', Trends in Plant Science, 15(1), pp. 1-4. doi: 10.1016/j.tplants.2009.09.002.

5. Bennypaul, H. S. et al. (2012) 'Virus-induced gene silencing (VIGS) of genes expressed in root, leaf, and meiotic tissues of wheat.', Functional and Integrative Genomics, 12(1), pp. 143156. doi: 10.1007/s10142-011-0245-0.

6. Bernacki, S. et al. (2010) 'Virus-induced gene silencing as a reverse genetics tool to study gene function.', in Hennig, L. and Köhler, C. (eds) Plant Developmental Biology. Methods and Protocols. Zurich: Humana Press, pp. 27-45. doi: 10.1007/978-1-60761-765-5_3.

7. Bilichak, A. and Kovalchuk, I. (2017) 'Increasing a stable transformation efficiency of Arabidopsis by manipulating the endogenous gene expression using Virus-Induced Gene Silencing.', in Kovalchuk, I. (ed.) Plant epigenetics. Methods in Molecular Biology. Boston, MA: Humana Press, pp. 225-236. doi: 10.1007/978-3-319-55520-1.

8. Bruun-Rasmussen, M. et al. (2007) 'Stability of Barley stripe mosaic virus -induced gene silencing in barley.', Molecular Plant-Microbe Interactions: MPMI, 20(11), pp. 1323-1331. doi: 10.1094/MPMI-20-11-1323.

9. Burch-Smith, T. M. et al. (2004) 'Applications and advantages of virus-induced gene silencing for gene function studies in plants.', Plant Journal, 39(5), pp. 734-746. doi: 10.1111/j.1365313X.2004.02158.x.

10. Burch-Smith, T. M. et al. (2006) 'Efficient virus-linduced gene silencing in Arabidopsis'", Plant Physiology, 142(1), pp. 21-27. doi: 10.1104/pp.106.084624.

11. Chen, Z. J. (2010) 'Molecular mechanisms of polyploidy and hybrid vigor.', Trends in plant sciences, 15(2), pp. 57-71. doi: 10.1016/j.tplants.2009.12.003.Molecular.

12. Chuang, C. F. et al. (1999) 'The PERIANTHIA gene encodes a bZIP protein involved in the determination of floral organ number in Arabidopsis thaliana', Genes and Development, 13(3), pp. 334-344. doi: 10.1101/gad.13.3.334.

13. Couteau, F. et al. (1999) 'Random chromosome segregation without meiotic arrest in both male and female meiocytes of a dmc1 mutant of Arabidopsis.', The Plant Cell, 11(9), pp. 16231634. doi: $10.2307 / 3871042$

14. D'Erfurth, I. et al. (2008) 'Mutations in AtPS1 (Arabidopsis thaliana Parallel Spindle 1) lead to the production of diploid pollen grains.', PLoS Genetics, 4(11), pp. 1-9. doi: 10.1371/journal.pgen.1000274.

15. D'Erfurth, I. et al. (2009) 'Turning meiosis into mitosis.', PLoS Biology, 7(6), p. e1000124. doi: 10.1371/journal.pbio.1000124.

16. Deng, X. et al. (2013) 'Modification of Tobacco rattle virus RNA1 to serve as a VIGS vector reveals that the $29 \mathrm{~K}$ movement protein is an RNA silencing suppressor of the virus.', Molecular Plant-Microbe Interactions: MPMI, 26(5), pp. 503-14. doi: 10.1094/MPMI-12-12-0280-R.

17. Dewitte, A. et al. (2009) 'Occurrence of viable unreduced pollen in a Begonia collection.', Euphytica, 168(1), pp. 81-94. doi: 10.1007/s10681-009-9891-x.

18. Díaz-Camino, C. et al. (2011) 'An effective virus-based gene silencing method for functional 
genomics studies in common bean', Plant Methods, 7(16), pp. 1-11. doi: 10.1186/1746-48117-16.

19. Ding, X. S. et al. (2018) 'An improved Brome mosaic virus silencing vector: Greater insert stability and more extensive VIGS', Plant Physiology, 176(1), pp. 496-510. doi: 10.1104/pp.17.00905.

20. Dion, É. et al. (2007) 'An Arabidopsis MLH1 mutant exhibits reproductive defects and reveals a dual role for this gene in mitotic recombination', Plant Journal, 51(3), pp. 431-440. doi: 10.1111/j.1365-313X.2007.03145.x.

21. Dirks, R. et al. (2009) 'Reverse breeding: A novel breeding approach based on engineered meiosis', Plant Biotechnology Journal, pp. 837-845. doi: 10.1111/j.1467-7652.2009.00450.x.

22. Faivre-Rampant, O. et al. (2004) 'Potato virus $X$-induced gene silencing in leaves and tubers of potato.', Plant physiology, 134(4), pp. 1308-1316. doi: 10.1104/pp.103.037507.

23. Falque, M. et al. (2009) 'Two types of meiotic crossovers coexist in maize', The Plant Cell, 21(12), pp. 3915-3925. doi: 10.1105/tpc.109.071514.

24. Fernandes, J. B. et al. (2017) 'Unleashing meiotic crossovers in hybrid plants', Proceedings of the National Academy of Sciences, 115(10), pp. 2431-2436. doi: 10.1073/pnas.1713078114.

25. Fofana, I. B. F. et al. (2004) 'A geminivirus-induced gene silencing system for gene function validation in cassava', Plant Molecular Biology, 56(4), pp. 613-624. doi: 10.1007/s11103-0040161-y.

26. Galpaz, N. et al. (2006) 'A Chromoplast-Specific carotenoid biosynthesis pathway is revealed by cloning of the tomato white-flower Locus', The Plant Cell, 18(8), pp. 1947-1960. doi: 10.1105/tpc.105.039966.2.

27. Girard, C. et al. (2015) 'AAA-ATPase FIDGETIN-LIKE 1 and helicase FANCM antagonize meiotic crossovers by distinct mechanisms.', PLoS Genetics, 11(7), pp. 1-22. doi: 10.1371/journal.pgen.1005369.

28. Hagberg, A. and Hagberg, G. (1980) 'High frequency of spontaneous haploids in the progeny of an induced mutation in barley', Hereditas, 93(2), pp. 341-343. doi: 10.1111/j.16015223.1980.tb01375.x.

29. Harsant, J. et al. (2013) 'High temperature stress and its effect on pollen development and morphological components of harvest index in the C3 model grass Brachypodium distachyon', Journal of Experimental Botany, 64(10), pp. 2971-2983. doi: 10.1093/jxb/ert142.

30. Heinlein, M. (2015) Plasmodesmata, Methods and Protocols. Edited by M. Heinlein. Hatfield, UK: Humana Press. doi: 10.1007/978-1-4939-1523-1.

31. Higgins, J. D. et al. (2008) 'AtMSH5 partners AtMSH4 in the class I meiotic crossover pathway in Arabidopsis thaliana, but is not required for synapsis', The Plant Journal, 55(1), pp. 28-39. doi: 10.1111/j.1365-313X.2008.03470.x.

32. Holzberg, S. et al. (2002) 'Barley strip mosaic virus-induced gene silencing in a monocot plant.', The Plant Journal, 30(3), pp. 315-327. doi: 10.1046/j.1365-313X.2002.01291.x.

33. Da Ines, O. et al. (2013) 'Meiotic recombination in Arabidopsis Is catalysed by DMC1, with RAD51 playing a supporting role', PLoS Genetics, 9(9), p. e1003787. doi: 10.1371/journal.pgen.1003787.

34. Juvale, P. S. et al. (2012) 'Temporal and spatial Bean pod mottle virus-induced gene silencing in soybean.', Molecular Plant Pathology, 13(9), pp. 1140-1148. doi: 10.1111/j.13643703.2012.00808.x.

35. Kalantidis, K. et al. (2008) 'RNA silencing movement in plants.', Biology of the Cell. Mello and Conte, 100(1), pp. 13-26. doi: 10.1042/BC20070079.

36. Karimi-Ashtiyani, R. et al. (2015) 'Point mutation impairs centromeric CENH3 loading and 
induces haploid plants', Proceedings of the National Academy of Sciences. National Academy of Sciences, 112(36), pp. 11211-11216. doi: 10.1073/pnas.1504333112.

37. Kato, A. (2002) 'Chromosome doubling of haploid maize seedlings using nitrous oxide gas at the flower primordial stage', Plant Breeding, 121(5), pp. 370-377. doi: 10.1046/j.14390523.2002.743321.x.

38. Kelliher, T. et al. (2016) 'Maternal haploids are preferentially induced by CENH3-tailswap transgenic complementation in maize.', Frontiers in plant science, 7 (March), p. 414. doi: 10.3389/fpls.2016.00414.

39. Kelliher, T. et al. (2017) 'MATRILINEAL, a sperm-specific phospholipase, triggers maize haploid induction', Nature., 542(7639), pp. 105-109. doi: 10.1038/nature20827.

40. Kermicle, J. L. (1969) 'Androgenesis conditioned by a mutation in maize', Science, 166(3911), pp. $1422-1424$.

41. Kundu, L. M. and Ray, S. (2017) 'Mitotic abnormalities and micronuclei inducing potentials of colchicine and leaf aqueous extracts of Clerodendrum viscosum Vent. in Allium cepa root apical meristem cells', Caryologia, 70(1), pp. 7-14.

42. Lacomme, C., Hrubikova, K. and Hein, I. (2003) 'Enhancement of virus-induced gene silencing through viral-based production of inverted-repeats', The Plant Journal, 34(4), pp. 543-553. doi: 10.1046/j.1365-313X.2003.01733.x.

43. Lambing, C., Franklin, F. C. H. and Wang, C.-J. R. (2017) 'Understanding and manipulating meiotic recombination in plants.', Plant Physiology, 173(3), pp. 1530-1542. doi: 10.1104/pp.16.01530.

44. Lantos, C. et al. (2013) 'Efficient application of in vitro anther culture for different European winter wheat (Triticum aestivum L.) breeding programmes', Plant Breeding, 132(2), pp. 149154. doi: 10.1111/pbr.12032.

45. Li, X., Li, L. and Yan, J. (2015) 'Dissecting meiotic recombination based on tetrad analysis by single-microspore sequencing in maize.', Nature Communications. 6(6648), pp. 1-9. doi: 10.1038/ncomms7648.

46. Lim, K. B. et al. (2001) 'Indeterminate meiotic restitution (IMR): A novel type of meiotic nuclear restitution mechanism detected in interspecific lily hybrids by GISH', Theoretical and Applied Genetics, 103(2-3), pp. 219-230. doi: 10.1007/s001220100638.

47. Lim, K. B. et al. (2004) 'Occurrence of SDR $2 \mathrm{~N}$-gametes in Lilium hybrids', Breeding Science, 54(1), pp. 13-18. doi: Doi 10.1270/Jsbbs.54.13.

48. Liu, E. and Page, J. E. (2008) 'Optimized cDNA libraries for virus-induced gene silencing (VIGS) using Tobacco rattle virus', Plant Methods, 4(5). doi: 10.1186/1746-4811-4-5.

49. Liu, Y. L., Schiff, M. and Dinesh-Kumar, S. P. (2002) 'Virus-induced gene silencing in tomato', The Plant Journal, 31(6), pp. 777-786. doi: 10.1111/j.1365-313X.2005.02441.x.

50. Mason, A. S. et al. (2011) 'Production of viable male unreduced gametes in Brassica interspecific hybrids is genotype specific and stimulated by cold temperatures', BMC Plant Biology, 11(103). doi: 10.1186/1471-2229-11-103.

51. Mei, Y. and Whitham, S. A. (2018) 'Virus-induced gene silencing in maize with a Foxtail mosaic virus vector.', Methods in Molecular Biology, 1676, pp. 129-139. doi: 10.1007/978-1-49397315-6_7.

52. Mieulet, D. et al. (2016) 'Turning rice meiosis into mitosis', Cell Research., 26(11), pp. 12421254. doi: $10.1038 / \mathrm{cr} .2016 .117$.

53. Mishra, R. and Rao, G. J. N. (2016) 'In-vitro Androgenesis in rice: advantages, constraints and future prospects', Rice Science, 23(2), pp. 57-68. doi: 10.1016/j.rsci.2016.02.001.

54. O'Keeffe, K. (2009) Maize growth \& development. Edited by J. Edwards. Procrop. Available at: www.dpi.nsw.gov.au. 
55. Pacak, A. et al. (2010) 'Investigations of Barley stripe mosaic virus as a gene silencing vector in barley roots and in Brachypodium distachyon and oat', Plant methods, 6(26), pp. 1-16.

56. Padmanabhan, M. and Dinesh-Kumar, S. P. (2009) 'Virus-induced gene silencing as a tool for delivery of dsRNA into plants', Cold Spring Harbor Protocols, 4(2). doi: 10.1101/pdb.prot5139.

57. Pang, J. et al. (2013) 'Development of Agrobacterium-mediated virus-induced gene silencing and performance evaluation of four marker genes in Gossypium barbadense.', PLOS ONE, 8(9), p. e73211. doi: 10.1371/journal.pone.0073211.

58. Peele, C. et al. (2001) 'Silencing of a meristematic gene using geminivirus-derived vectors', The Plant Journal, 27(4), pp. 357-366. doi: 10.1046/j.1365-313X.2001.01080.x.

59. Pflieger, S. et al. (2008) 'Efficient virus-induced gene silencing in Arabidopsis using a "onestep" TYMV-derived vector', Plant Journal, 56(4), pp. 678-690. doi: 10.1111/j.1365313X.2008.03620.x.

60. Pflieger, S. et al. (2014) 'The "one-step" Bean pod mottle virus (BPMV)-derived vector is a functional genomics tool for efficient overexpression of heterologous protein, virus-induced gene silencing and genetic mapping of BPMV R-gene in common bean (Phaseolus vulgaris L.)', BMC Plant Biology, 14(232), pp. 1471-2229. doi: 10.1186/s12870-014-0232-4.

61. Prusicki, M. A. et al. (2018) 'Live cell imaging of meiosis in Arabidopsis thaliana - a landmark system', bioRxiv. doi: 10.1101/446922.

62. Pyott, D. E. and Molnar, A. (2015) 'Going mobile: Non-cell-autonomous small RNAs shape the genetic landscape of plants', Plant Biotechnology Journal, 13(3), pp. 306-318. doi: 10.1111/pbi.12353.

63. Quadrana, L. et al. (2011) 'Coupling virus-induced gene silencing to exogenous green fluorescence protein expression provides a highly efficient system for functional genomics in Arabidopsis and across all stages of tomato fruit development.', in Plant Physiology, pp. 12781291. doi: 10.1104/pp.111.177345.

64. Ravi, M. et al. (2014) 'A haploid genetics toolbox for Arabidopsis thaliana', Nature Communications., 5(5334). doi: 10.1038/ncomms6334.

65. Ravi, M. and Chan, S. W. L. (2010) 'Haploid plants produced by centromere-mediated genome elimination', Nature, 464(7288), pp. 615-618. doi: 10.1038/nature08842.

66. Ren, J. et al. (2017) 'Novel technologies in doubled haploid line development', Plant Biotechnology Journal, 15(11), pp. 1361-1370. doi: 10.1111/pbi.12805.

67. Rodriguez, E. M. et al. (2001) 'Colchicine promotes a change in chromosome structure without loss of sister chromatid cohesion in prometaphase l-arrested bivalents.', Chromosoma., 110(7), pp. 478-486. doi: 10.1007/s004120100162.

68. Ruiz, M. T., Voinnet, O. and Baulcombe, D. C. (1998) 'Initiation and maintenance of virusinduced gene silencing.', The Plant Cell, 10(6), pp. 937-946.

69. Sanei, M. et al. (2011) 'Loss of centromeric histone H3 (CENH3) from centromeres precedes uniparental chromosome elimination in interspecific barley hybrids, 108(33) E498-505, doi: 10.1073/pnas.1103190108.

70. Sattler, M. C., Carvalho, C. R. and Clarindo, W. R. (2016) 'The polyploidy and its key role in plant breeding', Planta., 243(2), pp. 281-296. doi: 10.1007/s00425-015-2450-x.

71. Schwartz, D. and Osterman, J. (1976) 'A pollen selection system for alcohol-dehydrogenase negative mutants in plants', Genetics, 83(1), pp. 63-65.

72. Séguéla-Arnaud, M. et al. (2015) 'Multiple mechanisms limit meiotic crossovers: TOP3a and two BLM homologs antagonize crossovers in parallel to FANCM', Proceedings of the National Academy of Sciences, 112(15), pp. 4713-4718. doi: 10.1073/pnas.1423107112.

73. Séguéla-Arnaud, M. et al. (2017) 'RMI1 and TOP3a limit meiotic CO formation through their C-terminal domains', Nucleic Acids Research, 45(4), pp. 1860-1871. doi: 
10.1093/nar/gkw1210.

74. Senthil-Kumar, M. and Mysore, K. S. (2011) 'New dimensions for VIGS in plant functional genomics', Trends in Plant Science. Elsevier Ltd, 16(12), pp. 656-665. doi: 10.1016/j.tplants.2011.08.006.

75. Senthil-Kumar, M. and Mysore, K. S. (2014) 'Tobacco rattle virus-based virus-induced gene silencing in Nicotiana benthamiana', Nature Protocols, 9(7), pp. 1549-1562. doi: 10.1038/nprot.2014.092.

76. Sidhu, G. K., Warzecha, T. and Pawlowski, W. P. (2017) 'Evolution of meiotic recombination genes in maize and teosinte', BMC Genomics., 18(106), pp. 1-17. doi: 10.1186/s12864-0173486-z.

77. De Storme, N., Copenhaver, G. P. and Geelen, D. (2012) 'Production of diploid male gametes in Arabidopsis by cold-induced destabilization of postmeiotic radial microtubule arrays', Plant Physiology, 160(4), pp. 1808-1826. doi: 10.1104/pp.112.208611.

78. De Storme, N. and Geelen, D. (2011) 'The Arabidopsis mutant jason produces unreduced first division restitution male gametes through a parallel/fused spindle mechanism in meiosis II', Plant Physiology, 155(3), pp. 1403-1415. doi: 10.1104/pp.110.170415.

79. Sugihara, N. et al. (2013) 'Haploid plants carrying a sodium azide-induced mutation (fdr1) produce fertile pollen grains due to first division restitution (FDR) in maize (Zea mays L.)', Theoretical and Applied Genetics, 126(12), pp. 2931-2941. doi: 10.1007/s00122-013-21839.

80. Susi, H. et al. (2015) 'Co-infection alters population dynamics of infectious disease', Nature Communications, 6(5975). doi: 10.1038/ncomms6975.

81. Susi, P. et al. (2004) 'Characteristics of RNA silencing in plants: similarities and diferences across kingdoms', Plant Molecular Biology, 54(2), pp. 157-174.

82. Szarejko, I. (2003) 'Anther culture for doubled haploid production in barley (Hordeum vulgare L.)', in Maluszynski, M. et al. (eds) Doubled Haploid Production in Crop Plants. Dordrecht: Springer, pp. 35-42. doi: 10.1007/978-94-017-1293-4_6.

83. Tanaka, Y., Sasaki, N. and Ohmiya, A. (2008) 'Biosynthesis of plant pigments: anthocyanins, betalains and carotenoids', The Plant Journal, 54(4), pp. 733-749. doi: 10.1111/j.1365313X.2008.03447.X.

84. Tao, X. and Zhou, X. (2004) 'A modified viral satellite DNA that suppresses gene expression in plants', The Plant Journal, 38(5), pp. 850-860. doi: 10.1111/j.1365-313X.2004.02087.x.

85. Tian, J. et al. (2014) 'TRV-GFP: A modified Tobacco rattle virus vector for efficient and visualizable analysis of gene function', Journal of Experimental Botany, 65(1), pp. 311-322. doi: $10.1093 / \mathrm{j} \times \mathrm{b} / \mathrm{ert3} 31$.

86. Tonosaki, K. et al. (2018) 'Overcoming the species hybridization barrier by ploidy manipulation in the genus Oryza', The Plant Journal, 93(3), pp. 534-544. doi: 10.1111/tpj.13803.

87. Turnage, M. A. et al. (2002) 'Geminivirus-based vectors for gene silencing in Arabidopsis', The Plant Journal, 30(1), pp. 107-114. doi: 10.1046/j.1365-313X.2002.01261.x.

88. Tuttle, J. R. et al. (2008) 'Geminivirus-mediated gene silencing from Cotton leaf crumple virus is enhanced by low temperature in cotton', Plant Physiology, 148(1), pp. 41-50. doi: 10.1104/pp.108.123869.

89. Van Tuyl, J. M. et al. (2005) 'Meiotic polyploidization in five different interspecific Lilium hybrids', Acta Horticulturae, 673, pp. 99-105. doi: 10.17660/ActaHortic.2005.673.10.

90. Ueki, S. and Citovsky, V. (2005) 'Control improves with age: Intercellular transport in plant embryos and adults', Proceedings of the National Academy of Sciences, 102(6), pp. 18171818.

91. Vaghchhipawala, Z. et al. (2011) 'Agroinoculation and Agroinfiltration: Simple tools for 
complex gene function analyses.', in A., P. (ed.) Plant reverse genetics. Methods in Molecular Biology. Humana Press, Totowa, NJ, pp. 65-76. doi: 10.1007/978-1-60761-682-5.

92. Wang, J. et al. (2017) 'High temperature-induced production of unreduced pollen and its cytological effects in Populus', Scientific Reports. Springer US, 7(5281). doi: 10.1038/s41598017-05661-x.

93. Wang, R. et al. (2016) 'An efficient virus-induced gene silencing vector for maize functional genomics research', The Plant Journal, 86(1), pp. 102-115. doi: 10.1111/tpj.13142.

94. Wijnker, E. et al. (2012) 'Reverse breeding in Arabidopsis thaliana generates homozygous parental lines from a heterozygous plant', Nature Genetics, 44(4), pp. 467-470. doi: 10.1038/ng.2203.

95. Wijnker, E. and de Jong, H. (2008) 'Managing meiotic recombination in plant breeding', Trends in Plant Science, 13(12), pp. 640-646. doi: 10.1016/j.tplants.2008.09.004.

96. Wu, C., Lingling, J. and Goggin, F. (2011) 'The reliability of virus-induced gene silencing experiments using Tobacco rattle virus in tomato is influenced by the size of the vector control', Molecular Plant Pathology, 12(3), pp. 299-305. doi: 10.1111/j.1364-3703.2010.00669.x.

97. Wu, P. et al. (2016) 'New Insights into the genetics of haploid male fertility in maize', Crop Science. Madison, WI: The Crop Science Society of America, Inc., 57(2), pp. 637-647. doi: 10.2135/cropsci2016.01.0017.

98. Younis, A., Hwang, Y.-J. and Lim, K.-B. (2014) 'Exploitation of induced 2n-gametes for plant breeding', Plant Cell Reports, 33(2), pp. 215-223. doi: 10.1007/s00299-013-1534-y.

99. Zhang, P. et al. (2017) 'The rice AAA-ATPase OsFIGNL1 Is essential for male meiosis', Frontiers in Plant Science, 8(1639), pp. 1-17. doi: 10.3389/fpls.2017.01639.

100. Zhang, W. et al. (2014) 'Graft-transmissible movement of inverted-repeat-induced siRNA signals into flowers', The Plant Journal, 80(1), pp. 106-121. doi: 10.1111/tpj.12622. 
To the end of the journey,

\author{
"Una caja de recuerdos \\ y fiestas de guardar media vida en cada intento \\ y la otra media en pinzas de metal ... \\ ... Fue tan largo el duelo que al final, \\ casi lo confundo con mi hogar."
}

Cuarteles de invierno, Vetusta Morla. 


\section{Summary.}

Plant breeders produce the best possible plant varieties by crossing and subsequently selecting for desirable phenotypes, that arise from new allele combinations in the offspring. The generation of new allelic combinations is controlled by the process of meiosis: a specialized cell division in which four haploid gametes are formed from a diploid mother cell. There are two special events that take place in meiosis. First, due to crossover recombination, genetic information between homologous chromosomes is exchanged, hence creating new allelic combinations. The process of crossover recombination is followed by two consecutive cell divisions. During the first meiotic division, recombinant homologous chromosomes segregate. Afterwards, the two chromatids of each chromosome segregate during the second meiotic divisions to form haploid gametes carrying new combinations of recombinant chromosomes. Among all the possible offspring that can be generated using these gametes, only certain allelic combinations may be wanted from a breeder's point of view, as only specific phenotypes may be desirable. Tight control over the process of meiotic recombination allows plant breeders to promote or maintain certain allelic combinations that can desirable. For instance, an increase of crossover frequencies boosts homologous recombination between regions that usually do not recombine. On the other hand, if the idea is to preserve beneficial allelic combinations present in a specific plant, a decrease of crossover rates is preferred. In addition, the reduction of the number of meiotic divisions can be used to generate diploid gametes instead of haploid gametes and thus, obtain plants with a higher ploidy level: polyploids. Polyploids are important resources for plant breeders as they can display bigger organs than the diploid counterparts, which for instance it is of major importance in the field of flower breeders. Another example is the use of triploids in commercial breeding; due to the acute sterility displayed by triploids plants, they are currently used to produce fruits without seeds, like in the case of watermelon or banana.

Different genes control each of these processes in meiosis and breeders usually need to rely on mutations or stable silencing techniques such as RNAi to obtain a meiotic phenotype. However, these approaches are usually lengthy and/or require a step of plant stable transformation, a process that many plant species are recalcitrant to. Plant breeders would like to control meiosis at will, with simple tools, for example using a rapid and transient silencing approach like virus-induced gene silencing (VIGS). A VIGSinduced knockdown is achieved by inoculating the plant with an engineered virus that contains in its genome a sequence homologous to the plant target gene. The presence of the virus in the plant triggers the activation of the immune system that ultimately degrades the virus and the endogenous plant mRNA homologous to the target sequence cloned in the virus. VIGS silencing mechanism is rapid (can induce a phenotype in few days after treatment) and it is often not transmitted to the offspring. In case it is transmitted, the offspring can be selected against the presence or the virus, or the virus 
can be inactivated using different methods, such us cold-shock. VIGS-systems are available for a wide range of plant species, including those that are so far, recalcitrant to stable transformation methods.

In this thesis, I have established and optimized VIGS as a technique to induce several meiotic phenotypes in the plant model organism Arabidopsis thaliana. I thereby focus on those that are interesting to further develop breeding applications: decrease and increase of crossover frequencies and the modification of meiosis to produce diploid gametes. To exploit the use of VIGS for meiosis, in Chapter 2 several meiotic phenotypes were induced. For instance, I could phenocopy the phenotypes of mutants that display a complete absence of crossover formation and sister-chromatid cohesion. In addition, I induce diploid gamete formation by downregulating an essential gene at the onset of second meiotic division. This lead to the generation of tetraploid offspring in four distinct accession of Arabidopsis thaliana. Finally, I also targeted two post-meiotic genes that are required for pollen separation, obtaining pollen tetrads instead of independent pollen grains, a phenotype that can used a selection marker. Chapter 3 explores the use of VIGS coupled to a breeding application, reverse breeding. Reverse breeding benefits from low crossover rates to generate parental lines for a heterozygous plants. VIGS was used in a reverse breeding experimental set-up to decrease crossover frequencies by $80 \%$ in Arabidopsis hybrids and to offspring with non-recombinant and low-recombinant chromosomes. On the other hand, in Chapter 4 VIGS was used to upregulate meiotic recombination frequencies. It has been shown that mutants for different negative regulators of crossover formation displayed a significant increase of crossover frequencies compared to wild-type plants. Two of these genes were targeted in Arabidopsis plants using VIGS and cytological analysis of chromosomes during the stages in which crossover recombination occurs revealed that the pairing configuration is consistent with an increase of crossover numbers. However, offspring with increased $\mathrm{CO}$ events could not be recovered, suggesting that the VIGS-mediated knockdown of these genes hampers the formation of viable gametes or causes other developmental defects. Chapter 5 presents a step-by-step protocol of how to use VIGS to silence meiotic genes in Arabidopsis thaliana. The protocol includes an overview of VIGS construct cloning and detailed guidelines for the experimental design. Because the virus is active in the plant for a limited amount of time, this protocol specially highlights the importance of maintaining the balance between the natural lifespan of the virus throughout the critical developmental stages in the plant, to ultimately, guarantee successful silencing in meiosis. The protocol also describes the validation of meiotic phenotypes. Lastly Chapter 6 discusses first the possibilities and limitations of VIGS as a universal tool to modify meiosis in plants and the adaptation of the VIGS systems for different plant species. In the second part of this chapter it is discussed how reverse breeding through partial decrease of crossover frequencies can be pursued to efficiently obtain offspring in high chromosome number species. 


\section{Samenvatting.}

Plantenveredelaars produceren de best mogelijke plantenvariëteiten door planten te kruisen en vervolgens onder de nakomelingen te selecteren op gewenste fenotypes. Die fenotypes zijn het gevolg van nieuwe combinaties van allelen die hun oorsprong vinden in de meiose: een gespecialiseerde celdeling waarin vier haploide sporen ontstaan uit een diploide moedercel. Tijdens de meiose vinden er vervolgens twee belangrijke processen plaats. Het eerste is de vorming van nieuwe allelcombinaties doordat de twee homologen van elk chromosoom onderling corresponderende stukken DNA uitwisselen. Dit proces staat bekend als crossing over. Een tweede belangrijk proces is de uitvoering van twee achtereenvolgende celdelingen. Tijdens de eerste meiotische deling segregeren de homologe chromosomen, en in de tweede meiotische deling segregeren de chromatiden waardoor vier haploide gameten gevormd worden net nieuwe combinaties van recombinante chromosomen. Tussen alle mogelijke nakomelingen die uit deze gameten gevormd kunnen worden, is er slechts een beperkt aantal dat -vanuit het perspectief van de veredelaar- interessant is, omdat veredelaars alleen in heel specifieke combinaties van eigenschappen geïnteresseerd zijn. Wanneer plantenveredelaars controle hebben over het proces van meiotische recombinatie, kunnen zij zowel het ontstaan van nieuwe combinaties van allelen bevorderen alsook het behouden van interessante allel combinaties. Een verhoging van bijvoorbeeld het aantal crossing overs verhoogt de kans dat men nieuwe combinaties van allelen vindt op stukken van chromosomen waar crossing over normaliter zeldzaam is. Wanneer een bepaalde plant echter unieke, waardevolle allelcombinaties bezit, kan het voorkomen van crossing over ervoor zorgen dat die gewilde combinaties van allelen in de gameten behouden blijven. Het is daarnaast ook mogelijk om het aantal meiotische celdelingen te beperken: wanneer er maar één in plaats van twee celdelingen plaatsvinden, ontstaan er diploide in plaats van haploide gameten: gameten met tweemaal zoveel chromosomen. Daarmee kunnen polyploide nakomelingen worden gemaakt: planten met meer dan twee kopieen van elk chromosoom. Voor de veredeling kunnen polyploiden zeer interessante planten zijn omdat zij bijvoorbeeld grotere bloemen vormen: een belangrijke eigenschap in de sierbloementeelt. Een andere interessante eigenschap van polyploiden is dat zij vaak minder- of niet vruchtbaar zijn en daarmee gebruikt kunnen worden voor de productie van vruchten zonder zaden zoals bij bananen of zaadloze watermeloenen.

Specifieke genen zijn belangrijk voor deze processen tijdens de meiose, en wanneer plantenveredelaars meiotische processen willen beïnvloeden, moeten zij gebruik maken van genmutaties of transgene technieken zoals het gebruik van RNAi constructen om een bepaald (mutant-) meiotisch fenotype te induceren. Het vinden van mutanten is soms niet eenvoudig maar ook het proces van stabiele transformatie kan lang duren, of is voor sommige soorten helemaal niet mogelijk. Plantenveredelaars hebben echter behoefte aan eenvoudige methoden waarmee de verschillende meiotische processen 
eenvoudig te sturen zijn. Het gebruik van virus-geïnduceerde gen suppressie (VIGS) is een mogelijke methode voor het tijdelijk uitschakelen van een bepaalde genfunctie. Het uitschakelen van een gen door middel van VIGS is het gevolg van de inoculatie van een plant met een genetisch gemodificeerd virus. In dat virus is een gensequentie ingebracht van het gen dat (tijdelijk) uitgeschakeld moet worden. De inoculatie van een plant met het gemodificeerde virus leidt tot de activatie van het immuunsysteem van de plant dat via verschillende stappen uiteindelijk het virus afbreekt, maar ook het plant-eigen mRNA van het gen waarvan je de expressie wilt stoppen. De toepassing van VIGS is snel (kan al in enkele dagen leiden tot het gewilde fenotype in een plant) en het virus wordt vaak niet doorgegeven naar de nakomelingen. In het geval het virus wel in de nakomelingen terecht komt, kan ertegen worden geselecteerd of het virus kan geïnactiveerd worden, bijvoorbeeld door een koude behandeling. VIGS methodes zijn beschikbaar voor een veelheid aan plantensoorten, waarbij ook vele waarvoor geen transformatie protocollen beschikbaar zijn.

In dit proefschrift ontwikkel en optimaliseer ik VIGS als een techniek voor het induceren van een verscheidenheid aan (mutant-) meiotische fenotypes in de modelplant Arabidopsis thaliana. Ik richt mij daarbij met name op die fenotypes die mogelijk belangrijk zijn in de ontwikkeling van toepassingen in de plantenveredeling: de verlaging- en verhoging van crossing over tijdens de meiose en het beïnvloeden van een van de meiotische celdelingen waardoor er diploiden gameten ontstaan.

In hoofdstuk 2 beschrijf ik verschillende meiotische fenotypes die via VIGS geïnduceerd konden worden. Het lukte bijvoorbeeld om middels VIGS de expressie van genen die belangrijk zijn crossing over of cohesie tussen zuster-chromatiden tijdelijk uit te schakelen. Daarbij lieten de planten een vergelijkbaar fenotype zien als de mutanten van die meiotische genen. Ook laat ik zien dat diploide gameten gevormd kunnen worden door met VIGS een gen uit te schakelen dat essentieel is voor de tweede meiotische deling. Ik laat zien dat dit in vier verschillende Arabidopsis lijnen leidt tot de vorming van tetraploide nakomelingen. Ten slotte laat ik zien dat een verminderde expressie van twee genen die belangrijk zijn voor het losraken van pollen, leidt tot de vorming van zogenaamde "pollen tetraden", waarbij de vier stuifmeelkorrels die uit een meiose gevormd worden aan elkaar blijven plakken. Dat phenotype kan gebruikt worden als selectie-merker. In hoofdstuk 3 koppel ik het gebruik van VIGS aan de ontwikkeling van een veredelingstechniek voor planten, wel bekend als "reverse breeding". Reverse breeding is een techniek waarbij de verlaging van crossing over in een heterozygote plant leidt tot het verkrijgen van ouderlijnen voor die plant. Met behulp van VIGS werd crossing over met $80 \%$ verlaagd in een heterozygote plant, waarna nakomelingen verkregen werden met geen- of weinig crossing over. De verhoging van crossing over door middel van het gebruik van VIGS is het onderwerp van hoofdstuk 4. In planten zijn genen bekend die crossing over onderdrukken en waarvan de mutanten verhoogde aantallen crossing overs laten zien. De expressie van twee van die genen werd met 
VIGS onderdrukt, waarna een cytologische analyse van de meiotische chromosomen liet zien dat de behandeling met VIGS inderdaad leidt tot een chromosoombeeld dat hoort bij verhoogde crossing over. Echter, onder de nakomelingen werden geen planten gevonden met verhoogde crossing over, wat doet vermoeden dat het uitschakelen van deze genen via VIGS tot onbedoelde bij-effecten leidt zoals steriliteit of andere ontwikkelingsproblemen. Tenslotte in hoofdstuk 5 beschrijf ik een protocol waarin stap voor stap wordt uitgelegd hoe de expressie van meiotische genen kan worden verhinderd met VIGS in Arabidopsis thaliana. Het protocol beschrijft belangrijke elementen in de klonering van VIGS constructen en geeft gedetailleerd aan hoe experimenten opgezet dienen te worden. Omdat het virus maar een bepaalde tijd actief is in de plant, legt het protocol de nadruk op het precies laten samenvallen van het tijdstip van meiose en de activiteit van het virus om de expressie van meiotische genen succesvol te onderdrukken. Het protocol beschrijft ook hoe de gevonden fenotypes gevalideerd kunnen worden. In hoofdstuk 6 worden eerst de mogelijkheden maar ook de beperkingen beschreven voor de toepassing van VIGS als een breed inzetbare methode voor het veranderen van meiotische processen in verschillende plantensoorten. In het tweede deel wordt de toepassing van reverse breeding in gewassen besproken, waarbij de vraag centraal staat of een incomplete onderdrukking van crossing over tot een efficiënte methode kan leiden voor de toepassing van reverse breeding in gewassen met hoge chromosoom-aantallen. 


\section{Resumen.}

Los fitomejoradores producen las mejores variedades comerciales mediante cruzamientos y posterior selección de fenotipos interesantes, los cuales vienen determinados por combinaciones alélicas especificas que se dan en la descendencia. La generación de nuevas combinaciones alélicas está controlada por el proceso de meiosis: una división celular especializada en la que una célula madre diploide da lugar cuatro gametos haploides. La meiosis cuenta con dos procesos característicos. Primero, gracias al proceso de recombinación homóloga, los cromosomas homólogos intercambian información genética, creando de esta manera nuevas combinaciones de alelos. Inmediatamente tras la recombinación homóloga, tienen lugar dos divisiones celulares consecutivas. Durante la primera división meiótica, los cromosomas homólogos recombinantes se separan. Seguidamente, durante la segunda división meiótica, las dos cromátidas hermanas segregan para formar gametos haploides que contienen nuevas combinaciones de cromosomas recombinantes. De la posible descendencia generada utilizando estos gametos, solamente ciertas combinaciones alélicas -que corresponden con fenotipos específicos- pueden ser interesantes desde el punto de vista de un fitomejorador. Ser capaz de controlar el proceso de meiosis hace que los fitomejoradores puedan mantener o, por el contrario, promover la formación de combinaciones alélicas deseables. Por ejemplo, un incremento en la frecuencia de recombinación implica que el intercambio genético ocurra entre regiones en el cromosoma que normalmente no recombinan. Sin embargo, para conservar combinaciones alélicas beneficiosas es necesario reducir la frecuencia de recombinación que tiene lugar durante meiosis. Asimismo, la reducción en el número de divisiones meióticas puede ser empleado para generar gametos diploides -en vez de gametos haploides- y por lo tanto obtener plantas que tengan un nivel de ploidía superior, las cuales reciben el nombre de poliploides. Plantas poliploides representan un recurso importante para los fitomejoradores, ya que en general presentan un incremento en el tamaño de ciertos órganos comparados con su equivalente diploide, lo cual representa una ventaja sustancial, por ejemplo, en la producción comercial de flores. Otro ejemplo es el uso de triploides en el ámbito comercial: Debido a la severa infertilidad característica de plantas triploides, estas se utilizan para producir frutas que carecen de semillas, como en el caso de la sandia o la banana.

Distintos genes controlan cada uno de los procesos previamente mencionados en meiosis y los fitomejoraders normalmente emplean como principal recurso mutantes en los genes de interés o, por el contrario, técnicas de silenciamiento génico como RNAi para inducir un fenotipo mutante. Sin embargo, estos métodos requieren tiempo y/o un paso intermedio de transformación estable de la planta, un proceso al cual muchas plantas son recalcitrantes. A los fitomejoradores les interesa ser capaces de controlar meiosis a voluntad, empleando técnicas sencillas de silenciamiento génico que sean rápidas y temporales, como por ejemplo con un método denominado silenciamiento 
génico inducido por virus o también denominado VIGS (de sus siglas en inglés: virusinduced gene silencing). Un knockdown inducido por VIGS se consigue mediante la inoculación en la planta de un virus modificado para portar una secuencia homóloga al gen de interés que se va a silenciar. La presencia del virus en la planta activa su sistema inmune, generando así una respuesta que terminará degradando el virus y a su vez el ARN mensajero endógeno que comparte homología con la secuencia que se había insertado en el genoma vírico. VIGS es un mecanismo de silenciamiento génico rápido (puede inducir un fenotipo en pocos días tras el tratamiento) y en general no se transmite a la descendencia. En caso de que esto último suceda, la descendencia que no contenga el virus puede ser seleccionada, o el virus puede ser desactivado mediante diferentes estrategias como, por ejemplo, utilizando un shock frio. Los sistemas víricos asociados a VIGS se encuentran disponibles para ser utilizados en un gran número de especies, incluyendo aquellas que son recalcitrantes a métodos estables de transformación.

En esta tesis he establecido y optimizado VIGS para inducir varios fenotipos mutantes en miosis en el organismo modelo Arabidopsis thaliana. En concreto, me centro en aquellos que son interesantes para el futuro desarrollo de aplicaciones que sean útiles desde el punto de vista de la fitomejoración, siendo: la disminución o aumento de frecuencias de recombinación homóloga y la modificación del número de divisiones meióticas para producir gametos diploides. Para ilustrar como se puede explotar el uso de VIGS para modificar meiosis, en el Capítulo 2 varios fenotipos mutantes en meiosis fueron inducidos. Por ejemplo, pude obtener el mismo fenotipo en meiosis que mutantes previamente descritos en el caso de pérdida completa de recombinación genética y también en el caso de pérdida de cohesión entre cromátidas hermanas. Igualmente, pude inducir la formación de gametos diploides tras el silenciamiento de un gen que codifica para una proteína cuya actividad se requiere durante la segunda división meiótica. Esto dio lugar a la obtención de descendencia tetraploide en cuatro ecotipos distintos de Arabidopsis thaliana. Finalmente, también silencié dos genes con función post-meiótica, los cuales son necesarios para la separación de los granos de polen, lo cual causó que los cuatro granos de polen producto de una meiosis se liberen en forma de tétradas. Este fenotipo puede ser utilizado en el futuro como marcador fenotípico en experimentos con VIGS. En el Capítulo 3 se explora la aplicación de VIGS expresamente en una técnica de fitomejoración, denominada reverse breeding. Reverse breeding funciona a través de una reducción en las frecuencias de recombinación en una planta heterocigota para producir líneas parentales para esta planta. En este caso, VIGS fue empleado para reducir en un $80 \%$ la frecuencia de recombinación en plantas híbridas de Arabidopsis y así, obtener descendencia con cromosomas no-recombinantes y con recombinación baja. Por el contrario, en el Capítulo 4, VIGS se utilizó para aumentar la frecuencia de recombinación homóloga. Se ha demostrado que diferentes mutantes que carecen de la función de genes 
implicados en regular negativamente la recombinación, muestran un incremento significante de las frecuencias totales de recombinación comparadas con plantas wildtype. Dos de estos reguladores negativos fueron silenciados en Arabidopsis mediante VIGS. Análisis citológicos de los cromosomas que se encuentran en meiosis durante el periodo en el que la recombinación homóloga tiene lugar, revelaron que la configuración durante el apareamiento es consistente con un aumento del numero total de quiasmas. Sin embargo, no pude recuperar descendencia con un incremento en el número de eventos de recombinación, lo que sugiere que el silenciamiento de estos genes causado por VIGS impide la formación de gametos viables o da lugar a otros defectos durante el desarrollo. En el Capítulo 5 se introduce un protocolo detallado de cómo emplear VIGS para silenciar genes meióticos en Arabidopsis thaliana. El protocolo incluye una visión general de cómo producir un sistema VIGS e instrucciones detalladas para llevar acabo el diseño experimental. Dado que el virus es activo en la planta durante un periodo de tiempo limitado, este protocolo está ideado para garantizar la actividad del virus desde el momento de inoculación hasta los momentos del desarrollo críticos en la planta y así observar un fenotipo en meiosis. La validación de estos fenotipos también se explica en el protocolo. Por último, en el Capítulo 6 se exponen las posibilidades y limitaciones de VIGS como una herramienta universal para modificar meiosis en plantas, así como la adaptación de sistemas VIGS a diferentes especies. En la segunda parte de este capítulo y se considera el potencial uso de reverse breeding en plantas más complejas, evaluando si reverse breeding mediante la reducción parcial de frecuencias de recombinación puede ser utilizado para obtener descendencia en especies con un mayor número de cromosomas que Arabidopsis. 


\section{Acknowledgements.}

It is incredible how, after writing almost 150 pages of objective, descriptive, cold, distant and purely scientific paragraphs, these lines were to be the most difficult part; a total change in mindset. Perhaps because I knew that at the moment I had to start this last part of my thesis book, I would feel completely overwhelmed. However, even if I am struggling to find the right words and the right order to express my gratitude correctly, I will try my best.

I suppose I should start thanking the two people that initially gave me the opportunity to accomplish this: Hans de Jong and Erik Wijnker. I never thought I would find myself so comfortable and thrilled working with plants. Specially, I really need to highlight how important Erik (or so called, Boss) has been for me during my PhD, from start to end. Every now and then I still try to understand why after the interview with very poor internet connection -as I was broadcasting from somewhere in the middle of nowhere in Costa Rica-, you thought that I may do well with plants. I was your first $\mathrm{PhD}$ student and a sort of supervision-experiment, but it could not have turned out any better. You have been the most supportive and enthusiastic boss one could ever have. Thank you for trusting me with this project, and for making possible to achieve much more than what it was expected from us. You were able to transmit your passion about science like very few people can; It must have worked, though, because someone once called me mini-Erik after explaining in a presentation how reverse breeding may just very elegantly save the world. Thank you very much for being so accessible, for allowing hours and hours of endless discussions and for showing me how exciting and rewarding a future in plant sciences can be. Thank you for being so patient and, at the same time, demanding, for going through my texts over and over again until I finally learned how to -more or less- write. Thanks for helping me out to become a much better scientist. Thank you for this, and for being, besides an amazing and very humble boss, a really good friend.

And talking about friends and bosses, the next one in line could not be other than Arp. In this case I am especially grateful, because you adopted me in the lab as a sort of collateral damage, but always supported me and my work. It did not matter if I interrupted you in your office to talk about non-sense possible plant breeding outcomes, haploid inductions and viruses messing up with my tomato plants, or if I just desperately needed a coffee at the airport because I was tremendously hungover. Thank you for sharing natas in the very low moments of the PhD. I owe you a lot, and a big part of this $\mathrm{PhD}$ has been possible thanks to you, and to the great discussions during seminars (i.e. kicker). It has a been a unique period, and I will always remember the four of us having fun during COMREC pre-meetings, meetings and after-meetings, the serious talks over glasses of wine and your help from the start to the end of my $\mathrm{PhD}$, especially in those moments when a career in science gets rough and uncertain. Thanks for being there all along the process.

I think I should continue with the acknowledgments geographically, and that means, I need to thank quite a few people of the lab in Hamburg, Dave, Reinhold, Katja, Viola, Mariana, Oscar, Lucas, Franzi, Peter, Jantje, Max, Maren R. and Maren. H for all the nice discussions, lab retreats, help with the project whenever I needed it and for creating such a pleasant environment to work in.

I know that I keep saying that each of these people made possible my PhD project to happen, but actually it actually was experimentally possible mainly thanks to two of my molecular gurus, the unbeatable Asia team, Shini and Chao. Guys, you are unbelievable. Shini: you just know. I mean. You just know everything. If my experiments worked out so well, it has been undoubtfully thanks 
to the time you have spent teaching me what not to do and giving me advice. I cannot strength how much I respect you as a scientist. Thank you very much for guiding me through the darkness of the molecular work and for always showing honest interest in my work. Chao: I still cannot understand how can you keep track of so many projects. You are a natural and very talented molecular biologist and, as you always say, you should simply get your own group in China and start doing incredible things. Hopefully one day you will invite me to China for real, because you are one of the best traveling mates I have ever met and I am very much looking forward to that trip! Mami Dagmar, you are the perfect mix between fun and responsible, always looking after your children in the lab. Juanis, thanks for standing my crazy stories and impulses, the loud music in the lab, the concerts and kitesurfing courses I "invited" you to go to. Marti and Joke, I am super happy you two guys ended up in this lab, I just wish we could have shared a bit more of quality time together, but this can be solved soon! Yuki, thanks for sharing my love for matcha and art, and for always irradiating cuteness and happiness. Poyu, thank you for the always honest, smart -and most of the time funny- conversations. Kosti, you are the most stylish scientist I can always sit down and have a serious chat with, either about traveling, photography, life imaging or just life. Ika (Sis), although you are not there anymore, for me you will always be part of the Hamburg crew. Thanks a lot for taking care of me like a real big sis. Thanks for all the time you have spent with me, looking at nanodrop concentrations, ligation reactions, sick plants and gel images. Thank you for always offering me a place to stay, providing me with incredibly tasty food and for cheering up with Erik and me every time we got a positive result. Thanks for being by our side. Kleine and Felix: Thanks for all the great kicker matches, for the beers afterwards, for being such happy and easygoing fellows, and for supporting the little achievements of each other. Thank you all for this exciting, encouraging, illustrating and extremely entertaining time.

Now it comes a very sensitive part of the acknowledgments, the one dedicated to the Homies. The Homies is a group of people that through serendipity stumbled into my life becoming, unavoidably, family. The homies group is integrated by three people: La Meri, El Brother (Also known in the rest of the world as Gaetan) and Bente, my german doppelganger. Let's start with La Meri.

Ay, Meri. We have been through a lot together in these last four years! We have seen, experienced and enjoyed amazing things. We have been shocked by many others. It is just way too much to list all here (besides some of them should never be revealed). This only means that at this point, you know me very well. You realize when I am so tired that I cannot even think, travel, talk or party anymore. You make me think twice before doing something stupid, like booking 3 flights in a row or buying an ukulele to play in a Christmas party (although for this last one you were not fast enough). You knew better than anyone all the struggles, ups and downs that occurred during this time. So, I can only THANK YOU for not giving up on me when I get very stubborn, harass you to play kicker, go party, go shopping, go travelling, go hiking, go camping, go to dinner or even when I stare at a hole in a sweater (you know what I mean). Thank you for saying the right words to put me back on track when I get very lost. Thanks, La Meri, because without you, your amazing hair, your "balling" attacks and our gypsy (gipsy?) problems, no way this experience could had been the same. Now Pablo is part of it, and it only got better.

El Brother -or Gaetan- the big protector of my bubble. You, together with Eugen and Meri have opened the door of your house for me so many times during this nomad period of my PhD that I cannot even recall anymore; thanks a lot, guys. Bro, you are one of the most amazingly perfect 
human beings I have ever met, a role model. I have blind faith in you and I am extremely happy to have worked with you alongside. We have shared the perfect moments in a little French corner, when the rest of the world was just not right and this place was the only possible place to be. We have enjoyed like no one in Hamburg the quiet company of an aperol spritz. You are the most professional friend I have. Thank you for saving the day many times, professionally and personally. At this point, I can only say that I feel extremely lucky for being part of your life.

My blondie friend, Bente. You got to know me so well that you realized that when I said I was sad, it actually meant that was tired, and sent me straight to bed. We have shared so many crazy, funny, parallel, complementary stories that we could write a book with all of them. You have heard me complain about bacteria that do not grow and plants that I cannot infect as if you were involved in the experiment (poor thing). You have offered me the right amount of coffee and ibuprofen on a Saturday morning before going to the lab to work. Thank you for getting excited about my trips to conferences, talks or any other sort of scientific outcome. I am very grateful for everything you have done for me, for caring so much and for being simply best.

As I need to slowly transition towards the other side of the border, I will continue thanking another incredible flatmate I had the luck to find in Wageningen. Renake: thank you very much for basically everything you have done for me. Thank you for welcoming me in such a warm way and being always so open and kind to me. Thank you for your understanding and for helping me in a very complicated time of tiredness, frustration and detachment from reality. I really appreciate your effort in helping me finding my place in Wageningen. It was a short time but rather important and unforgettable. Oh yeah! and thank you for teaching me how to cook pao de queijo!

The transition, therefore continues geographically, moving slowly towards the genetics group in Wageningen. Here, I really need to acknowledge you all for always receiving me enthusiastically and for assisting me anytime that I displayed my broad ignorance about the matters of Wageningen University. Special thanks to Hans, once more for the support and always useful feedback during the writing of my thesis and for keeping track of all the deadlines and to do's at the end of my $\mathrm{PhD}$. To Bas, for encouraging and following up the progress of my research, for your tips and advices while writing my thesis. Frank for his extreme working efficiency and patience in explaining a third-year $\mathrm{PhD}$ student the basic regulations of the greenhouse. Thank you for understanding that trying to develop a project between two labs was not easy and for not being very annoyed with me anytime I forgot to clean my plants. Besides, it is always great to talk to you about music, beer or food. Wytske, thank you (so much, really) for taking care of all the bureaucratic issues related to my PhD. You literally have saved me a couple of times. Annelous, thanks for being always interested in my whereabouts. Ramón, mi querido paraninfo. I remembered the day I met you. You explained to me that we were in a super developed university where the coffee was for free for the employees. Then we took the bike and went to the river. During the ride, you were telling me that here, life is easy and green. And so, the years passed by. I need to thank you for all personal and professional discussions, for allowing me to stay in your place when I was coming to Wageningen (here I, of course need to thank Jordi and Carmen too). Your crazy and extravagant ideas and loyalty, made of you the best and most inspiring friend I could find in town. You are such an intelligent, creative, social, kind, passionate and dedicate scientist, that I am sure you are about to achieve the most incredible and at the same time, unexpected things (although becoming the major of Droevendaal is unfortunately not an option anymore). I am very proud of having you as my paranymph and I am very curious to see what 
impossible plan is around the corner. Cris, you, like Ramon, have helped me out in crucial moments. For instance, when my plants were growing and needed to be phenotyped but I was not in the country, or when genotyping boxes needed to be sent again for the most important experiment of the PhD. I think we have been aware of many changes in the group, and we have tried to support each other on the way. Oh! And in case I have never said it, I really admire you will power and I am still trying hard to understand how on earth you can push yourself to run for $100 \mathrm{~km}$ after a bad injury like the one you suffered from.

The fact that I became part of Wagenigen University was a lucky event, and so it was my ITN. Marina, Adri, Pablo, Divya, Meri, Gunji, Matheusz, Jason, Mikel, Sevjin, Amy and Jihed. Thanks for the most absolutely unforgettable international environment one could dream of. The outstanding presentations and discussions, the parties and the quality time together make me really nostalgic.

In The Netherlands I have met truly extraordinary people. One of them has been Lucía, my festival buddy. Lucía, tú has sido una de las personas que ha salvado el año. Conocerte en esa fiesta hizo que el viaje mereciera la pena. Muchísimas gracias por hacer un esfuerzo por mantener el contacto, por decidir pasar tiempo conmigo y por guiarme en las maravillas de mi nueva "dutch life". Contigo la diversión está asegurada, en los buenísimos momentos e incluso en los malos, cuando la razón y el corazón están en pie de guerra. Tengo que agradecerte muchísimo el apoyo que has mostrado durante estos meses, soportando mis quejidos incesantes de doctorando al final de la tesis. Gracias cuchi mía, por sacarme de casa a rastras si hace falta, por mantener conversaciones inteligentes y por ser, genuinamente, tan buena persona y amiga. Espero que hoy podamos brindar por muchos años cargados de Down The Rabbit Holes.

The other extraordinary finding was Seger, the other miracle of Down The Rabbit Hole. You could not have arrived in a better moment to my life. You are my anchor, my safety net. From day 1 you became absolutely indispensable; an essential part of me that I did not know it was missing. Gracias por tu esfuerzo por aprender español y por tu actitud positiva 24/7. Gracias por intentar involucrarte en cada aspecto de mi vida y por invertir tanta energía en nosotros. You are the one who witnessed the struggles and little successes that occurred during the most frightening part of my thesis. I have to be incredibly thankful to you; not only you have supported me to continue doing what I was supposed to do, but you also pushed me to become the best me I could possibly be. Thanks to you, I did not doubt that finishing this thesis was possible. You encouraged me to continue working, day after day. You took care of me when I was not able to take care of myself, making me realize that this was just a step, and that another step would just come afterwards. This is how it goes, and I am just looking forward to the next steps in our life together, whether these are taken in Amsterdam or anywhere else in the world.

Y puestos a llevar a cabo cambios fronterizos, dejo lo más intenso para el final, cuando ya soy libre de escribir lo que quiera escribir, de manera que las palabras no se atraviesen y los significados estén perfectamente calculados. En este apartado no puedo olvidarme de dar las gracias a mis queridas Kachimberas: Liu, Ni, María, Miru, Amaya, Be, Ana, Saray y Cris. Si me paro a pensarlo, es increíble que esté incluyendo en los agradecimientos de mi tesis a personas que han estado en mi vida durante tanto tiempo. Cada vez que nos juntamos, tocamos madera para que esto nunca acabe. Gracias chicas por intentar entender mis experimentos, por apoyar esta tesis y por recibirme con los brazos abiertos cada vez que vuelvo a casa, como si nada 
hubiera cambiado. Y lo bonito es que, en realidad, nada ha cambiado para nosotras. Cada año que pasamos lejos, me doy cuenta de que siempre estamos muy cerca. Gracias a todas por ser mi hogar más antiguo y más seguro.

Y ya por último, por supuesto, no puedo dejar de agradecer de la manera mas sincera y clara posible, a mi familia. Gracias papá y mamá por apoyar a vuestra hija Willy Fog. Yo sé que no es fácil seguirme la pista, que no paro de moverme, que paso la mitad de mi vida entre estaciones, pero gracias por preocuparos en cada momento de cómo estoy, por hacer posible la carrera de la segunda científica en la familia y por entender que no queda otro remedio que estar fuera de casa. Este año ha sido más evidente que nunca que tener a un miembro de la familia lejos cuesta, pero aún queda mucho por vivir y por disfrutar juntos. Que hoy estéis aquí hace realmente pueda estar orgullosa de mi misma, pero, sobre todo, infinitamente agradecida porque si esto ha sido posible, ha sido gracias a vosotros. Y por eso, os seguiré queriendo tanto, aquí (o como dirías tú, mamá) o en Pekín. Aunque tengo que admitir, que el que realmente puso la semilla de la ciencia en mi cabecita fuiste tú, Fer. Recuerdo como si fuera ayer el día que me perseguías por el pasillo de casa repitiendo una y otra vez la definición de la clorofila, hasta el punto que aún soy capaz de repetirla después de... ¿16 años? También recuerdo como, inevitablemente, quería seguir los pasos de mi hermano mayor, el neurocientífico. Al final elegí un camino más verde, pero que sé que ha estado condicionado en gran medida por ti, por ser un ejemplo espectacular de trabajo duro, perseverancia y calidad científica. Gracias por cuidar de mi desde la distancia y por los maravillosos viajes por el mundo que hemos hecho (sigo pensando que el mejor viaje de mi vida ha sido Hawaii). Gracias por sacrificarte por la familia cuando hace falta y por ponerte detrás del volante si necesitamos tu presencia. Aunque en ese sentido, no puedo dejar de agradecerte a ti también Roci, que no te cueste montar a esos tres pequeños preciosos milagros en el coche para venir a vernos. Sé que nos es fácil mantener el dónde y el cómo de cada uno de nosotros, pero de verdad agradezco tu esfuerzo por hacer que al final todos podamos pasar tiempo con los que de verdad nos importan. Os quiero mucho, hermanitos y, esperando que un día lean esto, tened muy por seguro que os quiero mucho también, Daniela, Hector y Emma.

Igualmente, tengo que agradecer a todos mis primos y tíos, a los que cada vez estoy mas unida, por su preocupación sincera y por su interés en mis idas y venidas. Ojalá os pudiera dedicar un párrafo eterno a cada uno, pero me estoy quedando sin papel y sin palabras para agradecer.

Aún me quedan personas en el tintero: Laure, Cami, Ro, La Panda (especialmente mi querida bolilla de amor, Gusi, por darme cobijo en Madrid tras cada escapada a casa), Barby, Joan y Elena. Also, to my dutch family, Alie, Joke, Ewoud, Bernadette, Volk, Saskia, and of course little cutie Imme, as they have always welcomed me as one more in the family. For this I am immensely grateful and I hope we can keep celebrating the good news in this family for many years.

If I forgot someone, I am very sorry but I hope most of you feel how important your presence has been in the last four years of my life.

And with this I am done. Wow. The last words I write in my PhD thesis.

Well, it has been an exciting -and no doubt- unique trip. 


\section{Curriculum vitae.}

Vanesa Calvo-Baltanás was borned in Palencia, a little province of Spain, on the 22 ${ }^{\text {nd }}$ of August, 1990. She attended the Teresianas Primary School and the Instituto de Educación Secundaria Recesvinto in Dueñas. She finalized her secondary studies in the instituto Alonso Berruguete in Palencia. Biology was always her favorite subject and following the steps of her older brother, she felt encouraged to also pursue a career in research.

She started her studies in biology and life sciences in 2008 at the University of Salamanca, in Spain. After the first three years of bachelor, in 2011 she obtained an Erasmus grant that allowed her to live in Iceland for one year. There, she could work in two different research projects in the lab of Arnar Pálsson, at Háskóli Íslands University. Both projects were part of a bigger effort to characterize the four different sub-species of the endemic Arctic charr (Salvelinus alpinus) that cohabited in the lake Thingvallavatn. In these projects, she was assessing differences in phenotypes between two different icelandic endemic trout populations, evaluating traits such as life-span, body length and morphological differences during embryo development. Another part of the project required the genetic characterization of the MHC2alfa locus. This locus encodes for a class of molecules that play a major role during the immune response, specifically in antigen recognition and antigen presentation. As a main outcome of these projects, she concluded that the differences in allelic frequencies found between the different sub-populations of Artic charr for the MHC2alfa locus, directly correlated with the survival rate of the individuals in the population. The time invested in these two projects was sufficient to trigger her passion for molecular biology and molecular genetics. After the two research projects developed in Háskóli Íslands, she started to work at the research facilities of Stofnfiskur, a company for selective breeding and egg production of salmon in Iceland. Supervised by Eduardo Rodriguez, she was working in a project that required high-throughput genotyping and marker-assisted selection.

She graduated in 2013 by the University of Salamanca, with a specialization in life sciences and biotechnology. In 2014 she went to the Pacuare Nature Reserve in Costa Rica to be hired as field researcher in a conservation programme to protect the endangered sea turtle species Dermochelys coriacea. After this period, she realized that, although she loved this kind rewarding job, her place was in the lab.

At the end of the summer of 2014 she was recruited to be part of the COMREC Marie Curie ITN. Under the supervision of Erik Wijnker, Arp Schnittger and Hans de Jong she started her PhD project in molecular plant breeding and plant meiosis in Wageningen University and Research in The Netherlands and Hamburg University, In Germany. The aim of her PhD was to efficiently downregulate meiotic genes in the model organism Arabidopsis thaliana using a transient silencing technique called virus-induced gene silencing (VIGS). The establishment of this technique served to effectively develop breeding methods, such as the application of reverse breeding in wild-type plants or the generation of polyploid plants with a specific genetic make-up. She now continues with her nomad lifestyle, carrying out her research between Wageningen and Hamburg, and living a happy life, whenever possible, in Amsterdam with her boyfriend, Seger. 


\section{Publications.}

Kalina H. Kapralova, Johannes Gudbrandsson, Sigrun Reynisdottir, Cristina B. Santos, Vanessa C. Baltanás, Valerie H. Maier, Sigurdur S. Snorrason, Arnar Palsson. 2013. Differentiation at the MHClla and Cath2 Loci in Sympatric Salvelinus alpinus Resource Morphs in Lake Thingvallavatn. Plos One. https://doi.org/10.1371/journal.pone.0069402.

Vanesa Calvo-Baltanas, Cris L. Wijnen, Nina Lukhovitskaya, C. Bastiaan de Snoo, Linus Hohenwarter, Hans de Jong, Arp Schnittger, Erik Wijnker. Efficient reverse breeding by VIGSmediated crossover reduction. Biorxiv. https://doi.org/10.1101/459016 
Education Statement of the Graduate School Experimental Plant Sciences

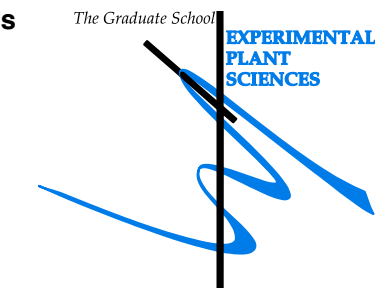

Issued to: $\quad$ Vanesa Calvo Baltanas

Date: $\quad 15$ February 2019

Group: Laboratory of Genetics

University: Wageningen University \& Research

\begin{tabular}{|c|c|c|c|}
\hline \multicolumn{2}{|c|}{ 1) Start-Up Phase } & \multirow[t]{2}{*}{$\underline{\text { date }}$} & \multirow[t]{2}{*}{$\underline{c p}$} \\
\hline$\checkmark$ & First presentation of your project & & \\
\hline & Project summary and preliminary results & 25 Oct 2014 & 1,5 \\
\hline 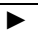 & Writing or rewriting a project proposal & & \\
\hline$\nabla$ & Writing a review or book chapter & & \\
\hline$\nabla$ & MSc courses & & \\
\hline \multicolumn{2}{|c|}{ Subtotal Start-Up Phase } & & 1,5 \\
\hline \multicolumn{2}{|c|}{ 2) Scientific Exposure } & $\underline{\text { date }}$ & $\underline{c p}$ \\
\hline \multirow[t]{3}{*}{$\checkmark$} & EPS PhD student days & & \\
\hline & PhD Student day 2016, Soest, The Netherlands & 28-29 Jan 2016 & 0,6 \\
\hline & PhD Student day 2017, Soest, The Netherlands & 09-10 Feb 2017 & 0,6 \\
\hline \multirow[t]{3}{*}{$\nabla$} & EPS theme symposia & & \\
\hline & EPS theme 4 'Genome Biology', Amsterdam, The Netherlands & 15 Dec 2015 & 0,3 \\
\hline & EPS theme 4 'Genome Biology' Amsterdam, The Netherlands & 25 Sep 2018 & 0,3 \\
\hline \multirow[t]{2}{*}{$\triangleright$} & $\begin{array}{l}\text { National meetings (e.g. Lunteren days) and other National } \\
\text { Platforms }\end{array}$ & & \\
\hline & Annual meeting 'Experimental Plant Sciences', Lunteren & 11-12 Apr 2016 & 0,6 \\
\hline \multirow[t]{8}{*}{$\triangleright$} & Seminars (series), workshops and symposia & & \\
\hline & $\begin{array}{l}\text { 1. Workshop: control of meiotic recombination/plant breeding, } \\
\text { Birmingham, UK }\end{array}$ & 9 July 2014 & 0,3 \\
\hline & 2. Workshop: Bioinformatics. Wageningen, The Netherlands & 4-6 Feb 2015 & 0,9 \\
\hline & $\begin{array}{l}\text { 3. Workshop: advanced methodologies in meiosis research, El Escorial, } \\
\text { Madrid, Spain }\end{array}$ & 5 May 2015 & 0,3 \\
\hline & 4. Workshop: methods and scienfic writing, El Escorial, Madrid, Spain & 6 May 2015 & 0,2 \\
\hline & 5. Workshop on commercial plant breeding, Gatersleben, Germany. & 18 May 2016 & 0,2 \\
\hline & 6. Workshop:Research dissemination Cambridge UK & 11 May 2017 & 0,3 \\
\hline & Seminar: "Meiosis" Gordon Conference, New London, EEUU & 25-26 Jun 2016 & 0,6 \\
\hline$\nabla$ & Seminar plus & & \\
\hline \multirow[t]{4}{*}{$\triangleright$} & International symposia and congresses & & \\
\hline & 1st COMREC annual meeting, El Escorial, Madrid, Spain & 4 May 2015 & 0,3 \\
\hline & 2nd COMREC annual meeting, IPK, Gatersleben, Germany & 17 May 2016 & 0,3 \\
\hline & Final COMREC meeting, Cambridge, UK & 10 May 2017 & 0,3 \\
\hline
\end{tabular}




\begin{tabular}{|c|c|c|c|}
\hline & Conference: Gordon "Meiosis", New London, EEUU & $\begin{array}{l}26 \text { Jun }-1 \text { Jul } \\
2016\end{array}$ & 1,8 \\
\hline & Conference: EMBO "Meiosis", Oxford, UK & $\begin{array}{l}30 \text { Aug - } 4 \text { Sep } \\
2015\end{array}$ & 1,5 \\
\hline & Conference PAG San Diego, US & 14-18 Jan, 2017 & 1,5 \\
\hline & Conference EMBO "meiosis", Hvar, Croatia & $\begin{array}{l}27 \text { Aug - } 1 \text { Sep } \\
2017\end{array}$ & 1,5 \\
\hline & Conference PAG, San Diego, US & $13-17$ Jan 2018 & 1,5 \\
\hline \multirow[t]{11}{*}{$\square$} & Presentations & & \\
\hline & Talk: COMREC 1st annual & 4 May 2015 & 1,0 \\
\hline & Talk: EPS Theme 4 & 15 Dec 2015 & 1,0 \\
\hline & Talk: Food Security, & 6 Apr 2016 & 1,0 \\
\hline & Talk: COMREC 2nd annual meeting, IPK, Gatersleben, Germany & 17 May 2016 & 1,0 \\
\hline & Talk, COMREC final meeting & 16 May 2017 & 1,0 \\
\hline & Talk: PAG Analysis of complex genomes. San Diego, USA & 13 Jan 2018 & 1,0 \\
\hline & Talk: PAG Components of apomixis. San Diego, USA & 16 Jan 2018 & 1,0 \\
\hline & Poster: Development of cytogenetic tools for advancing breeding. EMBO & $\begin{array}{l}30 \text { Aug - } 4 \text { Sep } \\
2015\end{array}$ & 1,0 \\
\hline & Poster: Hybrid breeding through simplified meiosis. Gordon & $\begin{array}{l}26 \text { Jun }-1 \text { Jul } \\
2016\end{array}$ & 1,0 \\
\hline & $\begin{array}{l}\text { Poster: Business, breeding and science "Green talents" Hamburg, } \\
\text { Germany }\end{array}$ & 20 Oct 2016 & 1,0 \\
\hline$\checkmark$ & IAB interview & & \\
\hline$\checkmark$ & Excursions & & \\
\hline \multicolumn{2}{|c|}{ Subtotal Scientific Exposure } & & 23,9 \\
\hline \multicolumn{2}{|c|}{ 3) In-Depth Studies } & $\underline{\text { date }}$ & $\underline{c p}$ \\
\hline \multirow[t]{10}{*}{ 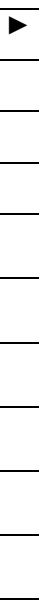 } & EPS courses or other PhD courses & & \\
\hline & Current progress in meiotic research 1, Helgoland, Germany & $28-30$ Sep 2015 & 0,6 \\
\hline & Current progress in meiotic research 2, Sylt, Germany & 26- 29 Sep 2016 & 0,6 \\
\hline & Current progress in meiotic research 3, Hiddensee, Germany & 25- 28 Sep 2017 & 0,6 \\
\hline & Current topics in developmental biology, Hamburg Germany & $\begin{array}{l}\text { Winter Term } \\
2014 / 2015\end{array}$ & 0,3 \\
\hline & Current topics in developmental biology, Hamburg Germany & $\begin{array}{l}\text { Summer Term } \\
2015\end{array}$ & 0,3 \\
\hline & Current topics in developmental biology, Hamburg Germany & $\begin{array}{l}\text { Winter Term } \\
2015 / 2016\end{array}$ & 0,3 \\
\hline & Current topics in developmental biology, Hamburg Germany & $\begin{array}{l}\text { Summer Term } \\
2016\end{array}$ & 0,3 \\
\hline & Current topics in developmental biology, Hamburg Germany & $\begin{array}{l}\text { Winter Term } \\
2016 / 2017 \\
\end{array}$ & 0,3 \\
\hline & Current topics in developmental biology, Hamburg Germany & $\begin{array}{l}\text { Summer Term } \\
2017\end{array}$ & 0,3 \\
\hline \multirow[t]{2}{*}{ 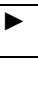 } & Journal club & & \\
\hline & Attendance journal club & $2014-2018$ & 3,0 \\
\hline$\triangleright$ & Individual research training & & \\
\hline
\end{tabular}




\begin{tabular}{|c|c|c|c|}
\hline \multicolumn{2}{|c|}{ Subtotal In-Depth Studies } & & 6,6 \\
\hline \multicolumn{2}{|c|}{ 4) Personal Development } & $\underline{\text { date }}$ & $\underline{c p}$ \\
\hline \multirow[t]{9}{*}{$\checkmark$} & Skill training courses & & \\
\hline & EPS introduction course, WUR, Wageningen, The Netherlands & 12 Feb 2016 & 0,3 \\
\hline & $\begin{array}{l}\text { Workshop on busienss skills and entrepreneurship, Gatersleben, } \\
\text { Germany }\end{array}$ & 18 May 2016 & 0,2 \\
\hline & Scientific publishing WUR, Wageningen, The Netherlands & 13 Oct 2016 & 0,3 \\
\hline & Brain training WUR, Wageningen, The Netherlands & 20 Jun 2018 & 0,3 \\
\hline & Career assessment,WUR, Wageningen, The Netherlands & Aug 2018 & 0,3 \\
\hline & Posters and pitching, WUR, Wageningen, The Netherlands & May-Jun 2018 & 1,0 \\
\hline & Writing grant proposals, WUR, Wageningen, The Netherlands & Sep-Nov 2018 & 2,0 \\
\hline & BCF Career Event & 24 May 2018 & 0,3 \\
\hline \multirow[t]{3}{*}{$\triangleright$} & Organisation of PhD students day, course or conference & & \\
\hline & $\begin{array}{l}\text { Outreach: Nacht das Wissens (science for the public) Hamburg, } \\
\text { Germany }\end{array}$ & 7 Nov 2015 & 1,5 \\
\hline & Outreach event -Thinktank. Birmingham, UK. & 19 Jan 2016 & 1,5 \\
\hline$\nabla$ & Membership of Board, Committee or PhD council & & \\
\hline \multicolumn{2}{|c|}{ Subtotal Personal Development } & & 7,7 \\
\hline \multicolumn{2}{|c|}{ TOTAL NUMBER OF CREDIT POINTS* } & & 39,7 \\
\hline \multicolumn{4}{|c|}{$\begin{array}{l}\text { Herewith the Graduate School declares that the PhD candidate has complied with the educational requirements } \\
\text { set by the Educational Committee of EPS which comprises of a minimum total of } 30 \text { ECTS credits. }\end{array}$} \\
\hline & dit represents a normative study load of 28 hours of study. & & \\
\hline
\end{tabular}





\section{COLOFON.}

The research in this thesis was carried out in the Laboratory of Genetics at Wageningen University and Research, Wageningen, The Netherlands, in collaboration with the department of Developmental Biology in Hamburg University, Germany and was financially supported by the People Programme (Marie Curie Actions) of the European Union's Seventh Framework Programme FP7/2007-2013/ under REA grant agreement $\mathrm{n}^{\circ}[606956] 13$.

Cover design by: Vanesa Calvo-Baltanás

Thesis layout by: Vanesa Calvo-Baltanás

Printed by: GVO drukkers \& vormgevers B.V 